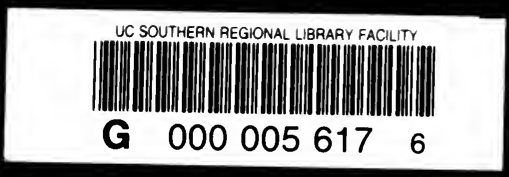




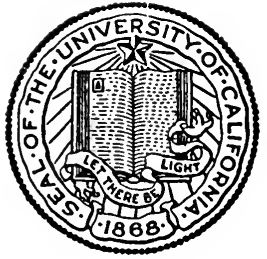

THE LIBRARY OF THE UNIVERSITY OF CALIFORNIA LOS ANGELES

YIIIT 
newl 


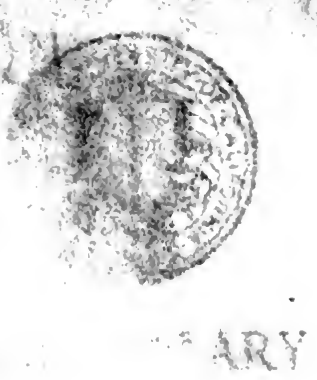

Tons?

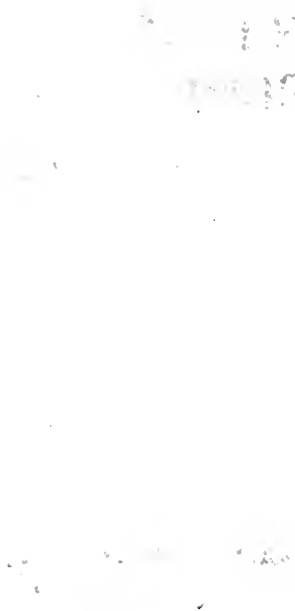

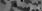

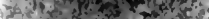

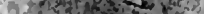

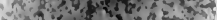

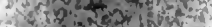

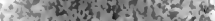

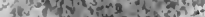

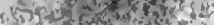

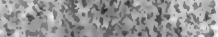

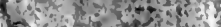

,

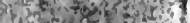
monas.

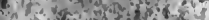

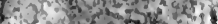

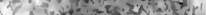

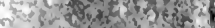
ynd

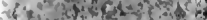

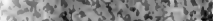

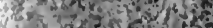

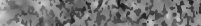

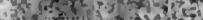
a c Shens yerand

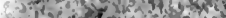
an in

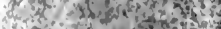

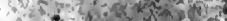
$4=0$ -

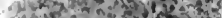

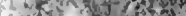

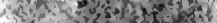

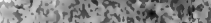

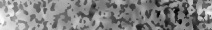

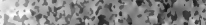

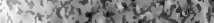
Heles

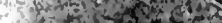

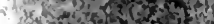

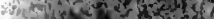
wroving $2 \frac{1}{2} x^{2} y$ 


\title{
ATLAS AND EPITOME
}

OF

\section{OPHTHALMOSCOPY}

AND

\section{Ophthalmoscopic Diagnosis}

\author{
BY \\ PROF. DR. O. HAAB \\ of Zurich
}

SECOND AMERICAN EDITION FROM THE FIFTH REVISED AND ENLARGED GERMAN EDITION

EDITED BY

G. E. DE SCHWEINITZ, A.M., M.D.

Professor of Ophthalmology in the University of Pennsylvania and Ophthalmic Surgeon to the University Hospital ; Consulting Ophthalmic Surgeon to the Philadelphia Polyclinic; Ophthalmic Surgeon to the Philadelphia General Hospital ; Ophthalmologist to the Orthopedic Hospital and Infirmary for Nervous Diseases

With 152 Colored Lithographic Illustrations

PHILADELPHIA AND LONDON

W. B. SAUNDERS COMPANY 
Set up, Electrotyped, Printed, and Copyrighted May, 1901, Reprinted September, 1904, March, 1905, and March, 1906. Revised, Reprinted, and Recopyrighted March, 1909

\section{Copyright, 1909, by W. B. SAUNDERS COMPANY}

- Reprinted March, I9ro

PRINTED IN AMERICA

PRESS OF

W. B. SAUNDERS COMPANY

PHILADELPHIA 


\section{EDITOR'S PREFACE TO SECOND EDITION}

THE continued favor which has been accorded to Professor Haab's Ophthalmoscopy and Ophthalmic Diagnosis has made necessary a new edition, in which the subjectmatter has been brought up to date and a few new chromo-lithographs have been added, several from Professor Haab's collection and one from the collection of the editor. It can scarcely be doubted that this book will prove in the future, as it has in the past, of the greatest use to those who desire to study and teach Ophthalmology.

\section{EDITOR'S PREFACE TO FIRST EDITION}

THE great value of Professor Haab's Atlas of Ophthalmoscopy and Ophthalmoscopic Diagnosis has been fully established, and entirely justifies an English translation of his latest edition. Not only is the student made acquainted with carefully prepared ophthalmoscopic drawings, done into well-executed lithographs, of the most important fundus-changes, but in many instances plates of the microscopic lesions are added. The whole fur- nishes a manual of the greatest possible service, not only to the beginner in ophthalmic work, but to one who has already far advanced and desires to compare the observa- 
tions of his own service with those of the rich clinic from which Professor Haab has gathered his plaies. A few figures have been added by the editor-namely, those showing Angioid Streaks in the Retina, and the Ophthalmoscopic Appearances seen in Arteriosclerosis. As in the Atlas of External Diseases of the Eye, produced under the same editorship, occasional comments are placed in brackets. It is sincerely trusted that this book will prove of great use to those who desire to study and teach Ophthalmology.

\section{PREFACE TO FIFTH EDITION}

Although this atlas was already very much greater than the first edition, it was nevertheless possible, thanks to the kindness of the publisher, to enlarge the present fifth edition by the addition of two illustrations of birth injuries of the eve. An illustration of pigmentary degeneration of the retina which was not quite satisfactory was replaced by a better one. In addition, several of the earlier illustrations were retouched and corrected in various ways. Some corrections and additions have also been made in the text, and it is hoped that this edition, like its predecessors, may meet with the approval of the profession.
O. HAAB. 


\section{PREFACE TO THE SECOND EDITION.}

THE kind reception accorded to this volume in various countries induced me to make a number of additions and corrections before the book went through the second edition. Besides adding to the text, I enriched the illustrated portion of the work by a number of anatomic figures to illustrate the differences between normal and pathologic appearances of the eye-ground. They are intended to explain the things seen under the microscope and their topographic relations, thus enriching the student's pathologic knowledge and enabling him to interpret more accurately the clinical pictures presented by the various diseases.

A few of the original ophthalmoscopic pictures have been replaced by better ones, and two have been added that are entirely new (retinitis circinata and true staphyloma in myopia).

For the preparation of the anatomic figures I am indebted to the skill of our academic artist, Mr. L. Schröter.

Although the pupillometer (Fig. 80,a) does not, strictly speaking, belong to the subject of ophthalmoscopy, I have, nevertheless, yielding to a long-cherished desire, incorporated it in this book-which is, above all, intended for practical, every-day use-believing that many 
practitioners who feel the necessity of determining the size of the pupil more accurately than is possible by mere inspection will find it a welcome addition to their clinical armamentarium.

Finally, I wish to express my grateful recognition of the great care and effort bestowed on the preparation of this Atlas by the publisher.

O. HAAB. 


\section{CONTENTS.}

INTRODUCTION . . . . . . . . . . . . . . . . . . . . 13

Description of the Ophthalmoscope . . . . . . . . . 18

Examination in the Erect Image . . . . . . . . . 23

Measurement of the Myopic Eye . . . . . . . . 26

Measurement of the Hypermetropic Eye . . . . . . . . 29

Measurement of Astigmatism . . . . . . . . . . 34

Size of the Ophthalmoscopic Field of Vision . . . . . 38

Examination By the Indirect IIEThod . . . . . . 40

Size of the Visual Field . . . . . . . . . . . . 42

Enlargement of the Image in the Direct and in the Indirect Methods... . . . . . . . . . . 43

Measurement of Refraction by the Indirect Method . . 45

Determination of Irregularities in the Surface of the Eyeground ... . . . . . . . . . . 45

Examination by Transmitted Light . . . . . . 47

ShadOW-TEST, oR SKIASCOPY . . . . . . . . . . . 53

Choice of an Ophthalmoscope . . . . . . . . . . . 58

Method of Conducting ax Ophthalmoscopic Examination 67

Normal Eye-Ground . . . . . . . . . . . . . 74

Pulsation Phenomena . . . . . . . . . . . . . . 82

INDEX . . . . . . . . . . . 89 



\section{ILLUSTRATI ONS.}

FIG.

1. Normal Eye-ground.

2, $a, b$. Section of a Normal Papilla.

3. Section of the Retina, Choroid, and Contiguous Sclera in a Normal Eve.

4. Normal Eye-ground (Blonde).

$5, a, b$. Normal Eye-ground (Dark).

6. Medullated Nerve-fibers in the Retina.

7. Congenital Circumscribed Defect of the Choroid.

8, a. Congenital Circumscribed Defect of the Choroid and Malformation of the Optic Nerve.

8, b. Congenital Defect of the Pigment-epithelium of the Retina.

9, a. Congenital Circumscribed Defect of the Retinal Pigment and the Choroid.

9, b. Congenital Circumscribed Defect of the Choroid.

10, a. Congenital Cireumscribed Defect of the Choroid.

10, b. Congenital Absence of Pigment.

11. Congenital Dislocation of the Lens.

$12, a, b$. Inflammation of the Optic Nerve.

13, $a, b$. Inflammation of the Optic Nerve in Brain-tumor.

13, A. Optic Neuritis (Choked Disc) and Macular Changes in Braintumor.

14, a-c. Horizontal Section of a Normal Macula Lutea.

15, a. Section through the Papilla in Neuritis.

15, b. Section through the Papilla in Neuritis and Papillitis.

16. Inflammation of the Optic Nerve and Retina in Syphilis.

17. Intense Inflammation of the Optic Nerve.

18. Inflammation and Congestion of the Optic Nerve in Orbital Tumor.

19, $a, b$. Atrophy of the Optic Nerve.

20, $a, b$. Gray Atrophy of the Optic Nerve.

21, $a$. Section through Entrance of the Optic Nerve in Partial Atrophy.

21, b. Section through the Disk in Total Atrophy of the Optic Nerve. 22. Atrophy of the Optic Nerve from Glaucoma.

23, $a, b$. Glaucomatous Excavation of the Optic Nerve.

24, a. Section through the Region of the Angle of the Anterior Chamber. 
FIG.

24, b. Section through Same Region, showing Obliteration of Angle.

$24, c, d$. Section through the Head of the Optic Nerve in Glaucoma.

25, $a, b$. Retina and Optic Nerve in Albuminuria.

26, a. Section through the Retina in Retinitis Albuminurica.

$26, b, c$. Varicose Nerve-fibers.

27. Retina in Retinitis Albuminurica.

28, a. Albuminuric Changes in Retina and Optic Nerve.

28, b. Albuminuric Disease of the Eye-ground.

29, $a, b$. Albuminuric Retinitis of Both Eyes.

$30, a, b$. Eye-ground in Diabetes.

31. Eye-ground in Pernicious Anemia.

32. Obstruction of the Central Artery.

$33, a$. Thrombosis of the Superior Temporal Vein.

33, b. Thrombosis of the Central Vein of the Retina.

34. Obstruction of the Superior Temporal Artery of the Retina.

35. Recurring Hemorrhages in the Retina and Vitreous.

36. Syphilitic Disease of the Retinal Arteries.

37. Syphilitic Neuroretinitis and Disease of the Retinal Arteries.

37, a. Angioid Streaks in the Retina.

$37, b$. Changes in the Eye-ground in Arteriosclerosis.

38, 39. Pigmentary Degeneration of the Retina.

40. Eye-ground in Hereditary Syphilis.

41, 42. Eye-ground in Congenital Syphilis.

43. Secondary Pigmentation of the Retina in Disseminated Choroiditis.

44, 45. Disease of the Macula Lutea due to Myopia.

46, $a$. Secondary Pigmentation of the Retina.

46, b. Sagittal Section of an Eye with a Total or Funnel-shaped

Retinal Detachment.

46, c, d. Pigmentary Degeneration of the Retina.

47. Disease of the Macula Lutea due to Old Age.

48. Disease of the Macula Lutea (Traumatic).

49. Perforation of the Macula Lutea after Contusion of Eyeball.

50, a. Macula Lutea and Surroundings in Disease from Orbital Tumor.

$50, b, c$. Retina in Thrombosis of the Vena Centralis Retinæ.

51,52 . Opacity of the Retina.

53. Disease of the Macula Lutea due to Foreign Body.

54. Disease of the Macula Lutea due to Pressure and Contusion.

55. Injury of the Retina by an Iron Splinter.

56. Air-bubble in Vitreous.

57. Old Injury of the Retina by an Iron Splinter. 
FIG.

58, a. Point of Impact of a Foreign Body on the Eye-ground.

$58, b$. Retinal Bands due to Traumatism.

58, I, a. Changes in Eye-ground Resulting from Severe Forceps Delivery. 58, I, b. Atrophy of the Optic Nerve and Retinal Vessels; Macular

Disease; Strand of Connective Tissue in Vitreous.

59. Retinal Detachment in the Temporal Portion of the Eyeground.

60. Retinal Bands and Detachment after a Punctured Wound.

61. Retinal Detachment at the Inner Upper and Lower Portions.

62. Retinal Detachment with Laceration.

63. Hemorrhagic Retinitis of Pregnancy.

64. Retinitis Circinata.

65. Eye-ground in Leukemia.

66. Glioma of the Retina.

67, a. Retina in Pernicious Anemia.

67, b. Small Inflammatory Focus in Disseminated Choroiditis.

68, 69. Disseminated Choroiditis.

70. Infiltrations of the Choroid in Disseminated Choroiditis.

71. Disseminated Choroiditis in the Early Stage.

72, a. Hyaline Bodies (Drusen) in the Vitreous Layer of the Choroid.

72, b. Senile Pigmentation of the Retina.

73, a. Recent Disseminated Inflammation of the Choroid, Overlying Retina, and Optic Nerve.

73, b. Disseminated Choroiditis,

74. Chronic Disseminated Choroiditis and Secondary Pigmentation of the Retina.

75. Miliary Tubercles in the Choroid.

76. Chronic Tuberculosis of the Choroid.

77, 78. Sarcoma of the Choroid.

79. Recent Disseminated Choroiditis.

80. Changes in the Choroid due to Contusions; Lacerations.

81. Sclerosis of the Choroidal Vessels; Disseminated Choroiditis and Secondary Pigmentation of the Retina.

82, a. Hyaline Bodies (Drusen) of the Vitreous Layer.

82, $b$. Miliary Tubercle of the Choroid.

82, c. Large Tubercular Growth in the Choroid.

83. Choroidal Sarcoma.

84. Atrophy of Choroid in Myopia.

85. True Staphyloma.

86. Posterior Venæ Vorticosæ.

87. Pupillometer. 



\section{OPHTHALMOSCOPY.}

\section{INTRODUCTION.}

Examination of the eye-ground by means of the ophthalmoscope is of the highest importance for the recognition not only of many affections of the eye itself, but also of a great number of diseases chiefly affecting organs outside of the eye, as the brain, the kidneys, or the circulatory system, and endangering life either by disturbing the general nutrition or by setting up a general infection. The wide lymph-spaces of the eye and the rich network of vessels in the retina offer a favorable soil for the development of many pathogenic germs and toxic substances present in the body. This is especially noteworthy in syphilis, both in the hereditary and in the acquired variety, although the effects of tubercular and rheumatic infection of the organism also not infrequently manifest themselves in the eye.

For these reasons ophthalmoscopic examination of the eye-ground is one of the most important methods of medical examination. Unfortunately, it is also one of the most difficult. This is partly because the familiarity with the technic of the ophthalmoscope necessary for obtaining a clear image of the eye-ground requires a certain amount of training and practice, and partly because the correct interpretation of the ophthalmoscopic image is often a very difficult matter. An astonishing variety of pictures may be seen in the interior of the eye. The image of the eye-ground varies greatly even in conditions of health, and it is anything but easy for the beginner in the art of ophthalmoscopy to determine, 
in many instances, whether the conditions present are normal or pathologic. If, for instance, he should describe the fundus of the eye as "abnormally reddened," an expert would know that he has to deal with a beginner.

Still more difficult is the interpretation of the numerous deviations from the normal which may be seen in the eye-ground. It is comparatively easy for one who sees only the coarser variations, because he fails to utilize the instrument to its fullest extent, or because the instrument itself is imperfect, or, possibly, because his eye is not sufficiently well-trained or is defective, or his examination is too hasty. Even the expert often finds the greatest difficulty in seeing and correctly interpreting the more minute pathologic alterations in the eye-ground. Practice and experience, both the examiner's own and that of other observers, in this, as in many other cases, will prove to be the best guides. The latter may be either described in words or illustrated by pictures which more or less faithfully reproduce pathologic alterations.

Mere verbal descriptions are even more unsatisfactory than they are in other similarly complicated domains of medicine, especially if the student is imperfectly acquainted with the subject. Even topographical drawings of pathologic alterations in the eye are extremely complicated, and a correct description of the coloring is often extremely difficult or even impossible. Thus, a little more white, or a little more red or gray, may make an important difference in the appearance of the optic nerve, and may be enough to show the expert that he has to deal with a serious condition, though to the inexperienced eye the appearance may be normal. It is for this reason that the examination of the eye-ground by means of the ophthalmoscope offers an excellent means of training the eye for the perception of the finest shades of color-a most useful faculty for enabling one to recognize many other morbid alterations in the body. Black and white pictures of the eye-ground have, therefore, very little 
value. They may reproduce the drawing and topography, but as they fail to give the important element of color, they can be understood and utilized only by one who already possesses a fair knowledge of the conditions portrayed. ${ }^{1}$

To supplement one's own observations and profit by those of others, one must use carefully colored illustrations.

Although we already possess a great number of illustrations of the eye-ground, both in the usual atlases and in other works, $I$ am none the less determined to publish this epitome and atlas of ophthalmoscopy. In the first place, the pictures that exist, especially those that are scattered through the literature, are not accessible to everyone, and I find that many a picture which would be useful to the student, to the practising physician, or to the clinical teacher for the purpose of study and demonstration, is not found in these works. Too many of the pictures reproduce rare conditions which even a man of large practice might not see more than once or twice in his experience. Again, many pictures of immense practical importance, especially such as illustrate subvarieties and different stages of the sarne morbid process, are often left out. Thus, for instance, it is impossible to explain to the beginner the variations of the retina and optic nerve in albuminuria by means of one or two pictures, or the manifold forms of chronic choroiditis with three or four illustrations. Accordingly, I have tried to avoid showing very rare conditions, and have instead collected as many pictures of practical importance as possible. All the ophthalmoscopic images contained in this volume were drawn from life by myself in the course of my practice, and the original ophthalmoscopic pictures are also of my own preparation. In doing this work the sketch-book ${ }^{2}$ was found extremely useful,

I It is very much to be regretted that the illustrations in the excellent work by Gowers, Medical Ophthalmoscopy, are not all colored.

2 Sketch-book for Ophthalmoscopic Observations of the Eye-ground, second edition, unbound. Published by J. F. Lehman, in Munich, 1898. 
because it combines rapid execution with the greatest possible degree of accuracy in reproducing the various shades of color. With the exception of three (Figs. 10, $b$, 39 , and $45, a$ ), all the original pictures in this book have been prepared according to my own method, and I am convinced that it is the easiest, and what is more important in drawing from life, the quickest method of obtaining a picture of all the alterations seen in the eye-ground.

To become an expert in the beautiful art of ophthalmoscopy it is necessary to do a great deal of drawing from life. I have become more and more convinced of this during the preparation of the pictures in the present volume. The eye-ground would be studied with much more care if the student were to draw ${ }^{1}$ what he sees, and especially if he were also to reproduce the colors. The benefit derived from a course in ophthalmoscopy will be very much greater if the student draws what he sees. I constantly regret that our medical students are, as a rule, so badly instructed in the art of drawing, and that so few of them can be expected to produce a fairly decent picture; but even an imperfect picture is better than none at all for beginners.

It is particularly desirable for the student and practising physician to have his ophthalmoscopic pictures in a more convenient form than the usual atlases, and the idea of presenting them in book form seems to me an excellent one.

The pictures in this volume are represented as they appear in the inverted image ; that is to say, with moderate magnification, although I, of course, also used the more highly magnified image obtained by the direct method in preparing the sketches which are taken from life. The figures, therefore, present a moderately enlarged image of the eye-ground, leaving out many.con-

1 Sketch-book,p. 4: "The ophthalmoscopist who possesses any proficiency in the art of drawing will notice, incidentally, that the drawing of these sketches sharpens his powers of observation and impresses the picture more firmly on his memory. If we intend to draw a thing, we are forced to observe it much more accurately." 
ditions which naturally confuse the beginner and are unnecessary for the expert, such, for instance, as the reflexes of the retina and vessels, and the delicate, irregular mosaic arrangement of the pigmentation of the fundus, etc. These details, which are seen chiefly in the upright image, are very troublesome to reproduce in lithographic plates, especially when one considers that the lithographic reproduction of even a small image of the eye-ground presents great difficulties. I therefore directed all my efforts to obtaining in every instance an absolutely faithful reproduction of whatever is typical or pathologic. The pictures have been so colored that when seen in daylight they produce the impression received in looking at the eye-ground with artificial light; in other words, the whitish parts (optic nerve, etc.) when seen by daylight are not as yellow as they are in the original sketches, which were made by artificial light. In an artificial or yellow light a moderately deep yellow color appears white, hence pictures prepared by artificial light are too yellow and must be made more white if they are to produce the same impression by daylight. The pictures in Jäger's large atlas are colored in such a way that they appear correct by artificial light; but if they are examined by daylight the unnaturally yellow color of the lighter portions becomes a disturbing factor. My pictures, on the contrary, are a little too white by artificial light, but to judge from all the pictures in ordinary use this is neither a serious nor a disturbing defect. In his smaller atlas Jäger, in accordance with the custom which arose later, also made his pictures more white; but, it is to be remembered that Jïger's pictures were prepared with Helmholtz's ophthalmoscope, so that in many of them the gray, and particularly the green, shades in the optic disk are unusually prominent (for instance, in the picture of glaucoma). In my pictures the coloring is such as it appears with the stronger ophthalmoscope, which, for reasons to be given later, I use exclusively in the examination of the eye-ground. 


\section{DESCRIPTION OF THE OPHTHALMOSCOPE.}

Before the iurention of the ophthalmoscope by $\mathrm{H}$. v. Helmholtz, in 1851, our knowledge of the interiol of the eye in the living subject was as dark as the pupil itself. Until the invention of our beautiful instrument the world had no conception of the interior of the eyeball as we now see it with the ophthalmoscope in all its lucidity and wealth of coloring. Most of the pathologic alterations visible by means of the ophthalmoscope were not even properly known at that time.

Why is it that we cannot without the aid of an appropriate instrument penetrate into the depths of the eye? Why is it that the interior of the eye and the pupil appear black except in a man or animal devoid of pigment (albino)?

The conditions are exactly the same as when we look into a camera obscura-as, for instance, a photographic apparatus open and ready for the reception of an imagealthough we know that on the sensitive white plate at the back of the instrument there is an accurate colored image of the objects in front of it, we cannot see a trace either of the white plate or of the picture. To us the interior of the camera and the opening through which the rays of light enter appear black, and all that we see in the lens occupying this opening is a minute image of ourselves, such as may be seen in the cornea of the eye.

No one acquainted with the most elementary laws of optics will have any difficulty in understanding why the pupil appears black, and why, without special instruments, we are unable to look into the interior of an eye. As in the camera obscura, the refracting system of the eye, which is a double lens consisting of the cornea and aqueous humor, throws a reduced inverted image on the retina. This image appears sharply outlined if the eve is properly focussed for the object, and blurred-that is to say, in diffusion circles-when the eye is not properly focussed, just as in the photographic camera. 
Now we learn from one of the laws of the refraction of lenses that object and image bear a definite relation to each other-they are said to be reciprocal or conjugateso that they may be interchanged withont necessitating any alteration in the dioptric srstem or in the distances from the lens to the object and to the image. If in a dark room we obtain on the disk of ground glass a clear image of a candle held at a distance of one meter in front of the object-lens, and then put the light in the place of the ground glass, we will get a clear image of the flame by holding the ground glass where the light originally was, that is to say, one meter in front of the lens. In other words, we may reverse the positions of the candle and of the ground glass, and in each case obtain a sharp image of the candle, providing we retain the original distances-in the former case bchind, and in the latter in front of the lens. The rays of light emitted by the image on the ground glass, after leaving the apparatus, all return to the flame of the candle. But as our eyes do not send out any rays of light when we look into a dark chamber, they cannot receive any rays in return ; hence the opening of the camera and the object-lens appear dark, and in the same way the opening of the eye into which we are looking; in other words, the pupil also appears dark.

If, however, rays of light are sent out from the observer's eye, the rays which enter the observed eve return to the eye of the observer, and the pupil of the observed eye appears red like that of an albino. Before the invention of the ophthalmoseope an erroneous theory was current that the pigment of the fundus absorbed all the light that enters the eve, and the pupil of a pigmented eye therefore appeared black. If the eyc-ground of a normal eye is illuminated by rays energing from the observer's eye, renough rays will be reflected by the eye-ground, which even in a pigmented eve is not entirely black, to enable the observer to get a clear image of the other eye. On the other hand, the pupil of an albino is red, not because of any want of pigment in the fundus, but because of the 
absence of pigment in front, so that the rays of light pass through the iris, sclera, and choroid, and illuminate the eye at every point instead of only at the focus of the reflecting media. In such eyes there is no reciprocal relation between the object and the image; the rays emerge from the pupil in all directions, and the latter appears in a red light because the eye-ground of the albino (in man, in the white rabbit, etc.) is colored red by the numerous blood-vessels of the choroid. If in the albino the light is prevented from entering the eye in this abnormal way through the unpigmented tissues outside the pupil, and the pupil is then examined, it will appear as dark as in the ordinary individual. This can be done by holding immediately in front of the albino's eye an opaque cup with an opening corresponding in size to the pupil. If the light is allowed to enter only through this opening and through the pupil, the latter will appear as black as that of a pigmented eye.

Now we can by means of any simple device, and best by means of the ophthalmoscope, send out light from our own eye. Even an ordinary glass disk held in front of the eye, by virtue of its reflecting qualities, will direct into the eye the rays emanating from a light standing to one side. If the light is placed to the left of the person examined, and the glass disk is turned slightly toward the lamp, so that the reflection of the light falls on the observed eye, the pupil will immediately appear red when seen through the piece of glass (cf. Fig. A). As the rays coming from the lamp $(L)$ are in part reflected into the observed eye by the glass disk, they enter it in such a way that they appear to come from a point behind the observer's eye, that is, from the point $L_{2}$, which would correspond to the reflected inage of the lamp. Since they enter the observed eye they illuminate the eyeground. If the eye is focussed for the distance of the reflected image of the lamp, a clear image of the lamp will be produced on the fundus; but if the eye is not focussed for this distance, the image will be blurred. In 
any case a certain portion of the eye-ground of the observed eye is illuminated and thereby enabled to send back rays of light. These rays return to the object-in this case the flame of the lamp. As the disk of glass not only reflects but also transmits light, a portion of the rays returning from the observed eye pass through the glass and enter the observer's eye, while the remainder are reflected toward the lamp and lost to the observer.

But the amount of light entering the observer's eve in this way is very slight and the illumination of the pupil

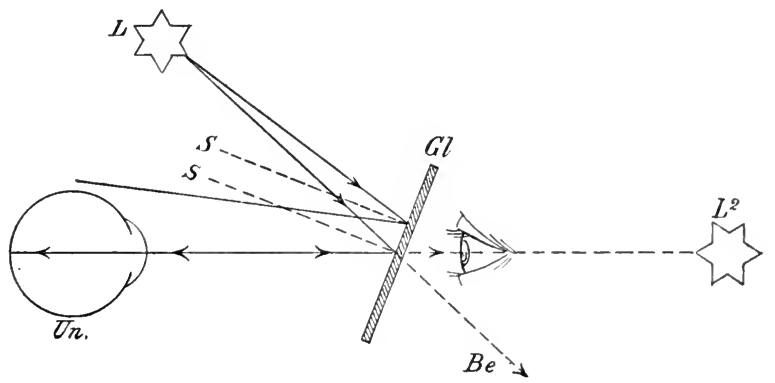

FIG. A.-Illumination of the eve under examination ( $U n$.$) by means$ of the glass disk, Gl.; two of the rays coming from a point in the light $(L)$ are shown; one of these ray, after being reflected by the glass plate, misses the eye; part of the other ray lasses through the glass ( $(r l$.$) and is$ lost to the observer (Be.), while the remainder is reflected in to the eve of the person examined ( $C h$.), returns by the same path, and enters the eye of the observer in the direction of $L_{2}$, the reflected image of the eye; $s, s_{\text {, }}$ perpendiculars to $G l$.

of the observed eve correspondingly weak. The light may be increased by adopting Helmholtz's plan of laying several glass disks one upon the other, but even with such an arrangement the amount of light entering the observer's eye is comparatively small. A much better result is obtained by throwing more light into the observed eye, so that the eye-ground is more powerfully illuminated and accordingly sends back more light, and, secondly, by allowing the returning rays to enter the observer's eye 
without being weakened; that is to say, through a hole in the mirror.

Accordingly, Helmholtz's disks were soon replaced by a true mirror which throws much more light into the eye. By scraping away the coating from a small spot in the center an opening was obtained for the returning rays to enter the observer's eye. The amount of light entering the eyes, both of the observed and of the observer, may be still further increased by using a concave mirror which concentrates the light before throwing it into the observed eye, and by making an actual opening at the center of the mirror, so that the returning rays meet with even less resistance in their passage through the mirror.

By thus illuminating the eye with the rather weak Helmholtz ophthalmoscope, or better, with the somewhat stronger but still comparatively weak plane mirror, or, best of all, with a powerful concave mirror, we can obtain a distinct view of the otherwise invisible eyeground. As the illuminated fundus sends out rays of light which enter the observer's eye in the manner explained, it is evident that the observer obtains in his own eye a clear image of the eye-ground observed. The fundus of the observed eye is converted into a luminous object which we can see like any other object in the outside world. Strictly speaking, however, we see the eyeground as we see an object through a magnifying glass, the reflecting media of the observed eye forming a lens through which we see the individual portions of the eyeground under a high magnification. Some persons require additional aids to obtain a elear view of the eye-ground by the method just deseribed.

This method of examination is known as the direct methor or the methorl with the erect image. It is called the direct methor beeause it enables a normal eye to examine another normal or hypermetropic eye without any additional aids. It is called the examination with the erect image because we see the eye-ground right side up like any other object in the outer world. 


\section{EXAMINATION IN THE ERECT IMAGE.}

Without certain devices to alter the direction of the rays of light emerging from the patient's eye, this method of examination is often impossible, and this brings us to a further and very important feature of ophthalmoscopic examination.

It appears that the ophthalmoscope not only enables us to see the things which are to be seen in the eyeground, but also furnishes us with the means of accurately measuring the refracting power and the structure of the observed eye; and these data are most valuable, since they are entirely objective and render us independent of the patient's statements. 'To do this we use our own eye, the refractive system of the eye examined, and possibly certain convex or concave lenses, and thus calculate in the simplest possible manner the optical power of the observed eye.

To understand this it is necessary first to consider the manner in which the entering rays leave the eye in the ease of a normal, a myopic, and an hypermetropic organ.

As has been explained above, the rays of light always return to the point from which they have come and on which the eye is therefore focussed-in the above example the flame of a candle fixed at a distance of one meter in front of the eye. In this case the eye is accommodated for the distance of one meter. An emmetropic eye without accommodation is, of course, focussed for infinity ; that is to say, for parallel rays. The fundus of such an eye, therefore, reproduces a clear image of all objects sending out parallel rays of light; in other words, of all distant objects. As the liameter of the pupil is very small, an object may be considered distant if it is 3 to 5 "meters away from the eye; that is to say, the error of considering rays coming from such a point as parallel rays is infinitesimally small. When, therefore, an emmetropic eye looks into infinity or looks at an object 3 to 5 meters away, the rays of light leaving the 
illuminated eye-ground also return to infinity and are therefore parallel (cf. Fig. C). It follows that if we examine an emmetropic eye by the above-described direct method, the rays of light emerging from the eye immediately form a clear image of the fundus on the observer's eye, providing the observer also looks into infinity; in other words, adjusts his eye to parallel rays. If the observer accommodates instead of looking into infinity, he will obtain a blurred image of the observed eyeground; but as in order to look into an eye one has to get as near to it as possible, just as when looking into a room through a key-hole, it is somewhat difficult to relax the accommodation. This is one of the greatest difficulties encountered by the beginner in attempting to examine by the direct method.

It is, however, absolutely indispensable if he wishes to obtain a correct measurement of the refraction of the observed eye; in other words, the observer's eye, in order to measure the refraction of another eye, must relax its accommodation completely, because it is impossible to calculate the increase in the refractive power of the observer's eye due to the accommodation. We can measure our own accommodation only when we know for what distance, that is to say, for what degree of divergence of the rays entering our own eye, it is focussed; but the very object of an examination by the direct method is to determine the divergence of the rays of light which leave our own eye.

It follows from what has been said that the observer must know the exact refractive power of his own eye in order to be able to measure that of the patient's eye. If the observer is emmetropic, he gets a clear image of the eye-ground of the patient's eye without any further appliances. If he is myopic, he will obtain a blurred image, as everything that sends out parallel rays, or in other words, is at an infinite distance from his eye, appears blurred. To see distant objects the myope needs spectacles with concave lenses; hence to examine 
by the direct method the myope must also use a correcting glass, which he places behind the opening in the ophthalmoscope, if he wishes to obtain a clear image of the eye-ground of the emmetropic eye.

If the observer is hypermetropic, he is in the same position when he attempts to examine by the direct method as when he looks into infinity; he must, since his eye is not focussed for parallel rays, either wear a correcting glass with convex lenses or accommodate. But as he is not allowed to resort to the latter expedient, he must, during the examination, use a glass which completely corrects his hypermetropia. His hypermotropia must be completely corrected, so that he may not be foreed to compensate for some unknown degree of hypermetropia by using his aceommodation. This is particularly difficult for the hypermetrope, because he has beeome so accustomed to correet his optieal error by accommodating that he finds it very difficult to substitute a correcting lens for his power of accommodation. He continues to accommodate even when looking through the correcting lens-at least to some extent-and is accordingly over-corrected. This is particularly the case in high grades of hypermetropia when the individual is young and the power of accommodation is well preserved.

Surgeons with moderate hypermetropia are, therefore, often compelled to resort to some other means of measuring the refraction if they do not wish to depend on the patient's statement-either to Sehmidt-Rimpler's method by means of the inverted image, or to the shadow-test. These two methods will be discussed later.

We have assumed up to this point that the person examined is emmetropic and that the observer is emme"tropic, myopic, or hypermetropic. What is the condition of affairs when it is desired to examine an eye with abnormal refraction? We must first determine in what way the rays emitted by an illuminated eye-ground leave the eye in the case of myopia or hypermetropia. Again, 
we assume that the ametropic eye under examination looks into infinity, so that the accommodation, which we cannot compute, may be disregarded in the calculation. The patient, whether he be emmetropic or ametropic, must always relax his accommodation while his refraction is being taken.

\section{Measurement of the Myopic Eye.}

A myopic eye, when it is not accommodating, is focussed for a point at a finite distance, depending on the degree of myopia. This point is known as the far point (punctum remotum). It is the farthest point at which an eye of this kind sees distinctly. Only those rays which come from this point are collected on the retina and form there a distinct image, when the eye is looking into infinity without accommodating. Rays coming from a more remote point are focussed in front of the retina, and the image formed on the retina is blurred. The reason that the image is formed in front of, instead of on the retina, as in the normal eye, is usually that the myopic eye is too long (cf. Fig. D) or that the refracting power of the lens is too great. Excessive length of the eyeball is the chief cause of myopia. In order to see distant objects distinctly, the myopic eye must be corrected by means of a concave lens which weakens the refraction; so that the entering rays are focussed on the retina, behind the focus of the lens.

Let us now consider how the rays emanating from an illuminated portion of the eye-ground in a myopic eye leave the eye after traversing the refractive media in the contrary direction. If the eye does not accommodate, they will tend to converge at the far point (cf. Fig. D), since the far point is at a finite distance from the eye ( $a^{\prime}, b^{\prime}$, plane of the far point). If the far point is situated at a distance of one meter from the eye, the degree of myopia is equivalent to a lens of one-meter focus (diopter), and the error in such a case is corrected by means of a lens having a focal distance of one meter. If the far point is situated at 0.5 meter from the eye, the 
error is twice as great and the correcting glass must be twice as strong; that is, a glass equivalent to two meterlenses or diopters; or, in other words, a lens having a

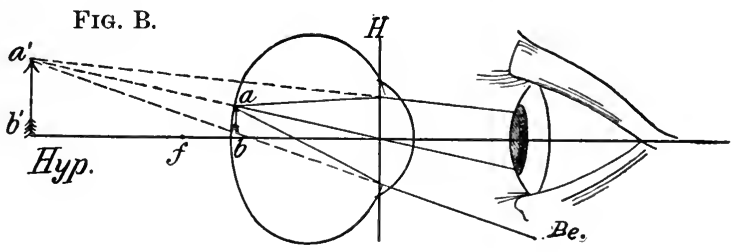

Fig. C.
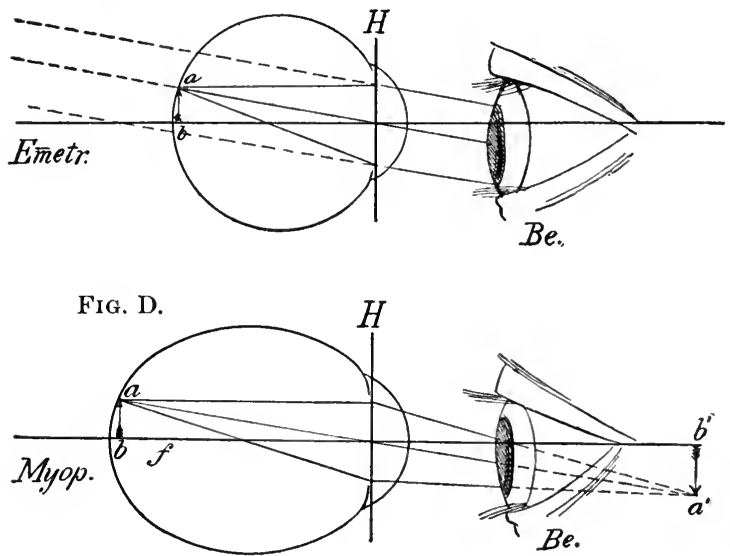

FIGS. B, C, D.-Examination in the erect image when the eye examined is hypermetropic, emmetropic, or myopic. In each figure three rays are shown emanating from a luninous point on the eye-ground. In hypermetropia they diverge after leaving the eye, in emmetropia they are parallel, in myopia they converge: $f$, the posterior focus; $H$, principal plane of the dioptric system of the examined eye; Be., observer. The ophthalmoscope is not shown.

focal distance of 0.5 meter. If the distance of the far point is $\frac{1}{4}$ meter $=25 \mathrm{~cm}$., the degree of myopia is four times as great $=4$ diopters, and the correcting glass must 
have a focal length of $\frac{1}{4}$ meter $=25 \mathrm{~cm}$., etc. In the last case, for instance, the myopia is corrected by a concave lens of $4 \mathrm{D}$ (with a focal distance of $25 \mathrm{~cm}$.), because such a glass when held immediately in front of the eye renders parallel rays coming from infinity so divergent that they appear to come from the far point of the eye, which is at a distance of $25 \mathrm{~cm}$.; for a concave lens of $4 \mathrm{D}$ lends such a degree of divergence to entering parallel rays of light that they seem to come from the focus of the lens $(=25 \mathrm{~cm}$.).

It follows, therefore, that the rays of light leaving a myopic eye converge to the far point. If, therefore, a normal eye is placed behind the ophthalmoscope it will not see anything of the eye-ground, since it is not focussed for converging rays of light, for converging rays do not occur in nature without a special cause. The examiner's eye, therefore, requires an additional lens behind the ophthalmoscope of such a strength as completely to correct the myopia of the examined eye; or, in other words, to render parallel the rays which emerge from the examined eye, since the normal eye is focussed for parallel rays of light. The degree of myopia of the examined eye can, therefore, be found in this way by selecting the weakest concave lens with which it is possible to get a distinct image of the fundus. The weakest concave lens must be selected so as to eliminate the examiner's own accommodation, for he could see quite distinctly with a stronger concave lens, because his power of accommodation would enable him to neutralize the excessive concavity of the lens.

If the observer is myopic, he will need, in order clearly to see the fundus of another myopic person, a concave lens strong enough to correct both his own and the other's myopia. If, for example, he finds that a lens of $5 \mathrm{D}$ is the weakest lens with which he can see the eye-ground clearly, and if he himself has a myopia of two diopters, the examined eye has a myopia of $3 \mathrm{D}$.

If, on the other hand, the observer is hypermetropic 
to an extent, let us say, of two diopters and finds the correcting coneave lens to be one of $5 \mathrm{D}$, he must add his own hypermetropia, and the patient's eye in that case has a myopia of $7 \mathrm{D}$. An emmetropic observer in this case would need a concare lens of $7 \mathrm{D}$, but the hypermetrope needs one of only $5 \mathrm{D}$, because his own correcting convex glass of $2 \mathrm{D}$ neutralizes the effect of a concave glass of $2 \mathrm{D}$. He would also need -7 if he looked through the ophthalmoscope with his own correcting lens of $2 \mathrm{D}$. But it is better to have only one lens behind the ophthalmoscope, hence he will need only - 5 instead of -7 to correct the myopia of the examined eye.

\section{Measurement of the Hypermetropic Eye.}

Having now considered cases where the examined eye is either emmetropic or myopic, we must consider the possibility of its being hypermetropie.

Again, we must consiler how the rays of light emerge from an hypermetropic eye when it is illuminated with the ophthalmoscope. An hypermetropie ere without acemmodation is focussed neither for parallel nor for divergent, but for convergent rals of light (ef. Fig. B); that is to say, only convergent rays of light are eollected on the retina to form a distinct image. Parallel rars of light entering the eye unite to form an image behind the retina at the point $f$, either because the axis of the hypermetropic ere is too short or because its refractive system is too weak, as, for instance, when the lens is absent. In either ease the refractive srstem of the bypermetropie eye is insufficient as compared with its axis, and therefore requires a re-enforcing lens. The hypermetropic eye can, by exerting its aceommodation, increase its refractive power by increasing the refraction of the lens, so as to bring the image forward and on to the retina. It differs from the emmetropie eye in the fact that it has to accommodate even when looking into infinity, but this ean be obviated by the use of a convex lens. As a rule, the visual error in the hyperme- 
tropic eye is only partially corrected by a lens, the individual correcting the remainder himself. In fact, it is impossible in youthful subjects, whose power of accommodation is vigorous, to find the degree of hypermetropia by means of correcting lenses. Such persons always accommodate more or less when they fix an object, and they will accept only a convex glass which corrects a portion of their hypermetropia. This portion is called the manifest hypermetropia of the individual, while that portion which is corrected and concealed by the accommodation is known as the latent hypermetropia. As the individual grows older and the power of accommodation diminishes, the manifest hypermetropia increases. If the accommodation is entirely removed, either by age or by a drug (atropin, homatropin), the total hypermetropia becomes evident.

The examination in the erect image furnishes a very convenient method of determining an individual's total hypermetropia without artificially paralyzing the accommodation; for if the hypermetrope, even though he be young, does not focus his eyes, but looks into the distance in the dark room, he will not accommodate during the examination with the ophthalmoscope, and the rays of light leaving the eye will follow the direction given them by the structure of the media ; that is to say, they diverge. The degree of divergence is proportional to the degree of hypermetropia and inversely proportional to the distance of the negative far point behind the eye. If the hypermetropia is such that the eye is focussed only for rays which converge at a point 0.5 meter behind the principal plane of the eye, the error is corrected by a convex lens having a focal distance of 0.5 meter, or a lens of $2 \mathrm{D}$. The hypermetropia is $2 \mathrm{D}$. If in order to be collected on the retina the rays require a greater degree of convergence, for example, to a point only $25 \mathrm{~cm}$. behind the principal plane of the eve; in other words, if the far point is 25 $\mathrm{cm}$. (=- $\frac{1}{4}$ meter $)$ behind the eye, the error is twice as great. The hypermetropia is $4 \mathrm{D}$, and the correcting lens must 
be of equal strength, unless the individual when looking into infinity accommodates to the extent of $4 \mathrm{D}$. A lens of $+4 \mathrm{D}$ held in front of such an eye will, therefore, give to the rays coming from infinity the neeessary degree of convergence; for a convex lens of $4 \mathrm{D}$, held immediately in firont of the eye, refracts parallel rays entering the eye in such a way that they converge toward the focus of the lens $-25 \mathrm{~cm}$., which is also the far point of the eye, as we may disregard the distance from the lens to the principal plane of the eye.

The same correcting lens will render the divergent rays emerging from the eye parallel, since they come from the focus of the lens. Approximately the same result is obtained by holding the correcting lens behind the opening in the ophthalmoscope. An observer with normal vision looking into such an hypermetropic eve will be able to see the fundus distinctly. He could, of course, see it without a correcting lens, but only by using his accommodation to the extent of $4 \mathrm{D}$ in the present example, which would be a source of error in obtaining the measurement of the eve.

If the observer himself is hypermetropic, he must combine his own correcting lens with that required by the examined eye. To determine the error of the examined eye he must subtract his own correcting lens from the convex lens used. It is best in this case to use the strongest lens with which the fundus can be seen distinctly, so as to avoid neutralizing a certain portion of the hypermetropia by one's own accommodation.

If, on the other hand, the observer is myopic, he needs a weaker correcting lens than does the observer with normal vision, because his own error neutralizes the error of the examined eve. If, for instance, the observer's eye is focussed for the rays coming from a distance of $20 \mathrm{~cm}$. (myopia 5 D), his eye withont aceommodation is focussed for rays emerging from the eye of an hypermetrope of $5 \mathrm{D}$, and he therefore needs no glass. The hypermetropia of the patient's eye in such a case is equivalent to the exam- 
iner's myopia. If the observer requires a convex lens he must add to the value of this lens the number of diopters of his own myopia. If, for instance, his myopia amounts to $3 \mathrm{D}$ and he needs $\mathrm{a}+2 \mathrm{D}$ lens to see the fundus, the patient has an hypermetropia of $5 \mathrm{D}$. If a myope of $7 \mathrm{D}$ needs only a $-3 \mathrm{D}$ lens, the patient has an hypermetropia of $4 \mathrm{D}$.

The beginner in ophthalmoscopy should always remember (cf. Figs. B, C, D) that rays emerging from an emmetropic eye are parallel, those from a myopic eye convergent, and those from an hypermetropic eye divergent; and, conversely, that the examining eye without accommodation is focussed for parallel rays of light if it is emmetropic ; for divergent rays, if it is myopic; and for convergent rays, if it is hypermetropic.

From what has been said we may deduce the following general rules :

If in order to see distinctly the eye-ground of another's eye an ametrope nceds-

(1) A lens of the same kind as that which corrects his own ametropia, but of a greater strength, he must subtract the number of his own diopters from the diopters of the lens user.

(2) If the required lens is of the same kind, but from 1 to 10 diopters weaker than that which corrects his own ametropia, the refractive error of the examined eye is of the contrary kind and amounts to from 1 to 10 diopters. (Examples: If the observer has a myopia of 6.0 and needs - 5.0, the eye examined has an hypermetropia of 1.0. If the observer needs a 4.0, the eye examined has an $\mathrm{H}$. of 2.0 , etc. If the observer has an $\mathrm{H}$. of 4.0 and needs a +3.0 , the eye examined has a $M$. of 1.0 , etc.)

(3) If the required lens is of the contrary kind from that required to correct the observer's ametropia, the eye examined has the contrary refraction, and the amount of the error will be equal to the number of diopters of the required lens plus the number of diopters of the ob- 
server's correction, it being immaterial whether the lens found is stronger or weaker than the examiner's own lens. Examples: If the examiner has M. 5.0 and needs a +3.0 lens, the patient has an hypermetropia of 8.0. But, if the examiner has H. 3.0 and needs a -4.0 lens, the patient has a myopia of 7.0. If the examiner has H. 3.0 and needs a -2.0 lens, the patient has a myopia of 5.0. If the examiner has M. 3.0 and needs a +5.0 lens, the patient has an hypermetropia of 8.0.

The refraction can be determined even more rapidly in the erect image by means of a simple formula based on the fact that the strength of the lens found with the ophthalmoscope- $(\mathrm{Sp})$-is equal to the sum of the ametropia of the examiner $(\mathrm{A})$ and that of the patient $(\mathrm{x})$. Hence the formula :

$$
s_{p}=A+x .
$$

Hence, if the examiner's ametropia is deducted from the strength of the lens found with the ophthalmoscope (Sp), the remainder is the patient's ametropia :

$$
\mathrm{Sp}-\mathrm{A}=\mathrm{x} \text {. }
$$

In carrying out this subtraction it must be remembered that the subtraction of a negative quantity is equivalent to addition.

\begin{tabular}{|c|c|c|c|}
\hline $\begin{array}{l}\text { Ophtalmo- } \\
\text { scopic lens } \\
\text { (Sp). }\end{array}$ & $\begin{array}{l}\text { Examiner's } \\
\text { ametropia } \\
\text { (A). }\end{array}$ & $\begin{array}{l}\text { Calculation } \\
\text { Sp-Ax. }\end{array}$ & $\begin{array}{l}\text { Patient's } \\
\text { ametropia } \\
(\mathbf{x}) .\end{array}$ \\
\hline $\begin{array}{l}+5 \mathrm{D} \\
+5 \mathrm{D} \\
-5 \mathrm{D} \\
-5 \mathrm{D} \\
+2 \mathrm{D} \\
-2 \mathrm{D}\end{array}$ & $\begin{array}{l}2 \text { D. H. }(+2) \\
2 \text { D. II. }\left(-\frac{2}{2}\right) \\
2 \text { D. II. }\left(-\frac{2}{2}\right) \\
2 \text { D. II. }\left(+\frac{2}{2}\right) \\
5 \text { D. II. }(+5) \\
5 \text { D. II. }(-5)\end{array}$ & $\begin{array}{l}(+5)-(+2)=5-2=+3 \\
+5)-(-2)=5+2= \pm 7 \\
-5)-(-2)=-5+2=-3 \\
(-5)-(+2)=-5-2=-7 \\
(+2)-(+5)=-2-5=-3 \\
(-2)-(-5)=-2+5=+3\end{array}$ & $\begin{array}{l}+3(2 \text { D. Iyp. }) \\
+7 \text { (7 D. Iyp.) } \\
+3(3 \text { D. My. } \\
-7 \text { (7 D. My.) } \\
-3(3 \text { D. My. }) \\
+3 \text { (3 D. IIyp.) }\end{array}$ \\
\hline
\end{tabular}

\section{EXIMPLES.}

- In order to be accurate in determining the refraction of an eye the following points must be observed: The dioptric conditions of that portion of the eye-ground which corresponds to the point of clearest vision-viz., the macula lutea or fovea centralis of the retina-must 
be determined. Hence in measuring the refraction of an cye the examiner must obtain a good view of that portion of the eye-ground. Now the macula lutea is the most difficult portion to see, because when we attempt to examine it the pupil contracts and the reflection of the light on the cornea is most disturbing.

Besides, it is not very easy to determine whether the image is clear when we look into the macula lutea, because there is a lack of conspicuons markings, the retinal vessels being at this point extremely small. All that can be seen is a fine stippling of the eye-ground due to the somewhat variable amount of pigmentation of the cells forming the pigmented epithelium of the retina. The darker the eye-ground, the more uniform it usually appears and the more difficult it is to focus accurately. It is true that in dark eye-grounds, especially in young individuals, a small bright sickle or ring is seen, due to the reflection of the light at the bottom of the fovea centralis, and although this reflex is a little in front of the retina, the difference is so slight that it need not be considered in calculating the refraction. If the forea reflex is too indistinct to be utilized in this way, it is best to look for the vessels which pass from the optic nerve toward the macula lutea or for a vessel coming from above or below and running toward the macula.

The beginner will do well to confine himself to the temporal margin of the optic disk, as it is more clearly outlined, and the presence of blood-vessels which pass over it toward the macula (cf. Figs. 1, 4, etc.) makes it possible to obtain measurements in the various meridians. In this way the so-called astigmatism of the eye can also be measured.

\section{Measurement of Astigmatism.}

The term astigmatism is applied to an abnormal refractive condition of the eye in which one or more of the refractive surfaces, instead of being spherical or slightly 
parabolie, have a greater curvature in one meridian than in the meridian at right angles to it. When a pencil of rays diverging from a luminous point impinges on a refractive surfice with an equal curvature in all its meridians, as, for instance, a hemisphere like one of our glass lenses, the rays are all united in one point, disregarding

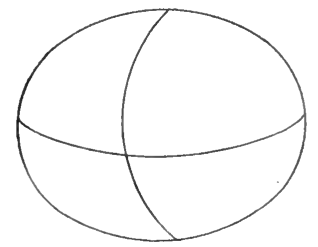

Fig. E.-Refractive surface, with a greater curvature in the vertical than in the horizontal meridian.

those which strike the lens too near the margin, known as marginal rays. But if the pencil of rays impinges on a surface having a weaker curvature in the horizontal than in the vertical meridian (cf. Fig. E), the rays passing through the rertical meridian are more strongly refracted than those passing through the horizontal. Hence the former rays are brought to a focus at a point nearer the refractive surface than are the latter. The surface has two foci, and rays emanating from a luminous point ("homocentric" pencil) are not united at any one point behind the refracting surface, hence the name

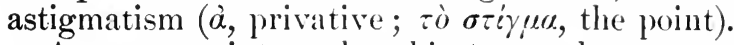

As every point on the object must have a corresponding point in the image in order that a clear image may be produced, it follows that an astigmatic surface can produce only a distorted image. Hence the image on the retina of an astigmatic eye will be distorted whether the abnormal curvature is in the lens or in the cornea; and, eonversely, when such an eve is examined with the ophthalmoscope, the markings on the eye-ground (optic disk, vessels of the retina, ctc.) appear blurred and cannot be brought out clearly with any additional spherical 
lens. A lens with a curvature in only one direction-a so-called cylindrical lens-is required. A lens with no curvature in the vertical, and a corresponding positive curvature in the horizontal meridian will, in the above example (see Fig. E), correct the abnormally weak horizontal curvature of the refracting surface. Some ophthalmoscopes permit of the use of such cylindrical lenses, but they are not absolutely necessary for the measurement of astigmatism. With a little practice it is usually quite possible to measure the refraction for every individual meridian. [Dr. B. Alex. Randall has designed an ophthalmoscope which carries a disk, by means of which cylindrical lenses can be rotated behind the sight-hole, which is of great practical value.-ED.]

The student should remember that the vertical meridian of a spherical or sphericocylindrical (astigmatic) lens corrects the horizontal and not the vertical lines of the object, while the horizontal meridian corrects the vertical and not the horizontal lines. If, therefore, the horizontal vessels running from the optic disk toward the macula are seen distinctly, it shows that the refraction of the vertical meridian of the refractive system has been correctly determined. Another glass will be required to obtain a clear image of the vertical lines (those, for instance, that run into the macula from above or below), or of the temporal margin of the optic disk which at one point is approximately vertical. This glass corresponds to the refraction of the horizontal meridian of the refractive system.

It happens sometimes that the principal meridians intersect each other obliquely instead of at a right angle. In that case the use of a certain lens will enable the observer to see the vessels rumning outward and upward near the optic nerve, while those which run outward and downward appear quite indistinct. To see the latter another glass will be required, with which again the vessels running outward and upward will appear indistinct. In measuring the astigmatism it is of the 
greatest importance that both the examiner and the patient relax their accommodation.

Care must also be taken not to look through the lens obliquely. This should always be aroided as much as possible, otherwise the observer might discover astigmatism where none is present, or even, under certain conditions, correct astigmatism without knowing it ; for by looking obliquely through an ordinary spherical lens we get the effect of an unequal refraction exactly similar to the regular astigmatism now under discussion.

We also speak of irregular astigmatism. This occurs when a refractive surface has an irregular curvature, as, for instance, when the surface is uneven. The image in this case is blurred and cannot be made distinct for any length of time by any means in our power. Irregular astigmatism is usually due to inequalities in the surface of the cornea corresponding to more or less marked opacities. These defects in the cornea can usually be recognized by means of lateral illumination.

Finally, in measuring refraction with the ophthalmoscope by the direct method, the distance between the observer's eye and that of the patient must be considered.

It should always be as short as possible, otherwise the correcting lens of the ophthalmoscope is too far removed from the eye examined, and its refractive power is altered. The power of a concave lens is lessened by increasing its distance from the eye; and, conversely, a convex lens is more powerful when placed at a greater distance from the eye, which explains why old people often push their spectacles down to the tip of the nose to make them stronger.

This error requires correction in strong lenses, but - may be disregarded in weak glasses, espeeially as the spectacles to be ordered will always be worn at some distance from the eye.

In using strong lenses it is, therefore, always to be 
borne in mind that the correcting lenses found with the ophthalmoscope will be too strong in myopia and too weak in hypermetropia, if the observer's eye is too far away from the eye examined. In such a case, therefore, the myopia of the eye examined is weaker and the hypermetropia stronger than the lens in the ophthalmoscope.

\section{Size of the Ophthalmoscopic Field of Vision.}

Another reason for coming as close as possible to the eye under examination is that it is easier in that way to look into the eye through the comparatively narrow pupil. By coming as close to the pupil as possible the observer is able to see a larger portion of the eye-ground without changing the relative positions of his own and the patient's eye ; in other words, the ophthalmoscopic field of vision is increased. If the pupil is very small, it must be dilated either by shielding the other eye from the light, or by means of drugs-homatropin in 2 per cent., or euphthalmin in 5 per cent. solutions. From 2 to 3 drops instilled within five minutes usually suffice to produce the desired amount of dilatation within twenty minutes. The use of atropin for this purpose is to be avoided as much as possible, as its action lasts too long and may produce increased intraocular tension (glaucoma), especially in elderly people. [No mydriatic is entirely free from the danger of producing glaucoma; even euphthalmin has caused this disease, witness a case reported by Knapp. Euphthalmin mydriasis may be neutralized by the instillation of a pilocarpin solution ( $\mathrm{gr} . \mathrm{j}-\mathrm{f} \tilde{\mathrm{s}} \mathrm{j})$ before the patient is dismissed.-ED.]

An experienced observer will very rarely need to dilate the pupil artificially in a normal eye unless he particularly wishes to examine the macular region. For this purpose artificial dilatation is often indispensable, especially in elderly people, who usually have smaller pupils than young persons. For similar reasons the opening in 
the ophthalmoscope should not be too small. The most desirable width is $3.5 \mathrm{~mm}$. [Some surgeons prefer a smaller opening-e. $g$., Jackson recommends that the aperture in the mirror shall be $2 \mathrm{~mm}$. in diameter.-ED.]

The size of the ophthalmoscopic field further depends on the refraction of the eye under examination. It is greatest in hypermetropia, because, as we have seen, the rays diverge after leaving the eye; smaller in enmetropia ; and still smaller in myopia, because the emerging rays converge.

Another factor that influences the size of the ophthalmoscopic field in the direct method is the size of the flame used in the examination, particularly when a strong concave mirror is used, one, for instance, with a focal distance of $16 \mathrm{~cm}$., which is also employed in the indirect method, soon to be discussed. With a mirror of this kind one often gets an inverted image of the flame on the eye-ground while everything else is indistinct. Beginners will, therefore, do well not to use too small a flame.

If it is desired to illuminate a very large field a concave mirror with a very short focal distance may be used. This third mirror, however, complicates the instrument unnecessarily.

This brings us to the choice of a mirror in the direct method. Besides the strong concave milror just deseribed, which is best for beginners, we may select either a plane mirror or a concave mirror of about $16 \mathrm{~cm}$. focal distance, which is also used in the indirect method. The latter is decidedly to be preferred in the examination of the macular region, which on aceount of its dark color usually reflects very little light. The macula is not suf- ficiently illuminated by the plane mirror, although the latter has the advantage of not contracting the pupil quite so much. 


\section{EXAMINATION BY THE INDIRECT METHOD.}

This method, first proposed by Rüte, consists in illuminating the eye-ground by means of a concave mirror and holding a strong convex lens (13-20 D) at the near point; that is to say, at a focal distance of 5 to $7.5 \mathrm{~cm}$. from the patient's eye, so as to unite the rays of light as they emerge from the eye and produce a true inverted image (cf. Fig. F. To save room the lens and the eye of the observer are shown somewhat nearer the patient's eye than they ought to be). In this method the observer sits at a greater distance from his patient, and sees the

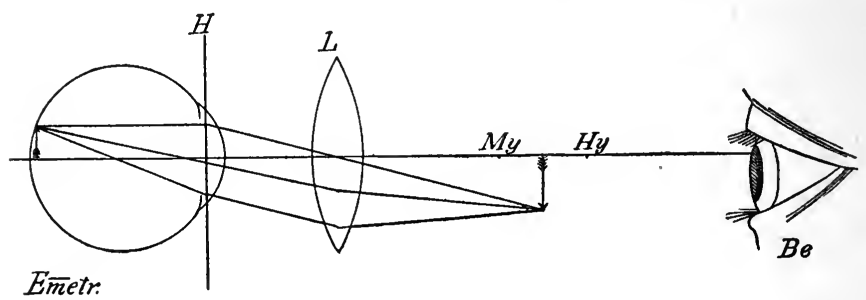

FIG. F.-Examiuation of an emmetropic eye by the indirect method: the parallel rays of light coming from the eye are focussed by the lens $(L)$, and form a true inverted image which is directly seen by the observer $(B e) ;$.$H , principal plane; M y$. and $H y$., the respective points where, in myopia and hypermetropia, the inverted image is formed. The ophthalmoscope is not shown.

inverted image very distinctly by looking through the opening in the ophthalmoscope. As he must use his accommodation to see the image, he must be at a distance of 25 to $30 \mathrm{~cm}$. [This accommodative strain may be relieved and the image magnified by placing behind the ophthalmoscope a convex glass of $4 \mathrm{D}$, which adapts the emmetropic observer with relaxed accommodation for a point $25 \mathrm{~cm}$. distant.-ED.].

In high degrees of myopia the observer can in this way obtain an inverted image of the eye-ground without the aid of an additional lens, as may be seen in Fig. D 
(p. 27); for the rays emerging from a myopic eye converge to such an extent that they form a true inverted image at the far point. Hence, if on simply illuminating the eye and looking through the hole in the mirror an inverted image of the vessels or part of the optic nerve is clearly seen within the red glare of the pupil, we may conclude that we have to deal with a high degree of myopia. To determine whether the image of the eyeground seen in the pupil is an inverted or erect one, the observer notes whether the vessels in the pupil move in the same or in the contrary direction as he turns his head from side to side. This point must, however, be very carefully investigated before a diagnosis of myopia is made, as it is possible by simply throwing the light into the eye from a certain distance to see a partial erect image in the pupil. It occurs when the eve under examination has a high degree of hypermetropia and is due to the fact that the rays diverge after leaving the eye (as shown in Fig. B). But, owing to the great distance of the eye under examination, the visual field is very small, only a small portion of the eye-ground is seen, and the image in the pupil moves in the same direction as the observer's head.

With the aid of a convex lens it is possible to obtain an inverted image in any eye, that is to say, any eye may be made to appear like a myopic eye, but the distance of the inverted image from the anxiliary lens varies with the refractive conditions. If the eye under examiuation is emmetropic, the emerging rays of light are parallel and the inverted image is found in the focal plane of the auxiliary lens; in myopia the image will be nearer the lens (for instance, at $I_{y}$. in Fig. F), and in hypermetropia farther away than the focus (for instance, at $\dot{H}_{y}$. in Fig. F). Hence, if the observer does not alter his accommodation, he has to move his head back when he examines an eye with a high degree of hypermetropia. As a rule, however, it is better to accommodate. The distance of the auxiliary lens [often called the supplementary lens 
or object glass] from the plane of the pupil of the examined eye should be approximately equal to its focal distance. In this way the pupil and the iris disappear completely and nothing but an image of the eye-ground is seen in the entire domain of the convex lens. The larger the glass the wider the view of the eye-ground obtained; hence,

\section{The Size of the Visual Field ${ }^{1}$}

in the indirect method depends chiefly on the size of the auxiliary lens, and to some extent on the refraction of the eye under examination and the focal distance of the auxiliary lens. The higher the degree of myopia of the eye under examination, the greater the ophthalmoscopic field; the higher the degree of hypermetropia, the smaller the ophthalmoscopic field. The nearer the auxiliary lens is brought to the eye, that is the stronger the auxiliary lens, the greater the number of rays emerging from the eye it can receive and the greater, therefore, the visual field. Finally, as in the direct method, the size of the visual field depends on the size of the area illuminated by the ophthalmoscope. A greater portion of the eye-ground is illuminated in the indirect than in the direct method; the illumination is also more powerful, as a concave mirror is generally used in this method. The size of the pupil does not affect the size of the visual field so much in the indirect as in the direct method; in other words, the eyeground can be seen quite distinctly even when the pupil is very small, although, of course, a contracted pupil cuts off a great deal of the light and the image may, therefore, become very indistinct if the pupil is too small.

To sum up, it may be said that the indirect method has the following advantages over the direct method: 1 . Greater distance from the eye under examination, making the examination less irksome for both the patient and the surgeon ; 2. A larger visual field, so that a much greater

[ 1 "Gesichtsfeld" is the word used; in English text-books simply the word "field" is usually employed.-ED.] 
area of the fundus can be examined at one time; 3 . Correcting lenses on the ophthalmoscope can be dispensed with; 4 . The examination is possible even when the pupil is contracted. On the other hand, with the indirect method one sees the eye-ground under a lower magnifying power, and this is the principal difference between the two methods.

\section{Enlargement of the Image in the Direct and in the Indirect Method.}

The size of the image obtained by the direct method depends on several conditions: 1 . The refraction of both patient and surgeon; 2. The distance between the eyes of patient and surgeon; 3 . The distance between the correcting glass and the eye under examination. v. Helmholtz ${ }^{1}$ and, later, Mauthmer ${ }^{2}$ calculated the magnification for emmetropic eyes and found it to be one of $14 \frac{1}{3}$ diameters. L. Weiss ${ }^{3}$ obtained approximately the same result-15.6-by directly measuring a dead eye that had been carefully examined in this respect during life.

In hypermetropia the size of the image is smaller, in myopia larger, than in emmetropia, provided that the correcting lens is placed at a distance of $3 \mathrm{~cm}$. from the eye under examination. If the hypermetropia is due to abuormally weak refractive power, as, for instance, to aphakia, the image is smaller than in hypermetropia due to shortening of the axis, just as it is smaller in myopia due to the lengthening of the axis than in myopia due to abnormally strong refractive power of the refracting system (Mauthner, loc. cit., p. 185).

In hypermetropia the size of the image is smaller when the correcting lens is at a greater distance from the examirred eye, and, conversely, the image is larger in myopia

${ }^{1}$ v. Helmholtz, Handbuch der Physiologischen Optik, second edition, p. 217 .

${ }^{2}$ Mauthner, Lehrbuch der Ophthalmoskopie, p. 177.

3 L. Weiss, Arch. f. Ophthalmologie, vol. xxiii. 
when the lens is at a greater distance from the eye (Mauthner, loc. cit.).

In the indirect method the size of the image depends chiefly on the strength of the auxiliary lens; that is, the stronger the lens, the smaller the image and the greater the visual field. According to v. Helmholtz (loc. cit., p. 218), if we take Listing's schematic eye as a basis, we obtain a magnification of 3 diameters with an auxiliary lens of $22 \mathrm{D}$ (focal distance $45 \mathrm{~mm}$.), and a magnification of 4 diameters with a lens of $16.6 \mathrm{D}$ (focal distance, 60 $\mathrm{mm}$.). The refraction of the eye under examination also has some influence on the size of the image. In hypermetropia the magnification is somewhat greater, and, conversely, in myopia it is somewhat weaker. According to Mauthner (loc. cit., p. 230), the magnification is greater in hypermetropia due to diminution in the length of the axis than in hypermetropia due to diminished refractive power of the dioptric system; and, conversely, the magnification is less in myopia due to lengthening of the axis than in myopia due to abnormally strong refractive power of the dioptric system. Whereas, therefore, in the direct method the fundus appears appreciably modified, that is under 12-14-20 diameters, the magnification is only from 2-4-8 diameters in the indirect method with a convex lens of $17 \mathrm{D}$, which is the most suitable for this method.

On the other hand, the indirect method, as has been stated, offers the advantage of a larger visual field, hence it follows that to obtain a general idea of the conditions in as short a time as possible it is best to examine first by the indirect method, and if it is desired to make a more accurate study and to measure the refraction, an examination may be made by the direct method which shows the eye-ground under a greater magnification. [Students in this country are apt to neglect indirect oph thalmoscopy. This is unfortunate, and Haab's advice, to employ first the indirect and then the direct method, is sound and should be followed.-ED.] 
The refraction can also be measured by the indirect method. The method devised by Schmidt-Rimpler ${ }^{1}$ is the best, because the observer does not need to relax his accommodation, a feat which is often very difficult, especially for hypermetropic eyes. The position of the inverted image of the eye-ground, or, in other words, the distance of the image from the auxiliary lens, is found (ef. Fig. F) with the aid of a special apparatus. In this connection the reader should reeall what has been said on page 19. Rays emanating from a luminous point after being reflected by the eye return to that point provided the eve is placed in proper relation to the flame. If, therefore, the accommodation is relaxed, we can find the absolute refraction by means of such a luminous point. The eye is first rendered myopic by means of a strong convex lens placed in front of it, so that the emerging rays unite to form an inverted image in front of the eye. Thus, for example, in emmetropic eyes a lens of +10.0 brings the image $10 \mathrm{~cm}$. in front of the auxiliary lens. The souree of light used should not be an actual flame, but the inverted ophthalmoscopie image of a flame placed to one side of the person examined. The observer, looking through the hole in the ophthalmoscope, alternately carries the instrument backward and forward until the inverted image of the flame appears clearly outlined on the eyeground. When this point is reached the retina and the image of the flame correspond to conjugate foci, hence the image of the flame, the distance of which from the ophthalmoscope can at once be measured, coincides with the far point of the examined eye, providing the latter is looking into infinity, or, in other words, has relaxed its accommodation. By determining the far point of a myopic eye we, at the same time, determine its refraction. The accuracy of Schmidt-Rimpler's method depends on the absence of accommodation in the examined eye. This is not readily accomplished when the image of the flame is thrown on the macular region, or, in other words, when the examiner-as he ought to do-tries to measure the refraction of the macular region. If we do not paralyze the accommodation of the examined eye by means of a drug, we are forced to measure the refraction of the retina between the macula and the optic nerve.

The direct as well as the indirect method further enables us to determine irregularities in the surface of the eye-ground, and it is even possible to measure such inequalities by the direct method if the observer is skillful. It often happens that the head of the optic

1 Schmidt-Rimpler, Augenheilkunde und Ophthalmoskopie, sixth edition, p. 196. 
nerve is excavated, so that instead of a disk we have a more or less marked depression. The opposite condition, in which the head of the nerve projects into the eye, is equally important. And, finally, a neoplasm or a foreign body on the eye-ground may project into the vitreous body. If, then, the retina, for instance, appears emmetropic, the floor of the excavation, being further removed from the refractive system of the eye, will show myopia. If the retina is emmetropic and the optic nerve is swollen and projects into the globe, the summit of this prominence will show hypermetropia, because the distance from the lens at that point is diminished (cf. Fig. B, p. 27), or the retina about the optic nerve may show a hypermetropia of $5 \mathrm{D}$ and the floor of the excavation M. 5.0, in which case there is a difference of $10 \mathrm{D}$ between the two points, which would give a difference in level of $3.47 \mathrm{~mm}$. In the same way it might be possible to measure how far the swollen nerve-head or a neoplasm projects into the globe. For every diopter of difference in the refraction we calculate $0.34 \mathrm{~mm}$.

In the indirect method inequalities in the eye-ground are determined by moving the convex lens in front of the eye from side to side without, however, for a moment losing sight of the eye-ground or of the optic disk. If the nerve is excavated, the edge of the excavation moves in front of the floor in the same direction as the convex glass. If the head of the nerve projects, the summit of the prominence moves backward and forward with the auxiliary lens; in other words, a plane lying nearer the observer moves in front of a more remote plane in the same direction as the convex lens. This phenomenon is called parallactic displacement. One who is expert in examining in the erect image can take advantage of the process of parallactic or perspective displacement to determine inequalities of the eye-ground. The examiner approaches within a suitable distance of the eye to be examined, and, by moving his head to and fro, recognizes corresponding movements of objects, which occupy various planes. In 
this way very slight differences in level, such as a hole in the center of the retina, can be recognized in the eye-ground (Fig. 49).

There remain to be mentioned two methods of examination in which the observer also sits at a certain distance from the eye under examination, and both of which are also performed by means of a plane mirror, although a coneave mirror may be used.

\section{Examination by Transmitted Light.}

This procedure is very simple, but none the less inportant. It is also done with the ophthalmoseope, and should precede the methods described so far. By its use we are able to determine :

1. Opacities in the refiractive media-viz., the cornea, the aqueous humor, the lens, and the vitreous. By simply illuminating the eye with a concave or, better, a plane mirror and looking through the opening, opacities appear like dark shadows in the red pupillary reflex, because the rays of light as they return from the eye-ground are arrested by the opacities, just as all opaque objects, even when colored white, appear dark when seen in front of a luminous surface. There is no better way of detecting the whitish opacities produced by a gray cataract.

(a) In the corneal region this method reveals not only the presence of opacities, but also their position with reference to the pupillary region, which determines their influence on the visual acuity of the eorresponding eye. For, if the examiner requests the patient to look him straight in the eye, he can accurately recognize by transmitted light how much a corneal opacity diminished the transparency of the pupillary region, or, in other words, to what extent it impairs perfect vision. This point is often of inportance in damage cases.

By transmitted light the finer vessels in the cornea, which often make their appearance when this membrane is inflamer, can also be detected far better than by any other method, for they appear as dark lines contrasting sharply 
with the bright pupillary field. The procedure is particularly useful for detecting the broom-like vessels which persist after parenchymatous keratitis. These vessels, like the punctate deposits which will presently be described, are best seen with a magnifying loupe. For this purpose a convex lens, 8.0 to 16.0 , is placed behind the opening in the ophthalmoscope (by rotating the disk), and the distance from the eye to be examined which corresponds to the focal distance of this lens is found by advancing and retreating until the corneal vessels and other structures appear quite faint.

The same method may be adopted for studying the important deposits, which appear in iridocyclitis, usually on the posterior surface of the cornea, i.e., on the endothelium of Descemet's inembrane, especially in the lower quadrant. In studying the lower segment of the cornea the patient should be directed to glance slightly upward.

The adhesions of the iris to the lens (synechiæ), which are caused by iritis and project like villi into the pupillary region, are distinctly seen by transmitted light, because the latter is usually weaker than lateral illumination, so that the pupil is wider and the villi are more conspicnous.

(b) Opacities of the lens must be carefully scrutinized by transmitted light. It is always wrong to make a diagnosis of beginning gray cataract without an examination by transmitted light. In old persons, owing to the physiologic sclerotic changes of old age, the lens gives a fairly wellmarked gray reflex by daylight or under lateral illumination, and an inexperienced observer often makes the mistake of diagnosticating "gray cataract." In such cases examination by transmitted light at once shows that there are no genuine cataractous opacities, which most frequently radiate from the equator of the lens and project like a phalanx of spears or wedgres into the pupillary region. (See Atlas and Epitome of External Diseases of the Eye, third edition, Plate 38.)

Transmitted light is also very well adapted for exami- 
nation of an opacity at the posterior pole of the lens, socalled posterior polar cataract, which is difficult to see by direct light even when the pupil is widely dilated and the light is thrown straight into the eye. The opacity is usually circular or in the form of a rosette. The following points should be remembered in locating such an opacity : If with transmitted light and a moderately dilated pepil a dark opacity is seen within the bright pupillary field, while the patient looks straight before him, this opacity may be situated in the middle of the cornea, at the anterior pole of the lens or at the posterior pole (or possibly even in the nucleus). To determine the exact location of the opacity the patient is directed to glance slightly upward, downward, to the right, and to left. When the eye is rotated upward, a central corneal opacity will move upward in front of the pupil, and the examiner will be able to see into the eye underneath the opacity. But if there is an opacity at the anterior pole of the lens, as happens not so very infrequently, it will remain in the middle of the pupil, move upward with the pupil, and obstruct the view into the eye just as much as when the patient looks straight before him. If the opacity is situated at the posterior pole of the lens, the pupil will move upward in front of it, that is to say, the opacity apparently moves downward and the examiner sees into the eye over the top of the opacity. An opacity situated in the nucleus also descends somewhat when the eye is rotated downward.

Transmitted light offers the surest means of recognizing an opacity due to lamellar cataract. Since the examiner's object is to determine accurately the peripheral circular outline of the cataract, the pupil often has to be dilated with homatropin ${ }^{1}$ (or euphthalmin) and inspected while the patient glances to the right, to the left, or upward. $\mathrm{By}$ this method, better even than with lateral illumination, the peripheral border of the cataract, which is parallel to the equator of the lens and at a varible distance from it,

${ }^{1}$ Atropin should never be used in dilating the pupil merely for purposes of examination. 
can be accurately determined. (See Atlas and Epitome of Externally Visible Diseases of the Eye, Plate 38.)

(c) Transmitted light is especially well adapted for the detection of opacities in the vitreous, which are also exceedingly important. Whereas fixed opacities in the refractive media move with these media when the eye is rotated in various directions, freely movable opacities in the vitreous continue to move after the eye has come to rest. It is at this instance that the examiner must carefully look for any shadows that may be moving in the bright pupillary field. Such shadows are produced by shreds of tissue, particles of exudate, membranes, and the like in the vitreous. Diffuse opacity of the vitreous cannot be determined by this method, and its existence can only be inferred when the eye-ground in the inverted or ereet image is veiled, and lenticular or corneal opacity can be excluded from the etiology.

Foreign bodies in the vitreous are dotected in the same manner by transmitted light. But in the case of foreign bodies the patient must be instructed to move the eye very rapidly, and in that way a particle of iron or copper lying immovable and, therefore, invisible on the floor of the vitreous may be tossed up and rendered visible, even if only for an instant. Rotation of the eye in various directions must be kept up for some time, until finally the foreign body is tossed up from the bottom, or in from the side, and becomes visible.

Examination with transmitted light is also useful as a preliminary method in the detection of a foreign body that is fixed somewhere in the eye-ground, and may be usually recognized by its somewhat paler color. As soon as the examiner's line of rision strikes the foreign body lying in or upon the retina the metallic surface throws back a reflex which illuminates the pupil, unless the body is covered with blood or exudates. If the pupil under illumination appears white, instead of red or grayish red, the convex lens is brought into use, and the foreign body appears in the inverted image. 
2. If the eye is examined under simple direct illumination, marked errors of refraction, either myopia or hypermetropia, can be detected much more quickly and more easily than by the mensuration methods already deseribed, excepting as to the exact number of diopters. For it follows from what was stated on pages 41 and 42 that, at the distance which is maintained in examining in the inverted image, structural details of the eye-ground (blood-vessels and the like) can be seen in the illuminated pupil, in the inverted image in cases of high myopia, and in the erect image in cases of marked hypermetropia.

High degrees of myopia ean even be measured after a fashion by this method. Thus, the examiner gives himself an artificial myopia of, say, 10 D. by using a suitable convex lens. If he is emmetropic he holds a $+10 \mathrm{D}$. lens behind the ophthalmosenpe; if he has a myopia of 1 to 10 D. he will need a weaker plus lens, one that will bring his myopia up to 10 (thus with a myopia of 3.0 he will only need a +7.0 lens). If the examiner is hypermetropic he must add his total hypermetropia to the $10 \mathrm{D}$. Then, without accommolating, the examiner causes the image of the myopic eye-ground, which appears by transmitted light to coincide with his far point by retreating from the latter as far as possible without making the image appear blurred, he then measures the distance between his own eve and that of the patient. Suppose this distance is $15 \mathrm{~cm}$; then the inverted image of the eye-ground lies $10 \mathrm{~cm}$. in front of the examiner's and $5 \mathrm{~cm}$. in front of the patient's eye. Hence, the patient's far point is at a clistance of 5 $\mathrm{cm}$. or, in other words, he has a myopia of $20 \mathrm{D} .\left(1 \frac{n}{5} 0=20\right)$. Needless to say, the patient must not aecommodate during the examination. The inverted image of the eye-ground sometimes coincides with the patient's as well as with the examiner's far point ; but the latter artificially changes the distance to $10 \mathrm{~cm}$., so that the ealculation can be based on this distance. If the oculist has no ophthalmoseope with strong concave lenses and does not care to undertake the shadow test (which will be discussed presently), he 
can determine a high myopia in his patient's eye by this method.

Keratoconus shows itself by transmitted light, especially if the ophthalmoscopic mirror is moved to and fro, or the mirror reflex in the pupil is carried around in a circle. A circular shadow is seen in the bright pupillary field. The same oceurs when the lens is conical posteriorly (lenticonus posterior) or, what is more frequent, the refractive index of the nucleus is greater than that of the cortex. This condition may develop during old age and cause considerable disturbance of vision.

In certain cases of beginning cataract valuable information may be obtained by examining with transmitted light, especially if the above-mentioned genuine opacities have not yet developed, and vision is interfered with simply by irregular refraction in the lens. On rotating the ophthalmoscope delicate shadows make their appearance in the bright pupillary field.

3. Transmitted light is also to be recommended in examining the interior of the eye for the presence of tumors, retinal separation, circumscribed exudates, and the like, occupying the anterior half of the vitreous. The examiner can get his bearing more quiekly and more easily by this method than by any other in cases of this kind. A sarcoma of the choroid appears as a dark mass, a glioma appears bright, abscess causes bulging into the vitreous, and the same picture is produced when a large retinal separation of some standing, and therefore opaque, causes single or multiple prominences in the anterior portion of the vitreous. Strictly speaking, we are dealing with an erect image in these cases. The vessels belonging to the retina, which have become separated by the sarcoma or in some other way, are seen directly, as are also the hemorrhages and dilated vessels, which are so frequently present on the surface of a glioma.

So long as a large retinal separation remains transparent it is most surely detected by transmitted light. For example, if a retinal separation of this kind is situated on 
the nasal side, the beautiful pink brightness of the pupil will be changed to a peculiar grayish red when the eye is illuminated from the nasal side. Secondly, retinal vessels will be seen in the altered pupillary field in the erect image, recognized by the fact that, when the observer moves his head, they move in the same direction. This local hypermetropia of the retina can only be due to local diminution of the distance between the retina and the refractive system of the eye, and, therefore, indicates that the membrane is displaced or, in other words, that there is a retinal separation.

\section{THE SHADOW=TEST, OR SKIASCOPY.}

The diseoverer, Cuignet, called the methorl lierutoscopy; it is also known as pupilloscopy and retinoscopy. This method of examination is also best performed with the plane mirror, and offers the easiest means of determining the refraction. Retinoseopy is the method recommended for any one who is unable to determine refraction, and especially astigmatism, by the direet method in the manner described, if he does not care to use Schmidt-Rimpler's apparatus. It also requires practice, however, and is not without ineonveniences. In retinoscopy, as in SchmidtRimpler's method, the object is to determine the position of the far point of the eyc [point of reversal], sometimes with the aid of a convex lens, so as to bring the far point to a convenient distance, that is, between the observer and the patient. This is especially important in low degrees of myopia and in hypermetropia. The far point should, if possible, be brought to a distance of 20 to $40 \mathrm{~cm}$. To determine the optical conditions of the eye under examination by this method, we utilize instead of an image of the eye-ground the movement of the illuminater portion of the eye-ground as the retinoscope is moved from side to side. The retinoscope illuminates a certain portion of the eye-ground, and by looking through the opening the observer sees a corresponding 
movement of the illuminated portion of the eye under examination.

Now it is to be remembered that when the light from a flame placed to one side of the patient is thrown into the eye by means of a plane mirror, the illuminated portion of the eye-ground moves from above downward if the mirror is turned from above downward, or it moves from right to left if the mirror is turned from right to left. By turning the mirror from right to left we mean turning it as if we wished to throw light into the right and then into the left eye of the person examined.

The illuminated portion of the eye-ground of the patient, therefore, moves in the same direction as that in which the plane mirror is rotated, or, as one may say, "with" the turning of the mirror.

If, however, the eye of the observer is beyond the far point of the eye under examination, the illuminated field or the shadow which bounds it in the pupil does not move in the same direction as the illuminated portion of the eve-ground. This is the case in high degrees of myopia, because the rays emerging from the eye under examination converge and cross each other at the far point, thus causing the shadow to move in the contrary direction. Hence in myopia the shadow in the pupil moves in a direction contrary to that of the mirror; that is to say, if the mirror is turned to the left, the shadow moves to the right. To do this the degree of myopia must be such that the distance of the far point is less than the distance of the ophthalmoscope from the eye under examination. If, for instance, the distance between the mirror and the eyc under examination is $50 \mathrm{~cm}$., and the shadow moves in the opposite direction, there must be a myopia of more than $2 \mathrm{D}\left(\frac{100}{50}=2.0\right)$.

Conversely, the illuminated field in the pupil moves in the same direction as the mirror when the examiner's eye is within the far point of the eye under examination; that is to say, in a low degree of myopia or emmetropia, or when the far point lies behind the eye under examina- 
tion; in other words, in hypermetropia. Under these conditions the illuminated field moves to the left, for instance, when the mirror is turned to the left.

On the other hand, if the position of the observer's pupil corresponds exactly with the far point of the eye under examination, the illuminated portion of the pupil will move in neither direetion and the pupil will simply appear alternately dark and bright. Whenever this phenomenon is distinetly seen as the observer alternately advances and recedes from the patient, the distance between the two eres is to be carefully measured with the tape-measure. 'The result corresponds to the distance of the far point, and thus gives the refraction, provided that the person examined has relaxed his aeeommodation. If, for instance, movement disappears at a distance of $20 \mathrm{~cm}$. between the observer's eye and that of the patient, the latter has a myopia of $5.0 \mathrm{D}\left(\frac{100}{50}=5.0\right)$.

If light and shadow in the pupil move in the same direction, the observer is within the far point, and he must then recede until the movement disappears as he turns the mirror from sirle to side, or he may place a convex lens in front of the patient's eye so as to bring the far point within a eonvenient measuring distance, as explained above; that is, to about 30 or $40 \mathrm{~cm}$.

In choosing this anxiliary lens the following is to be borne in mind: If the illuminated field in the pupil moves in the same direction and quickly, and the concavity of the border is flat, one has to deal with a low myopia, an emmetropia, or a low hypermetropia. In such a case a weak convex lens of about +3 should be selected. If the illuminated field in the pupil moves slowly and the border is strongly concave, one has to deal with a high degree of hypermetropia. A strong convex lens is then selected, which must be allowed for in the ealculation. If, for instance, with a lens of +7 , the far point is found at $33 \mathrm{~cm}$, one has to deal, not with a myopia of $3.0\left(\frac{100}{33}=3\right)$, but with an hypermetropia of 4.0 , because it is evident that the myopia of 3.0 is an 
artificial myopia produced by the 3 diopters of the correcting lens, and the remainder of 4 diopters would remove the far point of the eye under examination to infinity and thus correct its error.

The shadow-test can also be carried out with the ordinary concave mirror, but in that case the movements of the shadow in the pupil are exactly reversed; that is to say, the shadow moves in the same direction when the observer is beyond the point of fixation, and in the contrary direction when he is within that point, or when there is hypermetropia. The movement of the shadow may be conveniently observed at a distance of 1 meter and $20 \mathrm{~cm}$. from the patient. If the shadow moves in the same direction as the nirror, there is myopia of one or more diopters. The eye is then corrected with successively stronger concave lenses until one is found with which the movement of the shadow becomes reversed. Such a glass removes the far point of the eye under examination to beyond 1 meter (1 diopter), and the last lens $(n \mathrm{D})$, with which the shadow still moves in the same direction as the mirror, corrects it nearly to $1 \mathrm{D}$. The myopia is, therefore, $n \mathrm{D}+1 \mathrm{D}$.

If with a concave mirror the shadow moves in the contrary direction, the patient either has a myopia less than $1 \mathrm{D}$ or he is emmetropic or hypermetropic. In such a case the eye is corrected with successively stronger convex lenses until the shadow moves in the same direction as the mirror. If such a glass is $n \mathrm{D}$, the patient's refraction is $n \mathrm{D}-1 \mathrm{D}$.

The shadow-test also has its drawbacks. It requires for its sucecss that the puril be widely dilated-if necessary, by artificial means. Even with a widely dilated pupil it is not always easy to tell when the movement of the illuminated field ceases $\mathrm{c}_{\mathrm{L}} \cdot$ becomes reversed, nor is it always easy to get the patient to relax his accommodation completely, as the surgeon's manipulations excite his attention and cause him to fix some object near him. Finally, the refraction obtained, instead of being that of 
the macula, is often only that of a neighboring portion of the eye-ground. The method will be found in detail in Neustätter's Grundriss der Theorie und Praxis der Schattenprobe (Skiaskinpie) nebst Tafeln und Phantomen zur Skiaskopie, München, J. F. Lehmann, 1900. [For American students the most useful works on the shadowtest have been published by Edward Jackson and James P. Thorington.-ED.]

[For the sake of convenience the method of application of the shadow-test in the determination of regular astigmatism is inserted. Dr. Jackson's description is given: "The principles involved and the methods to be employed are essentially the same as in myopia or hypermetropia, but the refraction has to be determined in the principal meridians instead of in any meridian indifferently, as it can be where all meridians are alike. To deternine the refiaction in a certain meridian the light must be made to move back and forth in that particular meridian by rotating the mirror about an axis at right angles to it.

"The direction of either of these two principal meridians is revealed by the area of light in the pupil assuming the form of a more or less distinet band of light, extending across the pupil in the direction of this meridian when its point of reversal is approached. For the higher degrees of astigmatism this band is very noticeable, and fixes with the greatest accuracy the direction of the principal meridian. When the band-like appearance is most noticeable it is easy to cause its apparent movenent from side to side, but it is more difficult to distinguish the movement in the direction of the length of the band. Sill, this latter movement is one that is to be especially watched and its reversal-point determined.

"When the amount of astigmatism is very low the appearance of a band may be very indistinct, or not at all perceptible; but in such cases it is found that when we have reached the point of reversal for movement of the light in one direction there is still distinct movement, either direct or inverted, in the direction at right angles to this, and we thus know we have tested one meridian of an astigmatism, and must in the same way ascertain the point of reversal for the other at right angles to it. When the surgeon is closer to the eye than the point of reversal for either meridian, the movement will be with the light on the face in all directions. When he is at the point of reversal for the meridian which has its point nearer to the eye than the other meridian there will be no distinguishable movement in this direction, but still a direct movement at right angles to it. When he is between the two points of reversal there will, in the direction of the nearer meridian. be an inverted movement of the light, but in the other merid- 
ian a direct movement. When the farther point of reversal is reached the direct movement in its meridian ceases, while the movement in the other meridian continues inverted. When the surgeon has drawn back beyond both points of reversal, the movement is reversed in all directions.

"Having determined the amount of myopia, natural or artificial, in both principal meridians, the strength of the cylinder reguired to correct the astigmatism will, of course, be the difference between the refraction for the two meridians. Having thus ascertained it, it is well to put this cylinder before the eye and to see if it does accurately correct the astigmatism, giving the same point of reversal for all meridians of the cornea; and, for accuracy, the spherical lens which will bring this point of reversal to the distance of one or two meters should be used with it."-ED.]

\section{CHOICE OF AN OPHTHALMOSCOPE.}

Before proceeding with a description of ophthalmoscopic technic, it will be well to devote a few pages to the discussion of the instrument itself. I shall not attempt, however, to describe all the ophthalmoscopes at present in use, for that alone would require a book. Mere description withont criticism would be of little practical value, and to compare all the various forms of ophthalmoscopes and test their practical usefulness would be a Herculean task. I have, it is true, used a good many of the best and most popular instruments at present in use, and should be in a position to criticise them, but as I am not acquainted with all of them I might be led to do an injustice to some. I shall permit myself to mention only one instrument. It is a small simple refraction-ophthalmoscope introduced by me ten years ago, at least among my pupils, in place of Liebreich's ophthalmoscope, which, on account of its many defects, has always been to me a thorn in the flesh. Recently simpler ophthalmoscopes than Liebreich's, for examining in the inverted image only, have been placed on the market ; but in my opinion an ophthalmoscope for students should also permit examination in the erect image, or the direct methor.

It will be enough if I lay down the principles on which 
an ophthalmoscope should be constructed to fulfil all the requirements. These principles are the same for all instruments and, as in the case of the microscope, they are extremely simple. As in the construction of the latter instrument, the only factors that vary are: 1 . The mechanical portion; 2. The quality of the material and workmanship.

In my experience most of the larger instruments in ordinary use - the so-called refraction-oph thalmoscopesin the main show careful workmanship, especially those that are turned out by well-known makers of optical instruments in various countries. It is always well, however, to test one's instrument in regard to the grinding of the mirror and lesses, the proper enumeration of the lenses, etc., and to refuse it if it is in any way defective. As regards the mechanism, the instrument may be constructed according to the riews and personal preferences of the author or of the purchaser. The important point is that the instrument should work properly and be easy to manipulate. The proper construction in this respect can easily be deduced from what has been said above in explaining the ophthalmoscope. When we come to consider the mirror itself, I find from my own experience that it is well to have both a concave and a plane mirror, or at least a concave mirror. A plane mirror may, if necessary, be dispensed with, or, if the instrument does not possess one, it may be supplied by taking a suitable piece from any good mirror and scraping away the coating from a small spot in the center if, for instance, it is desired to make a careful examination for flocculi in the vitreous, or to employ the shadow-test, although the latter can also be performed with a concave mirror. The best concave mirror, in my opinion, is one which has a focal distance of 14 to $17 \mathrm{~cm}$. This will do equally well for the direct and for the indirect method. In testing the focal distance of the mirror one should notice at the same time whether the inage it reflects is sharply defined, as, for instance, a lamp placed at a distance, so 
as to determine whether the glass is carefully ground. An important point is the size of the opening. It must not be too small or it will not admit enough light into the observer's eye. This is especially important in the refraction-ophthalmoscope, because, on account of the necessity of interposing auxiliary lenses and tilting the mirror, the observer is often forced to work at some distance from his patient. The diameter should be at least $3 \mathrm{~mm}$. on the anterior, and a little greater on the posterior surface, so that the opening is slightly larger behind than in front. It is particularly important that the walls of this short canal be well blackened. It must not produce any reflexes whatever, because they are exceedingly disturbing, especially in examining in the erect image. Even the largest optical firms are not particular enough in this respect, especially in the case of cheap ophthalmoscopes.

In all the larger. instruments designed for the measurement of refraction, it is absolutely indispensable that the mirror be capable of being tilted if the measurements are to be exact; for if it is desired to throw the light from a lamp placed to one side of the patient into the latter's eye, the mirror must be turned slightly toward the lamp, as will be seen in Fig. A t that is to say, the plane of the mirror as seen from above will form an angle with the plane of the patient's face. The consequence is that the observer looks obliquely through the lenses placed behind the mirror if they are closely applied to the mirror; but if we look obliquely through a spherical lens, the latter acts like a cylinder, that is, the rays are refracted more in one direction than they would be if they passed through perpendicularly. Besides, if the correcting lens is closely applied to the mirror, the observer is farther away from the correcting lens than he should be. Hence, the newer ophthalmoscopes are so arranged that the mirror can be set at an angle to the plate which holds the correcting lenses, either to one side or the other, so that the lamp can be placed either to the right or to the left of the patient. 
With the indirect method there is no necessity of tilting the mirror, as correcting glasses are not, as a rule, required. Hence, those instruments which are provided with two mirrors, one for the indirect and one for the direct method, are usually so arranged that only the mirror for the direct method can be tilted. But as it is sometimes desirable to use correcting lenses in the indirect method for the purpose, for instance, of obtaining an enlarged image, it is best, on the whole, to have a concave mirror of $16 \mathrm{~cm}$. focal distance suitable for both methods placed at an angle, and to use a small plane mirror for the examination of flocculi in the vitreous and for the shadow-test. This plane mirror need not necessarily be fastened to the ophthalmoscope.

This brings us to the mechanical portion of the instrument, and we first have to consider the most important point, namely, the attachments for the correcting lenses behind the opening in the mirror. The correcting lenses should be as near the mirror as possible, nor should too many be placed one above the other, as that would diminish the amount of light in the image. If one lens is placed over another, the two should be in close contact, so that the observer's eye may be as close as possible to the opening in the mirror.

The correcting lenses are now usually arranged along the edge of a round disk, which can be rotated behind the mirror in such a way that the various lenses are brought exactly behind the opening in the mirror, and the center of the lens always corresponds exactly with the center of the opening. This is accomplished by means of catches. In many ophthalmoscopes two disks of this kind are placed one above the other, so that the observer always looks through both. In others the disks are shanged according to the lenses required, which is very troublesome. I find that for ordinary purposes I get along very well with a single disk supplemented by a quadrant rotated on the same axis and containing lenses of very high power. The quadrant when not in use can 
be turned down toward the handle, as, for instance, in the instrument designed by Loring. If, for example, the disk contains 15 lenses: Concave 1 to 8 and convex 1 to 7 , and an empty hole, and the quadrant contains concave 16 and convex 16, and, in addition, concave 32 and 0.5 ,
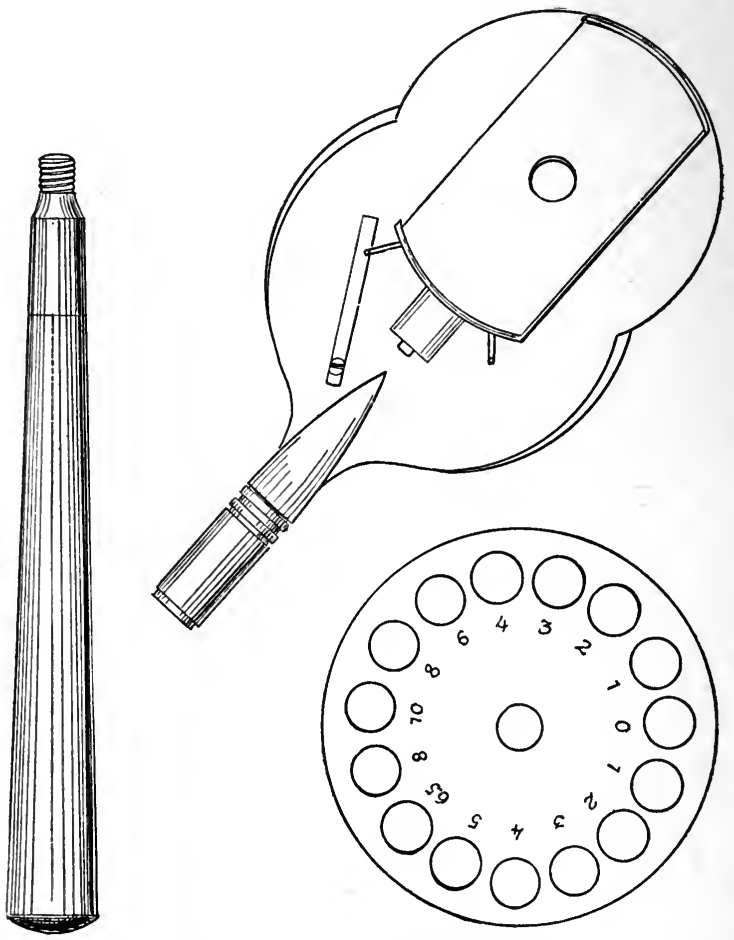

Fig. G.-Author's ophthalmoscope.

the combination of the disk and the quadrant places at our disposal: Concave 1 to 40 and convex 1 to 23, a total of 63 numbers, and, in addition, the half diopters from 1 to 8 concave. If, instead of the quadrant, the instrument is provided with an additional disk, the num- 
ber of lenses at the surgeon's disposal is considerably increased.

As the convex lenses must not be too small, their num-
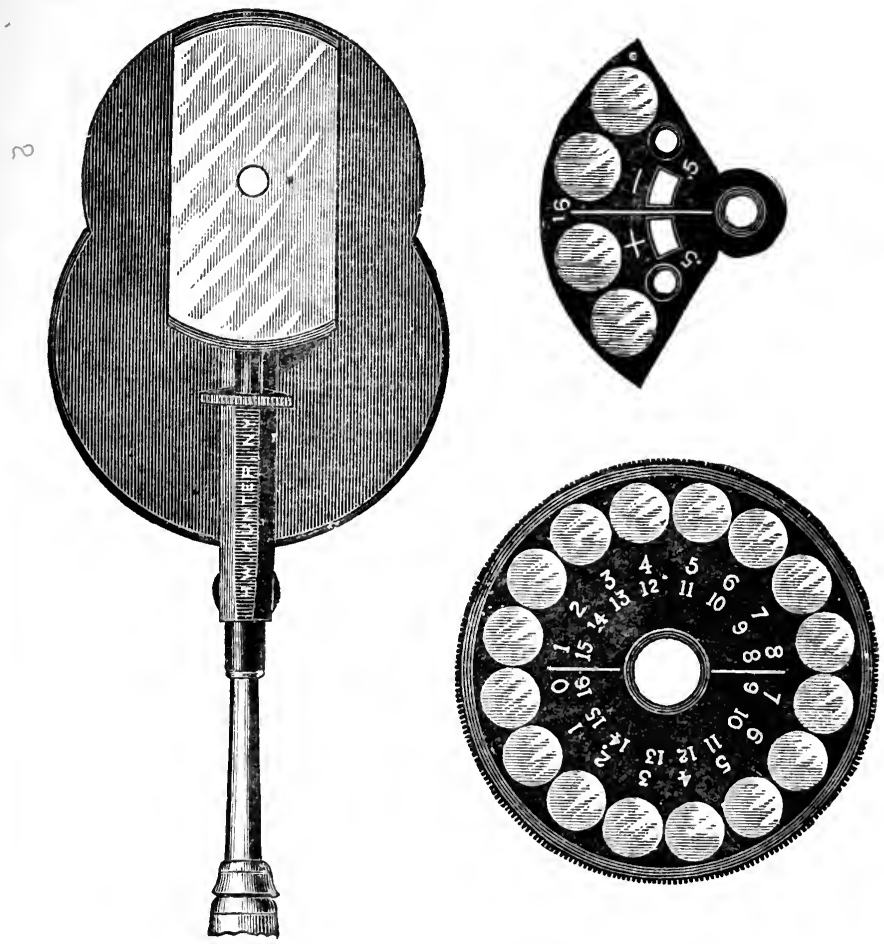

Fig. H.-Loring's ophthalmoscope, with tilting mirror, complete disk of lenses from -1 to -8 and 0 to +7 , and supplemental quadrant containing \pm 0.5 and \pm 16 D. This affords 66 glasses or combinations from +23 to $-24 \mathrm{D}$.

ber in the disk is necessarily limited. The diameter should not be less than $5 \mathrm{~mm}$.

Finally, a word in regard to the convex lens used to produce the inverted image. Much too little attention 
is paid to this auxiliary lens in many ophthalmoscopes. As the size of the case is usually limited, it is much
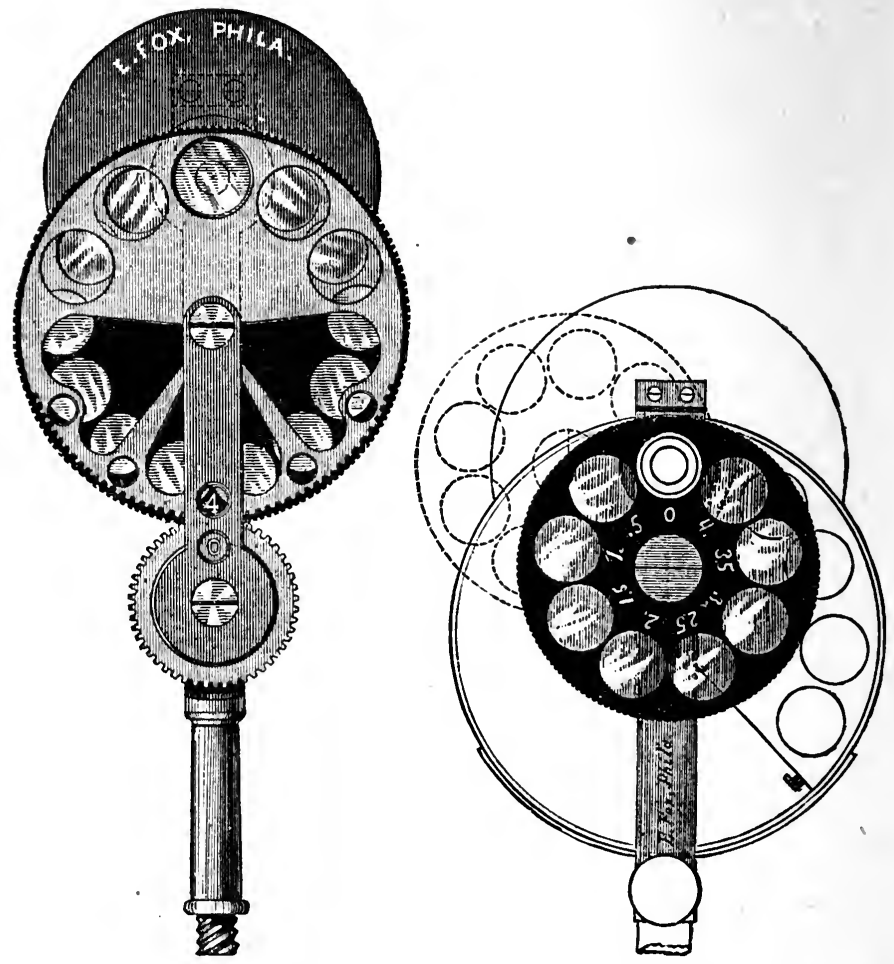

Fig. I.-Randall's modified Loring ophthalmoscope, in which the "quadrant" is moved by the cog below, so that every glass can be brought to the sight-hole without removing the instrument from the eye. A disk of concave cylinders 0.5 to 4 is excentrically mounted, so that each can be brought at any desired inclination of its axis into combination with any spherical. It gives 51 spherical lenses or combinations. The mirror can be detached to substitute a weak light, plane, or more concave mirror, or left off, uncovering the $6 \mathrm{~mm}$. breadth of the lenses when the instrument is used as an optometer.

better to have one lens of the proper size than two small ones, especially for the beginner, as it is important that 

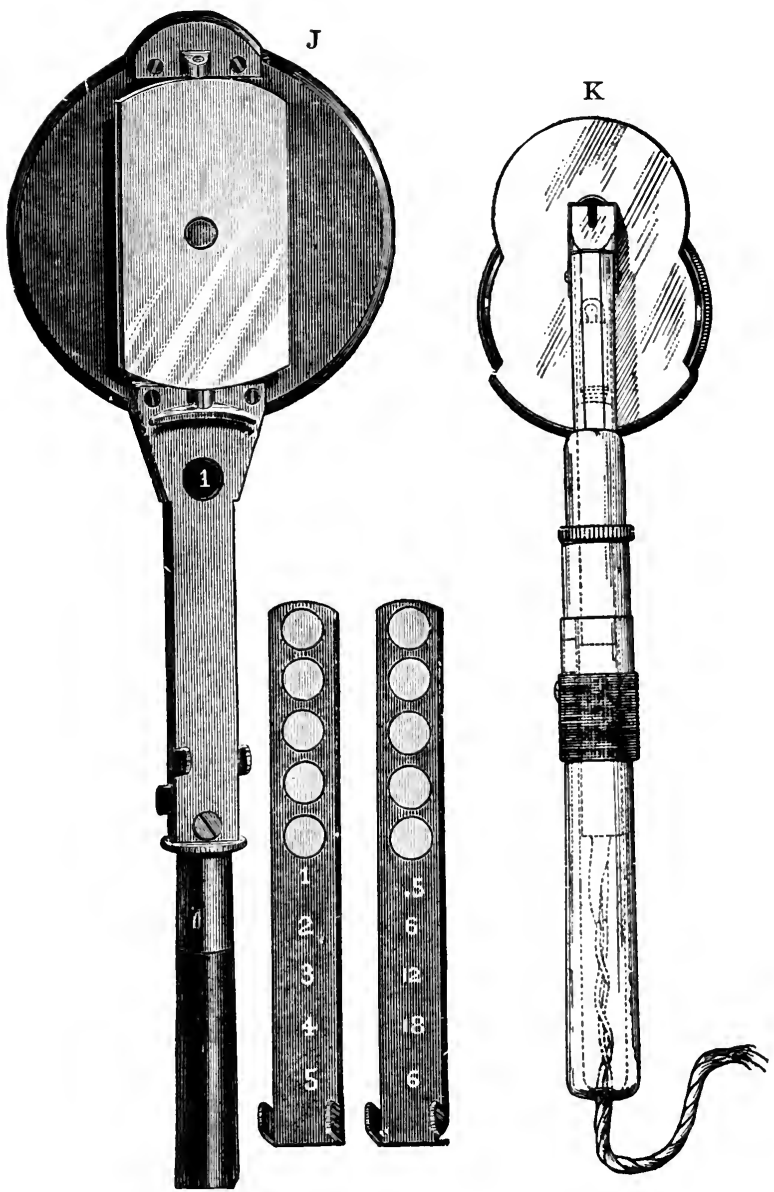

FIG. J-Jackson's ophthalmoscope, with two superposed slides of lenses coming singly or conbined behind the sight-hole of the tilting mirror. It gives 35 lenses or combinations, from +11 to $-18 \mathrm{D}$, with great convenience, and is exceedingly simple and thin.

Like most other ophthalmoscopes, the figures are red to indicate concave glasses, and white to mark convex, making mistake or confusion as to combinations unlikely.

Fig. K.-Marple's electric ophthalmoscope. 
the field should be as large as possible. For beginners it is best always to use the same lens, which should have a strength of about $17 \mathrm{D}$ or a focal distance of $6 \mathrm{~cm}$.; the size of the lens should not be less than $3.5 \mathrm{~cm}$. The best size is from 3.5 to $4 \mathrm{~cm}$. 'The mounting of the lens should be sufficiently wide to prevent its being scratched when laid on the table or soiled by the fingers when in use. For reasons which will be given later it is also advisable to have a short thick handle attached to the rim. If other convex lenses are needed in the indirect method for the purpose of obtaining a greater magnification, a +13 or +14 lens from the lens-case may be called into requisition. With a refraction-ophthalmoscope the inverted image can easily be magnified by bringing a convex lens of 2 to $4 \mathrm{D}$ behind the opening in the mirror and at the same time coming closer to the patient.

The only instrument I should advise anyone to buy is one that can be used for the direct method; that is, one with a considerable number of correcting lenses so attached that they can be rapidly brought behind the opening in the mirror, as, for instance, by means of the disk just described. In Liebreich's instrument the lenses are placed in separate holders. This is extremely troublesome and the lenses are nearly always soiled. It should also be possible to change the number of correcting lenses behind the mirror without removing the mirror from the eye. For ordinary work a small number of lenses, not greater than twelve, will be found sufficient. Hence, the disk need not be very great and the price of the instrument not unreasonable. Years ago I had a small ophthalmoscope of this kind made by $R$. Doerffel in Berlin. It has a movable concave mirror, a disk with fourteen lenses, and the proper kind of auxiliary lens. That they are strong is shown by the fact that a number of them have been bought and used by my pupils in the last ten years, and the price is so moderate (25 marks) that one feels justified in advising every student to buy one (Fig. G). I can also recommend it to the practising physician 
who does not eare to buy a large or expensive instrument, as it will be quite adequate to all his needs. [Among the many ophthalmoscopes at the student's disposal, in the editor's opinion, none is better than the Loring ophthalmoseope; Jackson's ophthalmoscope is also excellent. Randall's cylindrical lenses-attachment has been referred to ; vide diagrams $\mathrm{H}, \mathrm{I}$, and J.-ED.]

\section{THE METHOD OF CONDUCTING AN OPHTHALMOSCOPIC EXAMINATION.}

The ophthalmoscopic examination is best made in a dark room with a moderately large gas- or oil-lamp. An incandescent light, on account of the small size of the flame, cannot be used without a special appliance. Ophthalmoscope lamps with electric lights are made and recommended by Deus, Eversbusch, and others. Eversbusch's light can also be used for gas.

H. Wolff has constructed an ophthalmoscope (obtainable from Doerffel \& Färber, Berlin, Friedrichstr, 105a) which is filled with a small incandescent lamp. The ophthalmoscopic visual field (in the erect image) is very large with this instrument, and at the same time the retinal reflexes are very distinctly seen. Similar electric ophthalmoscopes have been constructed in England and America. Marple, of New York, commends such an instrument combincd with Morton's well-known and excellent ophthalmoscope, which can be obtained from E. B. Meyrowitz, New York and Paris (3 Rue Seribe). [In the opinion of the editor, Marple's electric ophthalmoscope (Fig. $K$ ) is the most useful instrument of this character at present available. The De Zeng instrument is also satisfactory. Electric ophthalmoscopes are essential for accurate bediside work, and most convenient on all occasions. They are particularly valuáble for daily observations of the fundus after decompressing trephining and other operations conducted in the course of brain surgerv._-ED.]

If an Aner incandescent gas-lamp is used, it is to be borne in mind that the light is more greenish white, 
which gives the eye-ground a different color as compared with the yellow light. The same applies to examination by daylight, which is allowed to enter the dark room through a suitable opening, which, however, may be recommended for certain cases in which it is important to recognize the natural coloration of a structure in the eyeground. With concentrated daylight it is possible to recognize the much-disputed vellow coloration of the macula lutea of the retina (Dimmer).

The patient should be placed at a table, at a distance of 40 to $60 \mathrm{~cm}$. from the observer, who sits opposite, with the table on his right hand. The table supports the lamp, which should, if possible, be on a level with the patient's eye. The patient should sit on a revolving stool, and the lamp should be so arranged that it can be raised and lowered at will.

The surgeon should make it a rule always to examine by lateral illumination before proceeding with the ophthalmoscopic examination until the habit becomes, as it were, second nature. This method brings out many changes that have an important bearing on the ophthalmoscopic examination and should, therefore, never be neglected. Thus, a beginner may waste a good deal of time in trying to obtain a clear image of the eye-ground by the direct method, because he has neglected to examine by lateral illumination and has, therefore, failed to discover opacities in the cornea or in the lens. The indirect method may be fairly successful even when the refracting media are somewhat opaque, because the light is more intense and the image is not so greatly magnified, but not the erect image. The surgeon then begins by placing the lamp to his right hand, takes up the convex lens that accompanies the ophthalmoscope, and allows the light (from the lamp) to pass through it, holding the lens at an angle and to one side, so as to throw a strong light into the anterior portion of the eye. By successively directing the luminous apex of the cone of light passing through the lens on the individual parts of the eye, he ex- 
amines them separately, if necessary, with the additional aid of a good magnifying glass. A binocular loupe such as Berger's is exceedingly useful. With it iris-nodules and other complex objects in the anterior chamber can be recognized at a glance. For a more detailed study the larger loupe designed by Westien and particularly that of Ziess, which supplies electric illumination of the object at the same time, are to be recommended.

After this inspection by lateral illumination, the lamp is placed by the side of and a little behind the patient, so that the light does not fall directly into his eyes, but remains in shadow as much as possible, and the surgeon proceeds with the ophthalmoscopic examination. The lamp should be as near the patient's head as possible.

The second step in the examination consists in a simple illumination of the eye. This excomination by transmitted light, in which we simply throw the light into the patient's eye with the concave mirror so that the pupil appears red, reveals, as stated above, any opacity of the cornea or of the lens and vitreous. In order to see any abnormalities near the periphery of the lens or ritreous, the eye must be examined in various positions, hence the patient should be told to look up and down and to either side, the surgeon keeping the light reflex on the pupil so that it constantly appears red.

The third step in the examination consists in the employment of the indirect method, because it affords a better general view of the entire eye-ground. For this purpose the convex lens of $17 \mathrm{D}$ is held in front of the patient's eye at a distance of $6 \mathrm{~cm}$., corresponding to its focal distance, and if the glass is provided with a handle, the remaining fingers of the hand that holds the lens will not obscure the patient's face and interfere with the sight of the other eye. The handle, therefore, has a distinct advantage, because the other eye must be made to maintain a certain direction by fixing an object at a distance. Thus, while examining the left eye, the patient is asked to fix the surgeon's ear, and to do this his right eye must not 
be covered. If the light is now thrown into the eye through the lens, and the observer looks through the opening in the mirror and the anxiliary lens, he will, if the anxiliary lens and mirror are in the proper position, immediately see the optic nerve [of the left eye]. During the examination the surgeon must accommodate for that point between the auxiliary lens and the mirror which corresponds to the position of the image of the eye-ground, as explained above. This is one of the difficulties of this method. The beginner usually accommodates either for the eye or for the auxiliary lens and gets an imperfect view of the image, since the latter is projected between his own eye and the auxiliary lens.

To overcome this defect it is advisable to practise with the same auxiliary lens on reduced inverted images of colored eye-grounds, such, for instance, as are found in the present volume. The picture should be held at a distance of $60 \mathrm{~cm}$. and examined with the auxiliary lens of $17 \mathrm{D}$ held at a distance of about $20 \mathrm{~cm}$. from the picture. Incidentally the student will learn to orient himself in the inverted image. By practising in this way he will learn to know and overcome another defect ineident to the indirect method, namely, the reflexes of the auxiliary lens. He will soon learn to look between them by holding the lens a little obliquely. After he has learned to deal with these reflexes it will be easier for him to overcome or disregard the corneal reflex, which at first is such a disturbing factor in the examination.

As soon as he has mastered the art of obtaining the inverted image in the actual subject, he must learn to keep the patient's other eye under observation by indirect vision, so as to control its direction. Children often disobey the order to look in a certain direction. Thus, they often will not look at the surgeon's left ear, when he wishes to inspect the left optic nerve, etc. To avoid wasting time, the surgeon must insist that the patient keep his eye in the same direction for some time. But after asking the patient to fix his left ear, the surgeon 
must be careful not to make it impossible by covering the patient's right eye with the hand that holds the anxiliary lens, as has been explained above. He must rest his hand on the patient's face in such a way as not to obstruct the other eye and make it diffienlt for the patient to fix the object he is asked to look at. If the anxiliary lens is supplied with a handle (as in the lens which goes with Haab's ophthalmoseope) it is very much easier to manipulate.

The surgeon must further learn to raise the upper evelid with one of the fingers of the hand that holds the convex lens. This may be necessary in examining the eye-ground while the patient is looking down, or in examining patients lying in bed. It is best done by laying the ulnar side of the tip of the fourth finger lightly against the upper lid and drawing it upward. The surgeon should never be content with an examination of the optic nerve and its immediate neighborhood, but should train himself to inspeet the peripheral portions of the eye-ground as well, and for this purpose should ask the patient to look up and down and to either side.

To obtain a view of the optic nerve of the right eye the patient is asked to look past the surgeon's right ear at the wall, as the optic nerve lies about $1 \tilde{5}$ degrees to the nasal side of the posterior pole of the eye. This, the most difficult part of the examination, is extremely important, because it shows the most important part of the retina. As the corneal reflex is a very disturbing factor and the pupil contracts beeause the rays of light enter perpendicularly, the image is weakly illuminated and it requires great practise to see this part of the eye-ground clearly. The best plan is to ask the patient to look into the observer's left eye when it is desired to examine his left macula lutea. The surgeon then tries to get the image of the left optic nerve into the temporal portion of the convex lens, and turns the mirror very slightly toward the nose, whereupon the macula lutea becomes visible in the nasal portion of the lens and the troublesome corneal 
reflex disappears toward the temple. To examine the right macular region the patient is asked to look at the observer's right ear and the same steps are again repeated. Care must be taken not to allow any light to fall into the other eye, so as to avoid any contraction of the pupil, and if the pupil is too small in spite of this precaution it must be dilated.

It is of the greatest importance in using this method that the auxiliary lens be absolutely clean and free from scratches. Impurities in the glass always attract the beginner's eye and thereby make it difficult for him to see the eye-ground ; and, besides, there is danger that they may be mistaken for abnormalities in the eye-ground itself, so that things seem to be seen there which in reality do not exist. The most minute spots, such as the finger usually leaves on the glass, appear white in the eyeground and closely simulate white spots in the retina, for example, such as are found in albuminuria and diabetes.

After the eye-ground has been sufficiently studied by the indirect method, the surgeon proceeds to the fourth step : examination by the direct method. To do this most successfully, the lamp should be placed close to that side of the patient's head which corresponds to the eye to be examined. The beginner will do well to confine himself to the patient's right eye, which he can inspect with his own right eye, as most people find this the easier of the two. After placing the patient so that the lamp is on his right side, the surgeon simply throws the light into the eye. Then, still keeping the light in the eye, he gradually comes nearer and nearer, and finally as near as possible, until he begins to see in the red glare of the pupil details such as portions of blood-ressels in the eye-ground, taking good care, as explained above, to relax his accommodation. The observer should always imagine that he is looking through the patient's head into infinity. If the patient has been told to look straight ahead and to the left (into infinity), the surgeon will soon get a view of the optic nerve by tracing one of the retinal ressels back to 
it. The examination of the left eye is somewhat more difficult, because it must either be examined with the left eye to aroid collision between the observer's and the patient's nose, or the patient must turn his head away from the light. On the whole, it is best to learn from the beginning to use the right eve in examining the patient's right eye, and the left eye to examine the patient's left eye.

In looking for the erect image it is well to observe the following points: If the light is thrown on the patient's face by means of a concave mirror held at a distance of 5 em., ${ }^{1}$ and the observer looks through the opening in the mirror, he will see at the center of the brightly illuminated field, which has the same shape as the mirror, a dark, blurred spot which represents the image of the opening. This can be most easily studied by throwing the light on the patient's forehead. It will be noted that the pupil appears red as soon as this image of the opening is brought to coincide with it, hence if, without accommodating, we control the position of this dark spot as we approach the eye (when tho spot becomes more distinct), and by means of indirect vision keep this dark spot constantly on the patient's pupil, the latter will constantly appear red, and some portion of the eye-ground will become visible as soon as the proper point has been reached and the refracting media permit of the production of a distinct image. Hence it is not enough in the direet method simply to direet the light from the mirror into the patient's eye, but the center of the mirror, or, in other words, the opening must be made to coincide with the patient's pupil, because this is the only way in which the rays leaving the patient's eye can enter the observer's eye.

- If correcting lenses are necessary it is better to insert them without removing the mirror from the eye, that is, by simply turning the disk of lenses. By doing this the observer will learn to keep his accommodation relaxed.

It is a very good plan to begin one's studies in ophthal-

$$
{ }^{1} \text { With a plane mirror } 6 \text { to } 10 \mathrm{~cm} \text {. }
$$


moscopy on the rabbit, and I have, therefore, given an illustration of the rabbit's optic nerve and surroundings in this book (Fig. 6,a). The rabbit's pupil is naturally large, and the eye is usually at rest; besides, the eye is hypermetropic, and the emerging rays therefore diverge, making it easier to obtain a direct image.

Should we use the plane or the concave mirror in the direct method? I should answer this question by saying that both the plane and the concave mirrors have their advantages. But the examiner should make himself perfectly familiar with the color-gradations in the eye-ground, especially the optic nerve, with at least one of them. I cannot say that abnormal colors, such as pallor of the optic nerve or gray discoloration, can be seen better with the plane than with a good concave mirror, but if two mirrors are used, the question is complicated by the fact that it becomes necessary to train one's self to recognize a special set of abnormal colorings for each mirror. Hence if the student uses only one nirror, he will obtain a certain proficiency in diagnosis earlier. In any event, it is best always to use the same mirror for the direct method. I have no doubt, however, that the examination of the macula will be more exact if a concave mirror is used. A concave mirror brings out the macula itself and any alterations that may be present better than does the plane mirror, for the simple reason that in examining this portion of the eye-ground the pupil is contracted, and, owing to the darker pigmentation of the macular region, the image is dark enough even with the concave mirror, and altogether too dark and indistinct if a plane mirror is used.

\section{THE NORMAL EYE=GROUND.}

The appearances of the normal eye-ground as seen with the ophthalmoscope present, as I have stated in the beginning, so many variations that a thorough knowledge of the normal conditions is of the greatest importance before proceeding to a study of the pathologic alterations. 
I cannot too strongly advise the beginner to study normal eyes as often and as thoroughly as possible.

But an accurate knowledge of the anatomic structure of the various parts is equally necessary for a proper understanding of the normal conditions in the eye-ground. These are explained in Figs. 2, 3, and 14.

The first thing we notice on looking into the eyeground is the peculiar red coloration, which is very often misinterpreted and described as "abnormal redness." It is due to the fact that the choroid, as its name implies, possesses a great number of blood-vessels, especially in the anterior layer next to the retina-viz., the plexus of the choriocapillaris-which becomes more and more dense as we approach the posterior pole. The red color of the blood in these vessels is chiefly responsible for the red color of the eye-ground. It is partly due also to the color of the retinal vessels, although they play a very insignificant part. The purple color of the optic nerve has very little to do with it. We cannot see it as such any more than we can appreciate a delicate rose tint on a deep red color, not to mention the fact that the purple color of the optic nerve is very faint or disappears altogether under illumination.

But the color of the eye-ground varies considerably in intensity. In some individuals it appears a light yellowish-red, in others the color varies from a dark red to a brownish-red. This variation is due to the variable amount of pigment contained in the eves. Brunettes, as a rule, have darker eyes than blondes. The pigment may be variously distributed. The more intense the pigmentation of the retinal pigment-epithelium, the more uniform will be the dark red color of the eye-ground, because the red color of the choroid is more or less obscurred and the choroidal vessels cannot be seen. In other individuals the pigment may be chiefly in the choroid, especially between the ressels, the epithelium of the retina being sparingly supplied with pigment. Again, the choroidal vessels do not always stand out with 
equal distinetness behind the retinal vessels. In some eases we only see dark areas which, on closer examination, turn out to be pigmented "intervaseular spaces of the choroid" (Figs. 1, 6, $a, 9, a, 22$, etc.). In other cases the entire plexus of retinal vessels reveals itself at once (Fig. 5, $b$ ), owing to the presence of pigment between the vessels. If there is an entire absence of pigment in the retina and choroid, as in the albino, the vessels of the choroid appear red on a white ground (Fig. 10, $b$ ). The latter corresponds with the selera. In individuals who, without being absolute albinos, possess very little pigment, as blondes, for instance, the choroidal vessels are occasionally seen as red lines on a light red ground (Fig. 4).

In regard to the pigmentation of the background, it is to be remembered that the region about the posterior pole corresponding to the macula and the neighborhood of the optic nerve is usually darker than the peripheral portions (see Fig. 1 and many of the other pictures).

The dark brownish-red spot in the middle of the macula is due chiefly to the thinning of the retina at that point (see Fig. 14). As a result the pigment of the pigment epithelium and of the choroid is seen more distinetly through the attenuated nembrane. The choroidal pigmentation is deepest where the choroid joins the optic nerve; and at this point we sometimes see more or less distinctly a black ring, the so-called choroidal ring (Figs. 1 and 5, etc.). Sometimes there is a clear space between the optic nerve and the choroidal border, forming a more or less complete white ring, owing to the sclera appearing between the nerve-substance and the choroidal border. Hence this white ring is known as the scleral ring (Figs. 1 and 5, etc.). The substance of the optie nerve in the ophthalmoscopic image appears grayish-red and partly transparent, the nasal portion being somewhat darker than the temporal, owing to the greater aggregation of nerve-fibers emerging in the nasal portion. 'The center is frequently, but not always, the lightest. This depends 
on the presence or absence of a more or less developed excavation in the center (Fig. 2, a). The deeper this excavation, the more apparent the white lamina cribrosa in the center, and the brighter, therefore, the corresponding area. The optic nerve of the rabbit has a very distinct excavation (Fig. 6,a). It shows very plainly the manner in which the vessels distribute themselves over the retina without emerging from the funnel-shaped excavation. When the excavation is very deep, so that the lamina cribrosa is plainly seen at the bottom, it is called a physiologic excavation (see Figs. 5, $b, 54, b$ ). The lamina cribrosa is recognized as a collection of gray dots on the floor of the excavation. The border is formed by a delicate rim traversed by the vessels as they dip down into the excaration.

The optic nerve is not always circular in outline; it may be normally somewhat oval in either direction. Under normal conditions it does not project beyond the retina, or if it does, only to a very slight extent in the nasal third, hence it is very much better to use the term optic disk, rather than to describe it as a papilla.

The retina being transparent, except where it is covered by pigment-epithelium, is almost invisible in the ophthalmoscopic image. Occasionally a delicate striation of nervefibers is visible about the optic nerve, especially above and below it. In youthful individuals with darkly pigmented eye-grounds it is usually possible to see morlerately well-marked reflexes on the anterior surface of the retina about the fovea centralis and the optic nerve. They appear as grayish-white, irregular spots among the vessels, or as bright lines along the vessels, and very often, even in later years, as a ring about the fovea centralis (see Fig. 5, a), or a small ring, or small bright sickle at the center of the fovea.

-We recognize these bright spots as reflexes by their peculiar sheen and by the fact that their shape and position are affected by the movements of the mirror. They are most marked when the indirect method is used, especially if the pupil is not well dilated. 
The strongest of these reflexes is seen about the fovea centralis, and unless the observer looks directly on the macula he will only see a semilunar portion of it, usually the nasal half first. If the entire reflex is seen at once, it usually appears as an oval with its long diameter in the transverse direction and its vertical diameter corresponding approximately with that of the optic nerve, while the margin of the oval is sharply defined toward the center of the fovea and gradually disappears toward the periphery. Occasionally this reflex appears circular. The oval form cannot, as stated by Johnson, be due to the fact that the fovea centralis itself in most cases has a slightly oval shape, for, as I have frequently observed, the shape of the oval in any case of well-marked oval reflex is not affected by placing the lamp above the patient's head. Within the macular reflex the eye-ground is usually very dark, the coloring being deepest at the center where there are no reflexes, with the exception of a minute point at the center surrounded by a dark foveal spot (see Figs. 1 and $5, a)$.

The macula can be examined only by means of the direct method, which gives a magnified image. By this method it is seen that the central reflex point is formed by a small shining sickle, which moves about the center of the fovea as the observer moves his head, in such a way that the points of the sickle are always directed toward him. If the observer looks into the eye along the nasal border of the corneal reflex, the points of the sickle will be directed toward the temporal side, and if he looks along the temporal border of the corneal reflex, the points of the sickle will be directed toward the nasal side, etc. If the observer can succeed in looking directly into the eye along the foveal axis, that is to say, as much as possible through the corneal reflex, the sickle will change to a small ring about equal in size to the largest venous trunk of the optic disk. The bright semitransparent corneal reflex is very disturbing in this examination, because it lies immediately in front of the central portion of the macula. 
If the pigmentation is only moderate, we see in the region of the macula lutea, especially near its center, in the region of the somewhat darker foveal spot, a delicate stippling of the eye-ground, giving the appearance of a mosaic composed of minute light and dark spots. This stippling simply represents the irregular pigmentation of the pigment-epithelium of the retina. It may be more or less visible in the entire eye-ground if the pigmentation is not very marked.

Dimmer, ${ }^{1}$ who studied the reflexes on the anterior surface of the retina with great care, has pointed out that the smaller foveal reflex is due to the regular reflection of the light at the central, that is the deepest, portion of the retinal pit, and represents an inverted image of the sickle-shaped or circular portion of the mirror which lies nearest the opening. For the center of the retinal pit forms a small concave mirror (see Fig. 14, a-c) which reflects the light in such a way that it can emerge from the pupil under examination. But the rays that are reflected from the edge of the retinal pit (that is to say, peripherally from the foveal reflex) cannot leave the pupil, hence we usually see no reflexes in the remaining portion of the pit, which accordingly appears dim. Occasionally we see an additional, somewhat larger, luminous ring, concentric with the foveal reflex. Beyond the border of the pit the rays of light are again reflected in such a way that they can leave the pupil, hence the bright reflex of the macular ring which has been mentioned. The sharp central border of this oval, therefore, corresponds to the point where the retina begins to become thinner to form the pit; or in other words, to the sides of the pit. It follows that the macular reflex exactly corresponds with the outline of the central retinal pit, and can therefore be utilized to determine the size of the latter. Since the optic disk has an average diameter of $1.5 \mathrm{~mm}$., the vertical diameter of the fovea is approximately the same, while the horizontal is somewhat greater, disregarding individual variations

${ }^{1}$ Dimmer, Die Ophthalmoscopischen Lichtreflexe der Netzhaut, 1891. 
that occur here as well as in the diameter of the optic nerve. With the direct method and an ordinary concave mirror, the large foveal reflex (marginal reflex) appears very faintly or not at all, owing to the small amount of light illuminating the eye-ground in this method. It appears a little more distinctly if a strong concave mirror-one of $8 \mathrm{~cm}$. focal distance, for instanceis used.

The other reflexes of the retina, especially those that follow the course of the vessels (see Fig. 5, $a$ ), are formed very much in the same way as the macular reflex. According to Dimmer's investigations (loc. cit.) they are produced by concave cylindrical or spheroconcave surfaces found on the inner surface of the retina. These surfaces project at a certain distance inverted images of those portions of the ophthalmoscope from which the light reaches them.

In the region of the retina our attention is next arrested by the blood-vessels. The first point that we notice is that they all meet on the optic disk, as the retina receives its entire blood-supply from the arteria centralis retince, which, with the vena centralis retince, occupies the axis of the optic nerve at its entrance into the globe. Both the artery and the vein break up into their branches either on the optic disk or (sometimes) before they reach it. The arteries are recognized in the ophthalmoscopic image by the fact that they have a lighter color and a little brighter and broader central stripe than the veins. These differences in color can only be seen, however, in the coarser branches, just as the reflexes appear much more plainly in the thicker vessels and in the stronger magnification of the direct method. The bright stripe along the center of the vessel, according to Dimmer (loc. cit.), is due in the case of the arteries to the reflection of the light by the blood-corpuscles in the axial stream, and in the case of the veins to a similar reflection by the anterior surface of the bloodcolumn. Hence the middle stripe in the arteries is red- 
dish, while that in the veins, being a simple reflex, is white. $^{1}$

The course of the arteries is, on the whole, straighter than that of the veins. The central artery, like the central vein, subdivides into a variable number of branches in the last segment of the optic nerve, or in the excavation, or upon the optic disk. The two principal branches in both systems run at first up and down; soon they subdivide again, so that we can make out an inferior and a superior temporal artery and vein. In some cases we can also distinguish a nasal artery and vein carrying the blood to and away from the nasal portion. The important region of the macula is supplied in part by vessels coming directly from the optic nerve and in part by branches of the inferior and superior temporal artery and vein which arch around the macula. Hence we usually see in the macula and its immediate surroundings only delicate vessels radiating more or less toward the center, although it is impossible to trace them to the center of the forea, even in the direct method. They usually disappear from sight near the inner border of the macular reflex. That they do reach the center of the fovea, however, is shown by the well-known entoptic arterial figure elicited by moving a small opening to and fro in front of the eye, or by moving a candle from side to side in the dark.

In the peripheral portions of the retina the vessels are few in number, of minute ealiber, and mostly radiate toward the periphery. The arrangement of the ressels is atypical in the following cases:

(1) When there is a so-called cilioretinal vessel. In that case a delicate artery appears at the temporal border of the optic disk. It arises in the choroid, passes into the region of the opticus, enters the retina, and there

${ }^{1}$ In the pictures of this volume it has been, for technical reasous, impossible to reproduce these vascular reflexes accurately, especially in regard to width and color, not to meution the fact that with the slight magnification of the indirect method the reflexes are at best very indistinct. 
pursues a general direction toward the macula. This condition is not very rare and, when present, is usually found in both eyes.

(2) When there is an opticociliary vessel. This is much rarer. It is a branch of the central vein or central artery and does not run as far as the retina, disappearing at the border of the optic disk. In other words, it does not reach the vascular plexus of the choroid.

(3) Instead of the venous blood from the choroid being carried off by the vortex-veins in the equatorial region (Fig. 10,b), we often see, in highly myopic eyes, posterior vortex-veins which have a similar, but less extensive ramification and carry off the choroidal blood at the edge of the optic disk (Fig. 45, a). Why these posterior vortex-veins should be found so often in highly myopic eyes I cannot as yet understand.

\section{Pulsation Phenomena.}

The pulse-phenomena seen in the eye-ground call for a careful description. When we look at the richly branching ressels of the eye-ground for the first time, we cannot help asking ourselves why they lie so rigid and immovable and give no sign of pulsation, unless it be an occasional beat at one or more of the extremities of the veins on the optic disk. The reason why we usually cannot see any distinct pulsation in the arteries of the retina, or only a very slight pulsation, is, in the first place, the fact that vessels of such minute size show little or no pulsation because the pulse-wave has become too weak by the time it reaches a small artery. It must never be forgotten that we see the eye-ground greatly magnified with the ophthalmoscope, and that if we examine the globe with the naked eye, the retinal arteries even where they are widest, that is, on the papilla, appear only as delicate red lines.

Another reason commonly given for the absence of pulsation in the retinal arteries is the intraocular pressure which counteracts the pulse-wave. 
It is, however, possible, with great care, to see true pulsating movements in the retinal arteries of normal individuals, but only where the course of the artery is curved.

After a series of careful examinations I have reached the conclusion that if one of the larger retinal arteries in the region of the papilla is distinctly curved we can always see a movement of pulsation, manifesting itself as a bulging of the arch with the systole or as a slight to-and-fro movement at the center in a direction perpendicular to the chord of the are. If there are two curves in succession, forming a letter $\mathrm{S}$, the phenomenon is even more distinct. It is also brought out more clearly by vigorous heart-action. This pulsatory locomotion is more easily and more frequently seen than is the pulsatory variation in caliber, or, in other words, the alternate expansion and contraction of the blood-vessels. The latter phenomenon may be seen in heart cases. Another form of locomotion-a pulsatory backward and forward movement of bifurcations in the larger arterial branches-is also seen in cases of heart-disease.

On the other hand, there is a kind of artificial pulsatory movement, best described as an intermittent influx, which can be produced in any normal eye. If, while carefully looking at the papilla, a gradually increasing pressure is exerted with the finger [on the eye under examination] we can see the to-and-fro pulsation of the blood-column in the ends of the arteries on the papilla. This phenomenon can be observed even by the indirect method, but, like all pulse-phenomena in the eye-ground, appears more distinctly when the direct method is used. By the latter we also see how the intermitting influx is produced. As soon as the pressure of the finger reaches a certain degree, the ends of the arteries on the papilla are emptied, and at the same instant we notice that a little blood is forced into these vessels only at the height of the pulse-ivave, after which the ressels collapse until the next cardiac systole, so that the ends of the arteries 
are empty for that space of time. The ends of the veins, of course, do not show any pulsation. On the contrary, if there is a physiologic venous pulse it usually subsides and the ends of the veins are less distended, the pulse reappearing only after pressure on the eye is removed, when the ends of the veins swell out and even the finer vessels on the papilla appear more distended and stand out more distinctly than they did before pressure was made on the eye.

This intermittent influx of the arterial blood into the vascular system of the retina is observed also when the pressure in the interior of the globe is raised by disease, instead of artificially by means of the finger. It occurs in glaucoma whenever the pressure rises rapidly (acute glaucoma); it is less noticeable in simple glaucoma where the rise of pressure is gradual. The intermittent influx is exactly the same as when the pressure is raised artificially. The pulse-wave overcomes the pressure on the walls of the vessels only at the moment of its greatest height.

A similar phenomenon is said to occur when there is pressure on the arteria centralis retinæ behind the bulb, as, for instance, in the case of a neoplasm.

Analogous to the arterial end-pulse-wave is a venous end-pulse, better described as an intermittent efflux. This phenomenon is somewhat more difficult to explain. As has been said, the pulsating movement is often noticed in normal eyes, and then has no significance. Three different explanations have been offered. Donders ${ }^{1}$ assumed that the pressure in the arteries of the interior of the eye was somewhat heightened during the cardiac systole, so that the ends of the veins on the papilla were compressed, because in them the lateral blood-pressure is least marked. As soon as the cardiac systole is over the venous blood flows off. Helfreich ${ }^{2}$ gives the following explanation: According to investigations by Bergmann and Cramer it

1 Donders, Arch.f. Ophth, vol. i.

2 Helfreich, Arch.f. Ophth., vol. xxriii. 
may be assumed that, owing to the rhythmical increase in the amount of blood sent to the arteries of the brain, the blood is crowded out of the cerebral veins by compression and this gives rise to an increase in the rate of flow of the venous blood from the cranium synchronous with the pulse. Hence we have marked pressure-variations in the venous sinuses of the brain. The pressure-variations in the cavernous sinus necessarily influence the movements of the blood in the veins of the orbit and of the interior of the eye. Whenever the pressure in the cavernous sinus diminishes, the cerebral blood is, as it were, aspirated, and there results a diminution in the caliber or a partial collapse in the orbital veins, providing the walls of the veins are not so firmly adherent to the surrounding tissues as to preclude the possibility of their collapsing. Certain conditions are, however, necessary for the pulse to show itself, otherwise the phenomenon would be constant.

Both of these theories in regard to the origin of the venous end-pulse may be utilized. v. Schulten has shown by manometric experiments that there is a slight increase of the pressure in the interior of the eye synchronous with the cardiac systole. Anyone can satisfy himself with the ophthalmoscope that a slight increase in pressure, as by the finger, is immediately followed by a diminution in the amount of blood in the ends of the veins on the papilla. The lightest touch of the finger rhythmically applied suffices to imitate the venous pulse. On the other hand, we may believe with Helfreich that the cardiac diastole is accompanied by an increase in the rate of flow of the blood from the orbital veins to the cavernous sinus; but whether this increase is sufficient to cause the veins on the papilla to collapse, as contended by Helfreich, may be disputed. While it may be admitted that the blood-pressure in these veins is very low at the end of the cardiac diastole, and that the increase in the intraocular pressure synchronous with the cardiac systole (Donders) momentarily contracts the veins perceptibly 
or even causes them to collapse, it is probable that the damming back of the venous blood from the brain (Helfreich) antagonizes this contraction of the veins and produces engorgement of their extremities, which, as everyone admits, manifests itself shortly after the cardiac systole. In this way the actual conditions on which both theories are based are utilized. Whether my explanation is correct or not must be decided by future investigators.

S. Türk ${ }^{1}$ offers a third explanation for the physiologic venous pulse. According to him it is due to a continuation of the arterial pulse-wave through the capillaries into the veins, a so-called progressive venous pulse, the pulsatory dilatation being produced by the cardiac systole, as in the arteries. According to him this abnormal prolongation of the pulse-wave is made possible by the comparatively high extravascular pressure to which the ressels in the eye are subjected.

A true arterial pulsation is seen in aortic insufficiency. In this condition the difference in the blood-pressure during systole and diastole in the arterial system is abnormally great; that is to say, owing to the cardiac hypertrophy, the pulse-wave is abnormally high and followed by an abnormal recession of the wave, because the blood flows back into the heart through the insufficient valve, unless aortic stenosis is also present. Accordingly, the smaller arteries and even the capillaries pulsate in this disease. In well-marked cases, we can see not only a violent pulsating of the retinal arteries and veins, but even a pulsatory blushing and paling of the papilla. The larger arterial branches in certain enditions show a true pulsation, both variation in caliber and locomotion, at a considerable distance from the papilla. As a rule, the veins in such cases show pulsation (variations of caliber), which may even be more marked than in the arteries. But if there is only slight insufficiency and but little hypertrophy of the left ventricle, or if the cardiac action 1 Türk, Arch.f. Ophth., vol. xlviii. p. 3, 1899. 
is merely weak, as when the patient is at rest, the pulsation in the retinal vessels may be absent or may appear only when the patient causes his heart to work faster. The intensity of the phenomenon is also diminished when there is co-existent aortic stenosis. In other forms of heart disease the retinal vessels rarely or never exhibit pulsation. An arterial pulse is occasionally observed in mitral insufficiency. In mitral stenosis I saw it only once in fifteen cases. In mitral stenosis complicated with aortic insufficiency, $I$ saw it in one case and not in another. Even in stenosis of the mitral valve and in mitral insufficiency with stenosis, I did not see any pulsation.

It appears, therefore, that the pulse-phenomena in the eye-ground are of very little value in the diagnosis of cardiac disease. Besides, the detection of pulse-phenomena in the eye-ground is one of the most difficult things in ophthalmoscopy. 'To study them carefully it is well to examine the patient on a chair instead of in bed, and to let him lean his arm on the table. The surgeon should, if possible, also be seated, and support the arm that holds the mirror on the table. If these precautions are neglected, the pulsating movements of the surgeon's arm or trunk will deceive him when he looks into the eye and make him think that the ressel he is examining is pulsating. The same error may be produced by pulsating movements in the trunk of the patient. Autosuggestion also plays an important part. If the observer is very anxious to see a pulsating vessel, he will seem to see things pulsate which in reality do not show a trace of pulsation.

It goes without saying that these things can be seen - only in the direct method. 




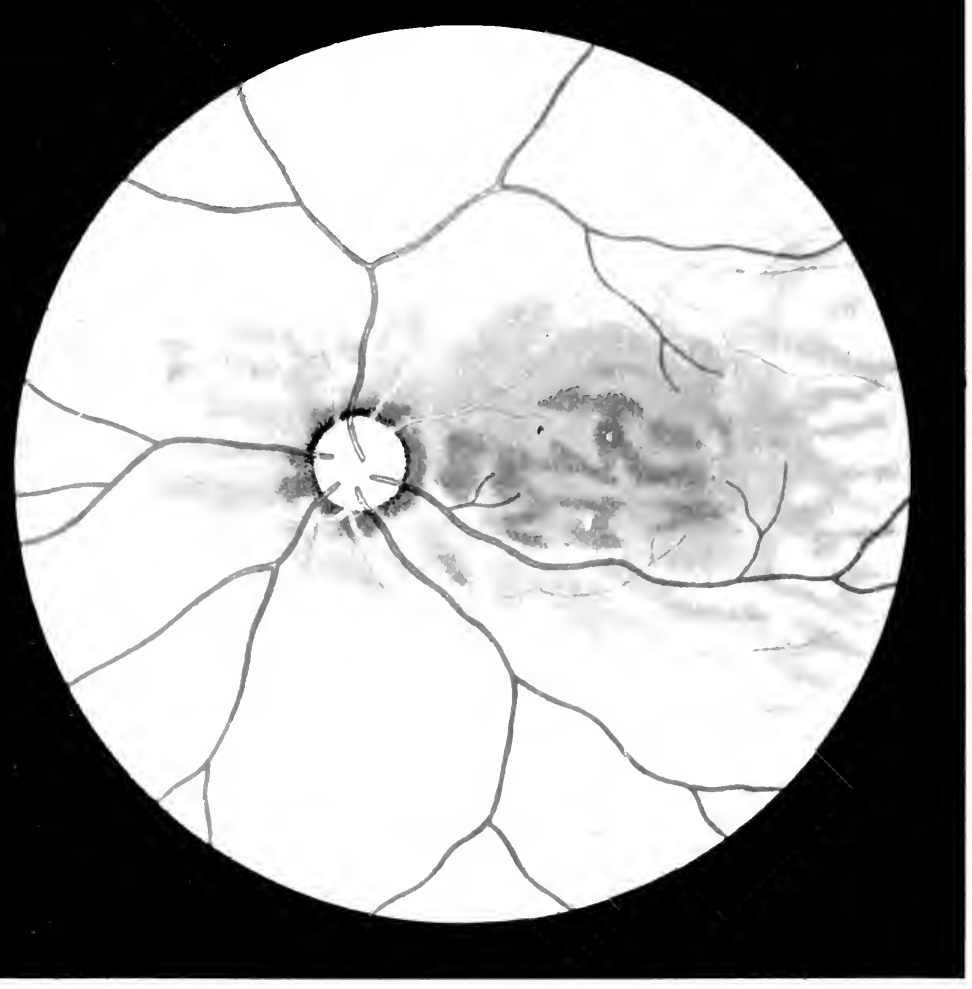

Fig. 1. 
Fig. 1. Normal Eye-ground.-A moderately pigmented eye-ground, the pigmentation being strongest about the optic nerve and behind the macula and its surroundings. At the periphery is seen the mottled appearance due to the increased amount of pigmentation in the intervascular spaces of the choroid. In front of the latter, in the center, are the retinal vessels with their lines of reflection. The arteries are lighter in color than the veins. The optic nerve possesses a moderately deep excavation with a correspondingly light color, a scleral ring, and a somewhat blurred choroidal ring.

Inexperienced observers often fall into the error of mistaking the dark intervascular spaces in the normal eyeground, or the bright striations between the dark islands for pathologic conditions, and are thus led to make the diagnosis of disseminated choroiditis. It is, therefore, well to study the appearances of the normal eye-ground, especially the appearance at the periphery. 
FIg. 2, a. Longitudinal Section of a Normal Papilla stained with Weigert's stain, showing how the nerve-fibers of the optic nerve lose their medullary sheath (black color) as they pass through the lamina cribrosa and thus diminish the caliber of the optic nerve. The blogd in the vessels of the optic nerve, the retina, and choroid, as well as In the neighborhood of the optic nerve, wherever it is present, is also stained black. In the region of the papilla the central vessels (V.c.) are in part imbedded and appear like round cells.

When the nerve-fibers retain their medullary sheath until they reach the retina-a condition which is constant in the rabbit and occurs occasionally in man, although only to a slight degree-a white radiation about the papilla is produced which is represented in Fig. $6, a, b, c$. The medullated nerve-fibers then appear glistening white in the ophthalmoscopic image. They are not transparent, and therefore obscure the retinal vessels in places.

In this picture there is a well-marked excavation where the nervefibers diverge after passing through the lamina cribrosa, differing in this respect from the following picture, where the divergence is very slight (cf. Fig. 2, b).

In the ophthalmoscopic image this appears as a pallor of the center of the papilla, or the so-called physiologic excavation-paler because the white, glistening connective tissue of the lamina cribrosa is seen more plainly through the diverging nerve-fibers.

The size of the excavation varies greatly within physiologic limits. When it is large, it is often difficult to decide whether we have to deal with a physiologic or pathologic, that is to say, glaucomatous cup.

$V$. c., central vessels; Pig., pigment-epithelium of the retina.

Magnified 14 times.

Fig. 2, b. Longitudinal section through a Normal Papilla showing Almost No Excavation.-The head of the nerve barely projects above the level of the retina, so that there is only a very slight papilla. Behind the depression, which is very shallow, the arteria et vena centralis retinæ can be traced for a short distance. Stained with hematoxylin and eosin. Nuclei and granules of the retina violet; connective tissue reddish.

$V . c$., central vessels; $J$., subdural space of the optic nerve between the dural $(D$.$) , or rather arachnoidal, and the pial (P$.) sheaths, traversed by trabeculæ of connective tissue from the arachnoidal sheath (cf. Fig. 21).

Magnified 14 times. ${ }^{I}$

I In the figures, which accurately reproduce the microscopical sections, intervals appear here and there between the retina and the choroid and between the choroid and the sclera. It is impossible to avoid this in preparing the specimens, but we must, of course, imagine the various layers in contact with each other. The same applies to many of the following figures. 


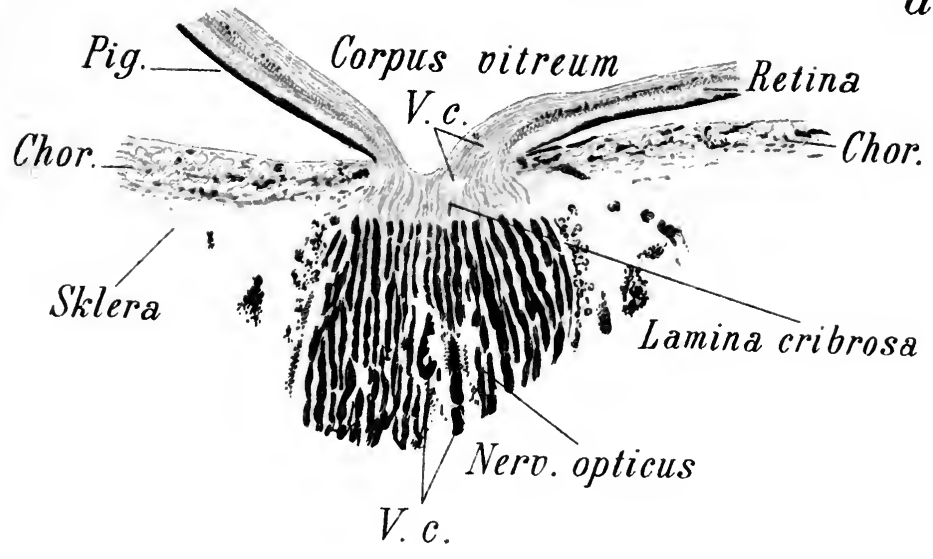

$b$

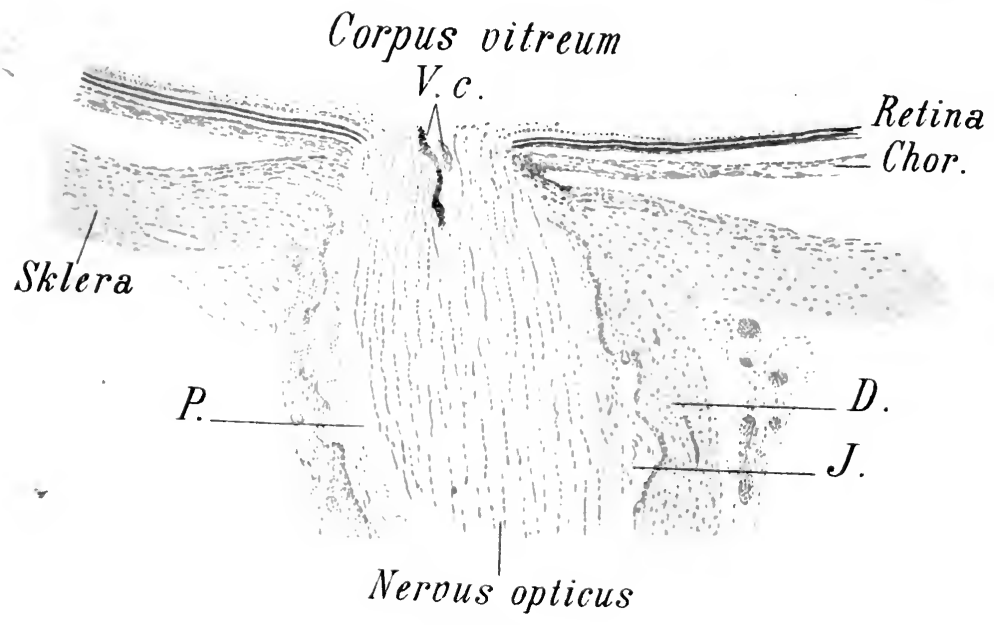

Fig. 2. 

Fig. 3. Section through the Retina, Choroid, and Contiguous Sclera in a Normal, Moderately Pigmented Eye at a distance of several millimeters from the macula lutea.The tissues were fixed by immersing the freshly enucleated globe in a warm saturated bichloride solution, hardened with alcohol, and stained with hematoxylin and eosin.

The preparation with the power used for this illustration does not show the individual fibers of the nerve-fiber layer. In the inner reticular layer Müller's supporting fibers are barely seen as fine red lines. In the internuclear layer the outer reticular layer is easily distinguished from the outer fiber layer. In the layer of rods and cones the outer segments of these structures are recognized by the fact that they are somewhat bent (probably an artefact), while the inner segments are placed vertically on the external limiting membrane. The nuclei of the pigment-epithelium of the retina are plainly seen. The blood in the choriocapillaris and in the other vessels of the choroid, as well as in those in the anterior layer of the retina, is stained bright red by the eosin.

In the choroid the pigment-cells are found chiefly in the intervals between the larger vessels (corresponding to the pigmented intervascular spaces of the ophthalmoscopic image; cf. Fig. 5, $b$, for instance). Only that portion of the sclera which is contiguous with the choroid is seen in the pictures of Fig. 2.

1 , Nerve-fiber layer; 2 , ganglion-cell layer; 3 , inner reticular layer; 4, inner nuclear layer; 5 , internuclear (outer reticular) layer; 6 , outer nuclear layer; 7 , layer of rods and cones; 8 , retinal pigment-epithelium; $V$., retinal vessels. - Magnified 214 times. 
FIg. 4. Normal Eye-ground.-The eye, being that of a blonde, possesses less pigment than that shown in Fig. 1. Note especially that the pigment-epithelium of the retina is much more transparent, so that the vessels of the choroid are very distinct. As the choroid also possesses a small amount of pigment, the intervascular spaces are lighter instead of darker than the vessels. The fovea in this case is red, and the reflex of the center of the fovea is not seen on account of the pale color of the eye-ground. In the optic nerve the excavation and the choroidal and scleral rings are very marked.

To understand the appearances of a normal eye-ground a knowledge of the normal anatomy of these parts is indispensable. The most important anatomic details are illustrated in Figs. 2, $a$ and $b, 3$, and 14, $a, b, c$. 


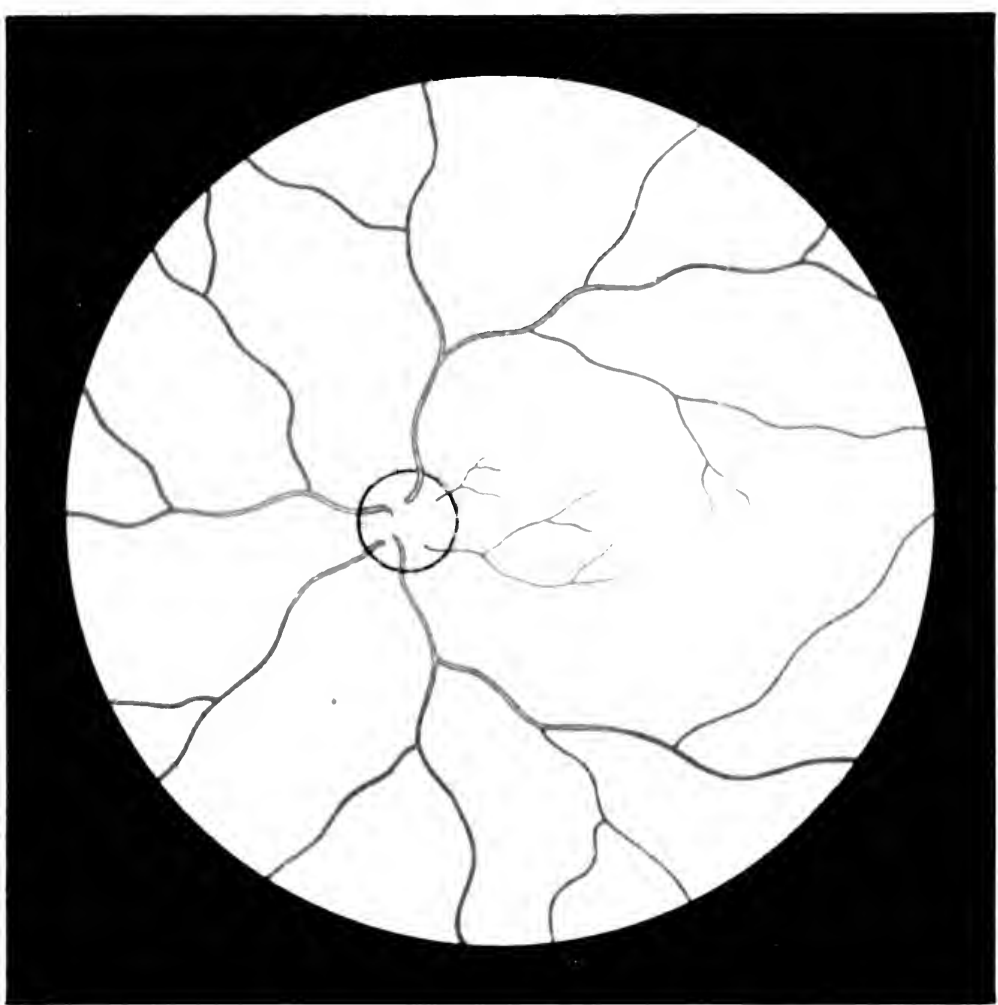

Fig. 4. 




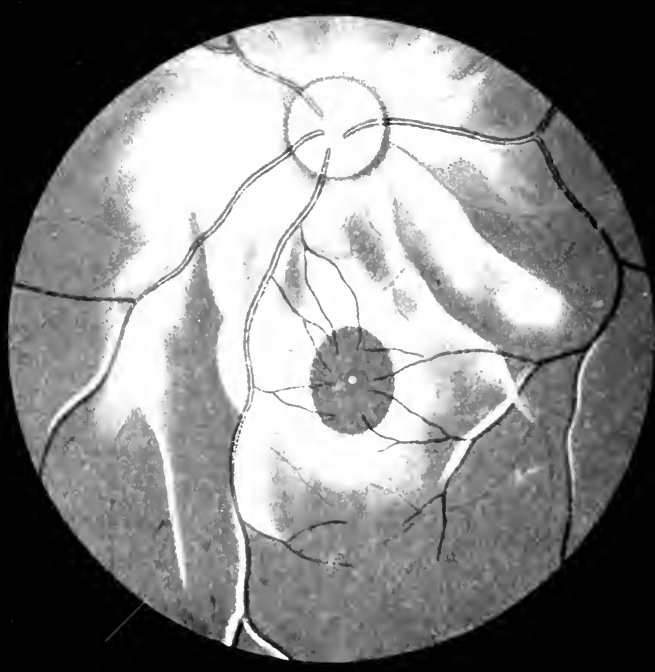

3

c.

er

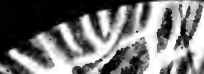

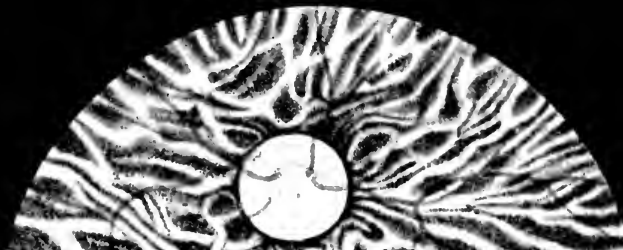

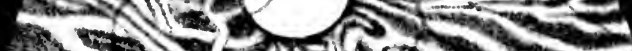

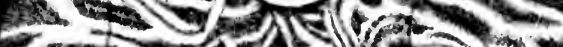

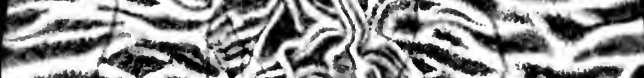

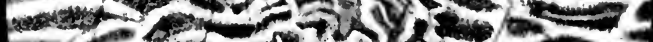

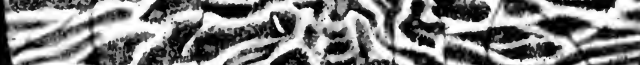

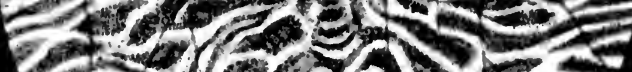

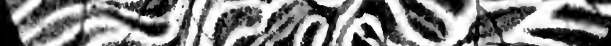

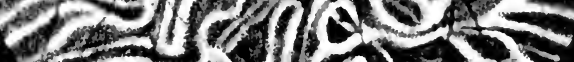

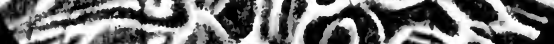

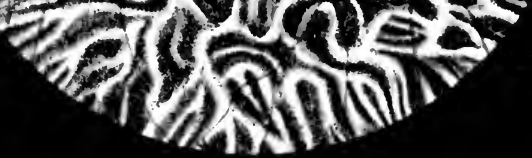




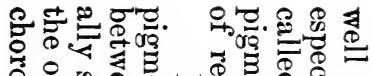

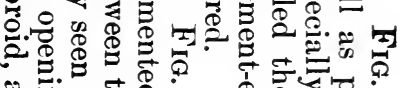

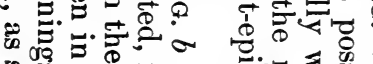

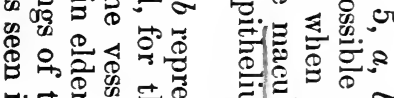

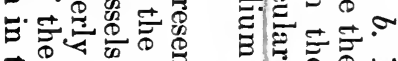
它. 它要

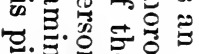

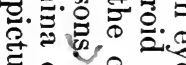
ड 3.

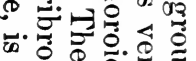

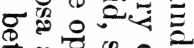

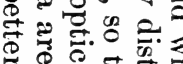

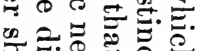
5.

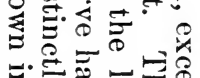
$\bar{\theta} \cdot \frac{0}{4}$

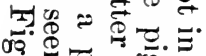

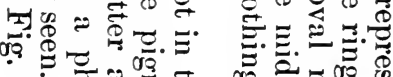

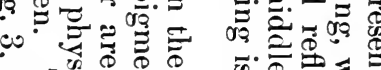

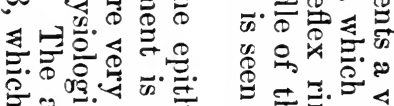

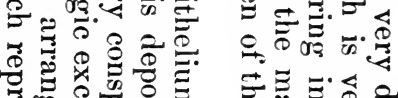
定骂 象 要

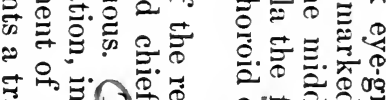

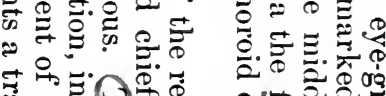

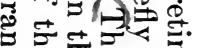

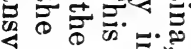

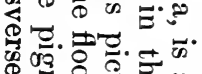
क 总造㶽

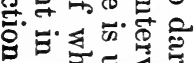

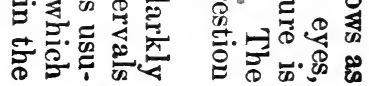




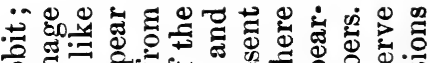

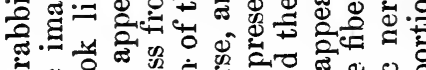
0 영

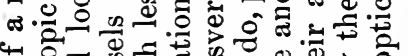
4t

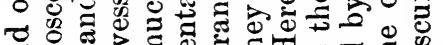

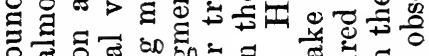

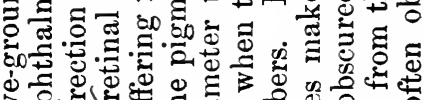

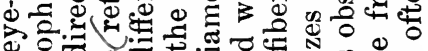

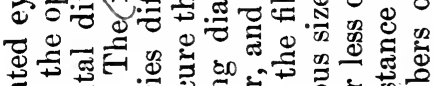

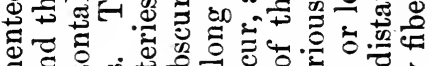

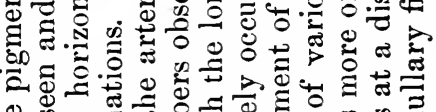
o

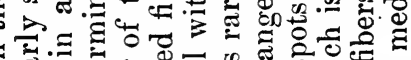

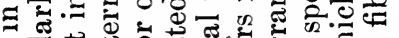
ᄋ

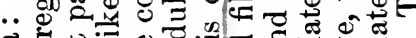

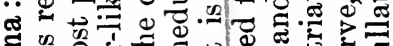

.2.

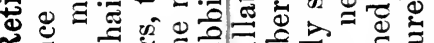

o

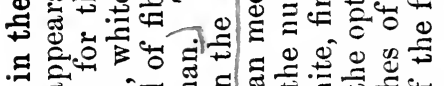

叫

蛅.

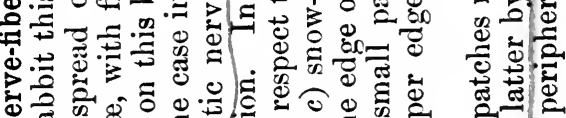

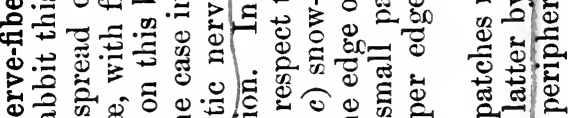
का के 0

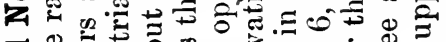

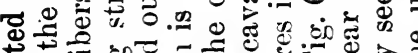

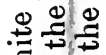

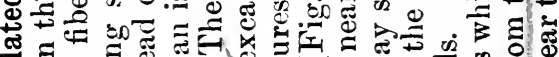

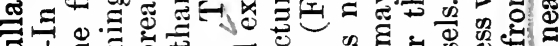

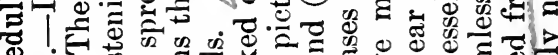

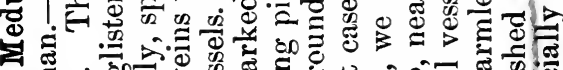

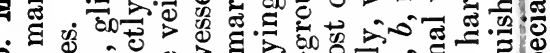
0 . c。 至 要言

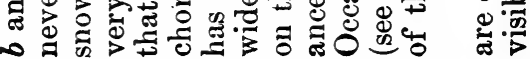




\section{CONGENITAL DEFECTS.}

(Figs. 7-11.)

Fig. 7. Congenital Circumscribed Defect of the Choroid (Coloboma Choroideæ) in the lower part of the figure (erect image reduced to the size of the inverted image).

The defect exposes the sclera and has a roundish outline, its upper border being at a distance of three opticnerve diameters from the papilla. Along this border a small amount of choroidal tissue is still present, but farther down the membrane is represented only by a few remnants in the form of pigment-spots and a few vessels. Some of the retinal vessels are seen on the surface of the coloboma. The rest of the eye-ground is normal.

These defects of the choroid in the lower segment of the eye have been attributed to persistence and failure to unite of the fetal cleft. This explanation, though simple, is not quite adequate. The fetal cleft lies in the region of the optic vesicle, which later becomes the retina, but as a matter of fact the retina is not absent in the region of the coloboma, since both in this and in the following figure retinal vessels are seen traversing the coloboma. The defect is therefore not in the optic vesicle and its cleft, but in the region of the mesoderm or of its derivative-the chornid. These inferior colobomas and other congenital defects (cf. the following figures) are probably due in many cases to intra-uterine disease, and it is probable that the fetal cleft plays but a secondary rôle in the first group, consisting of inferior coloboma of the iris and choroid. 
Fig. 8, $a$. Congenital Circumscribed Defect of the Choroid and Malformation of the Optic Nerve (Coloboma Choroideæ et Nervi Optici) (erect image reduced to the size of the inverted image).

The choroidal defect in this case surrounds the optic nerve, which is muchlincreased in size. The manner in which the vessels leave the optic disk is altogether abnormal. The optic nerve and the portion of the sclera joining it below are excavated (ectatic), the larger cavity containing three smaller diverticula, two oval and one round (staphylomata). A few retinal and choroidal vessels are seen in the region of the coloboma.

Fig. $8, b$. Congenital Defect of the Pigment-epithelium of the Retina in the Region of the Macula Lutea.-The two whitish patches correspond to defects of the choroidal tissue which expose the sclera. They are traversed by a few choroidal vessels. At the temporal border of the optic disk there is another defect of the choroid having the form of a triangle with the apex broken off. In the orange-colored areas, where the pigment-epithelium is absent, irregular lines of pigmentation are seen. The rest of the eye-ground is normal. The other eye shows similar changes.

The patient is a young woman suffering from hereditary syphilis. 


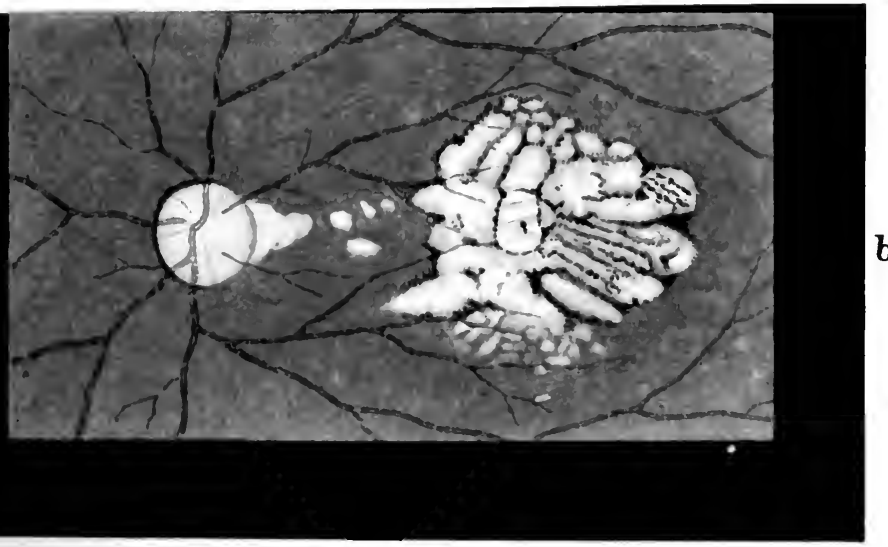

Fig. 8. 

0

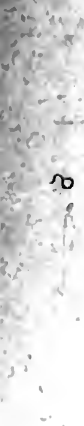

a 


$$
8
$$


용

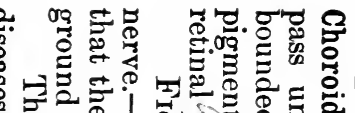
क्ष

จ

ช

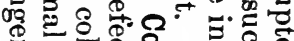

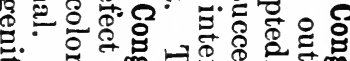

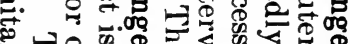

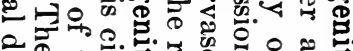

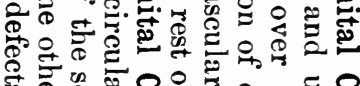

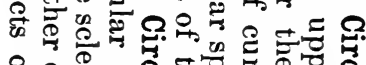
요 苯多

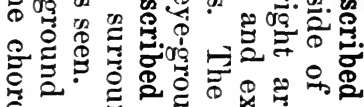
的

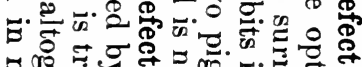
క ๙

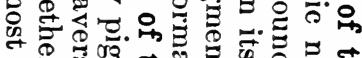
ใ广

\& 눙 -

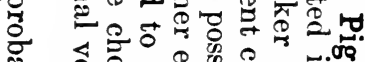

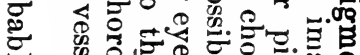

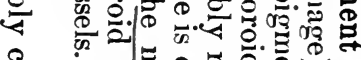

ڤ

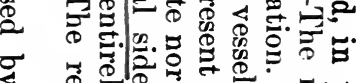

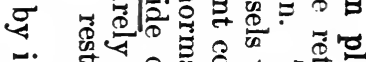

5. $\quad$ 눈

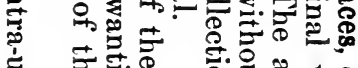

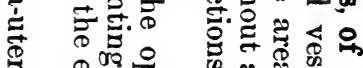

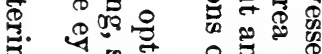

苛 की 


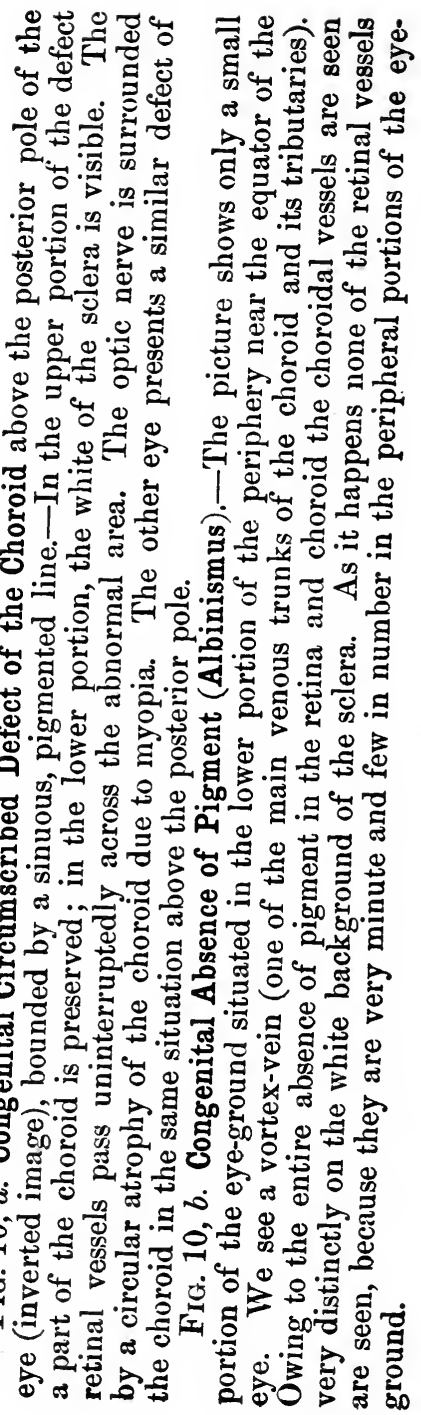


$\circ$

宇

$\sigma$ 



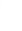

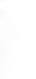

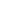

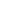
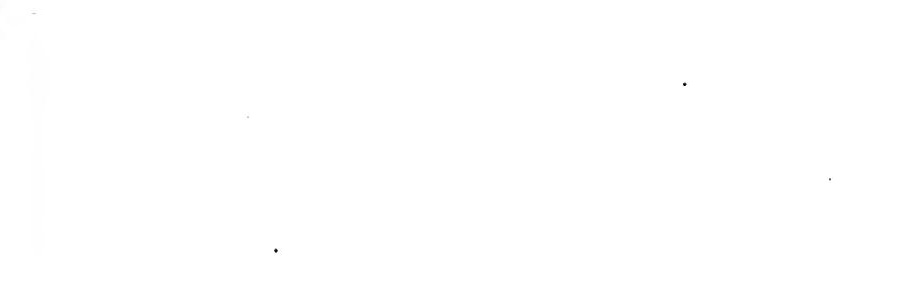


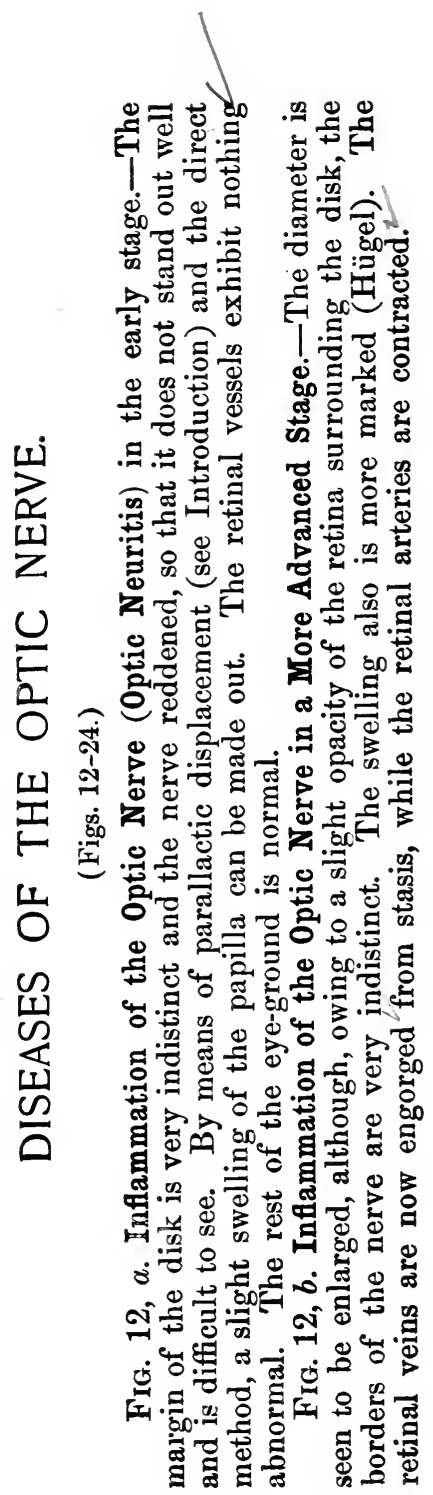




$$
8
$$






$$
0
$$


<smiles>CCCCC</smiles> 


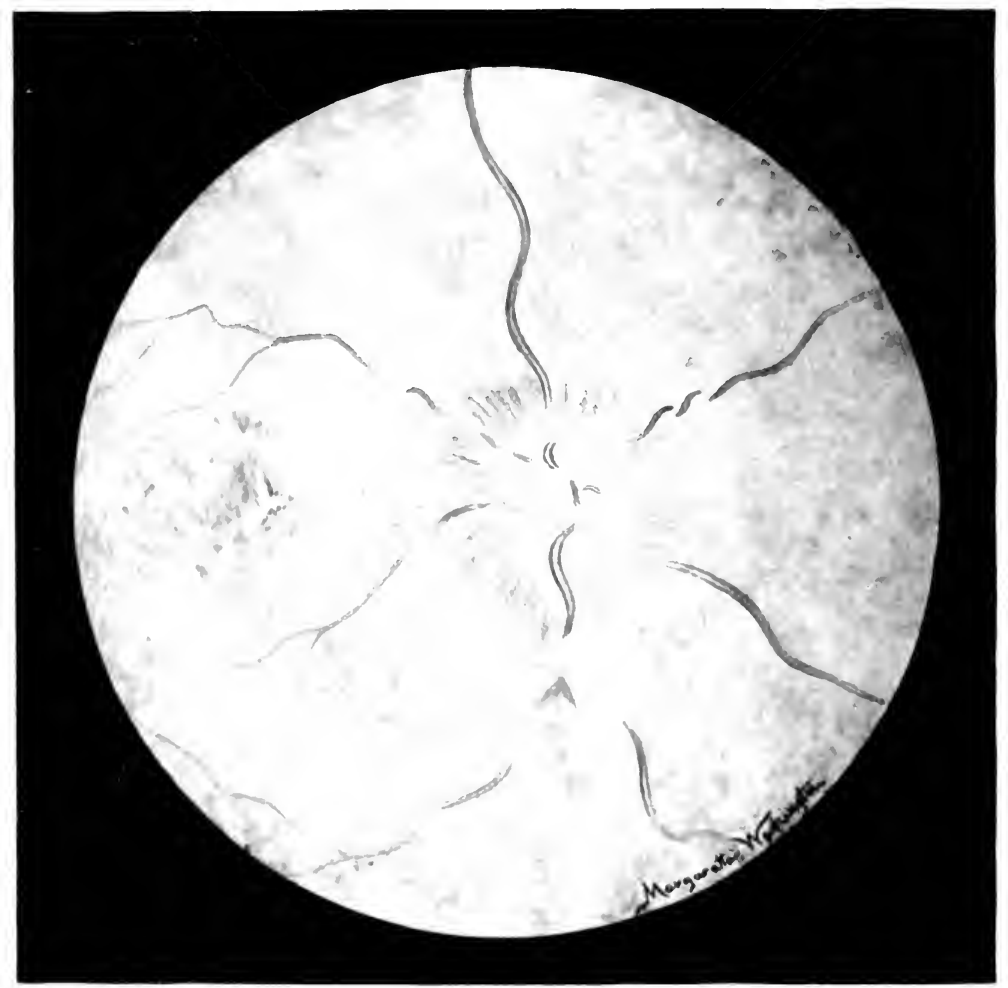

Firi. 18, 1.-Choked disc and macular changes, from a patient with tumor of the cerebellum (de fichweinitz). 


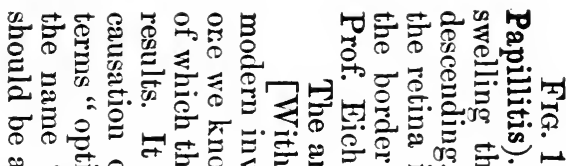

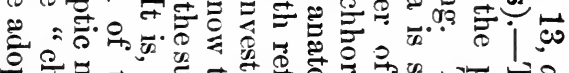

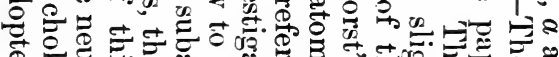

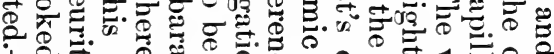

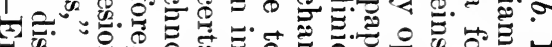

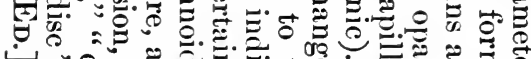
:

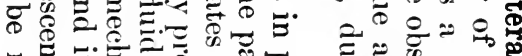

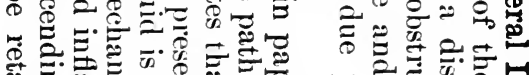

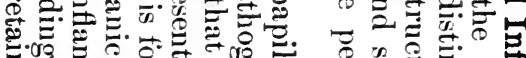

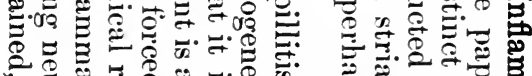
Q

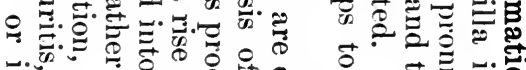

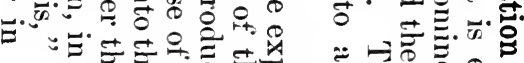

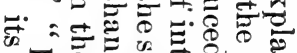

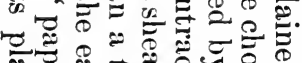

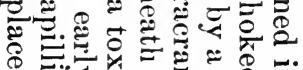

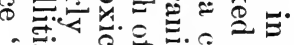

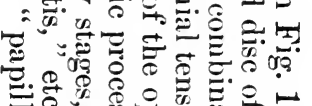

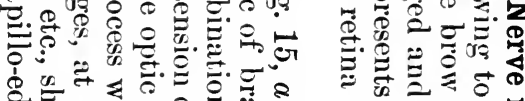
क

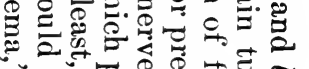

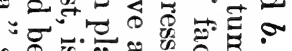

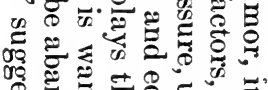

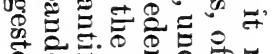

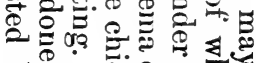

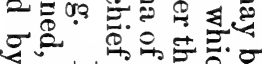

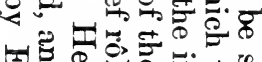

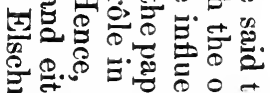

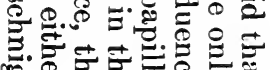

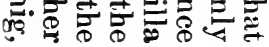

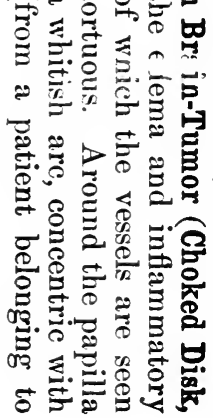


Fig. 14, a. Horizontal Section of a Normal Macula Iutea, almost exactly through the fovea centralis $(F . c$.$) .-The freshly$ enucleated globe was immersed in a warm saturated bichlorid solution and hardened in alcohol. The sections were stained with hematoxylin and eosin. The walls of the excavation are probably not so steep in the living subject as they appear in this preparation. On the floor of the excavation the pigmentary layer is reduced to the slender cones with their fibers and nuclei. In the internuclear layer the fibers, owing to the manipulation of the specimen, diverge somewhat (better seen in Fig. 14, c), which makes the walls of the excavation appear steeper than normal.

Magnified 14 times.

Fig. 14, $b$, shows the outlines of the same preparation, seen under the same power as the following picture. Magnified 30 times.

FIG. 14, c. Another section from the same specimen through the excavation and its surroundings, taking in the adjoining choroid and sclera, under a higher power than Fig. 14, $a$. (On the floor of the excavation there is a slight prominence which is an artefact.) Next to the pigment-epithelium we see the choriocapillaris. On the posterior surface of the sclera are seen several transverse and oblique sections of posterior ciliary vessels $(V$.$) . Stain the same as in Fig. 14, a$.

The picture incidentally shows the relative thickness of the three membranes-retina, choroid, and sclera.

Magnified 30 times.

In both sections we see at the margin of the fovea centralis the internal limiting membrane (margo limitans internus), which in the specimen has become somewhat separated from its foundation. 


\section{a \\ Corpus oitr. \\ F.c. \\ Retina

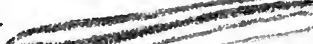

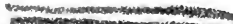

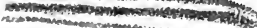

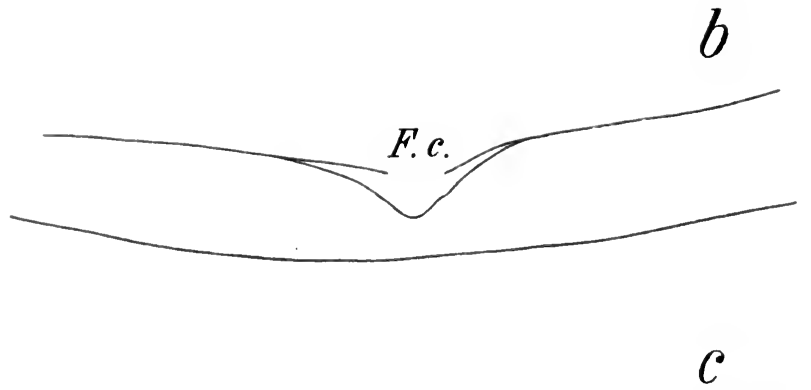

\section{Corpus oitr.}

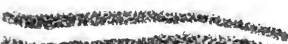

\section{F.c.}

势

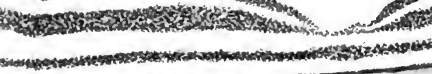
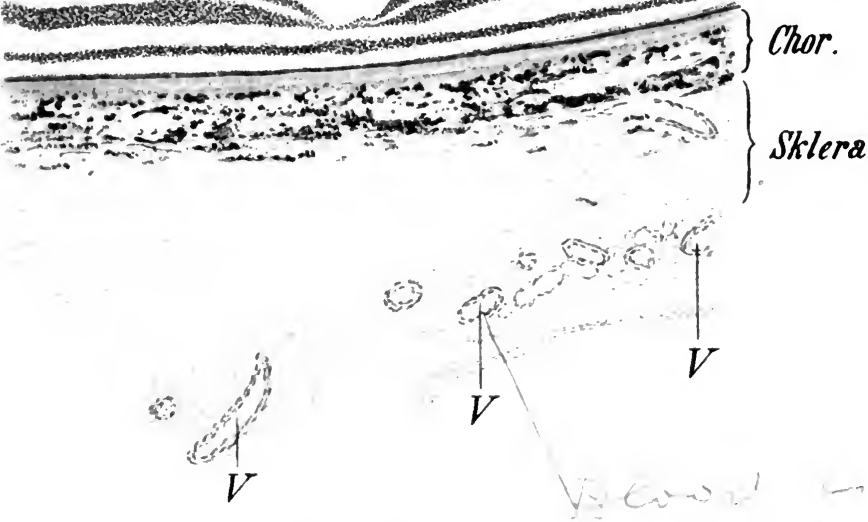

Fig, 14. 



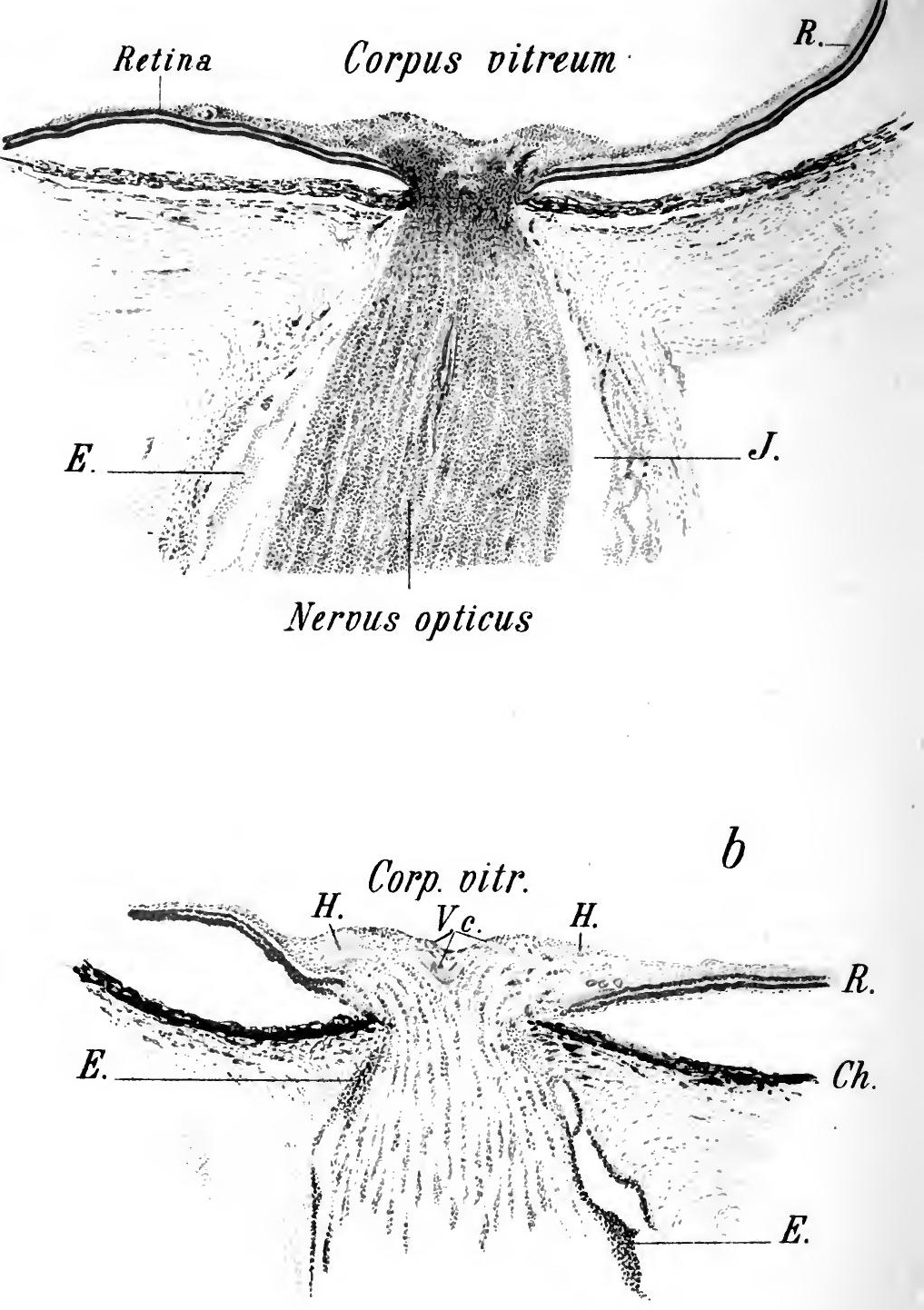

Fig. 15. 
Frg. 15, a. Longitudinal Section through the Papilla in Neuritis (Papillitis, Choked Disc), due to brain-tumor (sarcoma of the temporal lobe).- Stained with hematoxylin. The papilla in this specimen is moderately swollen. The swelling may be much more marked in these cases-even more marked than in the following picture. The separation of the retina from the choroid is an artefact. (See Ed. note opposite Fig. 13.)

There is a heavy inflammatory infiltration (proliferation of nuclei) between the lamina cribrosa and the anterior surface of the papilla. In the subdural space $(J$.) occasional areas of inflammatory exudate $(E$.) are also seen. The trunk of the optic nerve, in the center of which a third segment of the central vessel is visible, is also the seat of moderately well-marked inflammatory proliferation.

$E$., inflammatory exudate in the subdural space of the optic nerve; $J$., subdural space; $R$., retina.

Magnified 14 times.

In this case the papilla and surroundings first presented the picture seen in Fig. 12, $a$, and later that seen in Fig. $13, b$.

Fig. 15, $b$. Longitudinal Section through the Papilla in Neuritis and Papillitis from brain-tumor with purulent meningitis, terminating fatally in spite of trephining.- Stained with hematoxylin and eosin. The swelling of the papilla is greater than in the foregoing case and would be more pronounced were it not for the separation of the retina from the choroid (artefact).

The lateral extension of the swelling beyond the border of the papilla, which in the ophthalmoscope produces the socalled enlargement of the papilla (see Figs. 13, 17, and 18), is clearly seen. We also see without difficulty a marked engorgement of the vessels, especially of the reins of the papilla $(V . c$.$) and hemorrhages (H$.$) into the tissues of the$ papilla. There is also some inflammatory exudate in the subdural space.

$V . c$., central vessels; $E$., exudate in the subdural space; $C h$., choroid ; $R$., retina ; $H$., hemorrhages.

Magnified 14 times.

The ophthalmoscopic image in this case resembles that shown in Figs. 17 and 18. 
Fig. 16. Inflammation of the 0ptic Nerve and the Adjacent Portion of the Retina in Syphilis (so-called Specific Neuroretinitis).-There is a marked blurring of the optic nerve and its surroundings, due in part to the diffuse central opacity of the vitreous. The peripheral portions of the eyeground in this case are not diseased, but in many cases they are the seat of foci of disseminated choroiditis in various stages. The picture before us is characteristic, or at least strongly suggestive, of syphilis.

The opacity may disappear under appropriate treatment, but usually leaves a more or less pronounced atrophic discoloration of the nerve. Minute foci of choroiditis may develop in the periphery in the course of the disease. 


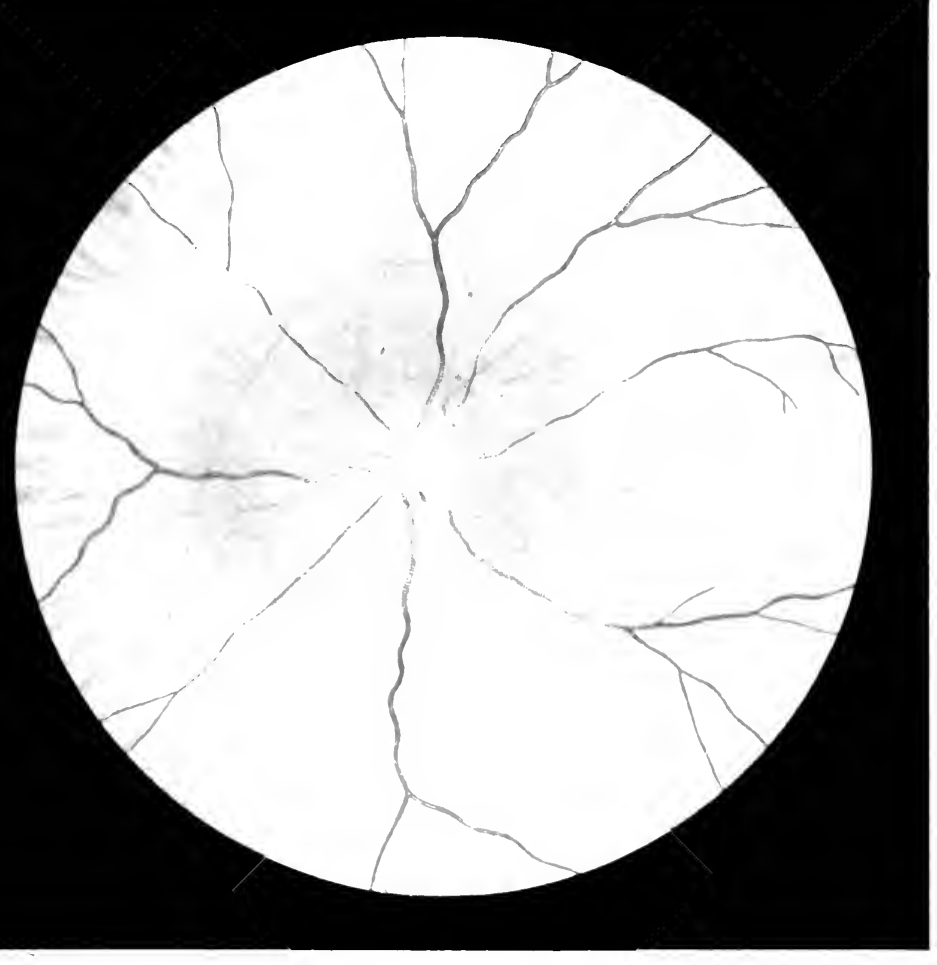

Fig. 16. 




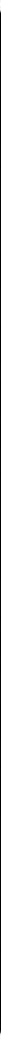

Fig. 17. 
Fig. 17. Intense Inflammation of the 0ptic Nerve (Papillitis) after meningitis caused by a blow on the head.-The inflammation in both eyes has led to marked infiltration of the tissues of the nerve, manifesting itself in grayish-white patches and striæ on the papilla and surrounding portions of the retina, and in the hemorrhages at the lower outer border of the discolored areas. The diameter of the nerve is enlarged and the nerve itself moderately swollen and prominent. Owing to the presence of inflammatory product in the tissues of the nerve, the venous flow from the retina is impeded and the veins are therefore distended and tortuous (patient belonging to Prof. Eichhorst's clinic).

The microscopic appearance corresponding to this picture would be about the same as that shown in Fig. 13, $b$. 


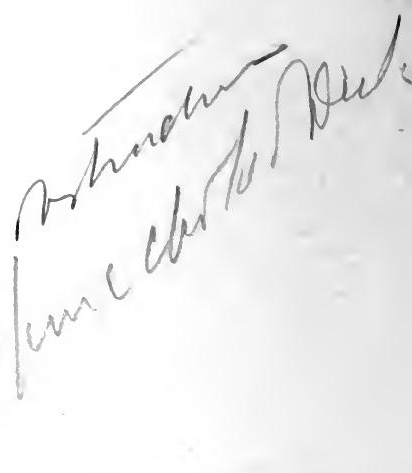

Fig. 18. Marked Inflammation and Congestion of the Optic Nerve in Orbital Tumor.-Exophthalmos (protrusion) was present. The intraocular extremity of the optic nerve shows marked inflammatory and edematous swelling in the picture. The inflammatory infiltration appears as a whitish striation. The veins of the retina are very much congested and numerous hemorrhages into the retina have occurred in consequence. The retinal arteries are moderately distended. 



$$
8
$$




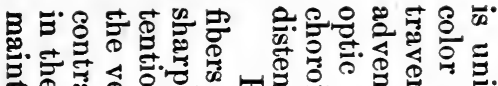

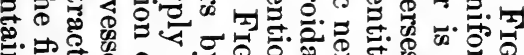

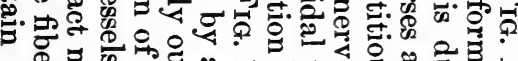

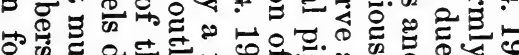

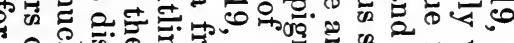

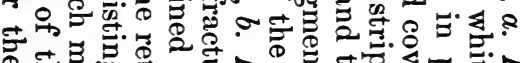
(

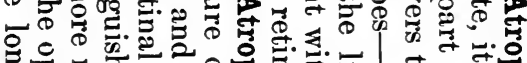

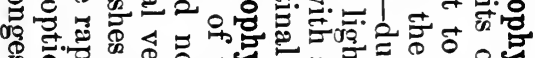
娄

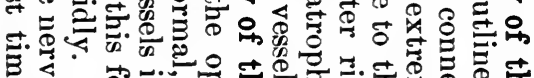
จ

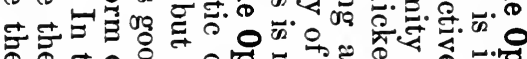

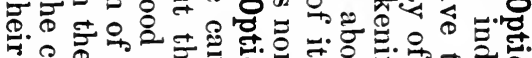

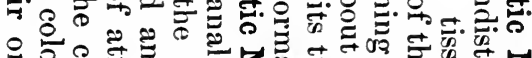

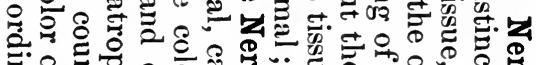

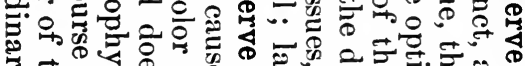

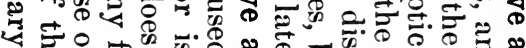

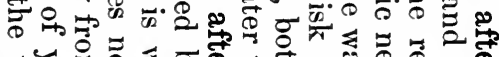

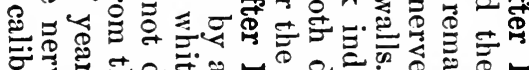

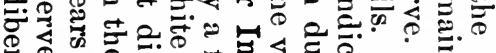
๑ के के

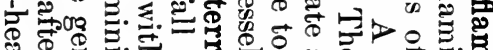

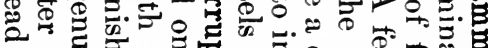
ข.5.

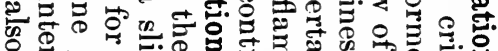

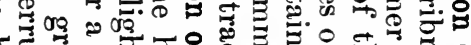

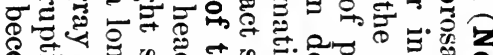
ঠ

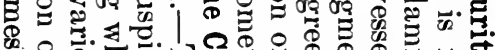

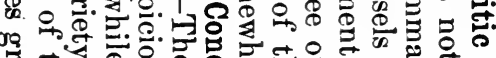

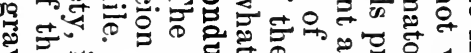
క.

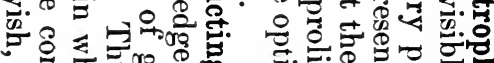

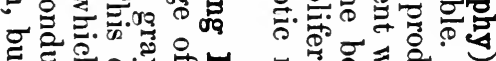

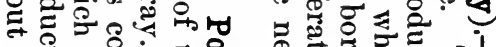

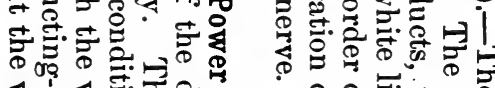
ه

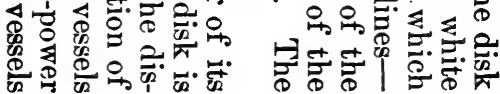




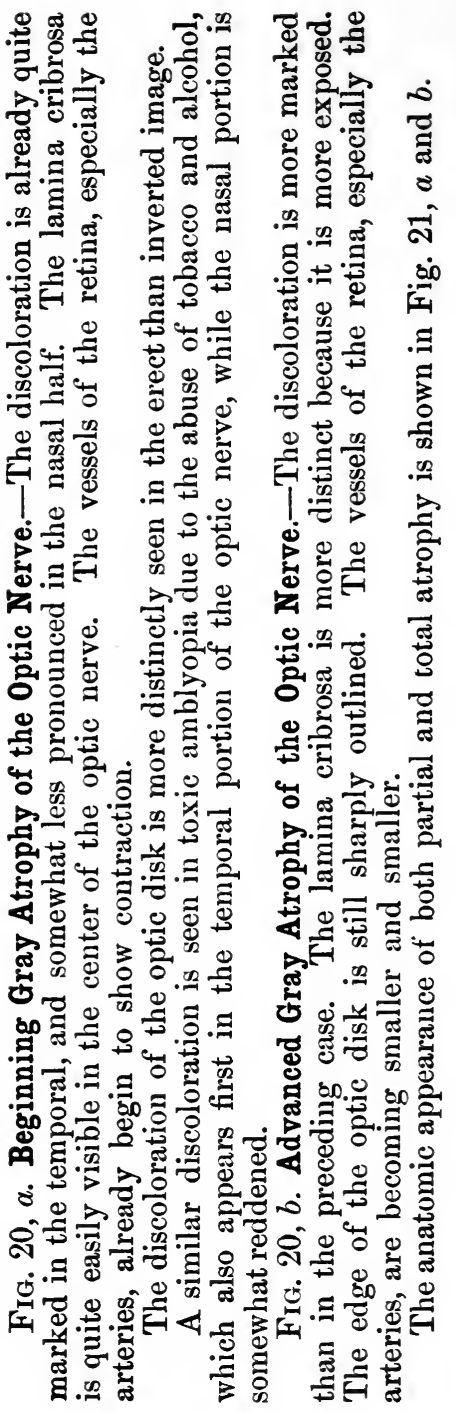




$$
8
$$




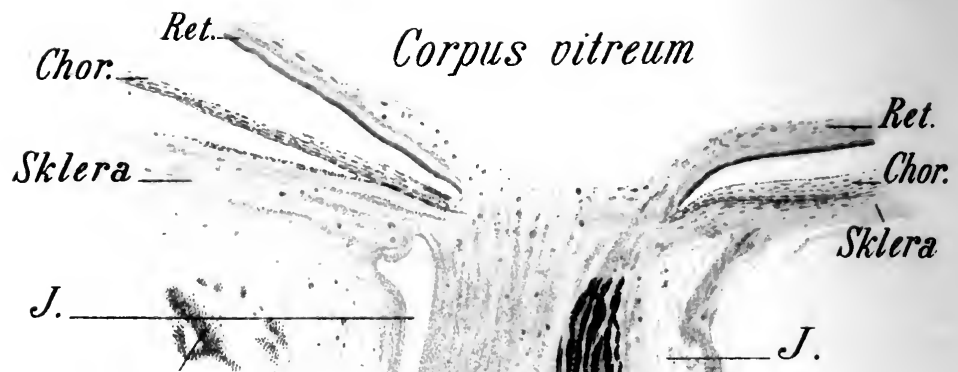

V.cil.

Arteria central.

A.

Vena centralis

Nerous opticus
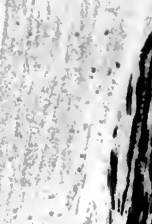

$J$

$D$.
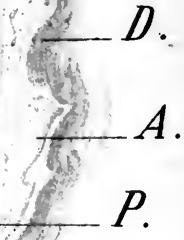

$b$

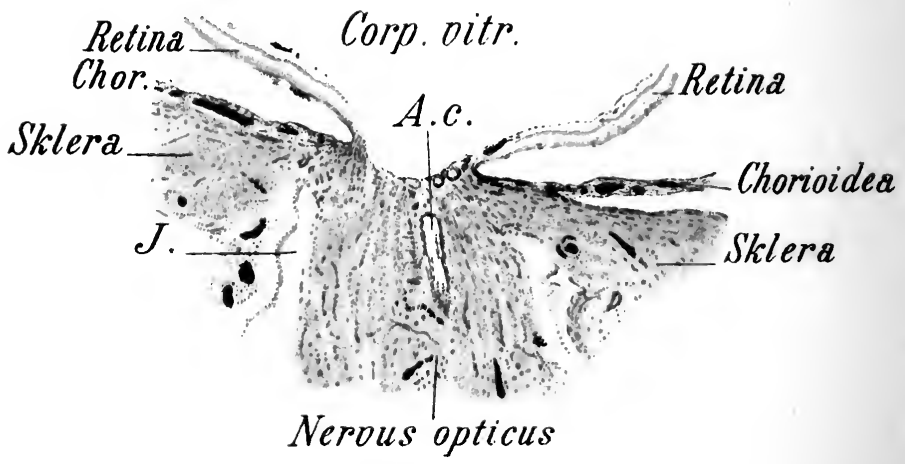

Fig. 21. 
Fig. 21, a. Longitudinal Section through the Entrance of the 0ptic Nerve in Partial Atrophy (of the 0ptic Nerve).Stained by Weigert's method, which colors the medullary sheaths a bluish-black. In the atrophic left half of the nerve-trunk the medullary sheaths are altogether absent. Owing to the partial atrophy and consequent diminution in the volume of the nerve, the subdural space is somewhat broader than normal and the arachnoidal sheath is plainly visible. The papilla is already distinctly flattened from atrophy.

$D$., dural sheath; $A$., arachnoidal sheath ; $P$., pial sheath ; $V$. cil., ciliary vessel.

Magnified 14 times.

FIG. 21, $b$. Longitudinal Section through the Disk in Total Atrophy of the Optic Nerve, stained after Weigert.All the medullary sheaths are wanting. The trunk of the nerve is thinner even than in the preceding case. The papilla shows a distinct atrophic excavation, on the floor of which the lamina cribrosa is laid bare. A portion of the arteria centralis $(A . c$. ) is seen in this section.

This is the microscopic appearance that corresponds to the ophthalmoscopic picture shown in Fig. 20, $b$. The patient from which the specimen was taken presented at the time of his first examination a beginning gray atrophy like the picture of Fig. 20, $a$, and later advanced gray discoloration of the papilla, as shown in Fig. 23. Later he developed progressive paralysis and died in the insane asylum. The posterior half of the globe was kindly given me by Prof. Forel.

Magnified 14 times. 
FIG. 22. Atrophy of the Optic Nerve due to Increased Intraocular Tension (Glaucoma).-The entire end of the nerve shows marked excavation and dark discoloration. The lamina cribrosa is pushed back and plainly visible owing to the disappearance of the nerve-fibers. The choroid near the optic nerve is atrophied and forms a pale areola, also known as the glaucomatous halo. The retinal vessels are sharply bent at the edge of the excavation and dip down to the floor, where some of them become visible on the lamina cribrosa. At first the veins are congested and dilated, but now the vessels of the retina are also beginning to atrophy. Parallactic dislocation and measurement in the erect image (see Introduction) reveals a distinct movement of the edge of the excavation in front of the floor, and the excavation is found to be about $2 \mathrm{~mm}$. deep ( $6 \mathrm{D}$ difference of refraction between the edge and the floor).

The glaucomatous changes in the end of the nerve are illustrated in Fig. 24, $c$ and $d$. 

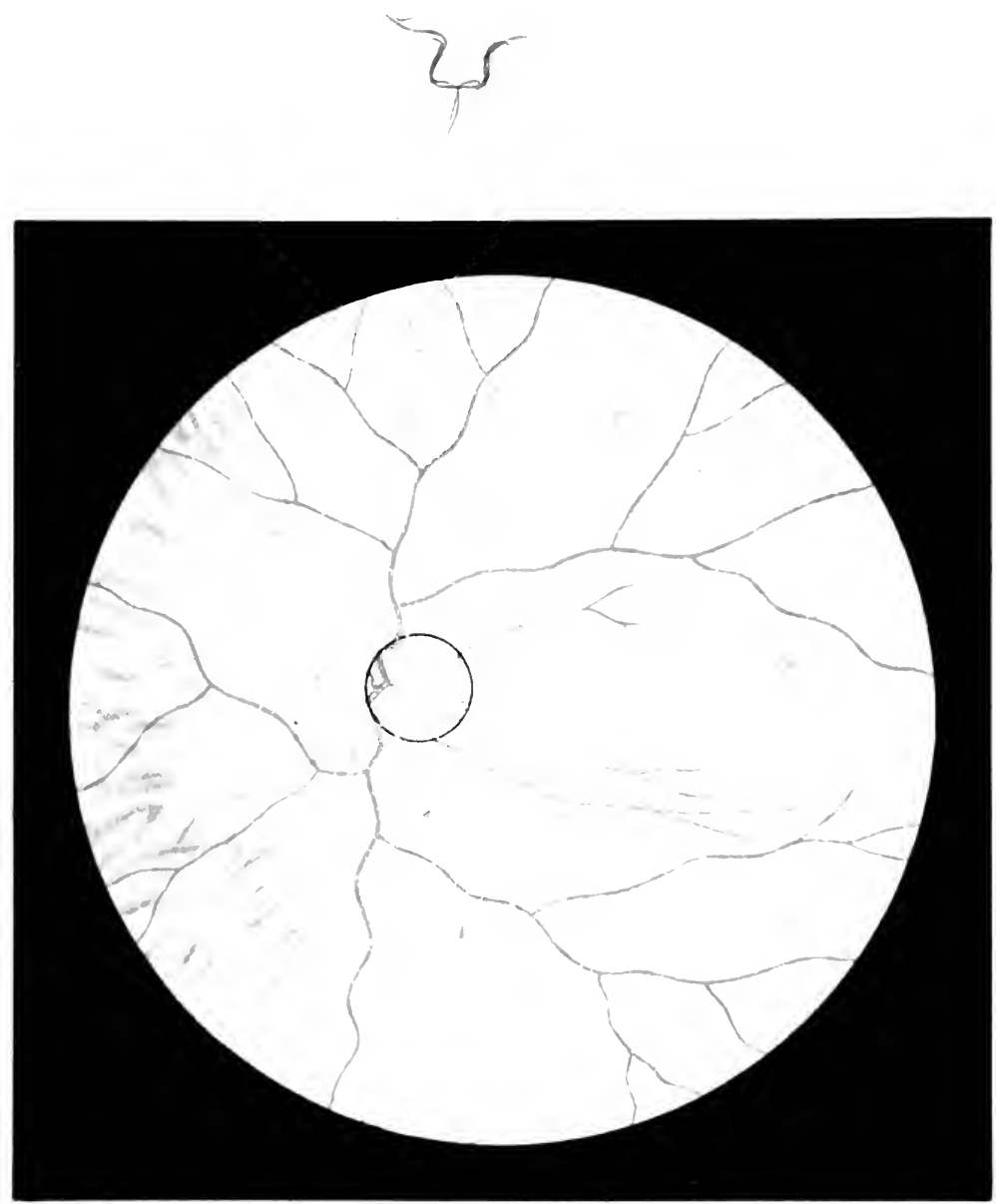

Fig. 22. 




$$
8
$$


言若.

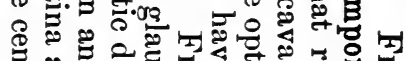

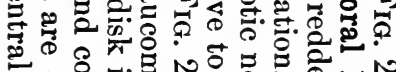

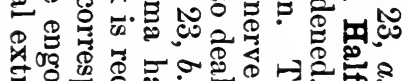

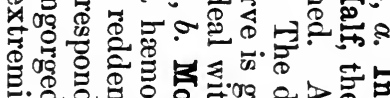

두은웅

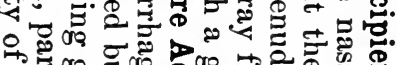

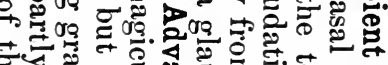

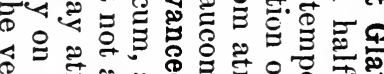

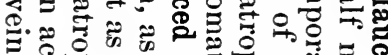

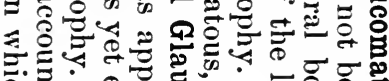

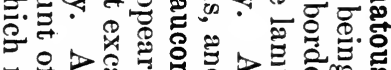

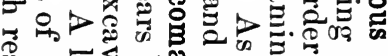

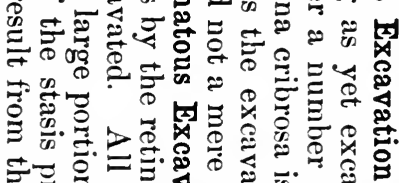

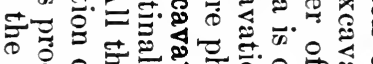

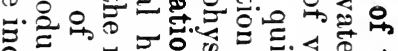

常

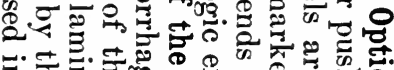

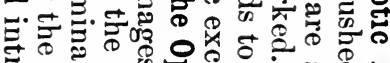

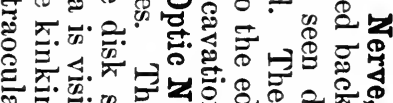

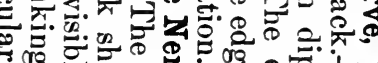

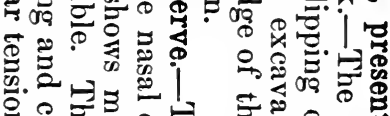

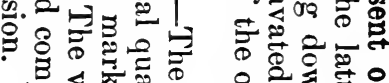

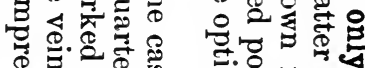

定.

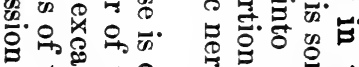

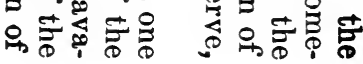


Fig. 24, a. Meridional Section through the Region of the Angle of the Anterior Chamber in a Normal Eye.-The ciliary body became loosened from the sclera during the preparation of the specimen, but the position of the iris is approximately correct, so that the angle between its ciliary attachment and the cornea is well seen.

C. v. c., circulus venosus ciliaris, or Schlemm's canal, or, in the new nomenclature of the Anatomical Society, sinus venosus scleræ; L.p., ligamentum pectinatum.

Magnified 14 times.

Frg. 24, b. Section through the Same Region, showing the Obliteration of the Angle of the Anterior Chamber which often occurs in Glaucoma.-As the aqueous humor leaves the bulb through the angle of the anterior chamber, obliteration of that angle obstructs its outflow and thus explains the increased pressure in glaucoma. The fluid is retained within the bulb and this gives rise to the further symptoms of glaucoma, especially the excavation of the head of the nerve by pressure, which is shown in the two following pictures.

Magnified 14 times.

FIg. 24, c. Longitudinal Section through the Head of the Optic Nerve in Advanced Glaucoma.-The excavation of the end of the nerve due to the increased intraocular pressure is well marked. The papilla is replaced by a concavity with steep sides, the floor of which is formed by the lamina cribrosa and the nerve-fibers which have not as yet become atrophied by the pressure and kinking at the edge of the papilla.

FIG. 24, $d$. Longitudinal Section through the Head of the Optic Nerve in a still more Advanced Stage of Glaucoma, showing the diminution in size of the trunk of the optic nerve produced by atrophy of the nerve-fibers and the increase in the width of the subdural space [of the optic nerve].- In this section the kettle-shaped excavation on the end of the nerve that occurs so often in glaucoma is quite pronounced. As the nerve becomes smaller between the lamina cribrosa and its anterior extremity, as shown in Fig. 2, $a$, the glaucomatous excavation in which the lamina cribrosa is pushed backward by the abnormal pressure upon it is very apt to take on this kettle shape, that is to say, to become narrower in front than behind. To a limited degree this may be seen in the preceding figure.

Fig. 24, $c$ and $d$, magnified 14 times. 

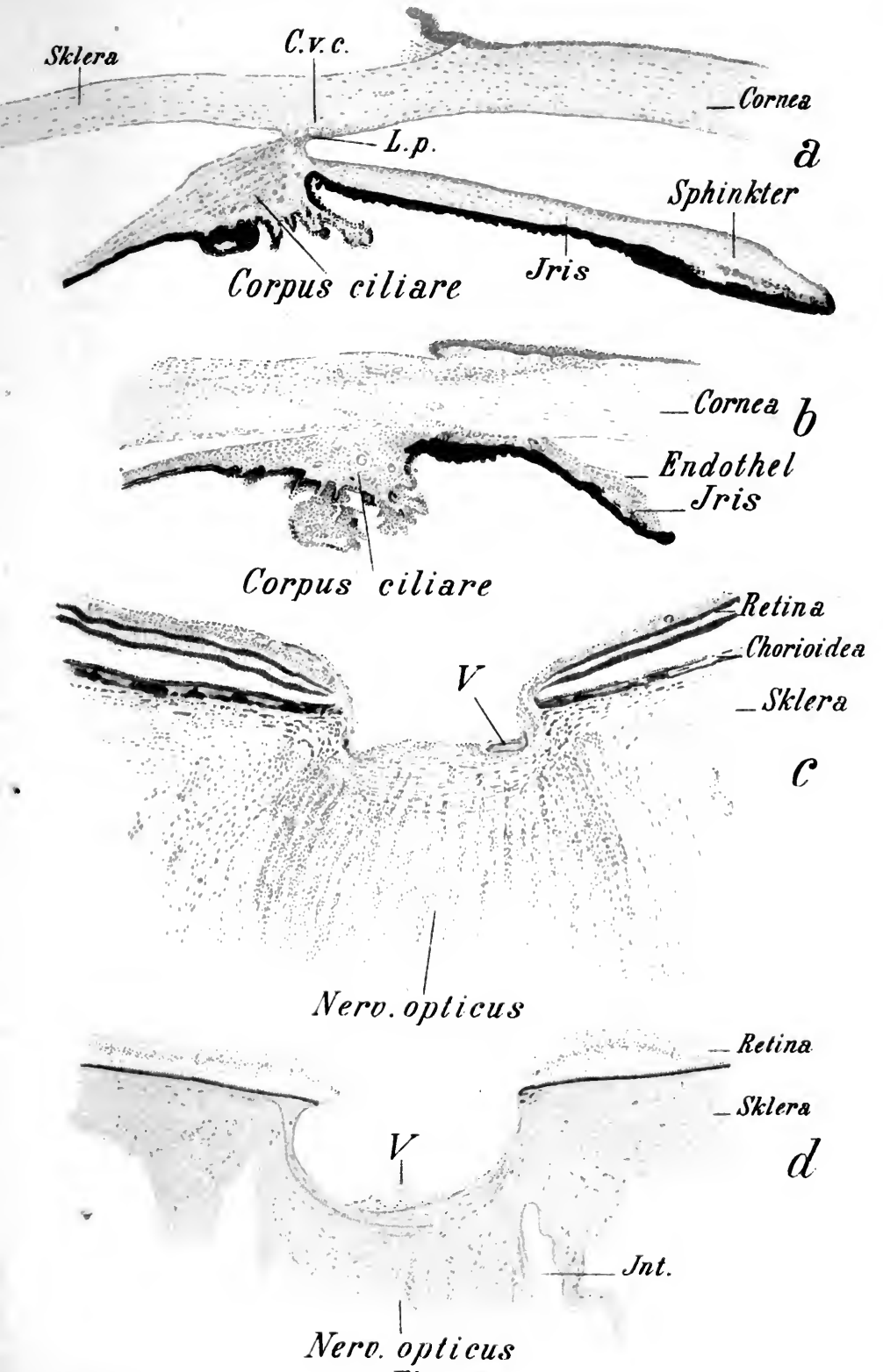

Fig. 24. 




$$
8
$$




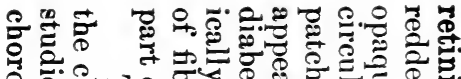

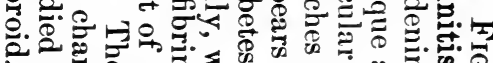

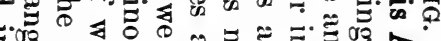

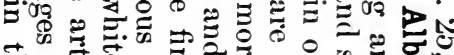

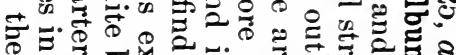

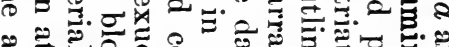
๘ ڤ की

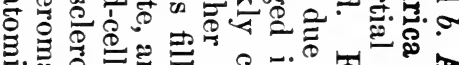

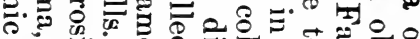

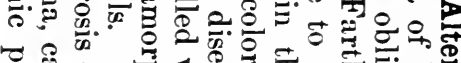

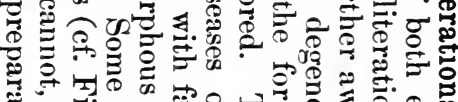

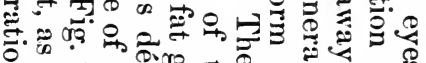

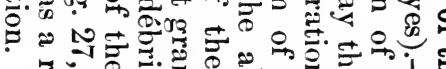

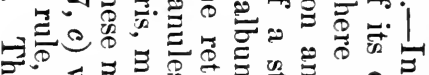

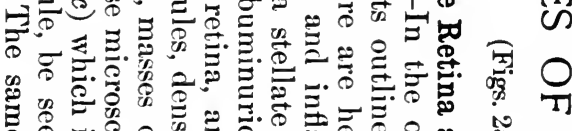

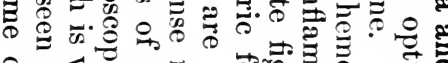

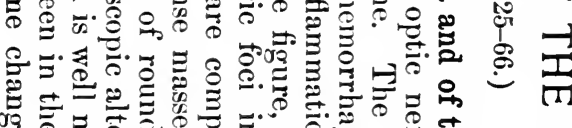

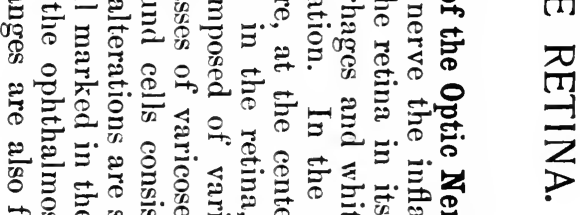

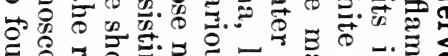

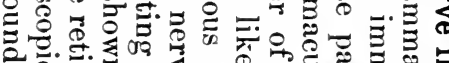

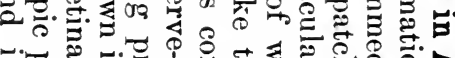

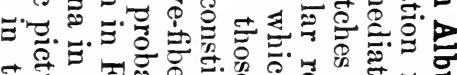

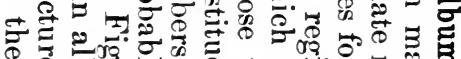
\%

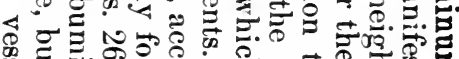
密

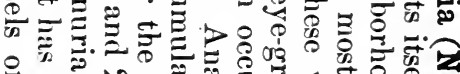

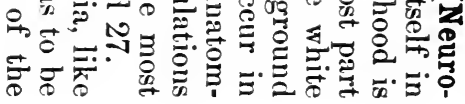


Fig. 26, a. Section through the Retina in Retinitis Albr. minurica.-Stained with hematoxylin and eosin.

The ravages produced by the disease will be at once seen by comparison with Fig. 3 . Numerous hemorrhages stained red by the eosin occupy the anterior layers of the retina. In the internuclear (outer reticular) layer we find between the fibers numerous gaps formed by edema and exudation. In one spot (stained very dark) is seen a mass consisting probably of fibrinous exudate (cf. Fig. 27).

Similar gaps are seen in the nerve-fiber layer which contains numerous varicose nerve-fibers shown under a higher power in Fig. $26, b$ and $c$.

Magnified 20 times.

The section corresponds approximately to the ophthalmoscopic pictures shown in Fig. 28, $a$ and $b$. The white foci in the retina correspond anatomically to the masses of fibrinous exudate represented in Figs. 26 and 28, or to varicose nervefibers that are often aggregated in bundles, or possibly to collections of wandering leukocytes and tissue-cells in a more or less advanced stage of fatty degeneration. The fatty degeneration is not seen in the specimen, which, however, shows masses of emigrated lymph-corpuscles scattered here and there among the tissues.

FIG. 26, $b$ and c. Varicose Nerve-fibers from the section shown in Fig. 26, a, under a higher power--Along the spindle-shaped thickenings of the nerve-fibers (stained violet by hematoxylin) are found nuclei of lymph-corpuscles or inflammatory leukocytes.

Magnified 112 times. 
c
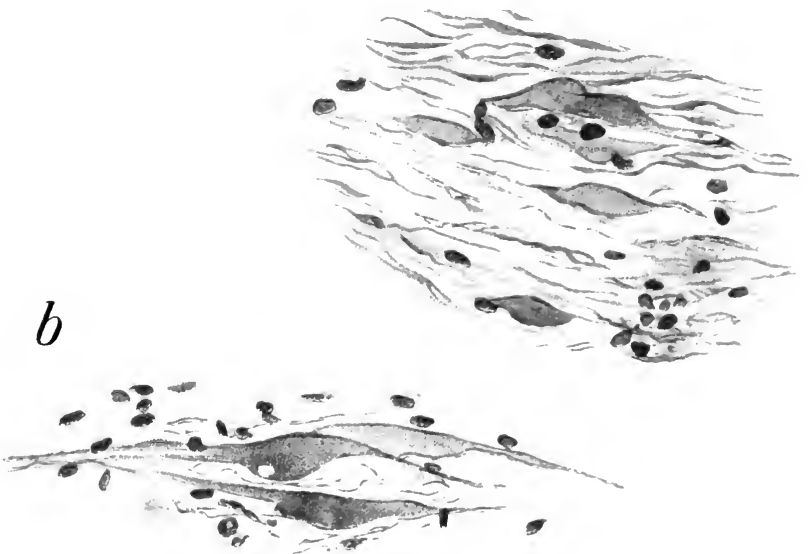

$a$

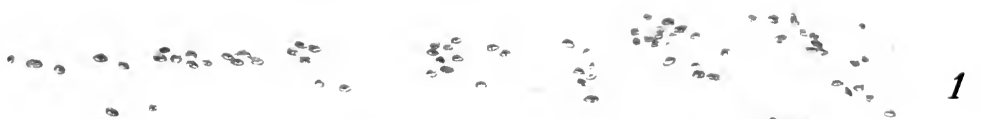

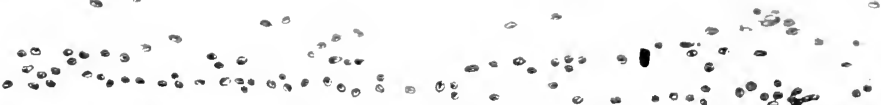

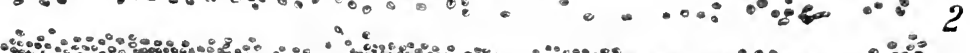

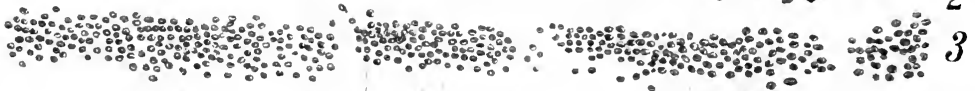

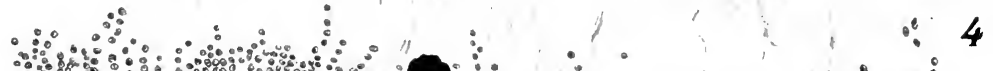
\%

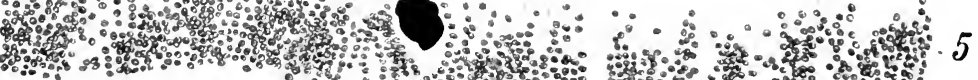
-

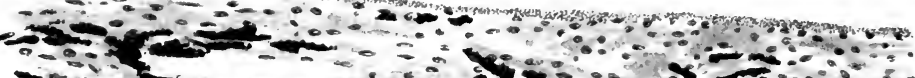

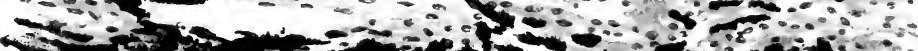

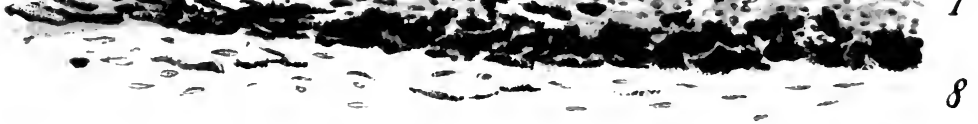

Fig. 26. 


M.l.i.

$a$
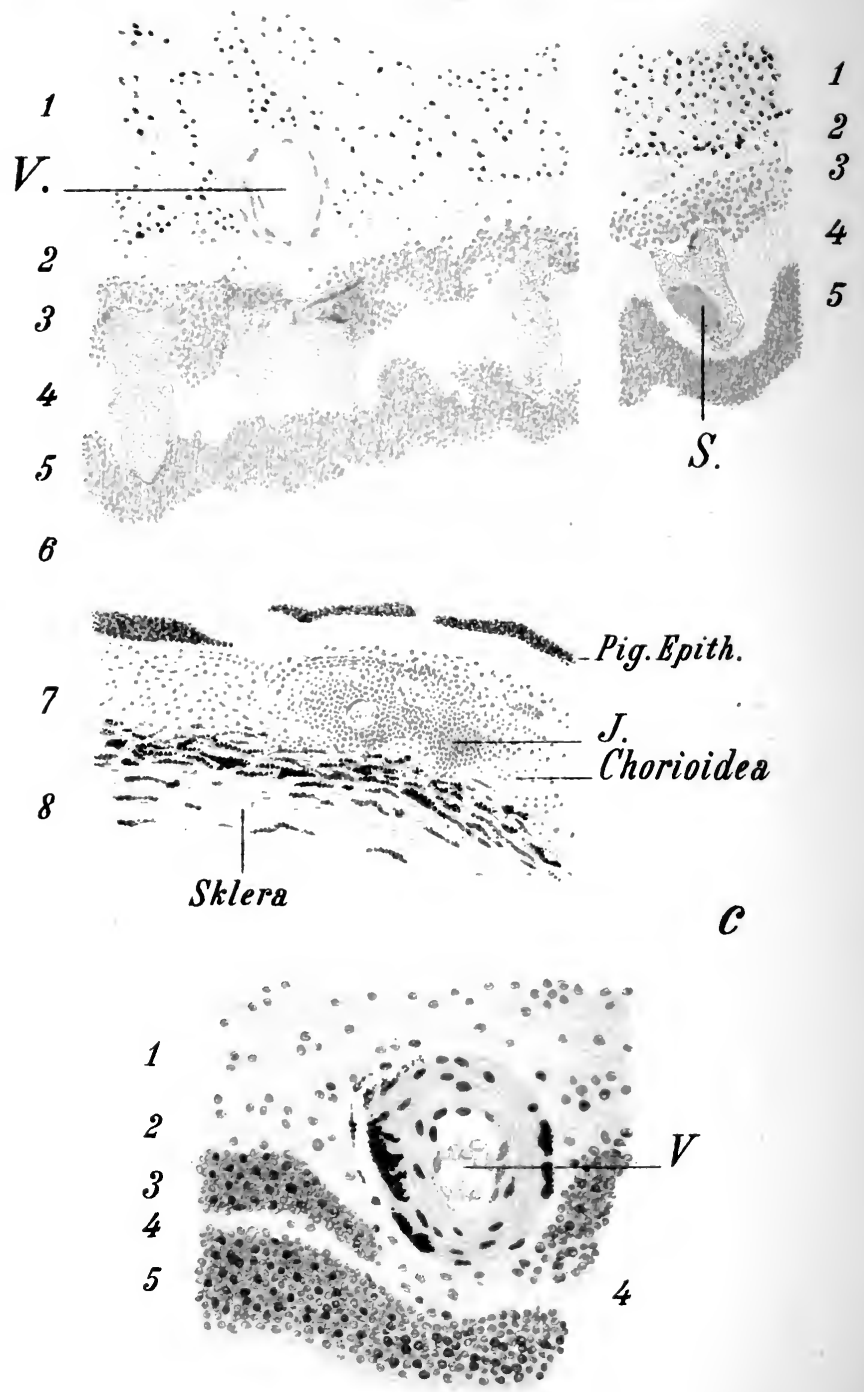

Fig. 27. 
FIG. 27. Alterations in the Retina in Retinitis Albuminurica.- $a$ represents a section through the retina, choroid, and part of the sclera. The interval between the layer of rods and cones and the pigment-epithelium, and the separation of the internal limiting membrane from the nerve-fiber layer are artefacts.

$b$ represents an adjoining portion of the same specimen. In the nerve-fiber layer and in the ganglion-cell layer leukocytes are seen scattered through the tissues, especially in Fig. 27, $b$. The lymph-spaces are wider than normal, owing to edema and the presence of amorphous exudate. The internuclear (outer reticular) layer contains a network of tough fibrinous exudate stained red by eosin. In Fig. 27, $b$, an amorphous fragment, similar to that shown in Fig. 26, is seen in the midst of the fibrin. The tissues of the internuclear (outer reticular) layer are likewise edematous (larger gaps in the tissue). The layer of rods and cones is degenerated and granular, the elements being widely scattered in places. The choroid is the seat of a pronounced inflammatory infiltration $(J$.$) . The blood-vessel V$., in Fig. 27, $a$, shows very little inflammatory thickening, in marked contrast to the blood-vessel $V$., in Fig. 27, $c$, the walls of which are greatly increased in thickness and contain pigment-granules.

1, Layer of nerve-fibers and ganglion-cells; 2 , inner reticular layer; 3, inner nuclear layer; 4, internuclear (outer reticular) layer; 5 , outer nuclear layer; 6 , layer of rods and cones; 7 , choroid ; 8 , sclera.

$a$ and $b$ magnified 122 times; $c, 150$ times. 


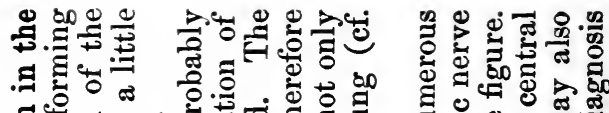

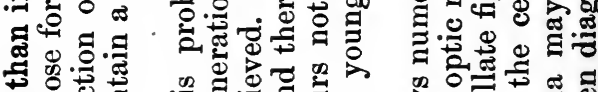

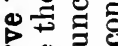

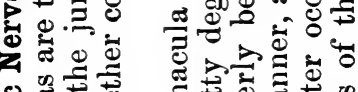

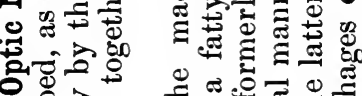

$\stackrel{乛}{+}$

을

๘

జ

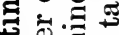

皮 800

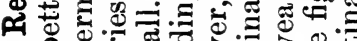

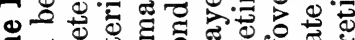

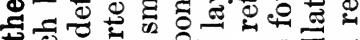

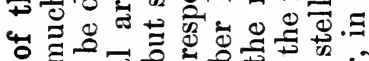

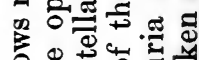

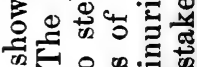

ํ.

.

过 윤

리를

평

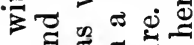

|.

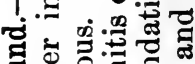

(3)

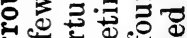
on

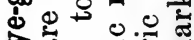

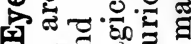

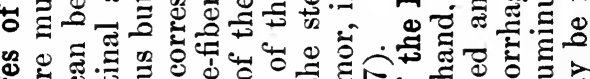

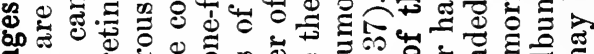

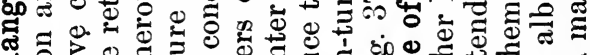

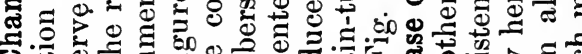

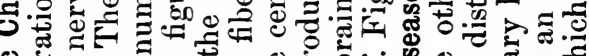

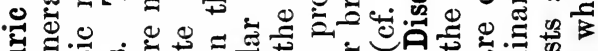

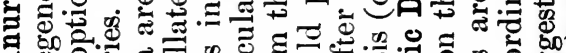
马 $00 \%$ 잉

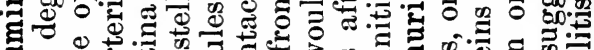

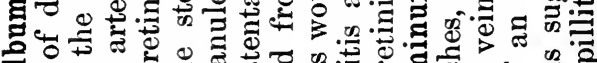
-

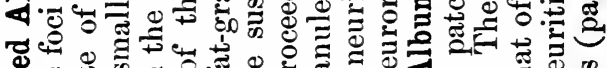
峲.

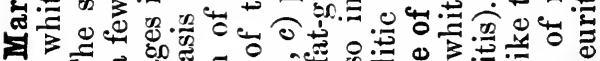

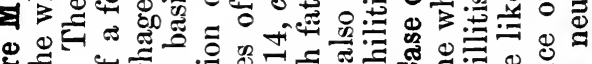
岁

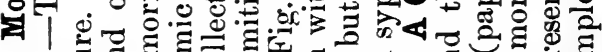

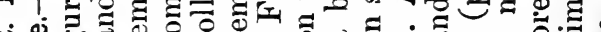
< \% $\infty$ తో 20 ॠ ஸे

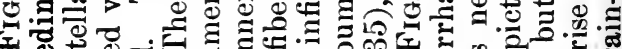

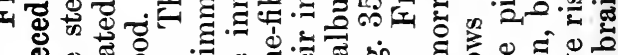

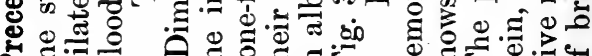
中清年 


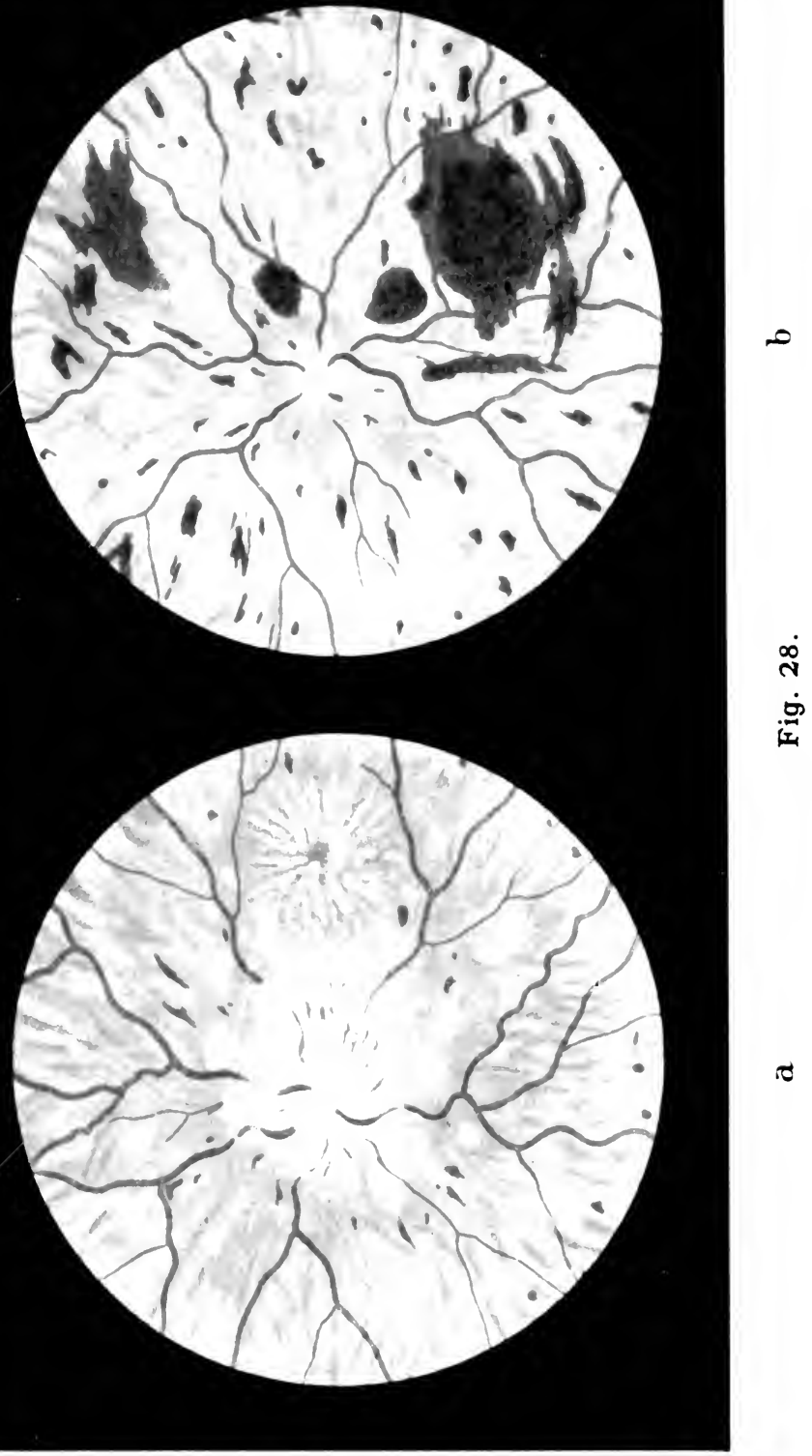






$$
8
$$




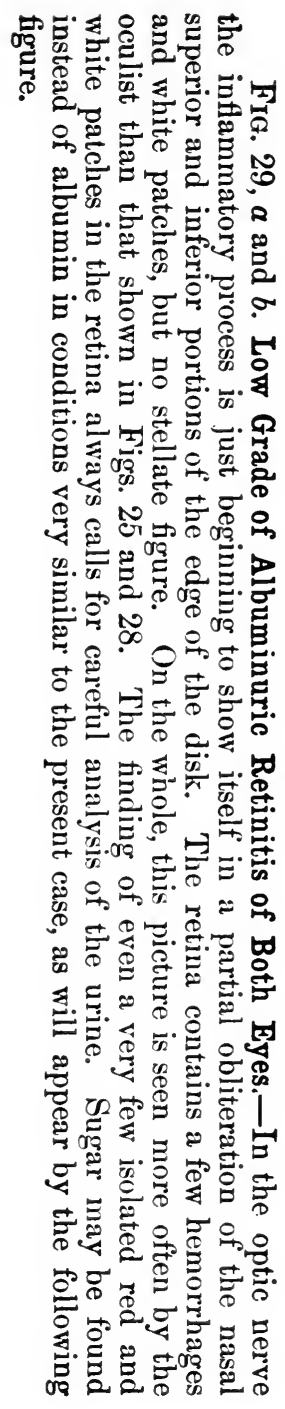




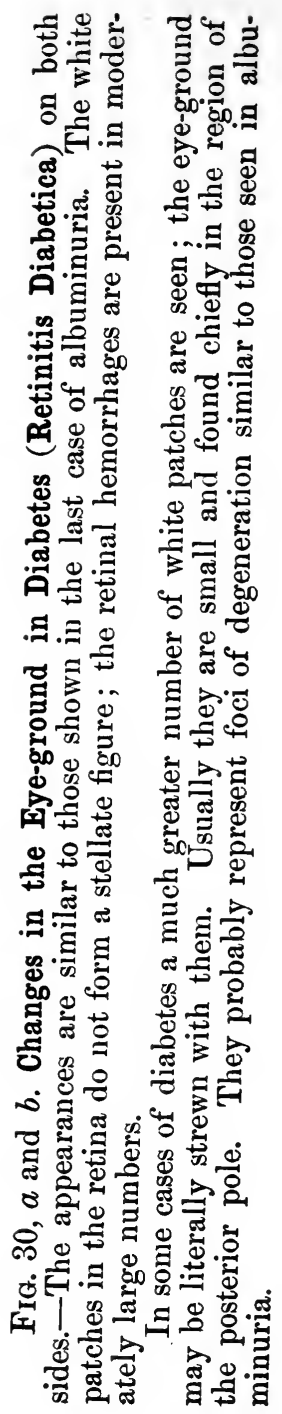




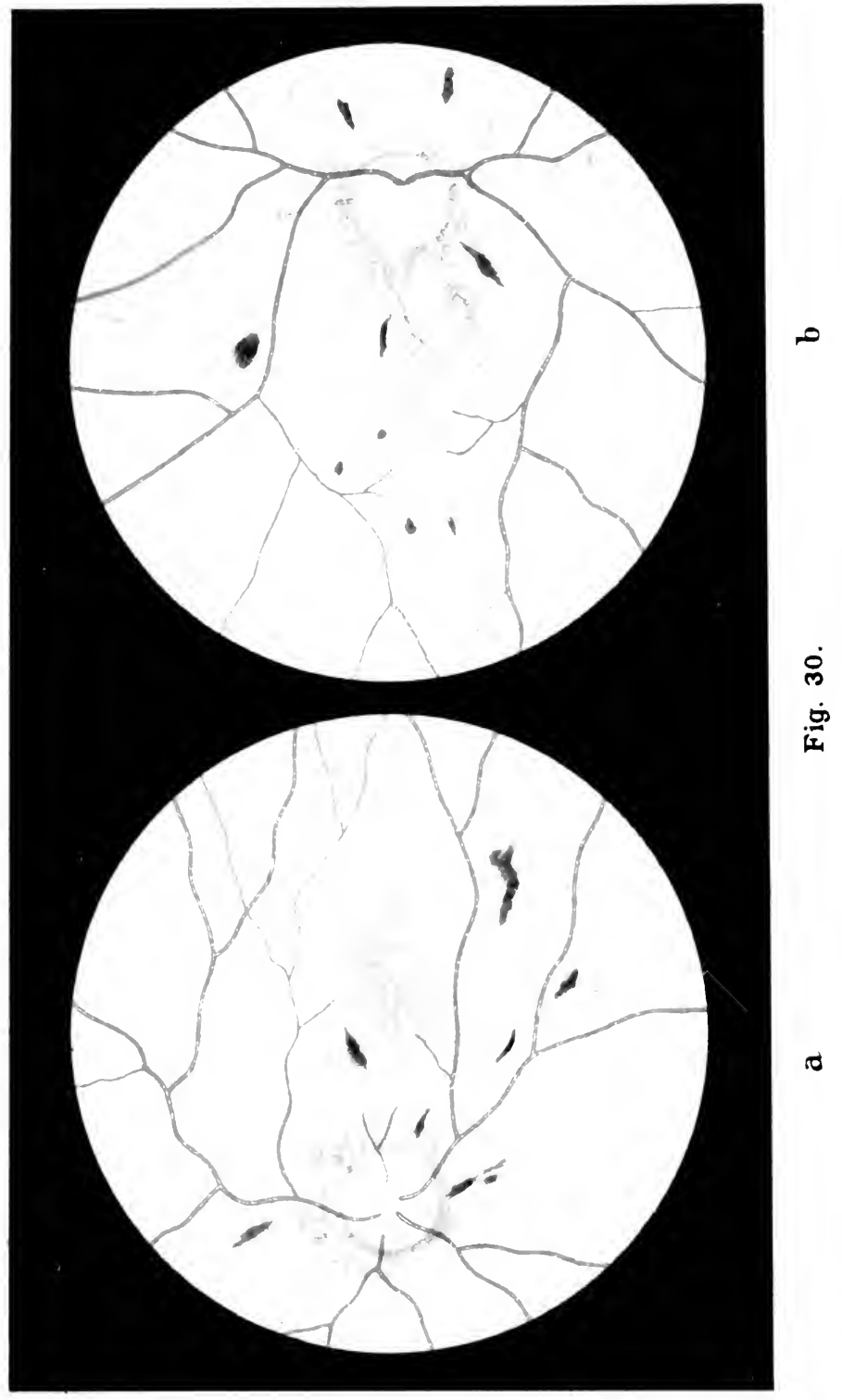


W.W. (2) $\because \because \frac{10}{3}=$

$n$.

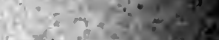
$\therefore i x^{2}+1-30$

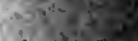

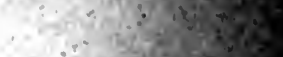

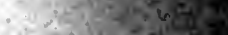

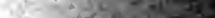

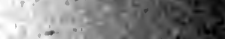
2. is

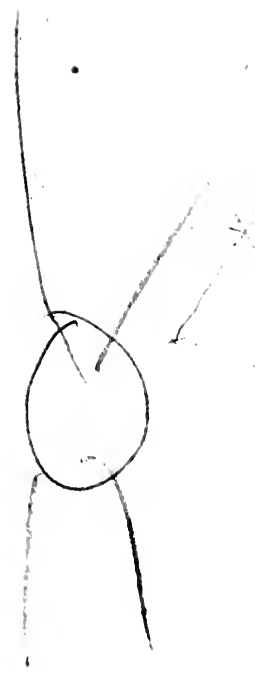

.

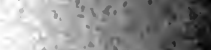

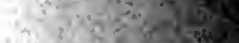

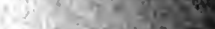
$4 x^{2}+7+2 n^{2}=$

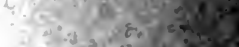

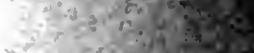

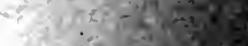

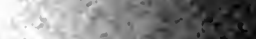

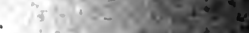

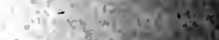

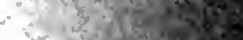

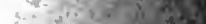

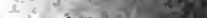

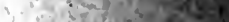

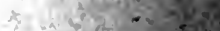

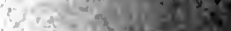

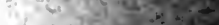
$\operatorname{tin}^{2}+1$

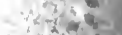

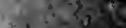

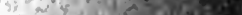

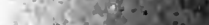

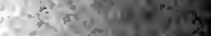
Mix? $y=2, y+3$

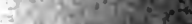

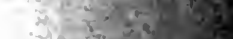

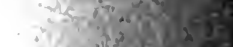

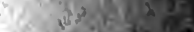

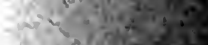

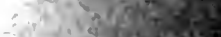

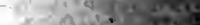
4hath

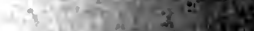

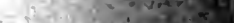

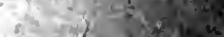

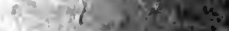

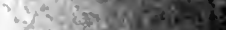

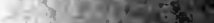

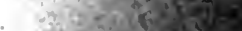

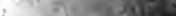

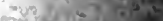

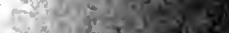

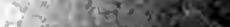

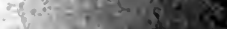

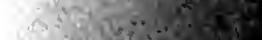

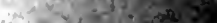

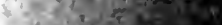

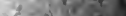

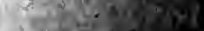

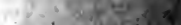





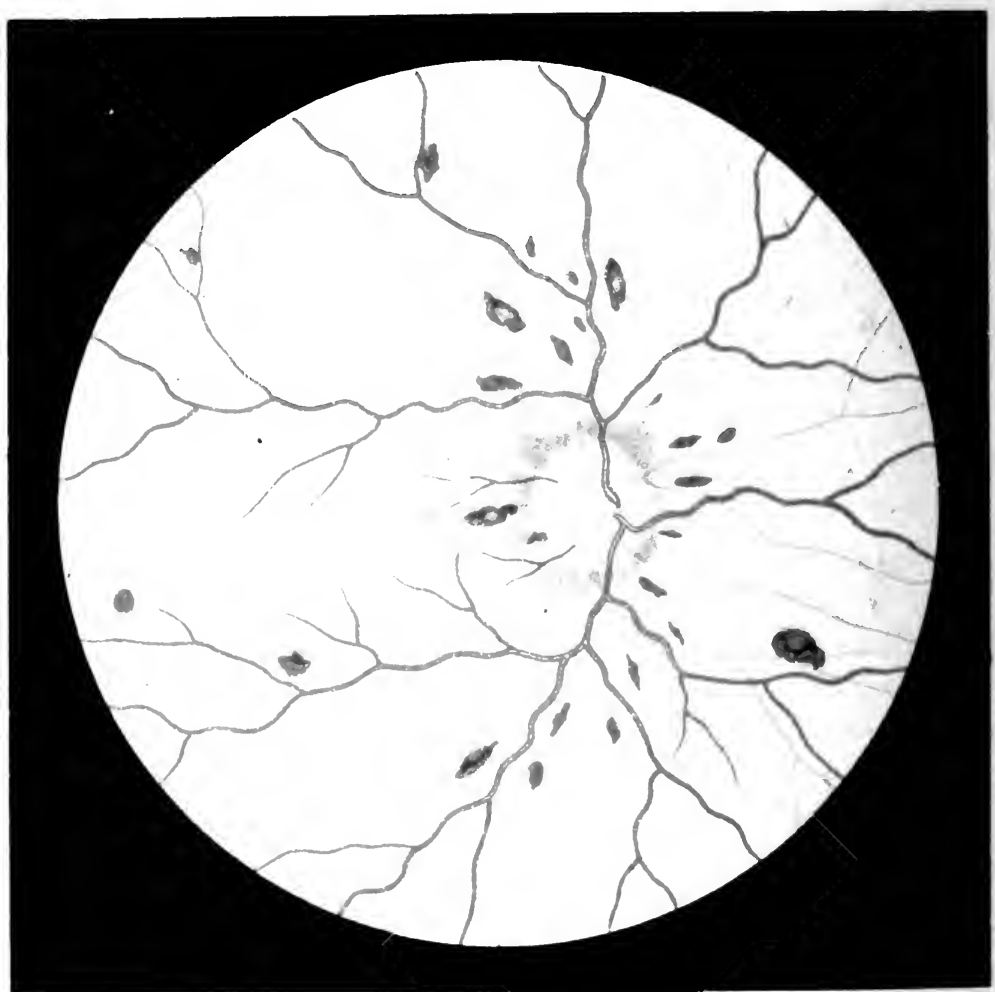

Fig. 31. 
Fig. 31. Changes in the Eye-ground in Pernicious Anemia.-The eye-ground is paler than normal. The disk is anemic and considerably lighter in color. The dilatation of the arteries is slight, that of the veins marked. The retina contains numerous hemorrhages; some of them are pale in the center, which is rather characteristic of pernicious anemia, although it also occurs in other forms of retinal hemorrhages. A few white patches are also seen near the optic nerve. The patient, who belonged to Prof. Eichhorst's clinic, died soon after the picture of the eye-ground was made.

Fig. 67 shows a microscopic section of an area of the retina containing hemorrhages of this kind. 


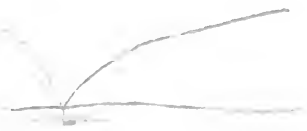

Fig. 32. Obstruction of the Central Artery.-The edges of the optic disk are obscured by a thick white opacity of the retina, which extends over the entire region of the posterior pole. The vessels in the neighborhood of the optic nerve are indistinct in places as if they were interrupted, while in the macular region the minute retinal vessels appear especially distinct. In the middle of the foveal region we see a cherry-red circular patch, not due to hemorrhage, but to a thinning of the retina, allowing the choroid to be seen through it, the red color being intensified by contrast with the white surroundings. The retinal arteries, which at first were contracted, have now regained their normal caliber fairly well, but they are still narrower than the veins. The blood-column in the arteries is interrupted in places. At the periphery the retina is normally transparent, so that the pigmented intervascular spaces of the choroid are plainly seen.

The obstruction of the central artery, which is the cause of this ophthalmoscopic image, was formerly always attributed to embolism. In my opinion the cause of the circulatory disturbance is much oftener thrombosis or obliterating endarteritis, embolism being very rare. 


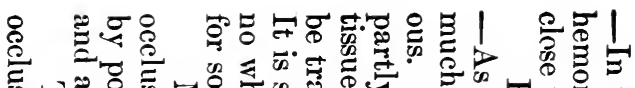

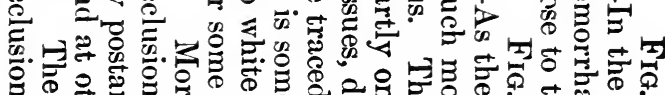
50

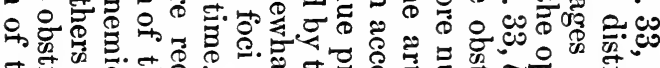

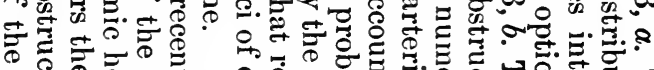

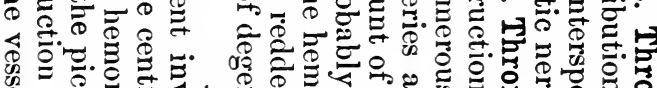

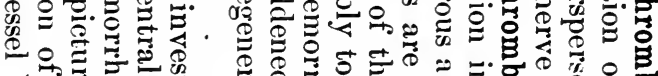

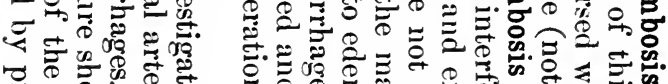

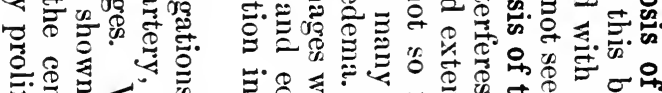

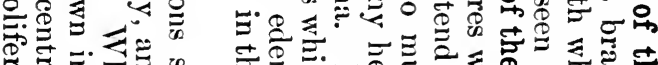

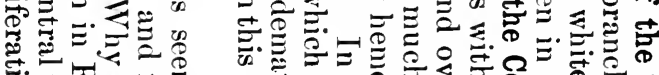

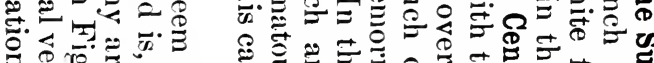

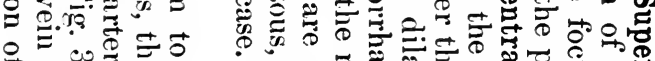

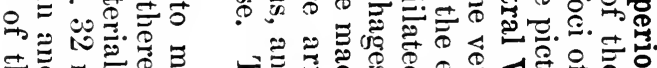

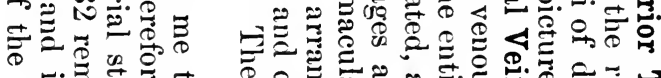
它语.

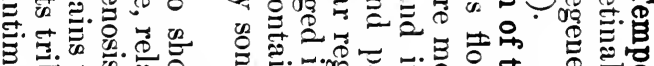

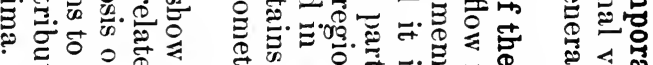
为 .

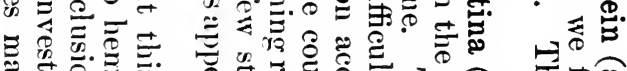

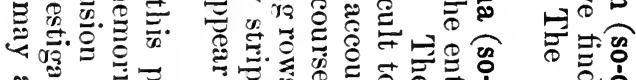

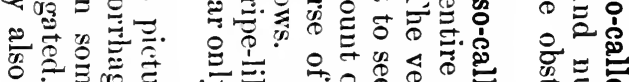

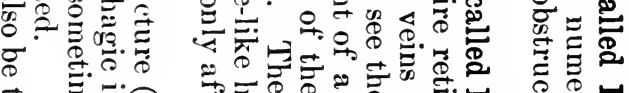

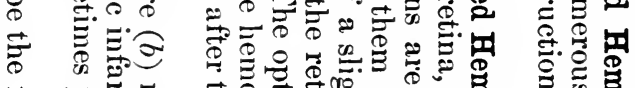

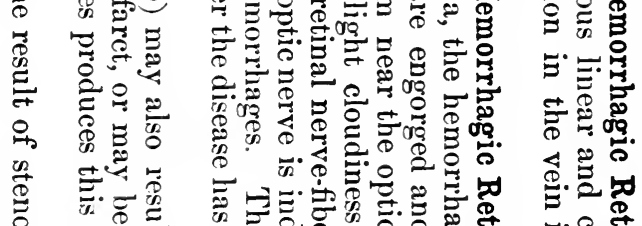

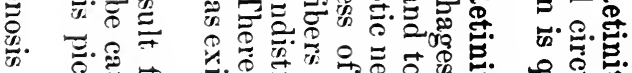

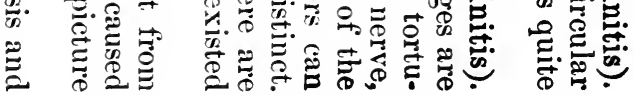


FIG. 34. Obstruction of the Superior Temporal Artery of the Retina by Thrombosis, Obliterating Endarteritis, or Embolism.-The retina is cloudy in the distribution of the arterial branch. The site of the obstruction carinot be clearly seen. The obstructed vessel contains nearly as much blood as a normal vessel. The opacity of the retina is most sharply defined toward the macula, where it is also most intense, as is also usually the case in obstruction of the central artery.

The condition was found in an old man who was suffering from arterial sclerosis and later died of cerebral apoplexy. 


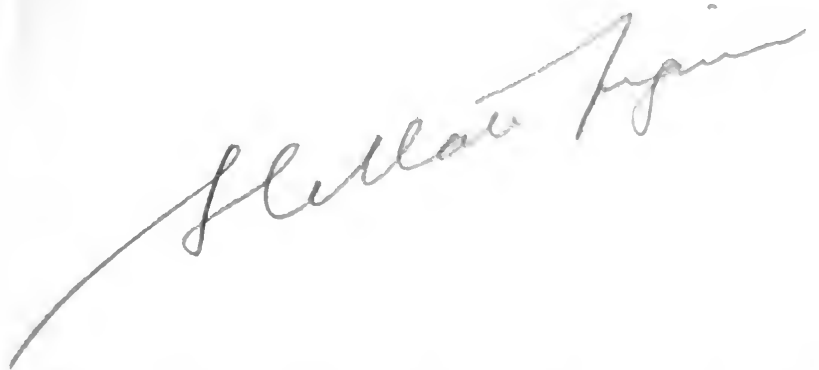

Fig. 35. Recurring Hemorrhages in the Retina and Vitreous in a Young Individual (direct method).-Later, hemorrhage into the vitreous also occurred and it became very difficult to see the eye-ground. The cause of the process was never determined. The urine contained no albumin, hence the stellate figure in the macula cannot be attributed to albuminuria. A vein in the nasal portion presents white markings. The retina contains numerous hemorrhages of various sizes. A large hemorrhage above the posterior pole, which does not alter its form, has sent blood down to the macular region where the blood, being fluid, forms a horizontal layer. When the head is inclined to one side the level of this collection of blood immediately assumes the corresponding angle, showing that we have to deal with a sacculated hemorrhage between the vitreous and the retina.

These preretinal hemorrhages are often situated immediately in front of the fovea of the retina and usually produce a small round reflex, which absolutely resembles the small fovea reflex. In my opinion the reflex may be explained as follows : the portion of the vitreous lying in front of the retinal fovea-representing a cast of the retinal fovea-retains its shape and dips down into the hemorrhage in the same way as it dips down normally into the fovea. Thus, it forms a depression in the hemorrhage, which causes a reflex similar to that of the retinal fovea. 
FIG. 36. Syphilitic Disease of the Retinal Arteries.-In the lower left-hand portion of the picture, corresponding with the distribution of the diseased arterial trunk, the retina is cloudy and covered with numerous hemorrhages. The disease of the arterial walls manifests itself in various places in this region in the form of white lines which accompany the blood-column. In places the blood-column is entirely obscured by the walls which have become opaque. In the lower right-hand portion of the picture there are also a large number of hemorrhages, but the retina is not cloudy and no vascular changes are visible. At the opposite border of the picture (to the left and above) is seen an artery with changes in its walls without, in this case, either hemorrhages or opacity of the retina. The retina probably does not become cloudy unless the disease leads to complete occlusion of the vessels and cuts off all the blood-supply through the collaterals. Hemorrhages may be present even when the arterial disease cannot be detected with the ophthalmoscope. Finally, the walls of the arteries may appear white without either opacities or hemorrhages appearing in their distribution.

The pathology probably corresponds to that of syphilitic disease of the arteries in other parts of the body (brain, etc.): thickening of the intima and adventitia, first by cellular, and later by fibrous tissue. The thickening is often so great that the lumen of the arteries is almost or quite obliterated. 


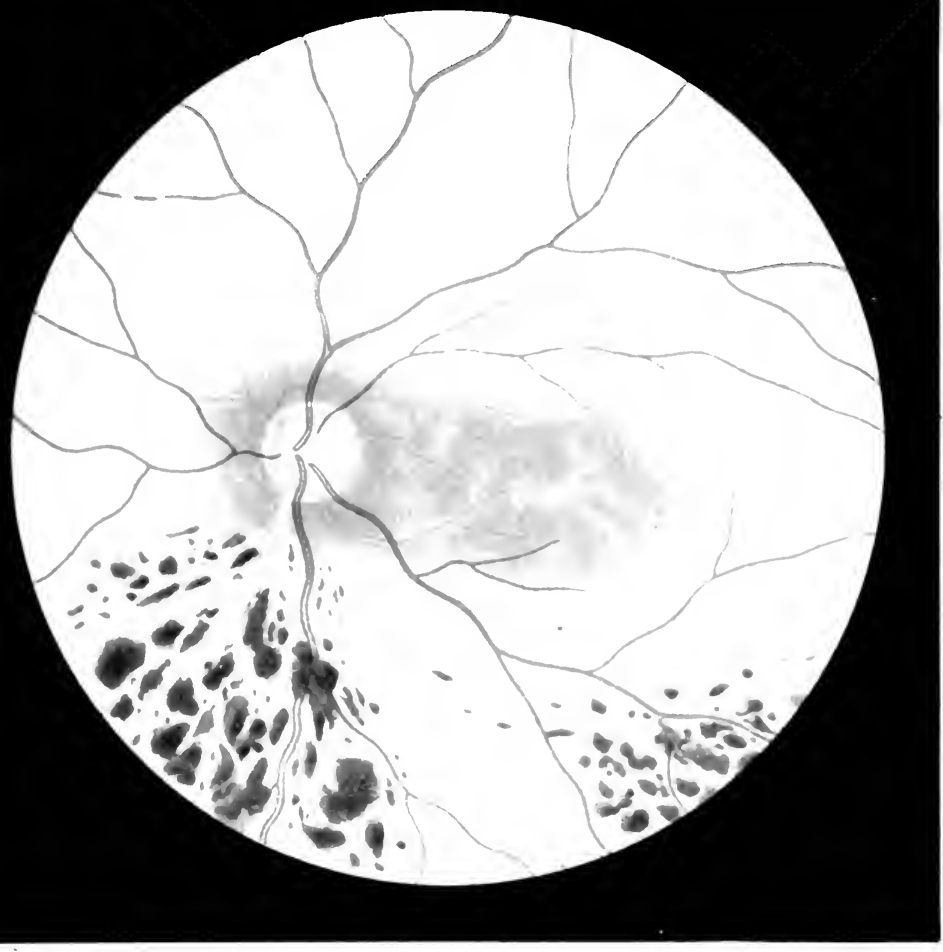

Fig. 36. 



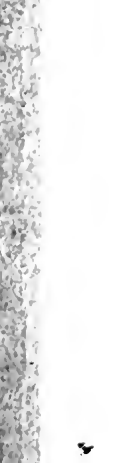




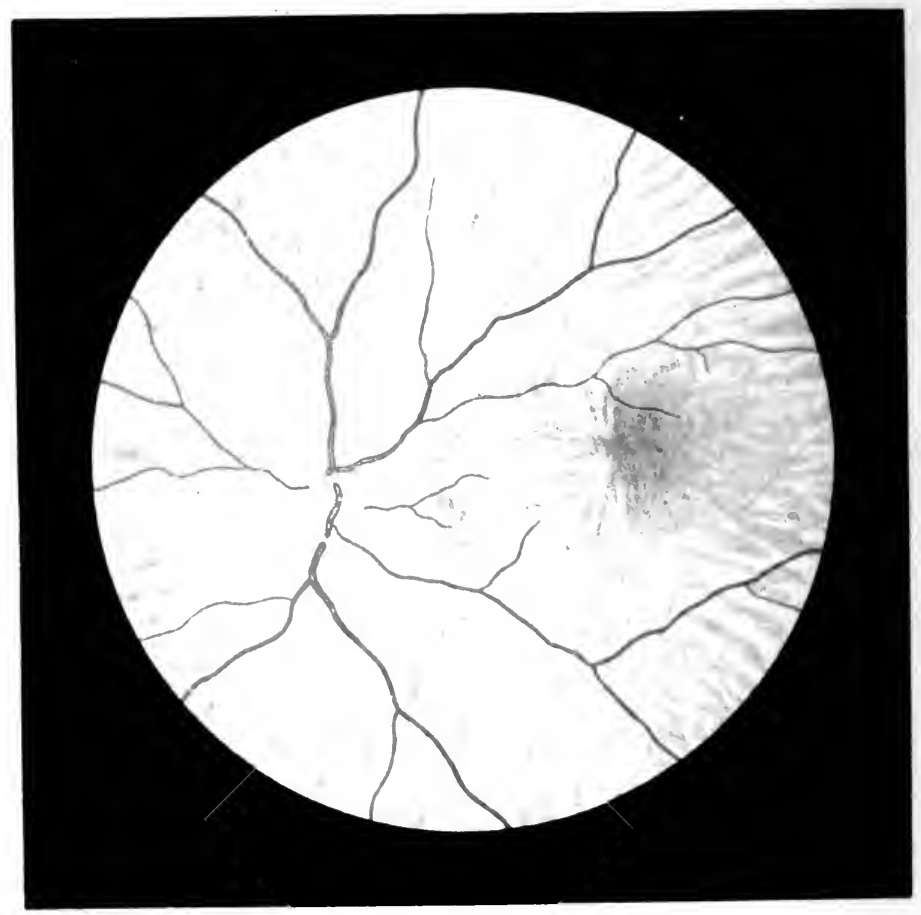

Fig. 37. 
FIG. 37. Syphilitic Neuroretinitis and Disease of the Retinal Arteries, Fourteen Days after the Beginning of the Disease.-The onset was sudden; one eye only was affected. At first there was merely the picture of neuroretinitis with marked hyperemia (corresponding approximately with Fig. $12, a)$, and in the macula a diffuse opacity with a small, round, grayish patch. Vision was completely lost in the center, in the entire nasal half, and in the upper outer portion of the visual field. In spite of immediate and energetic treatment vision did not return and soon the picture of Fig. 37 developed. Although no albumin was ever found in the urine, numerous white foci of degeneration appeared in the retina near the macula and about the optic disk, while the inferior temporal artery showed syphilitic disease of its walls in the form of small white scales. At the same time the neuritis disappeared and was replaced by atrophy. Infection had occurred twenty-seven years before.

This disease of the retinal artery, manifesting itself in white scales, I consider peculiarly characteristic of syphilitic disease of the vessels.

This case also shows that the stellate figure in the macula is not peculiar to albuminuria. It is probably a general expression of intense disease of the vessels and corresponding interference with the retinal circulation. 
Fig. 37, a. Angioid Streaks in the Retina.-The patient, aged fifty, presents nothing important in his clinical history, and physical examination fails to demonstrate the presence of organic disease. One brother has angioid streaks very similar to those portrayed in the illustration.

$a$, Right Eye. $-V=$ counting fingers at $60 \mathrm{~cm}$. The optic disk preserves its capillarity upon the nasal side, but presents upon its temporal aspect a patch of atrophic color. It is surrounded by an area of superficial choroidal atrophy. The retinal vessels, both veins and arteries, are about normal in size. Directly in the macular region, and occupying a large space of the temporal half of the eye-ground, is a huge, almost circular hemorrhage, broader and more fringed below, its center being occupied by whitish and yellowish areas of retinochoroidal atrophy. Chiefly upon the nasal side, and generally following the course of the retinal vessels and lying in the plane beneath them, is a system of branching streaks, somewhat granular in appearance, partly brownish and partly reddish in color. Within the boundaries of the hemorrhage previously noted may be seen another system of streaks, some brownish, and others still fresh and hemorrhagic, and directly connected with and proceeding from the band of blood-extravasation which circles this area. Downward and inward from the margin of the hemorrhage may be seen a small patch of similar streaks just beginning to form. The rest of the eye-ground is without notable lesion.

$b$, Left Eye. $-V=$ counting fingers at $50 \mathrm{~cm}$. The optic disk is round, the atrophic area being more decided above than elsewhere. It is surrounded by a patch of superficial choroidal atrophy and pigment disturbance. Everywhere throughout the eye-ground are numerous anastomosing brownish and reddish-brown streaks, of a character similar to those found in the other eye, but evidently at a more advanced period of their formation. The macular region and temporal half of the eye-ground are occupied by a large vertically oval plaque of choroidal atrophy streaked with black pigment. Bordering this below, and between it and the disk are broad reddish bands, somewhat resembling exposed choroidal vessels, of which, in some instances, they are representations. It is evident that the process in the left, which is the one first affected, represents a further advanced stage than that in the right eye. 
$a$

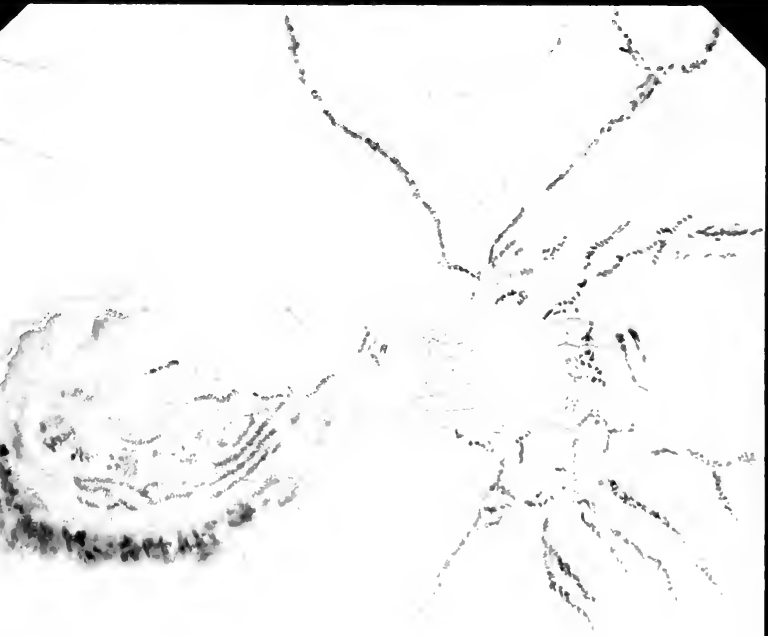

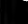

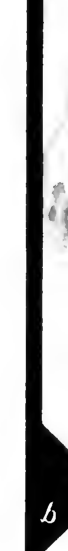
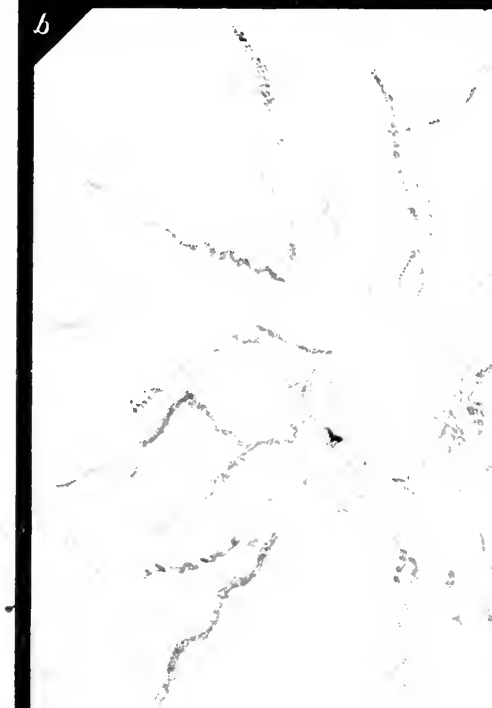


$$
\text { - }
$$

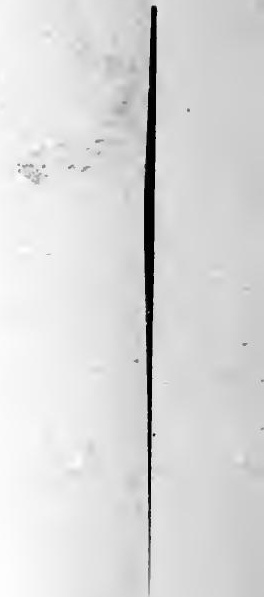


FIG. 37, $b$. Changes in the Eye-ground in Arteriosclerosis.The left eye-ground represented is that of a heavily built florid woman, aged sixty-five, who since 1890 suffered much from bronchitis of the influenza type. At night she is somewhat asthmatic and complains of being rheumatic; she is much troubled with drowsiness. The capillaries on her face are somewhat distended over the bridge of the nose and on the cheek-bones. The radial artery is a little rigid to the touch, and more rigid upon the left than upon the right side. The temporal arteries are plainly visible, although not greatly distended. The urine has a low specific gravity and contains a trace of aibumin and occasionally a hyaline cast.

The vision of the eye is $\frac{6}{6}$. Ophthalmoscopically, the following lesions are evident: Edema of the nasal margin of the disk: light-colored, somewhat tortuous arteries, the upper temporal artery being markedly and unevenly narrowed and constricted, and bordered by one or two old extravasations; distended veins, presenting numerous alternate contractions, typically exhibiting the signs of mechanic pressure where they are crossed by the diseased arteries, the upper temporal vein being so impeded in its circulation that it is fringed with a white border of infiltration, unevenly contracted and gradually dwindles to a tortuous thread, preceded by several varicosities; in other words, in one eye-ground, all of the appearances which are characteristic of arteriosclerosis.

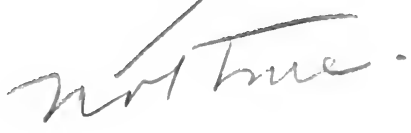


Fig. 38. Pigmentary Degeneration of the Retina (Retinitis Pigmentosa).-The disease always affects both eyes. The degenerative element in the disease is much more marked than the inflammatory, hence the term pigmentary degeneration is to be preferred. The degenerative nature of the disease manifests itself in the contraction of the vessels which appears very early [in the disease]. As the process goes on, the arteries and veins become smaller and smaller. At the same time the optic nerve gradually assumes a slightly atrophic, yellowish-white appearance. The entire eye-ground becomes päler, more gray or leaden-hued, the longer the disease lasts. The characteristic coal-black pigmented patches first appear singly in the periphery of the retina and slowly increase in size as the years go on. (The picture shows quite an advanced stage.) The pigment-patches are always small, sharply outlined, with serrated edges resembling bone-corpuscles, or stellate, and occasionally linear or bifurcated; they follow the course of the retinal vessels. The macular region and the region of the optic nerve remain free from pigment-patches longer than any other part of the eye-ground. White patches do not occur. In exceptional cases a few yellowish-white atrophic foci may later develop in the choroid, usually very near the periphery, or there may be a sprinkling of whitish dots in the macular region. Later, sclerosis of the choroidal vessels not infrequently becomes visible, the vessels assuming first a yellowish, then a whitish color (see Fig. 81). The yellow coloration of the choroid vessels is seen above the optic nerve in the illustration. 
$$
\text { - }
$$

$$
\text { . }
$$




\section{Fig. 39. Pigmentary Degeneration of the Retina (Retin-}

itis Pigmentosa), Advanced Stage.-The color of the eyeground is more gray or lead-colored, the pigment-patches are thicker, and in places form a regular network. The atrophic discoloration of the optic nerve is more marked and the vessels are smaller than in the last picture. This case illustrates the peculiar character of the visual disturbance in this disease. The visual field becomes smaller and smaller and is finally reduced to a minimum as the disease progresses. As shown in the figure, the outermost zone of the retina remains free from pigmentation until this time. Accordingly, the defect in the visual field was a circular scotoma corresponding to the zone of pigmentation of the retina, showing that the retina loses its function wherever it is attacked by the disease. The pigmentation is probably a secondary process, as contraction of the visual field may occur without it.

As the disease progresses the pigmentation spreads centrifugally and peripheral vision is also destroyed ; while, on the other hand, the centripetal advance of the process causes contraction of the central visual field.

The anatomic changes of the later stage are shown in Fig. 46, $c$ and $d$, where the enormous atrophy of the entire retina is much more conspicuous than in the ophthalmoscopic image. The section shows how the retina eventually becomes entirely converted into connective tissue and loses its normal transparency, explaining the gray appearance of the eyeground in the severer forms of this disease. 
Fig. 40. Disease of the Eye-ground in Hereditary Syphilis. -This figure and the two following represent various forms of the same disease-hereditary syphilis-which, like the specific process in general, manifests itself in various forms. Whether the primary seat of the disease in these cases is in the retina (pigment-epithelium and rods and cones) or in the choroid, has not, in my opinion, been definitely established. I have inserted these pictures among the retinal diseases because the pigment-patches appear to me to be situated for the most part in the retina, and because in some cases the disease shows a certain similarity to pigmentary degeneration of the retina which has just been described. The periphery in the lower left-hand portion of the picture presents a leaden hue and coal-black circular and triangular pigment-patches. The rest of the eye-ground is covered with minute yellowish-red spots. The yellowish-red roundish spots stand out from the brown dotted background, which looks as if it were sprinkled with snuff. The retinal vessels are rather small and the optic nerve somewhat discolored. I have chosen for this picture a case in which the disease was very well marked. Sometimes the sprinkling is found only in the periphery of the eyeground, and is neither so well pronounced nor so extensive as in this picture. But whenever this sprinkling is at all well marked it indicates hereditary lues. A similar but much finer sprinkling of the eye-ground occurs in cases of insufficient pigmentation (in blondes). The latter, however, is, as a rule, seen only by the direct method, while the luetic can be seen by the indirect method. 
Frg. 41. Alterations of the Eye-ground in Congenital Syphilis.-In this form, which is not quite so severe as that shown in the last picture, the disease is often confined for a long time to the periphery. In this disease also the pigmentpatches are probably, for the most part, situated in the retina and are caused by a disease of the pigment-epithelium. I am unable to decide whether the pale linear and circular yellowish patches are situated in the choroid or in the pigmentepithelium. They may be situated in one or the other.

This and the following form of the disease are not rarely found after a diffuse interstitial keratitis if, after the cornea has become sufficiently cleared up, the periphery of the eyeground is carefully examined. In some cases the patches may be confined to one side, in others they may be scattered more or less over the entire eye-ground [and on both sides].

In the case here represented there had been a keratitis.

The increased pigmentation at the margin of the optic disk, seen in Figs. 40-42, may not be present even when there is a chorioretinitis, and Antonellis's supposition that this marginal pigmentation (choroidal ring) is a stigma of hereditary syphilis is not quite correct. It occurs also in normal eyes. 
FIg. 42. Alteration of the Eye-ground in Congenital Syphilis.-In place of the black and gray patches, which predominate both in size and number in the foregoing picture, we find in some cases only whitish, also circular, and often confluent patches. The larger of these are undoubtedly situated in the choroid. This is shown in the figure by the oval white patch traversed by a red vessel. It is a choroidal vessel, hence the choroidal tissue near the vessel is absent and the white of the sclera shows through. There is no doubt, however, that the pigment-epithelium of the retina has disappeared at the site of the patches, and is slightly increased about their margins, so that the margins appear somewhat darker than the surrounding tissue. This condition also followed an attack of parenchymatous keratitis.

Occasionally the forms shown in Figs. 41 and 42 are associated, resulting in a mixture of both dark and light circular confluent patches at the periphery, while sometimes, in addition, we find the form shown in Fig. 40-viz., minute red and yellow spots on a brown, mottled background, with a sprinkling of small pigment-patches.

In general, these three types of hereditary specific eyeground disease show a tendency to the formation of spherical foci, which coalesce and form irregular figures. 


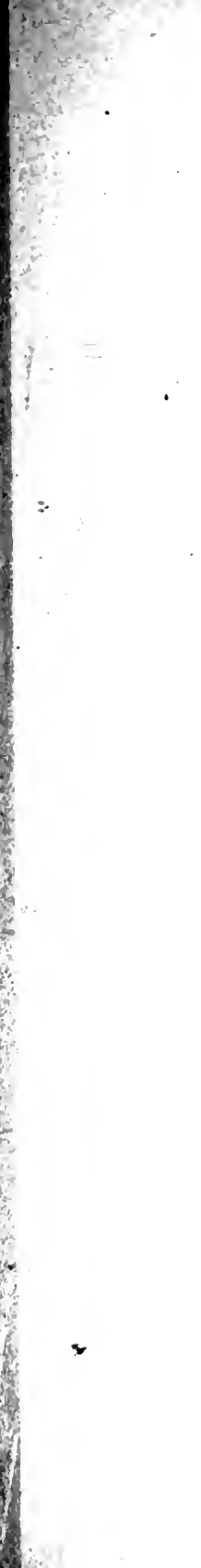




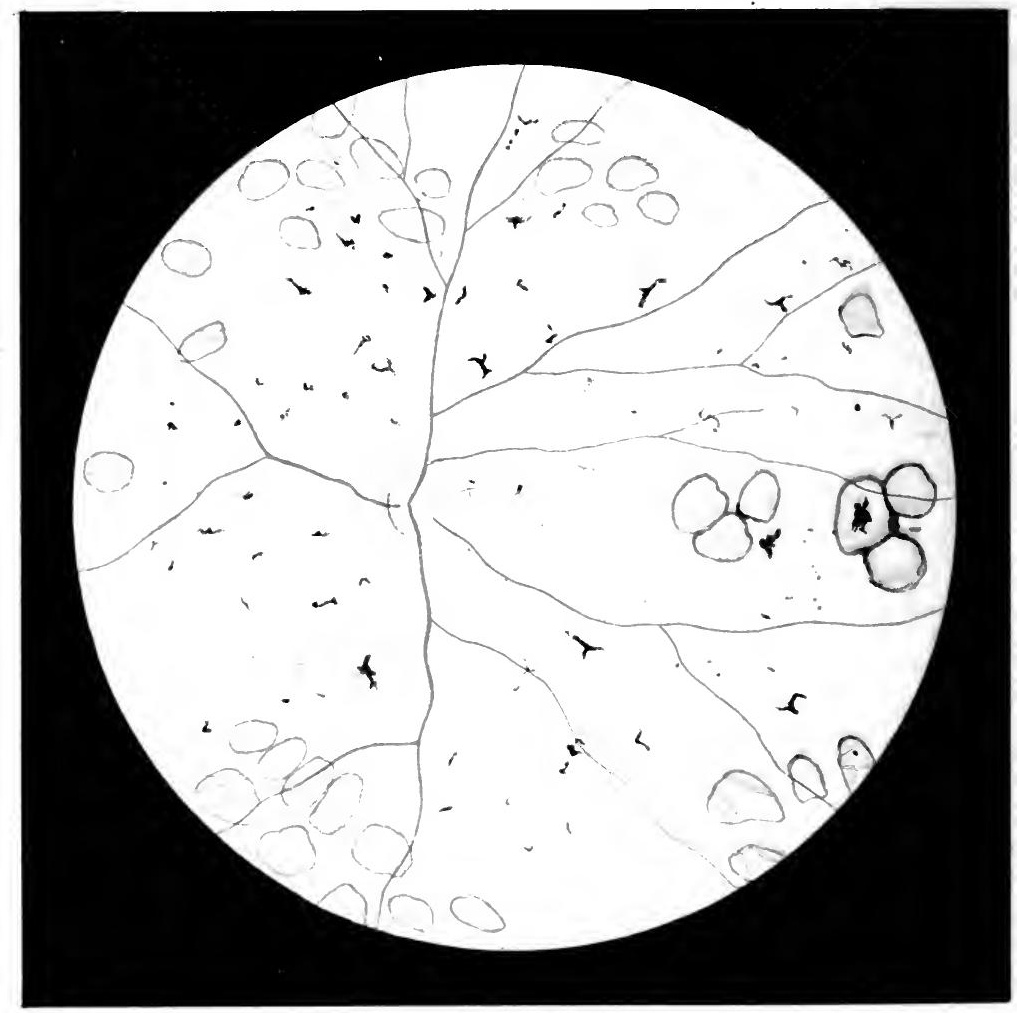

Fig. 43. 
Fig. 43. Secondary Pigmentation of the Retina in Disseminated Choroiditis. - In the central portion the picture somewhat resembles pigmentary degeneration (Figs. 38 and 39). The optic nerve is somewhat pale, the vessels are small, the eye-ground lighter than normal, with a suggestion of yellowish-gray. This area also contains pigment-patches exactly resembling those seen in pigmentary degeneration. In addition, however, there are large white circular, sharply outlined patches near the periphery. They correspond to portions of the sclera which have become visible, because both the pig. ment-epithelium of the retina and, owing to the disseminated foci of inflammation in the choroid-choroiditis disseminatathe choroidal tissues have disappeared. Nothing remains of the latter except here and there a few isolated choroidal vessels, traversing the patches like narrow red ribbons. And even the vessels are wanting in many of these atrophic foci of the choroid. One of them contains at its center a small patch of pigment. This variety of chorioretinitis, a later stage of which is here represented, is often complicated with more or less opacity of the vitreous, and is frequently caused by syphilis.

Secondary pigmentation of the retina also occurs after other forms of choroiditis if the inflammation is severe and lasts a long time, as will be shown in some of the later pictures. 


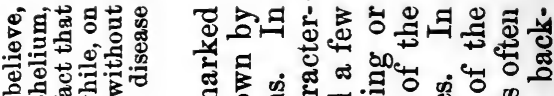

1.2. 3 o 政

if of o

.

政

ล.

2.

\% : 0

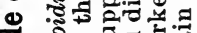

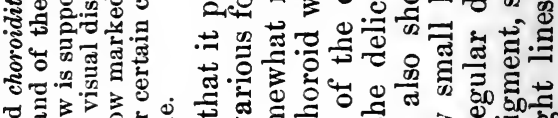

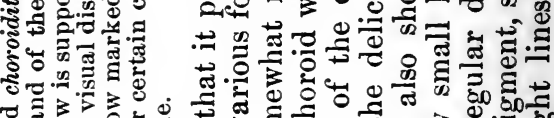

ถึ

届

क

$\circ$ :

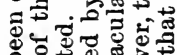

\&

t 0 .

๑ึ

蛋

要

0 .

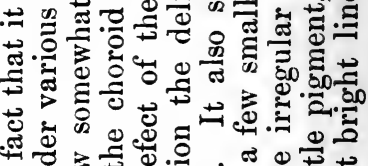

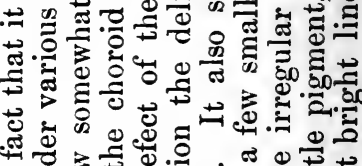
类 का का का 응

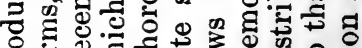

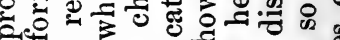

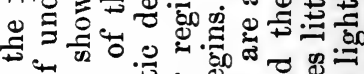

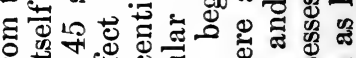
:

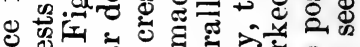

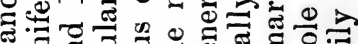

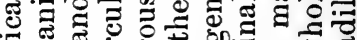
$\because]^{\circ}$ 50

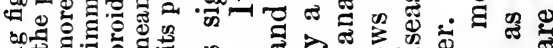
-

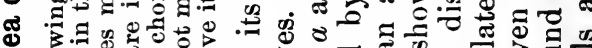

要

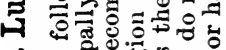

때

要

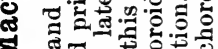

त.

\$

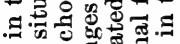

क 3 क

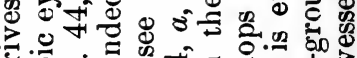
造. र्रा क्ष

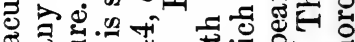
药

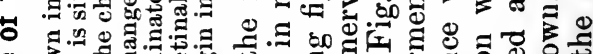

हैं

\% की \%

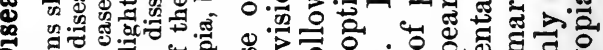

a

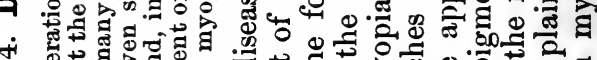
连

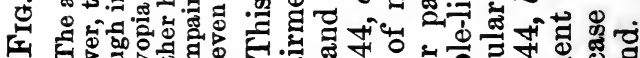

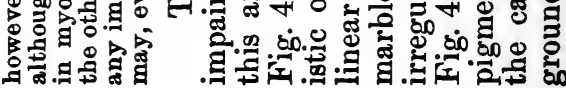




$$
8
$$




a
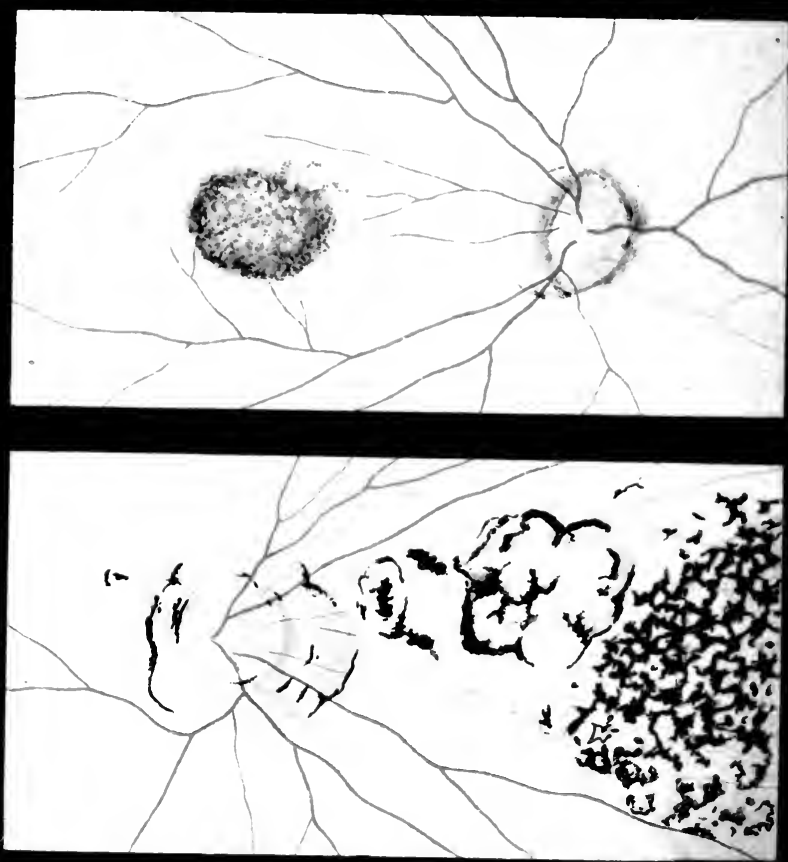

b

C

Fig. 45 . 
FIG. 45. Disease of the Macula Lutea of the Retina resulting from a High Grade of Myopia.-At $a$ one sees the reddening and slight swelling of the nasal portion of the nerve that are apt to develop when eyes of this kind are subjected to strain, and which probably represent a functional hyperemia, although they are regarded by some as inflammatory conditions. The disk also is somewhat oblique and surrounded by an atrophied portion of the choroid in the form of a "meniscus" or cone. The pigmentation of the eyeground is somewhat fainter than normal. The macular disease manifests itself in yellowish-red spots and a somewhat irregular pigmentation. The middle of the fovea is occupied by a black spot surrounded by small hemorrhages.

FIg. 45, b, represents a more advanced stage of the disease, recognized by an increase in the pigmentation and the presence of white spots, which indicate that the choroid is involved and atrophied in places, exposing the sclera. A few pale spots extend as far as the atrophic meniscus about the optic nerve.

Fig. 45, $c$, shows an advanced case of macular disease, in which there is not the slightest doubt of the choroid being involved. The optic nerve is surrounded by an atrophic ring which is broadest on the side near the macula. The macular region is occupied by a large white patch with sinuous and partly pigmented outlines within which the choroid has entirely disappeared, except for a few vessels and traces of pigment. To the temporal side of this the choroid and retina have disappeared, leaving a large atrophic patch extending as far as the equator, covered with irregular masses of pigment. 
Frg. 46. $a$. Secondary Pigmentation of the Retina caused by a Fragment of Percussion-cap (not shown in the picture), which remained in the eye twenty years.-Posterior segment of the eye seen from the front. At the center one sees the optic nerve and the retinal vessels radiating in all directions. In the left half of the figure the retina is detached from its base, but whether this condition was present during the individual's life, or is an artificial product, cannot be determined. The area of retinal detachment shows the pigmentation which closely resembles that seen in pigmentary degeneration (retinitis pigmentosa, see Figs. 38 and 39) better than the rest of the eye-ground. This preparation shows more advanced changes in the retina than are seen in the ophthalmoscopic picture in Fig. 74, which also shows the changes produced in the eye by a piece of percussion-cap. The patient, who was recently examined, now shows much more marked pigmentation of the retina than at the time this picture was taken.

FIG. 46, $b$. Sagittal Section of an Eye with a Total or Funnel-shaped Retinal Detachment of Long Standing.-The retina extends forward in the form of a band, which is broader in front, still contains some remains of the degenerated vitreous body, and surrounds the lens. The interval between the retina and the choroid is occupied by an amorphous exudate.

FIg. 46, c. Pigmentary Degeneration of the Retina (Retinitis Pigmentosa).- -This picture represents an advanced stage of the disease, hence the normal structure of the retina is entirely lost, being represented by a fibrous membrane containing many nuclei interspersed with pigment. 'The rods and cones have disappeared completely. The choroid is normal.

Magnified 30 times.

Fig.46, $d$. A Portion of the Same Specimen under a Higher Power.-In places the pigment is collected about the bloodvessels in accordance with what we see in the ophthalmoscopic image: P.P., pigment in the retina.

Magnified 78 times. 

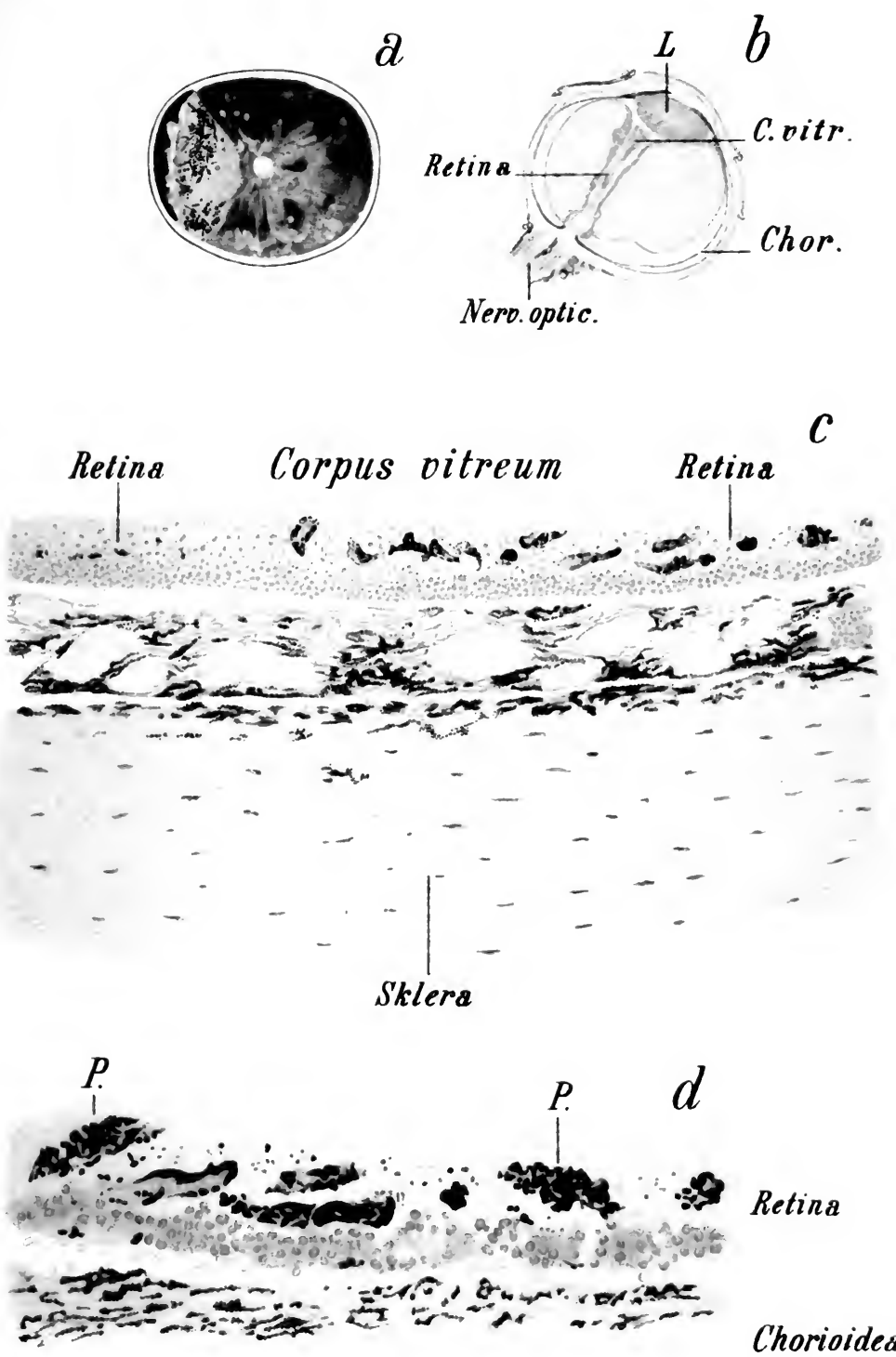

Chorioidea

Fig. 46. 



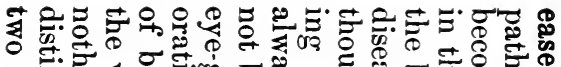

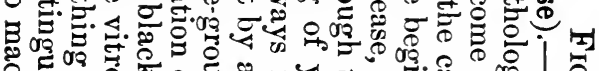

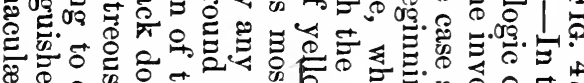

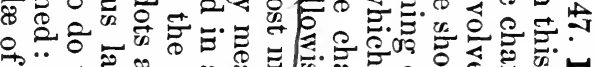
ڤ

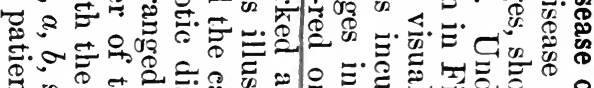

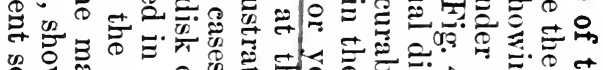
क

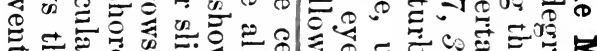

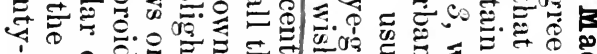
家 引

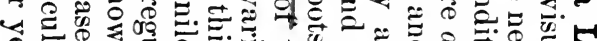

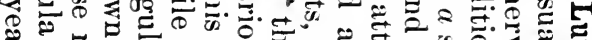

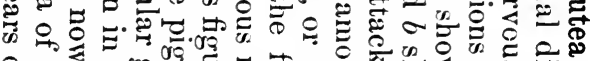

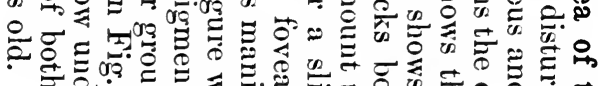
Q

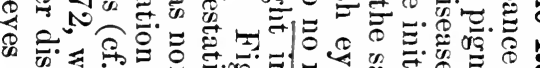

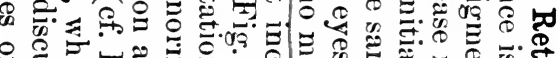

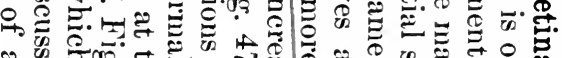

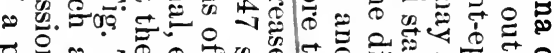

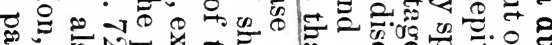

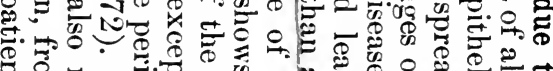

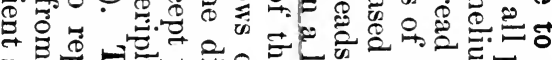

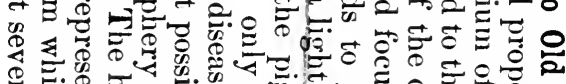

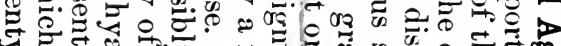

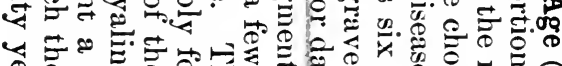

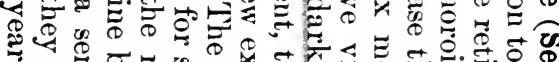

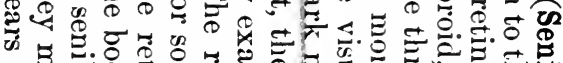

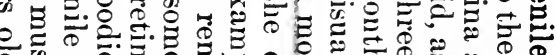

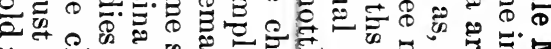

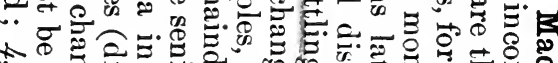
स \& क्ष

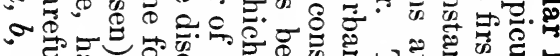

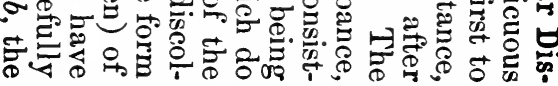




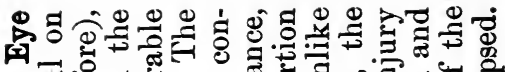

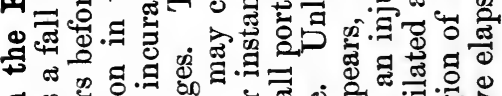
ด ข

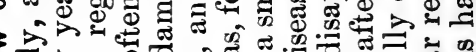

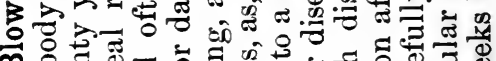
ค. ฮี ๘

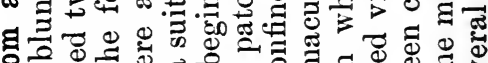
造.

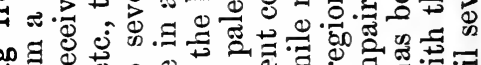

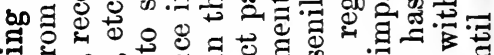

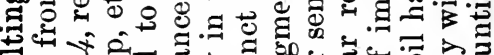

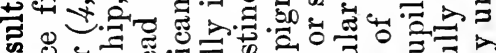

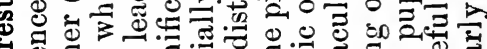

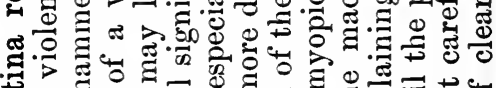
要

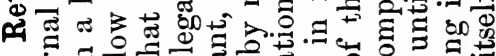
0

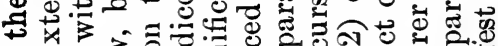

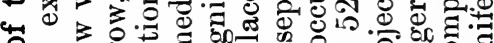
o o $\geqq .000$. ๙ के

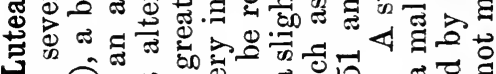
け

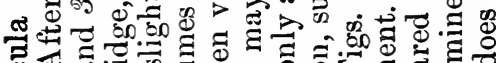

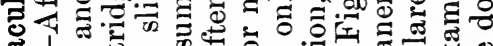

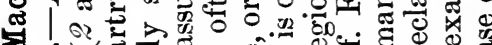

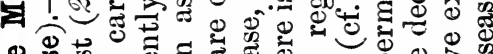

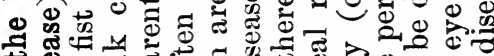

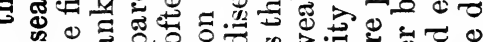

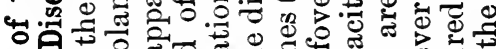

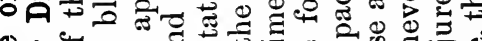

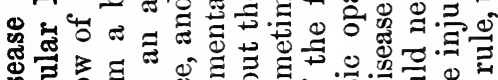
.

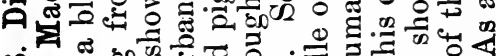
乐

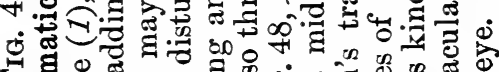
$=8$ so 0 .

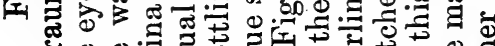

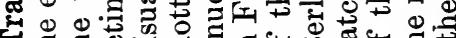

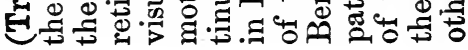


N

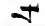
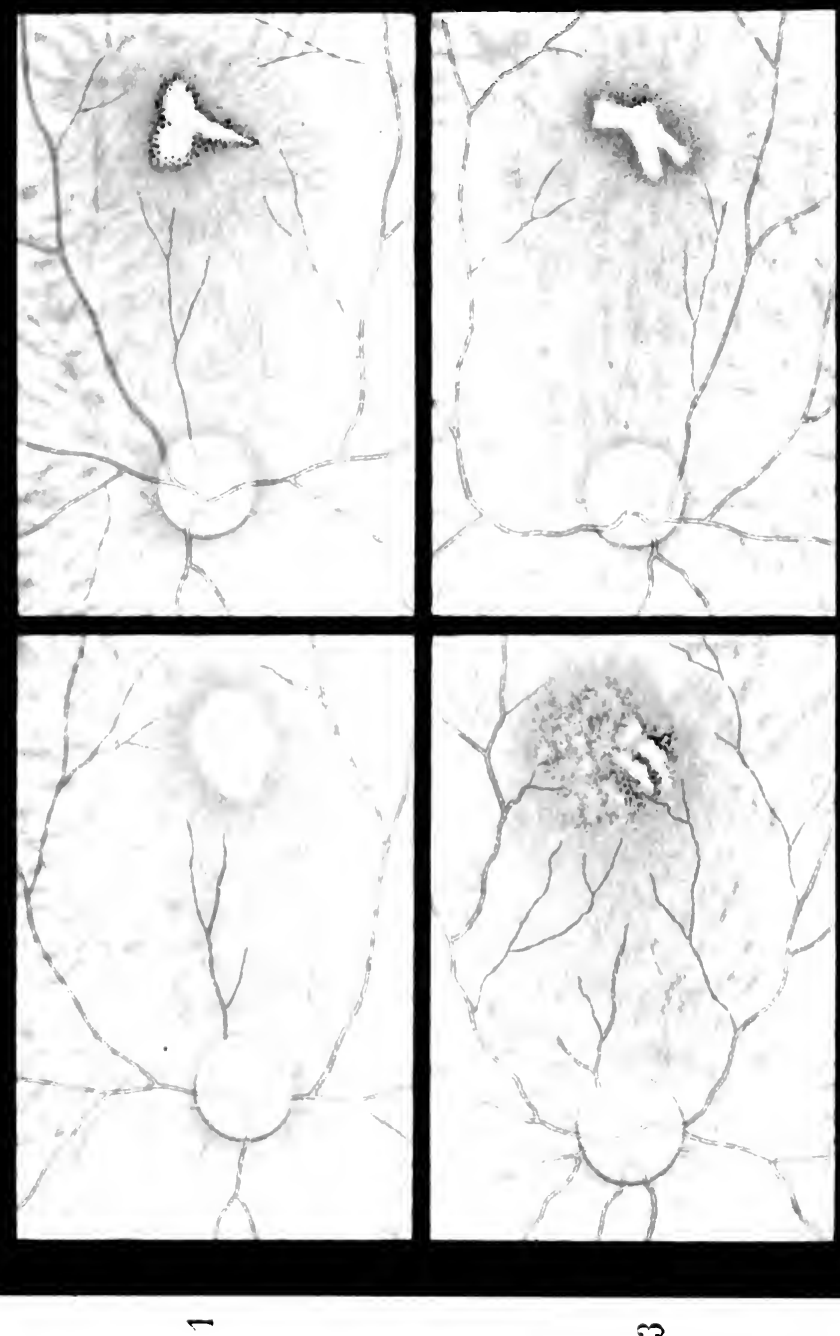


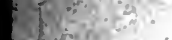

$\therefore x<x_{3}$

$=25 \times$

Whi

$\therefore$ in

$f(x)=$

ic

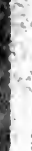

$$
\checkmark
$$

$\because$ 


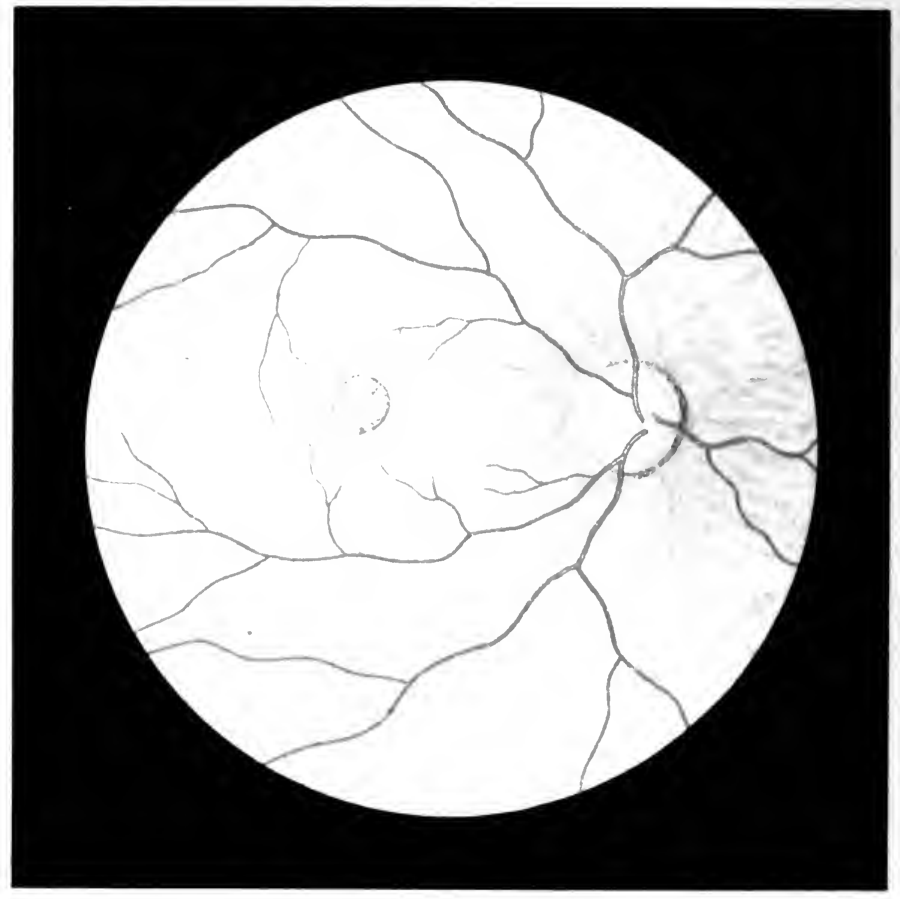

Fig. 49. 
Fig. 49. Perforation of the Macula Lutea after Contusion of the Eyeball.' The patient, a man of fifty-two, had had a stick thrown at his left eye ten years before. ${ }^{2}$ Since that time the vision of the injured eye was impaired, and at the time of my first examination, when the picture of the eye-ground was made (1891), he could only count fingers at a distance of 1.5 meters. At the center of the macula there is a circular opening with sharply defined edges about half the size of a papilla, the surrounding area is slightly opaque with minute dots and in places covered with pale glistening spots and patches. Of the latter a few are also found in the retinal defect characterized by its red color. The floor of this defect is almost uniformly red and by the direct method shows only a very faint mottling, representing the mosaic arrangement of the pigment-epithelium. But this can only be recognized with great difficulty. The rest of the eye-ground is normal.

I have frequently seen defects of this kind after severe contusion of the eyeball by a blow with a stick or fist, explosions, arrow wounds, stones, etc. The opening is (11sually circular, but may also become ovalin outline, owing to shrinking and consequent deterioration of the retina in the neighborhood of the macula. It is probable that openings of this kind in the fovea may appear spontaneously in advanced age without any traumatism, possibly owing to arteriosclerosis. In a case of this kind I saw such a perforation in both eyes in a woman sixty-four years of age, with marked arteriosclerosis and some albuminuria. (The Editor has reported similar cases and one similar lesion which appeared after non-traumatic iridocyclitis.)

1 See Haab, Die traumatische Durchlöcherung der Makula lutea. Ztschr. für Augenheilk., 1900, p. 113.

2 Case II. in the article just cited. 
Fig. 50, a. Section through the Macula Lutea and Surroundings in Macular Disease resulting from Orbital Tumor. - The specimen was taken from the eyeball shown in Fig. 54, $a$, after enucleation. The yellowish-red patch with slightly pigmented borders seen in that figure is represented in the transverse section by a separation of the pigment-epithelium in the region of the fovea centralis, where the epithelium is without pigment in places or is altogether wanting. The cones and corresponding nuclei are also wanting in considerable areas. The nerve-fiber and ganglion-cell layers also show a marked alteration. In the ganglion-cell layer the diminution of the cells and vacuole-formation at once become apparent (cf. Fig. 14, $c$, of the normal macula, which is represented under the same power). The choroid does not show any marked changes.

Magnified 30 times.

FIG. 50. $b$ and $c$. Transverse Sections through the Retina in Thrombosis of the Vena Centralis Retinæ (cf. Fig. 33, $b$ ). - Section $b$ corresponds to the neighborhood of the papilla; section $c$, to an area somewhat more remote. Hence we see in $b$ a larger and more engorged retinal vein (Ret. V.), while $c$ shows a greater number of small vessels more or less engorged by the damming back of the blood. In addition there are numerous hemorrhages of varying sizes scattered throughout the entire preparation. In $c$ many vacuoles and fissures appear in the tissue which must be referred to edema.

1 , Nerve-fiber and ganglion-cell layer; 3, inner nuclear layer ; 4, internuclear (outer reticular) layer ; 5 , outer nuclear layer; 6 , layer of rods and cones.

Sections $b$ and $c$ magnified 90 times. 


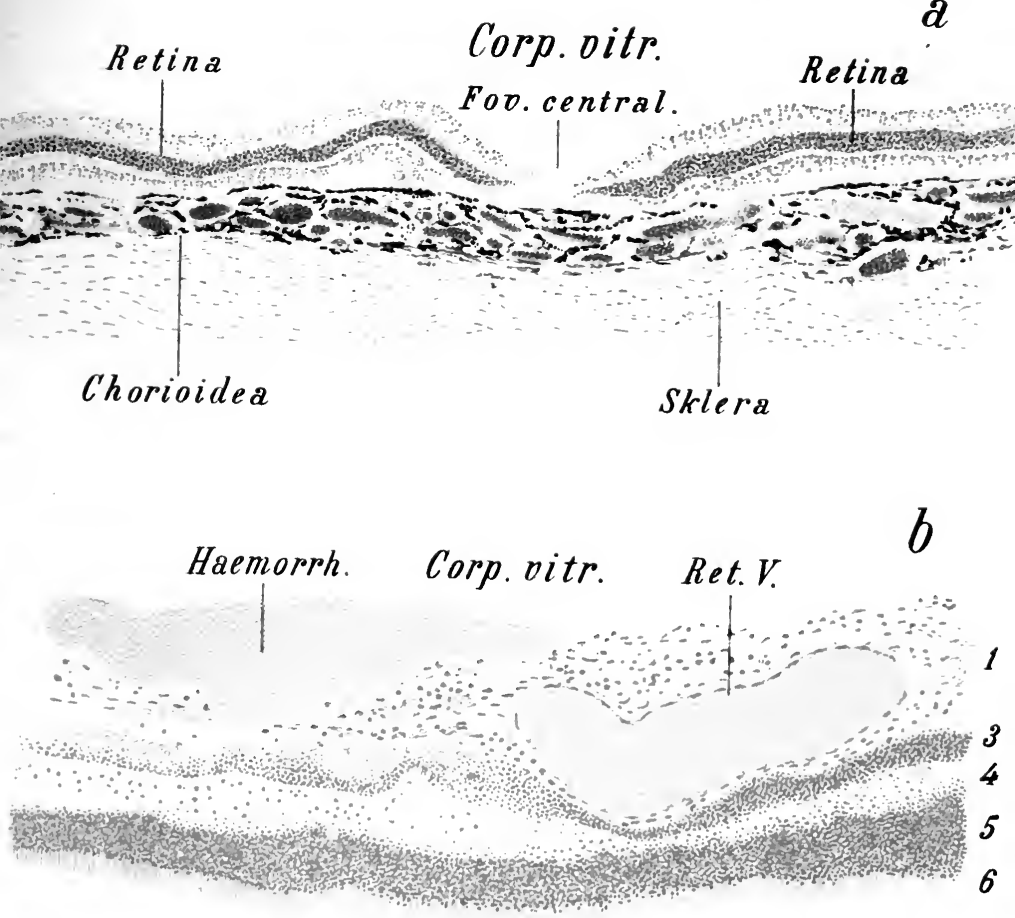

Corp. vitr.

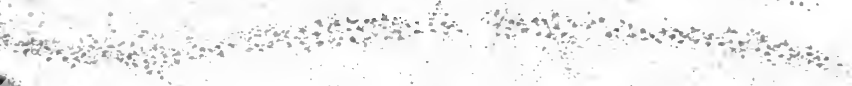

Fig. 50 . 

Fig. 51. Opacity of the Retina due to a Blow on the Eye; Berlin's 0pacity; Commotio Retinæ (direct method, reduced). - This form of opacity of the retina, which is often observed after external violence to the eye by a blunt instrument, was first described and studied by Berlin. The changes soon pass off, so that they are best seen immediately after the receipt of the injury. The opacity is often produced by a blow with a fist or stick, by striking the eye forcibly against a hard object, a blow from a stone or snowball, etc. A milkish white opacity is often seen in two separate portions of the retina, one at the place where the eye was struck and another more or less extensive area in the region of the macula lutea. The former is, as a rule, more extensive, more marked, and more persistent, while that in the macular region is less intense and disappears more rapidly. The macula and visual power remain intact. The opacity at the periphery does not, as a rule, obscure the retinal vessels. The condition has undoubt edly often been mistaken for separation of the retina. The nature of the opacity is not as yet well understood. The present picture was taken a few hours after the patient had been hit in the eye with a snowball. 
Fig. 52. Opacity of the Retina due to a Blow on the Eye; Berlin's 0pacity; Commotio Retinæ.-In this case the contusion was much more severe, the eye having been struck by a large piece of iron; the retinal opacity is, accordingly, more marked, and there are even a few small hemorrhages in the opaque area which corresponds to the site of the direct injury (the upper border of the picture, hence the lower portion of the retina). The abnormal appearances all disappeared in a few days, the opacity in the macular region first, and soon afterward that in the lower part of the retina. This traumatic opacity is readily distinguished from separation of the retina. Aside from the fact that a very recent detachment of the retina is usually more transparent instead of milky white, the course of the vessels in commotio retinx is not affected; there is no parallactic dislocation or marked degree of hypermetropia. Besides, the folds of a retinal detachment are wanting.

It is interesting to note that in these cases there is sometimes found at the center of the fovea an isolated, more or less wellmarked pale spot, distinctly separated from the remainder of the macular opacity. This is probably not caused by opacity of the retina because the membrane is exceedingly thin at this point; it is probable that its substratum is situated behind the floor of the central fovea, perhaps in the pigmentary epithelium, perhaps between the latter and the layer of rods and cones. The phenomena is also transitory. 


$$
0
$$





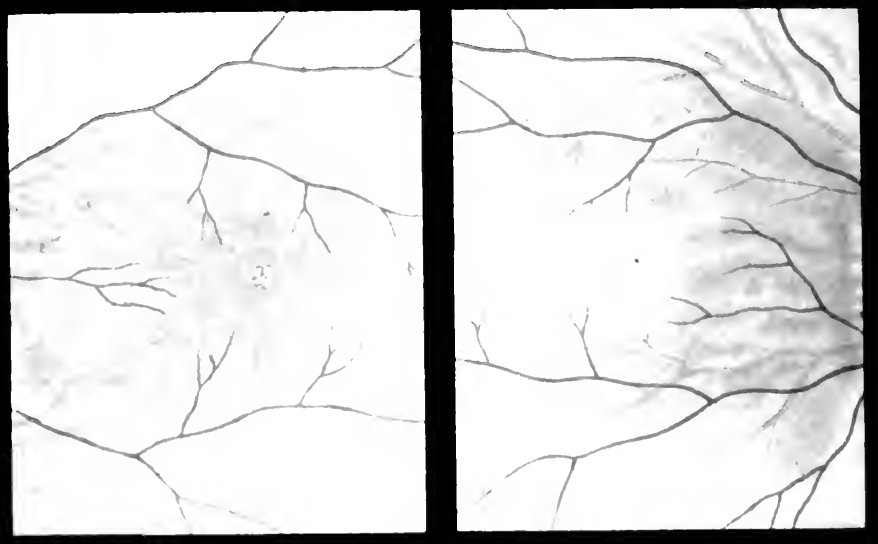

b
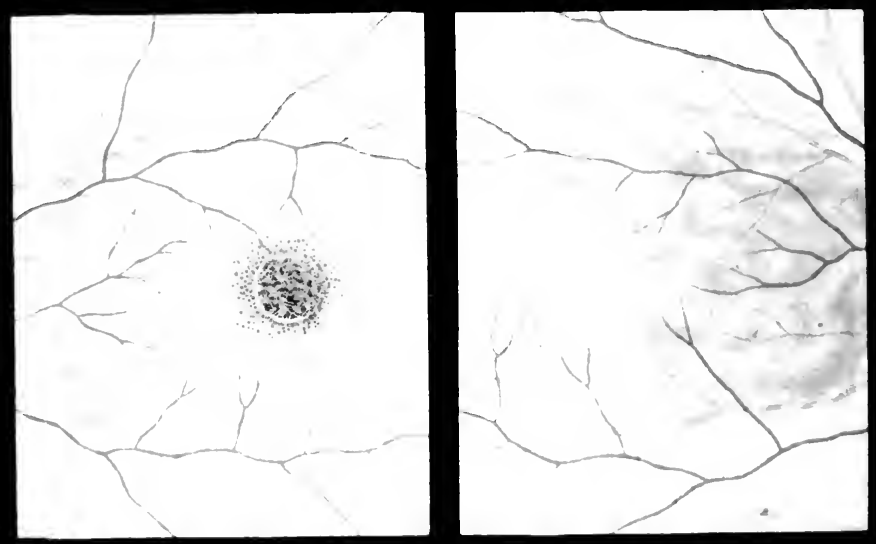

d

Fig. 53. 
Fig. 53. Disease of the Macula Lutea due to the Presence of a Foreign Body in the Vitreous.-Such is the sensitiveness of the macula lutea that even aseptic bodies that have penetrated into the deeper layers of the eye often produce, after a short time, an isolated disease of this portion of the retina without directly injuring the macula. Fig. 53 shows four examples of this kind: The small grayish white spots at the center of the macula seen in $a$ were produced by a particle of a copper shell, which remained five days in the vitreous body. The spots disappeared completely after the splinter was removed, and two and a half months later vision was entirely restored.

$b$ shows a yellow discoloration of the macular region a year after a particle of copper (from a cap) entered the anterior portion of the eye. The foreign body was not removed and produced the changes shown in Fig. 74 , which represents a later stage. Eventually the discoloration decreased, and at the present time of writing, seventeen years after the injury was sustained, vision is still $\frac{1}{3}$. This, however, is an exceptional case.

The round grayish spot shown in $c$ was produced by a steel splinter that remained in the retina twenty hours. The splinter which is shown in Fig. 55, $a$, was drawn back into the anterior portion of the eye by means of a large magnet and thus removed. The center of the macula at first showed a yellowish pigmentation which was gradually replaced by the dark spot seen in the picture, painted three months after the injury was sustained. As a consequence, vision was reduced to $\frac{1}{10}$, the condition of the eye in other respects not being impaired.

The delicate pigmentation shown in $d$ is owing to the presence of an iron splinter from a hook in the vitreous, which remained a week and was then removed by means of a small magnet. Vision was reduced to $\frac{1}{7}$. 


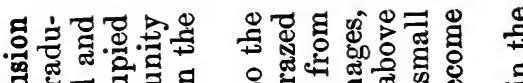
(1)

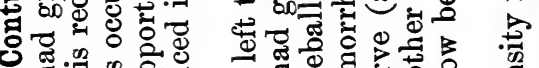
0.

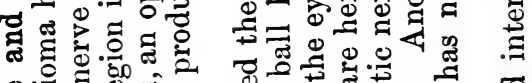
o.

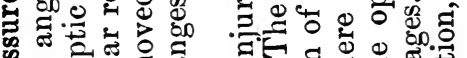
要

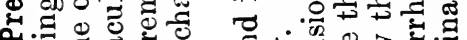

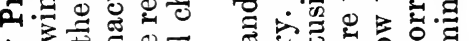
응 (5)

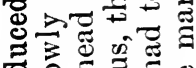
का का 出

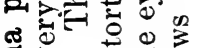
政 क्षे कि

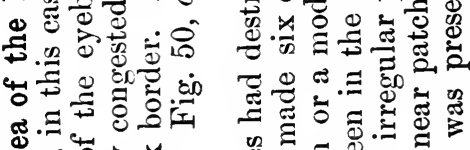

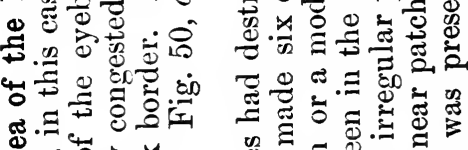

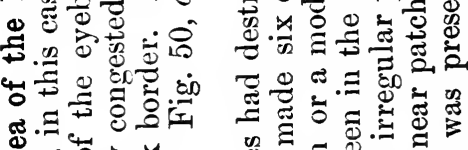

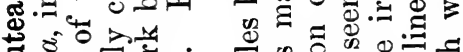

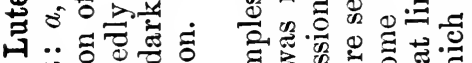
$+\frac{0}{0} 0.0$

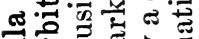

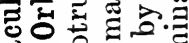
$\rightarrow$ a :

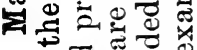
0 的 5.

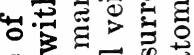
o ⿷ o.

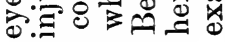

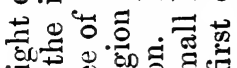
ज.

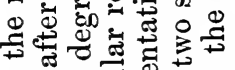
on 0 石

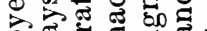

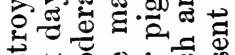

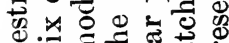

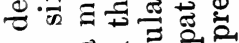

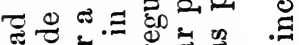
Fi $\stackrel{\infty}{\Delta}$ ฮี की $+2 \dot{1}$

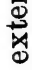
용 ヨ व 궁 ठำ ज्ञ ठิ : య

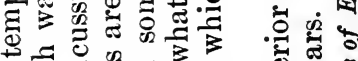

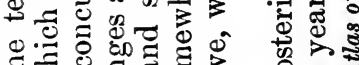
光

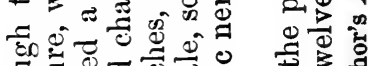
ప

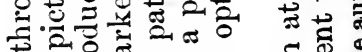

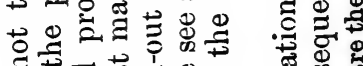

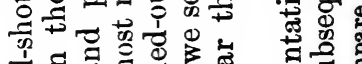

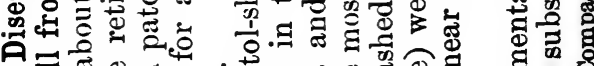

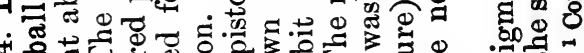
में ஸी $\overbrace{0}^{0}$ : $\because 4$ \&

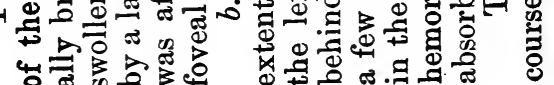




$$
8
$$





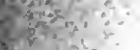

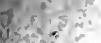

$4=2$

$q^{2}=2$

in

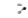

$\bullet$

$x_{i}$

it

.

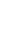




$$
8
$$




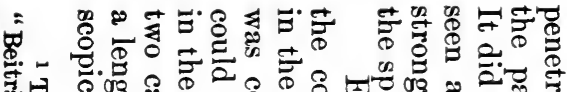
氙点 व

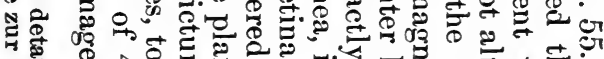

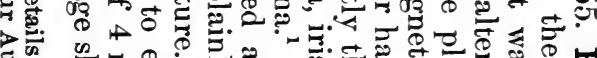

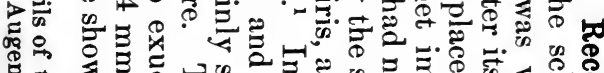

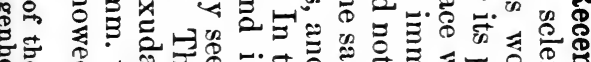

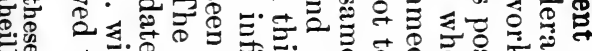

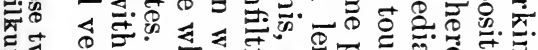
荡

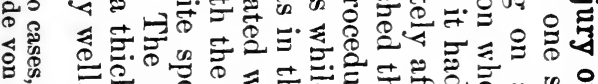

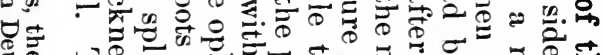
若

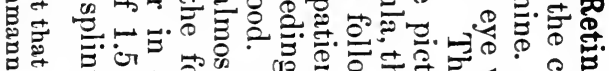

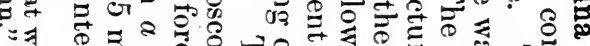
串

w象

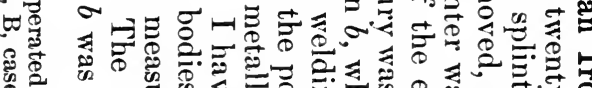
○ ᄋ유

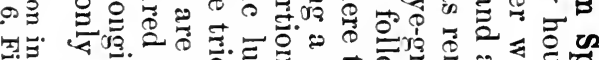

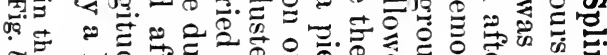

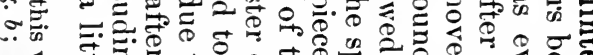
赵 की

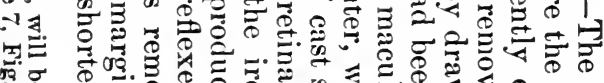

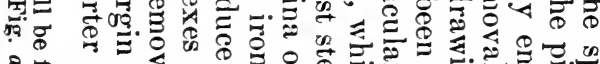

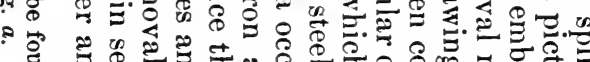

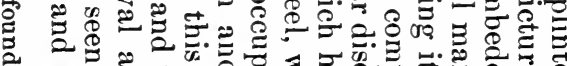

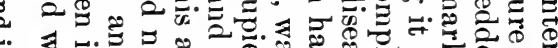
F. 田 द 舟

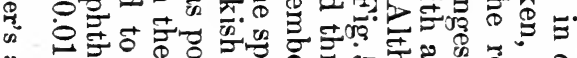

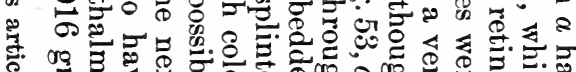

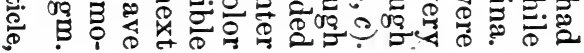


Fig. 56. Air-bubble in the Upper Portion of the Vitreous due to the entrance of an iron splinter (direct method, reduced).-The accident occurred shortly before the picture was made, while the patient was working with iron, and the splinter became embedded in the lower part of the retina. Its entrance was followed by the formation of an air-bubble which disappeared after a few hours. These air-bubbles always disappear very rapidly, so that they can only be seen immediately after the injury. Several air-bubbles of this kind may be found in the vitreous body after the entrance of a splinter. Hence air in the vitreous is an important diagnostic point, indicating a foreign body in the eyeball. Occasionally, although rarely, the mere injury of the eye by a splinter may, without its remaining in the eye, be followed by the entrance of air into the vitreous, so that the finding of such an air-bubble does not positively indicate the presence of a splinter in the eye.

Air-bubbles in the vitreous look like air-bubbles in a microscopic section.

In this case the splinter was immediately removed by means of the author's large magnet, whereupon the wound rapidly healed and vision was completely restored.

For further details about my method of operating with the magnet, the reader is referred to Haab's Atlas and Epitome of Operative Ophthalmology. 


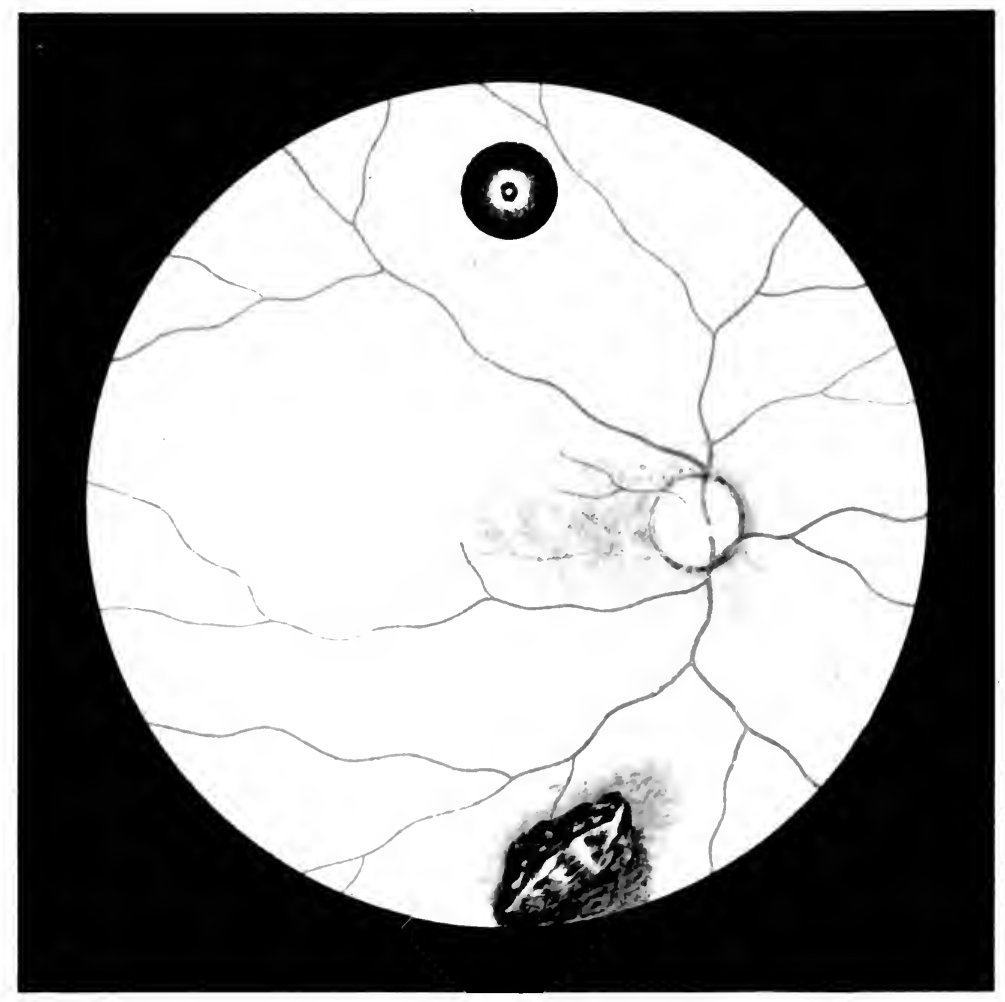

Fig. 56. 



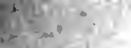

$3 y^{2}=$

is

$\vdots$

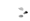

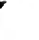

$$
-
$$$$
b^{\prime}
$$

$$
\text { . }
$$

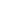

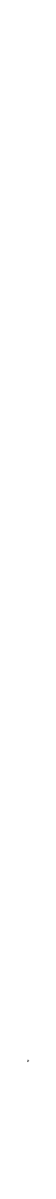

tast 


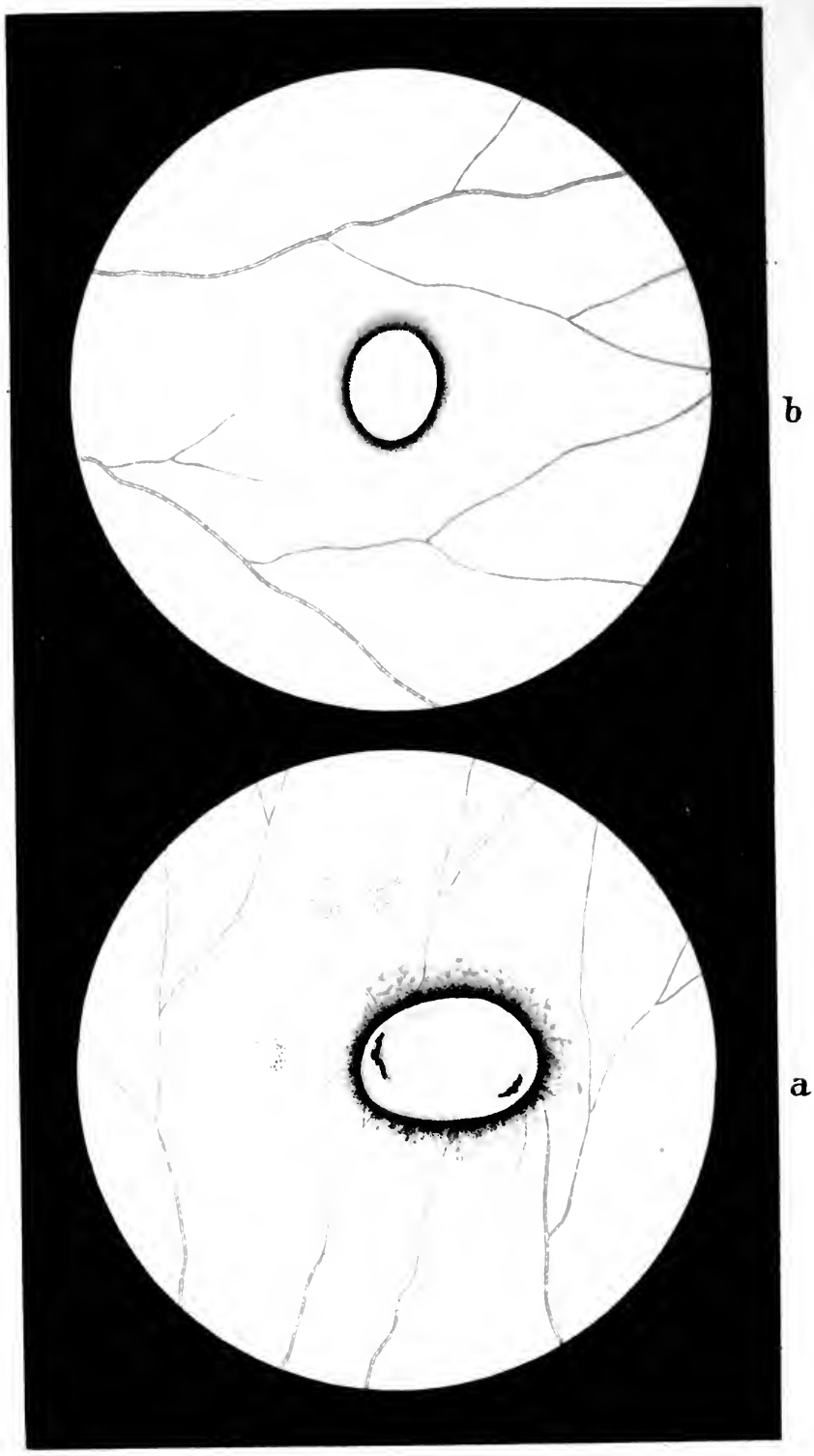

Fig. 57. 
FIG. 57. Old Injury of the Retina by an Iron Splinter-In the two cases shown in the foregoing figure, the foreign bodies had been only a short time in the retina and either lay apparent on the surface or were embedded within the membrane; the present figure shows two cases in which the splinters had penetrated the eye some time before and now present a somewhat different picture. The foreign bodies are here covered by a whitish exudate, the black color of the metal showing only in two places in $a$. In the latter the splinter, which had been almost two months in the retina, produced a characteristic alteration in the immediate neighborhood. The pigment-epithelium has in part disappeared and the splinter is surrounded by a light colored areola in which a few choroidal vessels are seen. This areola also shows an irregular pigmentation, which becomes more intense near the foreign body. In the adjacent region there are three small choroiditic foci, light in color, with ill-defined edges, and surrounded by irregular pigmentation.

In $b$ there are no changes in the immediate surrounding of the splinter, although at the time the picture was made the splinter had been in the retina six weeks. (For further details concerning this case, see Hürzeler, loc. cit., B, case 4.) 


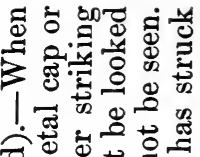 \\ co}

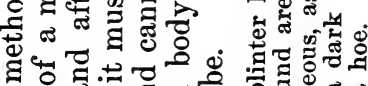

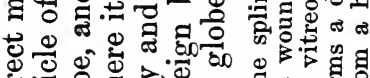

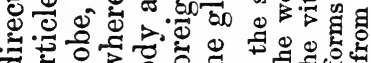

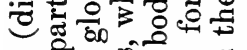

等

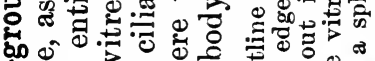

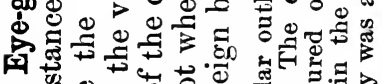
尊. 政

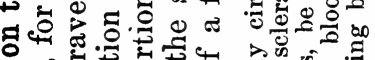

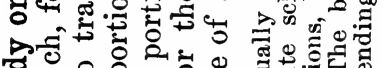

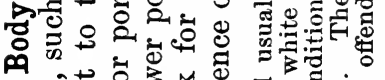

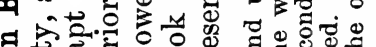

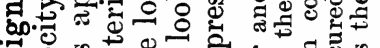

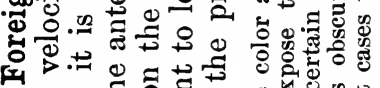
का tै 0 to. 政

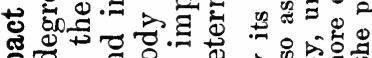
等 $7 x_{0}$ 1. 落 경종

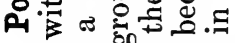
$\dot{0} \Rightarrow$ d

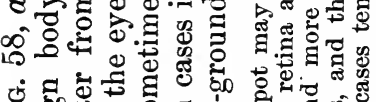
o.

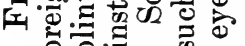
कै 0

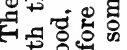

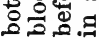

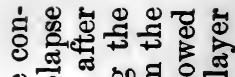

के

20:

诂专

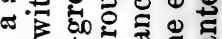

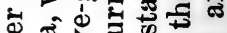

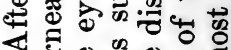

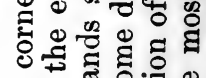
它包 西 政

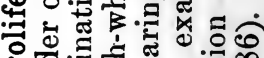
要.

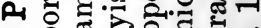
on 땡혀 言宊 $00.0 \%$

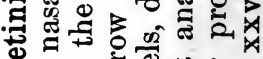

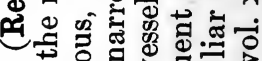

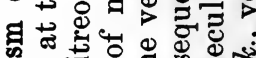

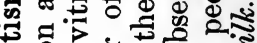

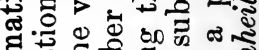
हुำ 政

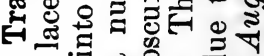

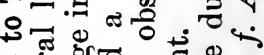

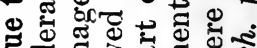

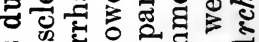

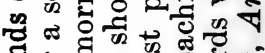
สี so $^{\circ}$. ค.ศี.

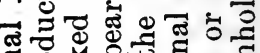
. 은 은

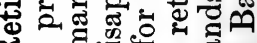
ผ

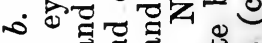
$\infty$ డె డే

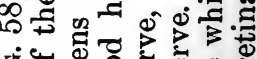

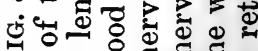
我 0 융 .

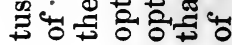




$$
8
$$



$\therefore \therefore v^{\circ}$

$\because \therefore,-\infty$

$\therefore$.

:

$1=$

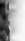

3

" 


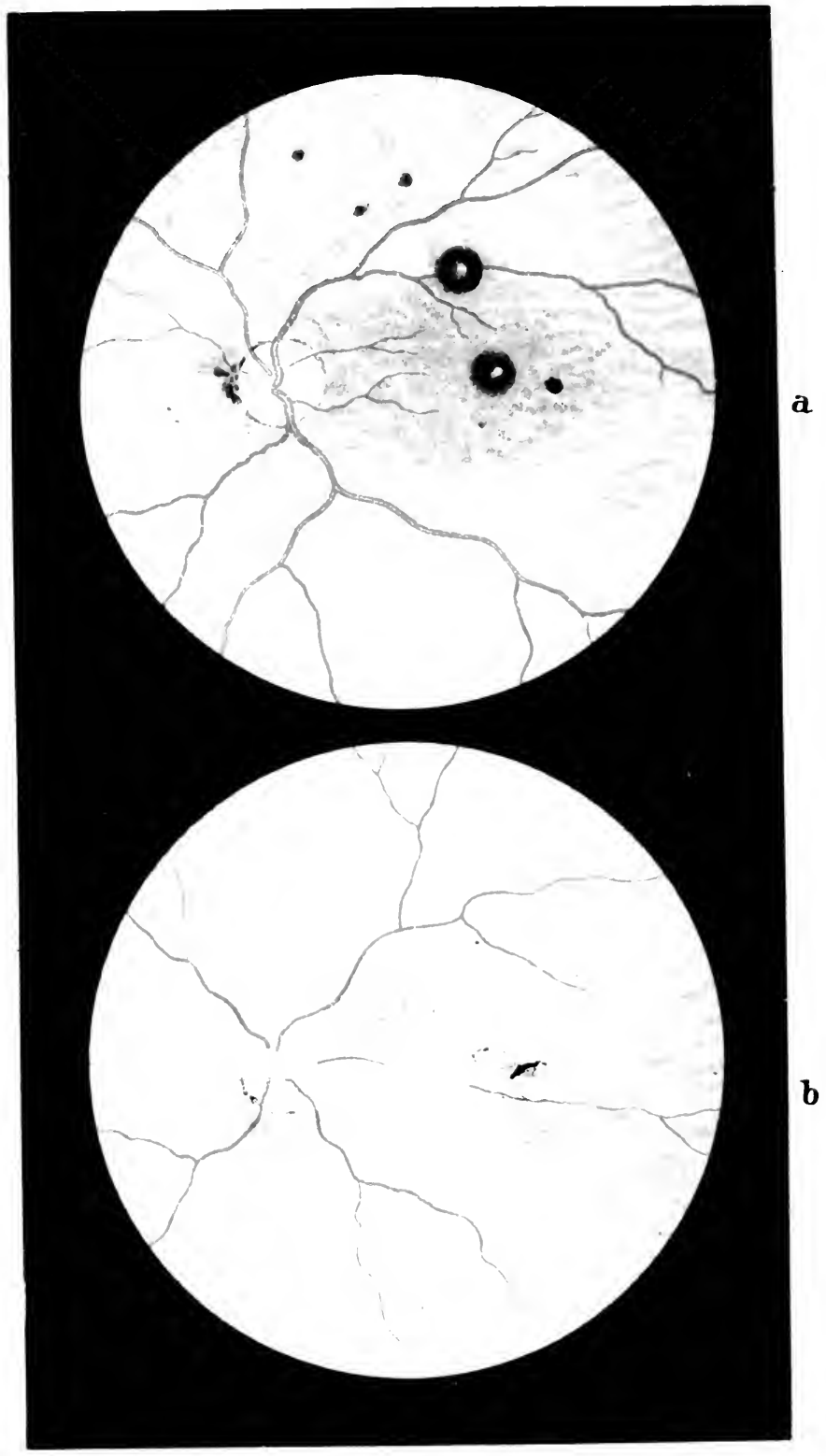

Fig. 58.I. 
ब

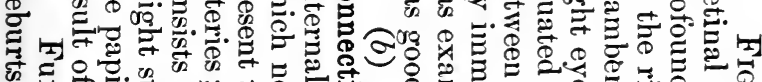

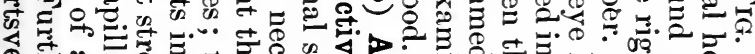

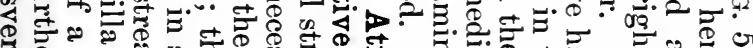

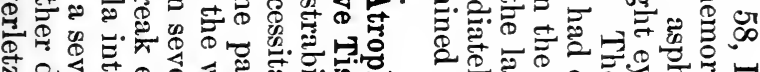
융

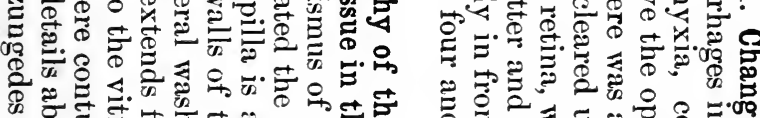

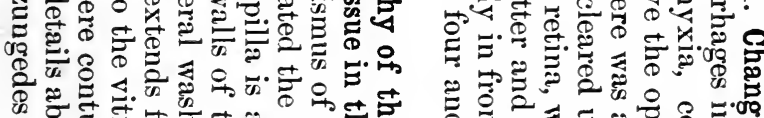

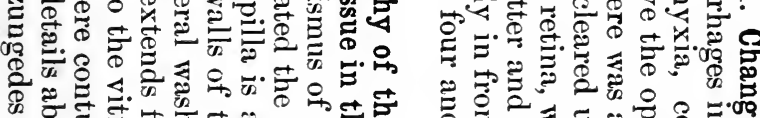

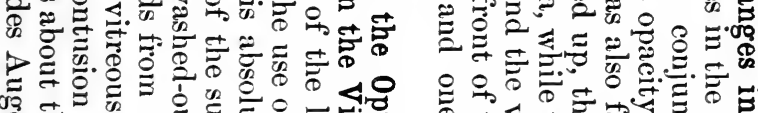

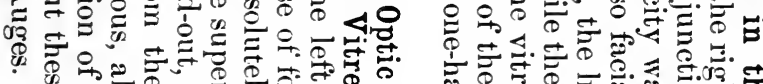

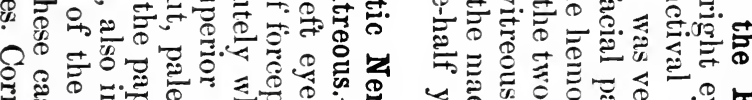

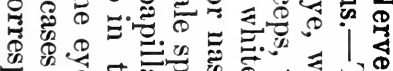

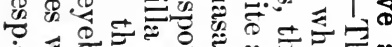

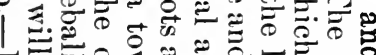

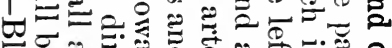

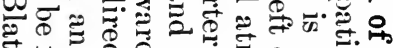
त $520+5.500$

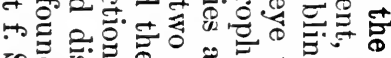

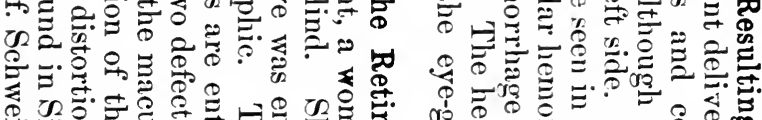

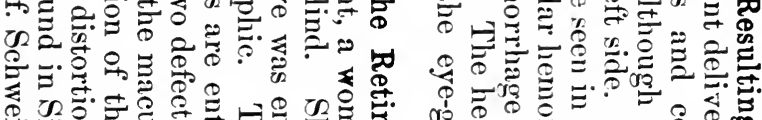
通.

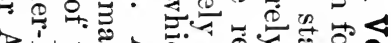

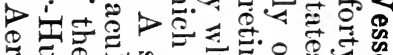

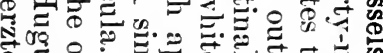

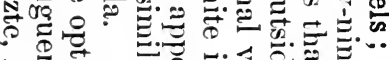

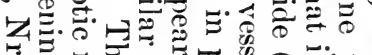
$\because$ :

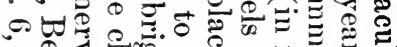

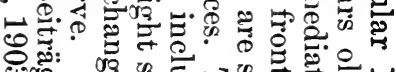

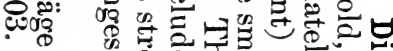
N

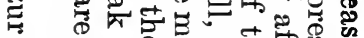

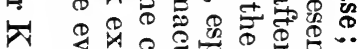

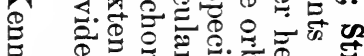

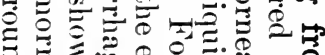

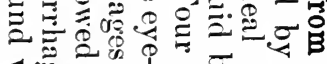

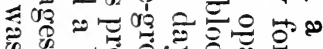

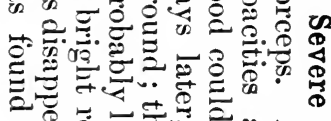

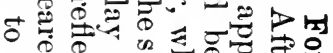
- 乞 ڤ

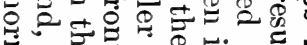

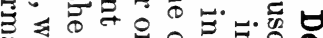

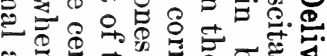

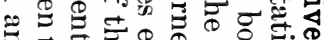

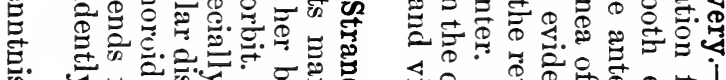

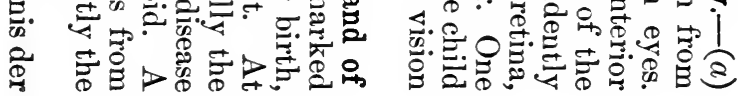


Fig. 59. Retinal Detachment in the Temporal Portion of the Eye-ground.-Aithough the injury is one of about twelve weeks' standing, it may be called a recent injury, as there is not as yet any marked degree of opacity or wrinkling of the retina. 'The picture of the retinal detachment shows one important phenomenon, namely, the absence of the choroidal striations in the detached-area, the striations being obscured by the slight opacity of the retina, as also by the subretinal fluid. In the marginal zone of the macula, where the retina is thickest, the opacity is most marked, so that the central depression, which is the thinnest portion, appears more bright red by contrast; on the other hand, the red color of the choroid shines through more distinctly than in other kinds of macular opacities, because the retina is thin in the region of the fovea on account of the atrophy of its elements. Occasionally, after the retinal detachment has lasted for some time, an actual perforation may even be observed in the center of the fovea.

The detachment, which in this case occupies the temporal quadrant of the eye-ground, is quite superficial-that is to say, not very far removed from the choroid. In the macular region there is an hypermetropia of $4.0 \mathrm{D}$; toward the periphery the hypermetropia is 7 to $8.0 \mathrm{D}$, while at the papilla the eye is emmetropic. Vision is reduced to power of counting the fingers at a distance of 3 meters. 
<smiles>C=[IH]</smiles> 


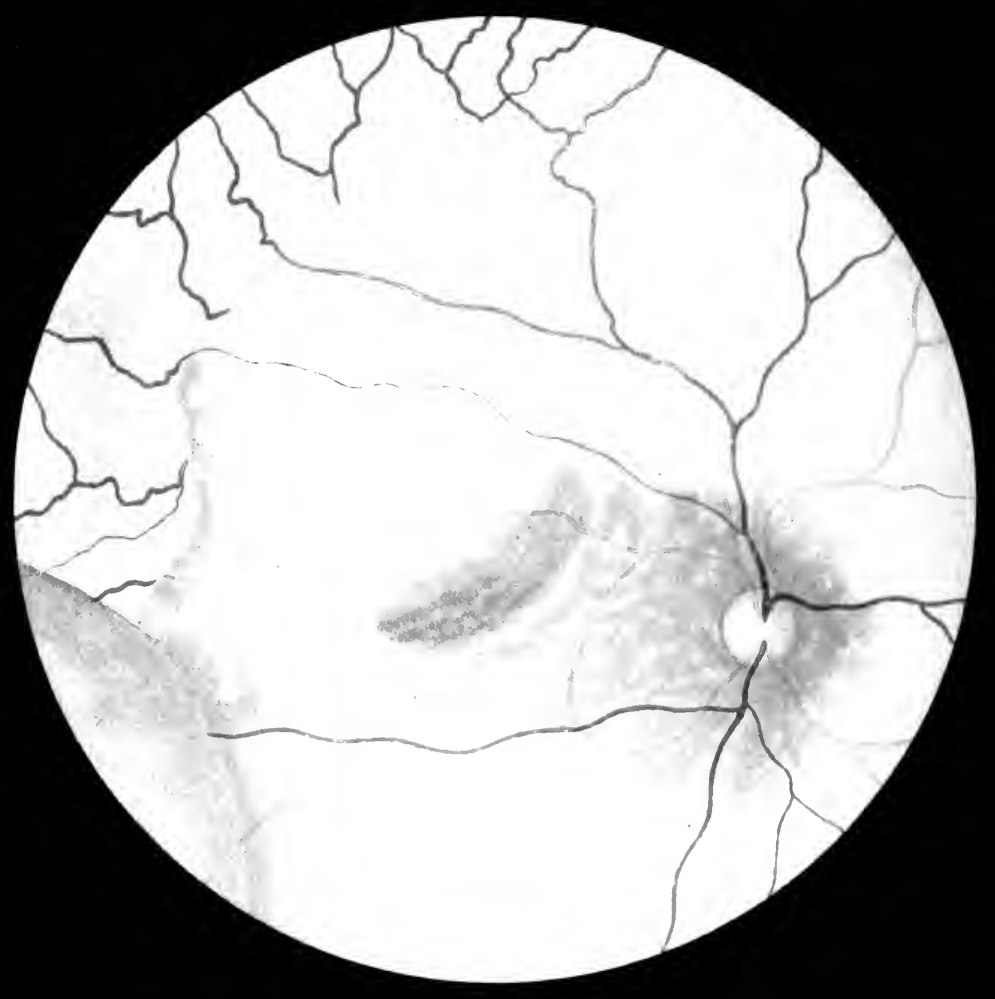

Fig. 60 . 
Fig. 60. Retinal Bands and Retinal Detachment after a Punctured Wound of the Eye.-Four years after an insignificant injury of the eye from a sharp piece of tin, which had pierced the sclera to the temporal side of the cornea, vision began to deteriorate. As the picture shows, there was a grayish-white exudate, spherical in shape, in the outer and upper portion between the corneal border and the equator, projecting some distance into the vitreous, seen in the picture at the left lower border. Below this exudate, which occupied the site of the puncture (and touching it above in the picture), the retina was detached, and this detachment extends some distance along the upper border. Wherever the retina is detached the color is paler, and we see folds and irregular tortuous vessels somewhat darker in color than the other vessels in the picture. Between the detachment (in the lower portion of the eye) and the exudate are a number of white bands or lines, some of which anastomose, although in the main they run in the same general direction. In the portion occupied by these lines there is no retinal detachment. I am unable to determine whether these bands are of the same character as those shown in Fig. 58, b. For weeks they showed no change. The macular region presented abnormally heavy, somewhat irregular pigmentation. The retinal detachment is likely to increase as time goes on. 
Fig. 61. Retinal Detachment at the Inner Upper and Inner Lower Portion of the Right Eye (indirect method).At the inner upper portion there is a slight tear in the detachment through which the red color of the choroid is seen. Toward the nasal side there is a strip of retina that has not yet become detached. Above and below this strip the detachment is quite marked (the detachment became even greater later on, in spite of treatment).

In this case the detachment which had existed for three months appeared quite suddenly. Both eyes had been operated on thirty-five years before for lamellar or congenital cataract; in the left eye the pupil was cloudy, but the right eye had fairly good vision up to the present time in spite of the aphakia. From the presence of a fine band in the anterior portion of the vitreous, which otherwise is quite clear, it may be suspected that an injury of the vitreous with prolapse occurred at the time of the operation.

This case shows excellently the great danger of injury of the vitreous, and that the retinal detachment which it produces may, under certain circumstances, be greatly delayed -in this case thirty-five years. 



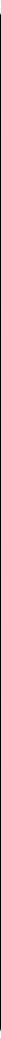

Fig. 62. 
Fig. 62. Retinal Detachment (Solutio Retinæ) with Laceration.-It is not uncommon to find in retinal detachments a tear or hole of varying size and shape in the detached portion of the retina. The opening is quite often surrounded by a shred of the membrane of a corresponding outline, which appears to have been torn out, and then reflected or puckered. In the present case the tongue-shaped portion of retina extending from the lower margin of the picture toward the red, approximately quadrangular opening, in the detached portion of which it forms the lower border, was probably torn out of the detachment, and thus produced the opening. According to Leber, the vitreous as it shrinks strips the retina from the pigment-epithelium and occasionally produces an opening corresponding to a spot where it is more firmly attached to the retina. Through the opening, the edges of which are somewhat reflected, we see the choroid with its vascular striation, which is very pronounced above, where the retina is normal and transparent. Toward the left in the picture the detachment is beginning to spread. At this point it is quite superficial. The optic nerve is invisible, being behind the retinal detachment. 
Fig. 63. Hemorrhagic Retinitis of Pregnancy.-In spite of the presence of the stellate figure in the macular region, no albumin was found in repeated examinations of the urine. After the woman's delivery at term, the disease, which affected the left eye, subsided spontaneously within three weeks. The entire mass of hemorrhages and white degenerated patches seen in the picture disappeared completely, although the woman was extremely anemic both before and after her delivery. Vision also was completely restored. The right eye was not affected. It may be that we have to deal with an incomplete thrombosis of the central vein, which only partially occluded the lumen of the vessel. 


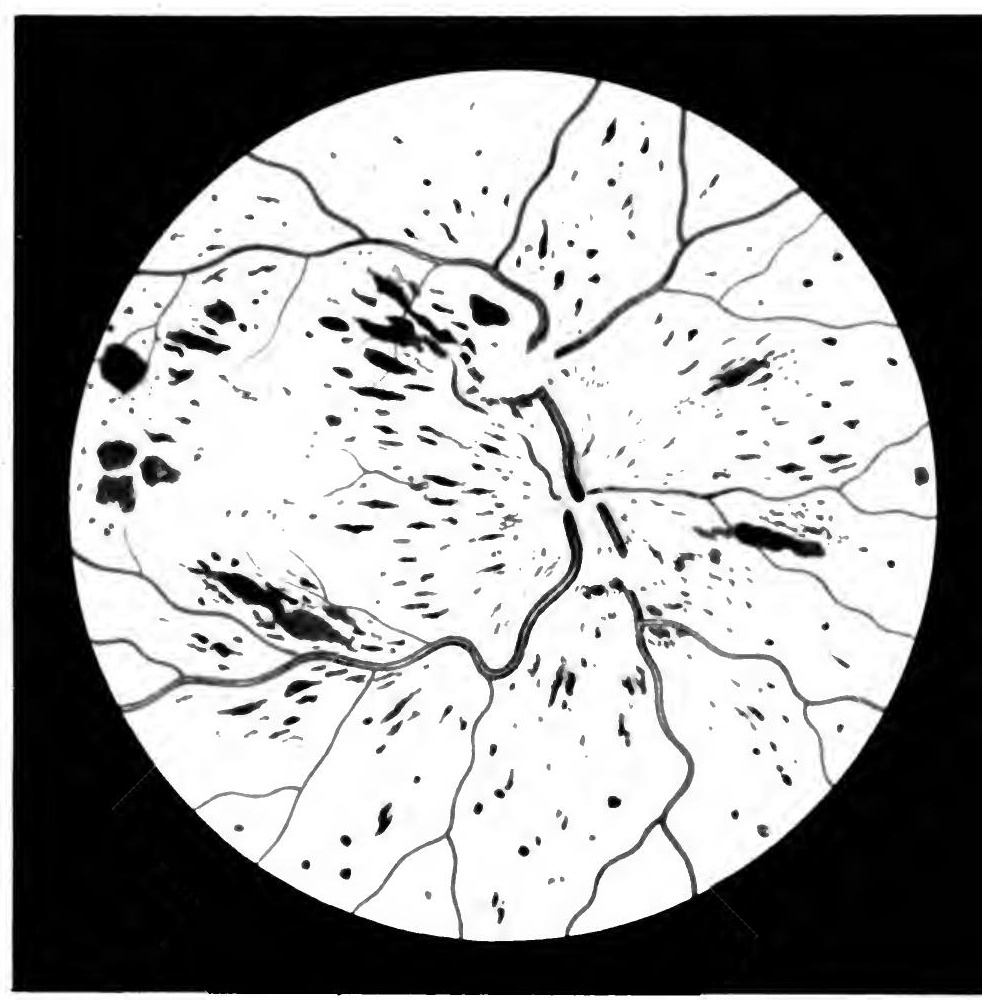

Fig. 63. 

FIG. 64. Retinitis Circinata (recently described and named by Fuchs), seen in the right eye of an otherwise healthy man seventy-seven years of age, whose other eye was normal.A number of isolated and coalescent, glistening white patches; closely resembling in color those seen in albuminuric retinitis and diabetes, are grouped about the macula in the form of an oval. At the broadest portion of this girdle surrounding the macula are a number of isolated whitish patches and dots, some of which resemble crystals. Here and there within or among the white patches are small round hemorrhages. The white patches are traversed by the retinal vessels, which usually do not show any changes in the ophthalmoscopic image in this disease.

The macular region is slightly opaque and the foveal reflex is absent. As vision is only one-fiftieth, it may be assumed that marked signs of macular disease would otherwise appear in the microscopic image. A little to one side of the center of the fovea are a few pale patches and one dark spot which occupies the pigment-epithelium. In other cases marked changes were observed in the macular region, consisting of yellowish-gray or yellow, irregular, washed-out patches.

The rest of the eye-ground is normal. At the temporal border of the papilla there is a narrow sickle, although there is no myopia. 
Fig. 65. Changes in the Eye-ground in Leukemia.-The case is one of splenomedullary leukemia with enormous splenic enlargement in a young patient of Prof. Eichhorst. The disease began about a year and a half ago. The patient does not as yet complain of any visual impairment. Although the number of leukocytes is greatly augmented, the eye-ground is not paler than normal; in fact, there are at the periphery a number of darker patches due to increased pigmentation. The most conspicuous feature is that the arteries and veins of the retina have the same coloring, the veins being only recognized by their tortuosity, which is excessive, and their increased diameter. The color of the retinal vessels is almost white, this being due in part, no doubt, to the pale color of the blood, but more particularly to changes in the vessel-walls, especially at the periphery, where we observe minute light and dark dots and a few larger pale patches; also two hemorrhages with pale centers. Round the optic nerve and the macula the retina is slightly opaque. The papilla is cloudy and paler than normal and its outlines are obscured. The changes are about equally marked in both eyes. 
FIG. 66. Glioma of the Retina.-The picture was taken from a boy two and a half years of age, who came to me with advanced gliomatous proliferation in the left eye. A careful examination of the right eye revealed in the nasal portion of the eye-ground a small glioma, appearing as an oval grayish nodule with a rounded though somewhat irregular surface. The growth was sharply outlined and the surrounding tissues showed no changes. 'The rest of the eyeground was quite normal, the pigmentation is not marked, and the choroidal vessels are plainly seen. Although this nodule grew very slowly, the neoplasm in the other eye probably spread to the brain, so that death ensued at the age of three and a half years. 
FIg. 67, $a$, explains the ophthalmoscopic picture of the Retina in Pernicious Anemia portrayed in Fig. 31.-The figure shows a small portion of the retina in transverse section containing the hemorrhages colored a bright red with eosin, especially in the anterior layers of the retina. They are especially plentiful round the blood-vessel $V$, where there is also a slight hemorrhage in the internuclear (outer reticular) layer.

Magnified 90 times.

Fig. $67, b$. Small Inflammatory Focus in Disseminated Choroiditis (superficial view).-The choroidal vessels seen in Fig. 70 do not appear in this picture. On the other hand, owing to the high power, the variegated form of the pigmentcells of the choroid and the nuclei of the cells which compose the infiltrate can be made out. The picture was taken from a thin longitudinal section of the choroid.

Magnified 112 times. 


\section{Corpus vitreum}

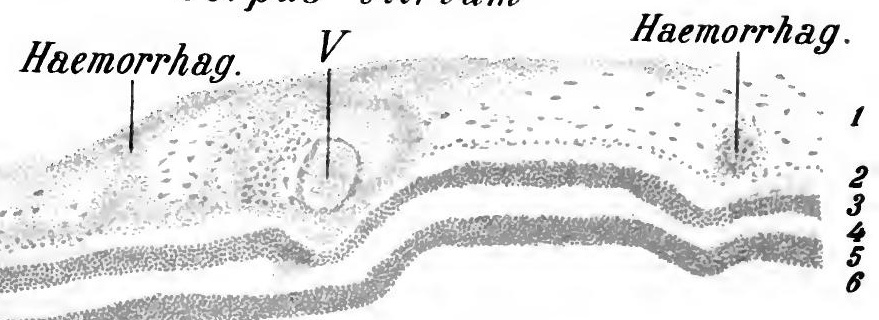

\section{Chorioidea}

\section{$b$}

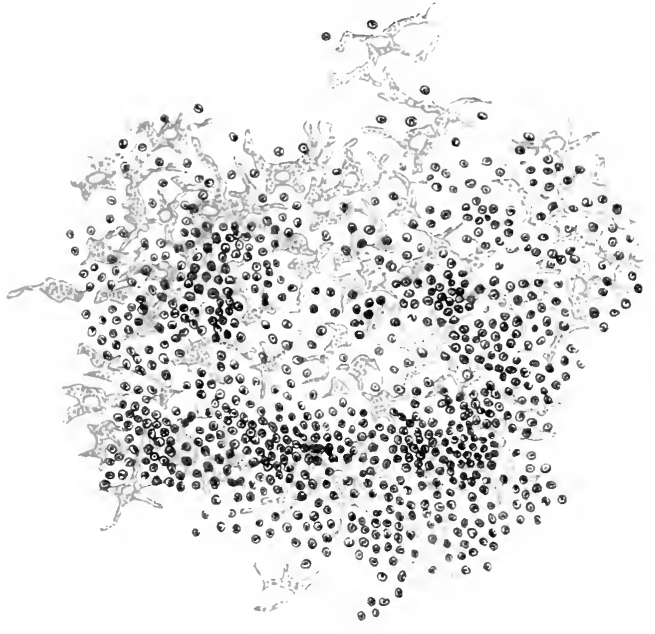

Fig. 67. 

$\therefore$ 


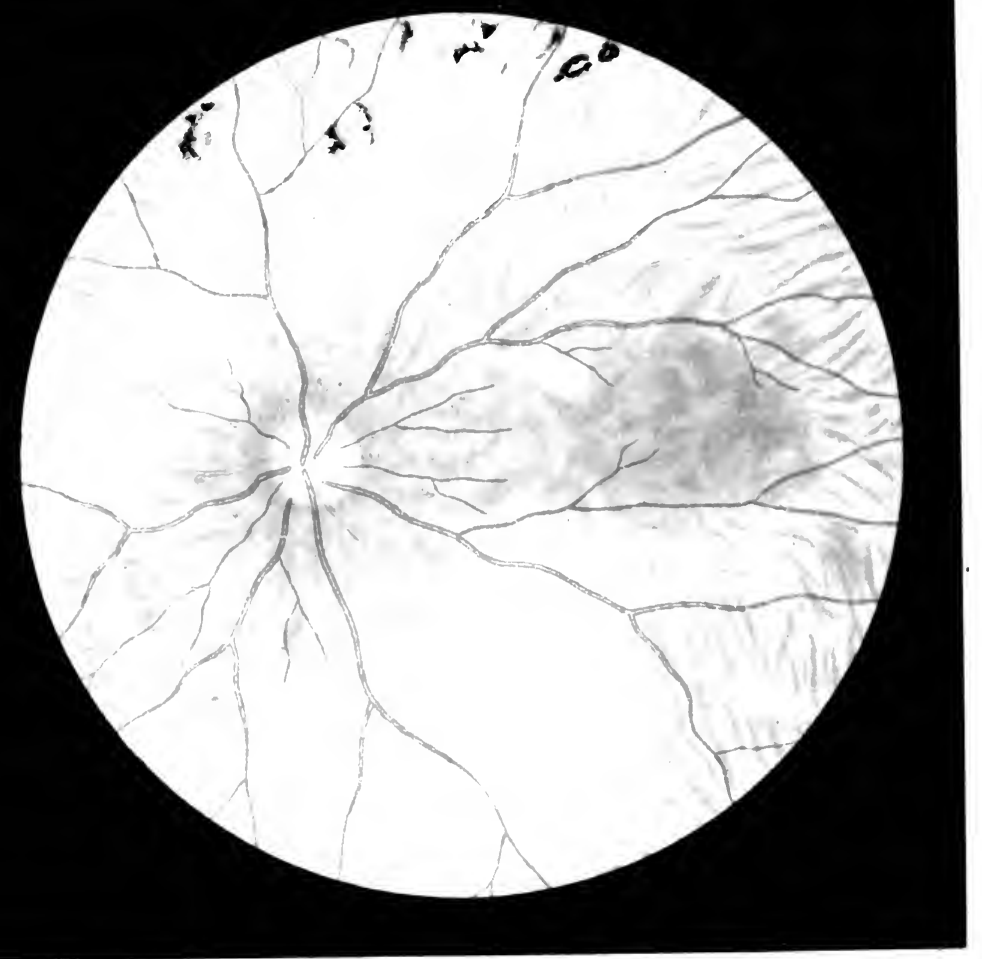

Fig. 68. 


\section{DISEASES OF THE CHOROID.}

(Figs. 68-86.)

FIG. 68. Recent Disseminated Choroiditis. - Inflammatory foci in the choroid are recognized in the main by the fact that they are traversed by the retinal vessels. In the early stages they appear either yellowish-white or grayish-white with ill-defined outlines. The condition is soon complicated by changes in the pigment and the margins of the foci become dark, or else the center appears more deeply colored. In addition to the pale fuci there may be a greater or lesser number of darkly pigmented patches which may have any shape. In the case before us we have to deal with the common variety where the foci in the choroid are circular in outline. These patches may coalesce and thus form oblong and irregular patches. With the exception of a few at the upper border of the figure, which already show a dark border, all the foci in this case are recent, as we see by the haziness of the outlines and the yellowish-gray or yellowish-red color. The nasal half of the optic nerve is somewhat reddened and the veins of the retina are slightly more engorged than usual. Foci of this kind, as a rule, tend to become converted into white patches, owing to atrophy of the choroid, which allows the sclera to shine through. Proliferation of the pigment, as a rule, is superadded, as we see in the upper portion of the picture and especially in the next figure.

The inflammation is due to accumulations of round cells, the surface view of which is shown in Figs, 67, b, and 70, and a transverse view in Fig. 79, $a$. The latter figure also shows, in addition, the subsequent anatomic changes that occur in this form of inflammation. 
.2

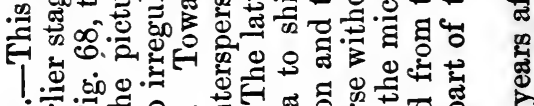

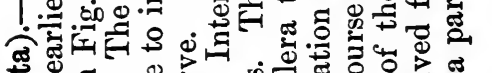

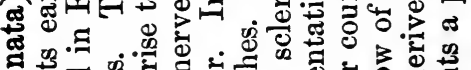

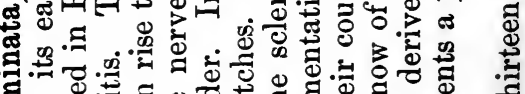

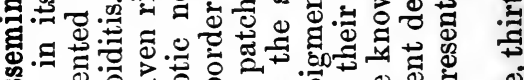

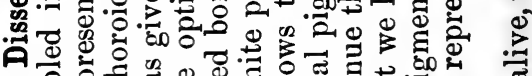

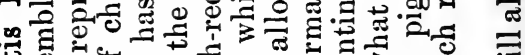

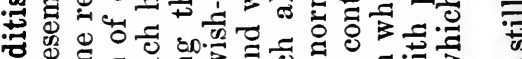

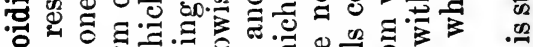

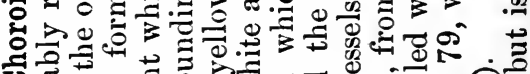
已. o 0 光

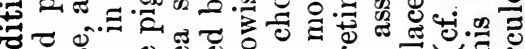
讨

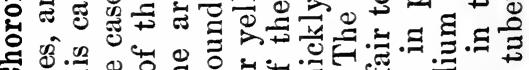

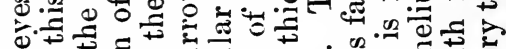

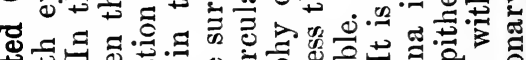
要 을 嵫. 势 A 造 4.

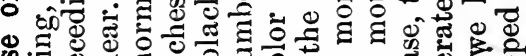

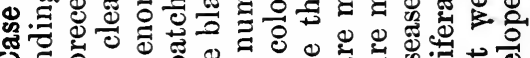

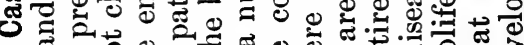

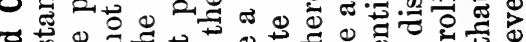
o 0 in

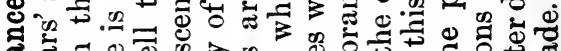

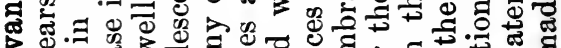
b.

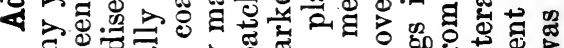

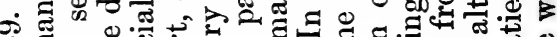
8 政 0

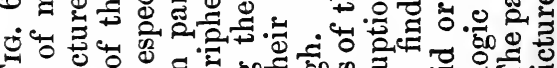

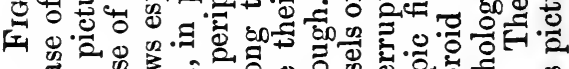

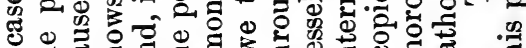

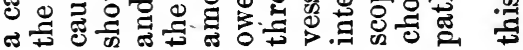




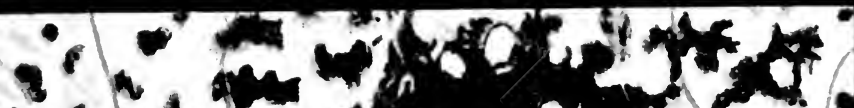

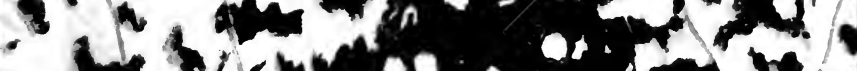

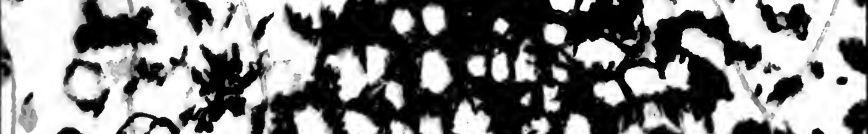
DQP

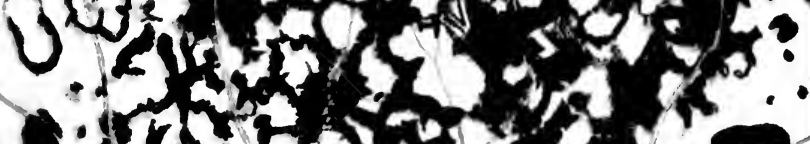
- derarates a

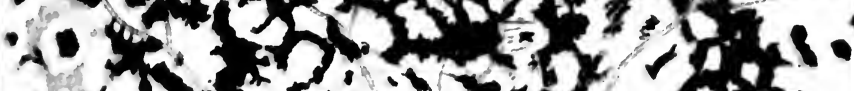

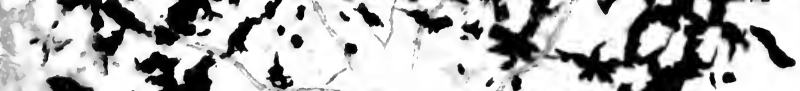
$8 x^{2}=C_{0}+5-21$

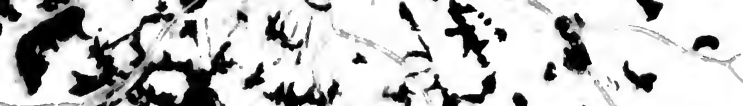

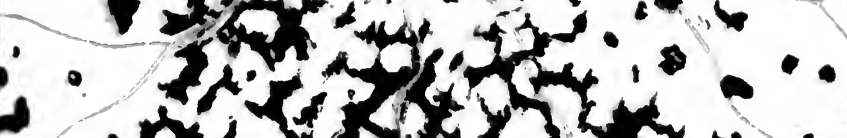

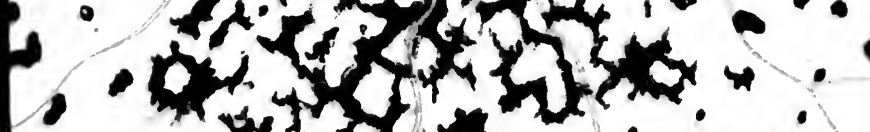

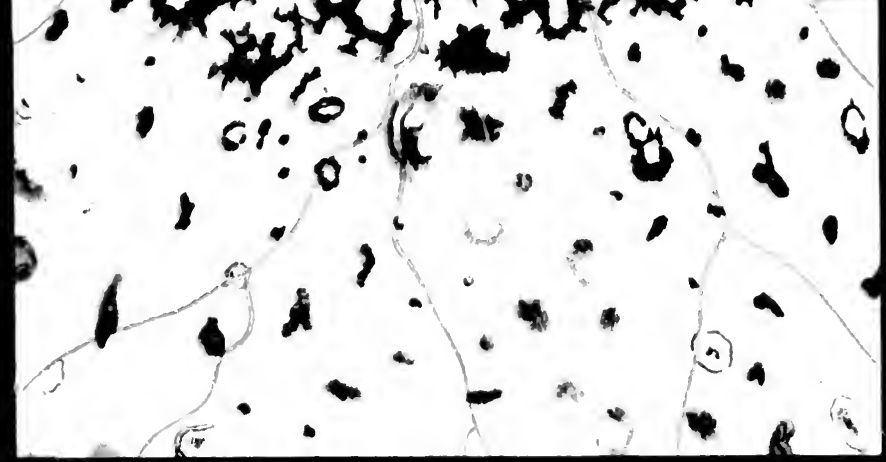






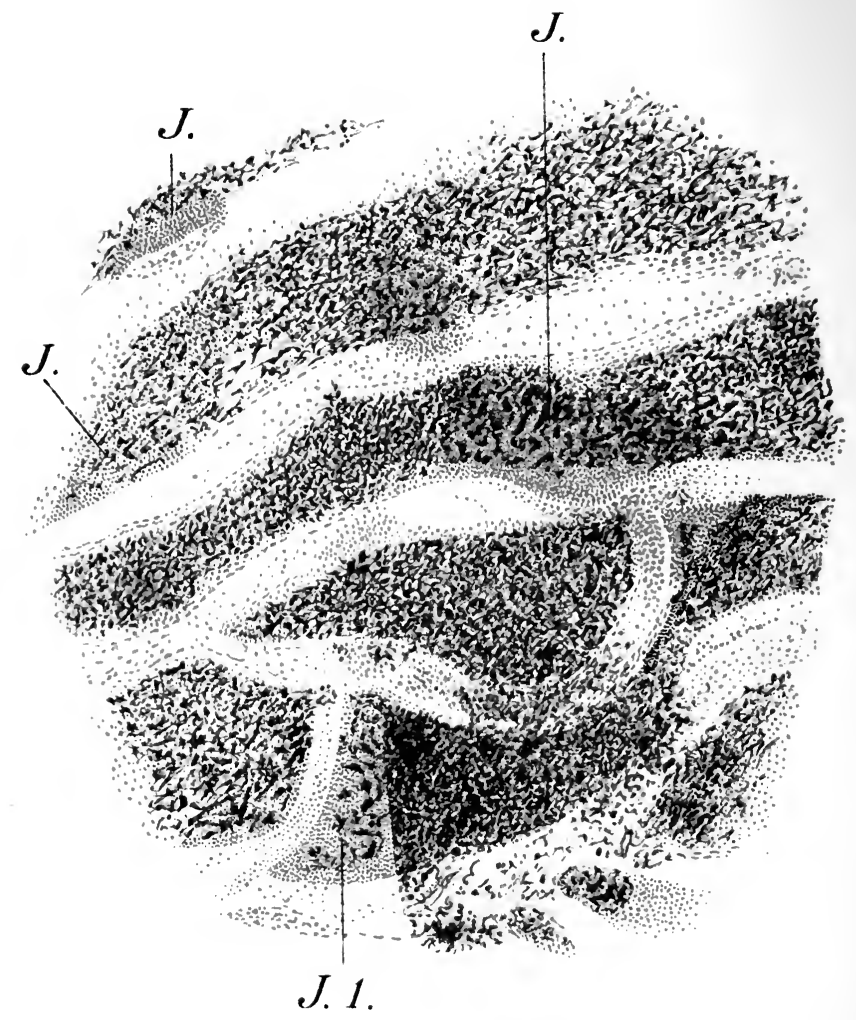

Fig. 70. 
Fig. 70. Areas of Infiltration $\left(J, J, J_{1}\right)$ in the Choroid in Disseminated Choroiditis, seen from above, so that the choroidal vessels (yellow) appear as in the ophthalmoscopic image, although magnified 78 times.-The stroma of the choroid between the patches is darkly pigmented (pigmented intervascular spaces). While, under normal conditions, comparatively few cell-nuclei are found in the vessels in these portions of the tissue, we find thick accumulations of them in this figure $\left(J, J, J_{1}\right)$ stained violet with hematoxylin. At the center of the section such an accumulation is seen around the blood-vessel. The inflammatory area at $J_{1}$, bounded by a straight line, is obscured on the right by a small portion of retinal pigment-epithelium that adhered to the preparation, showing that an area of inflammation in the choroid may, under certain conditions, remain invisible behind the retinal pigment-epithelium, and become apparent only after it has reached a certain size or has existed for some time, and has caused the disappearance of this epithelium. If, however, the pigmentation of the epithelium is slight, an area of this kind will be seen earlier in the ophthalmoscopic image and appear gray or yellowish-gray. It may also happen that the pigment of the choroid itself obscures small areas of this kind, so that they are nearly or quite invisible, as is the case in certain portions of the present picture.

Magnified 78 times. 


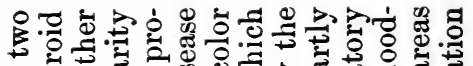
o 형

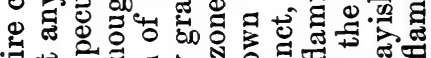
궁

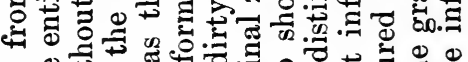

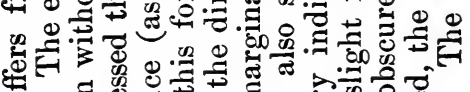

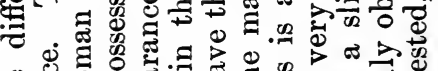

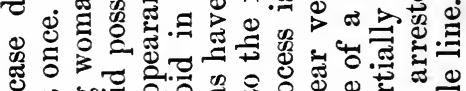

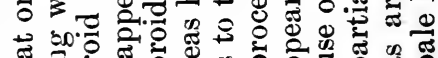

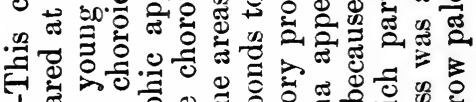

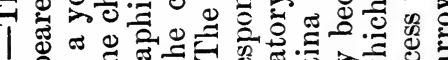

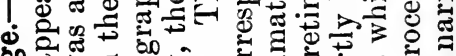

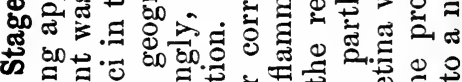
क人.

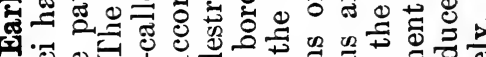

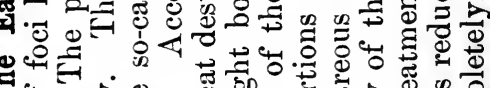
=

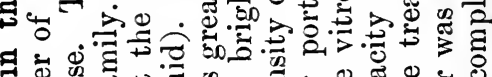

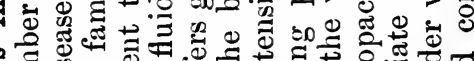

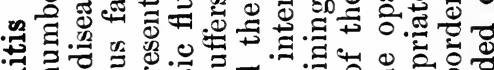

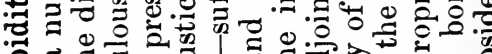
\%

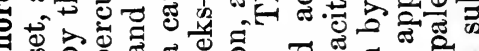

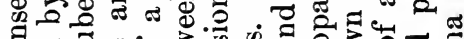

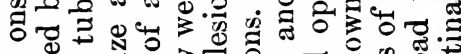

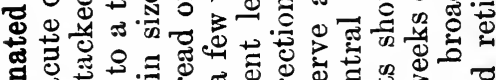

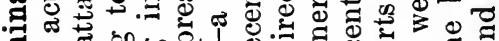
व .

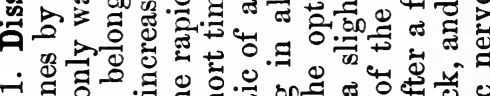

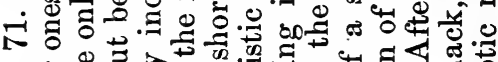

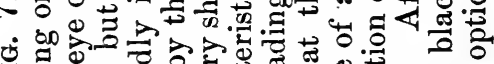

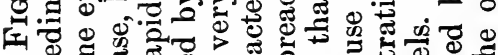

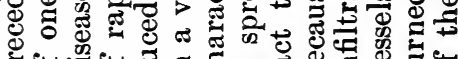

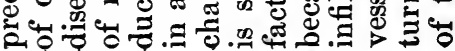




$$
[
$$





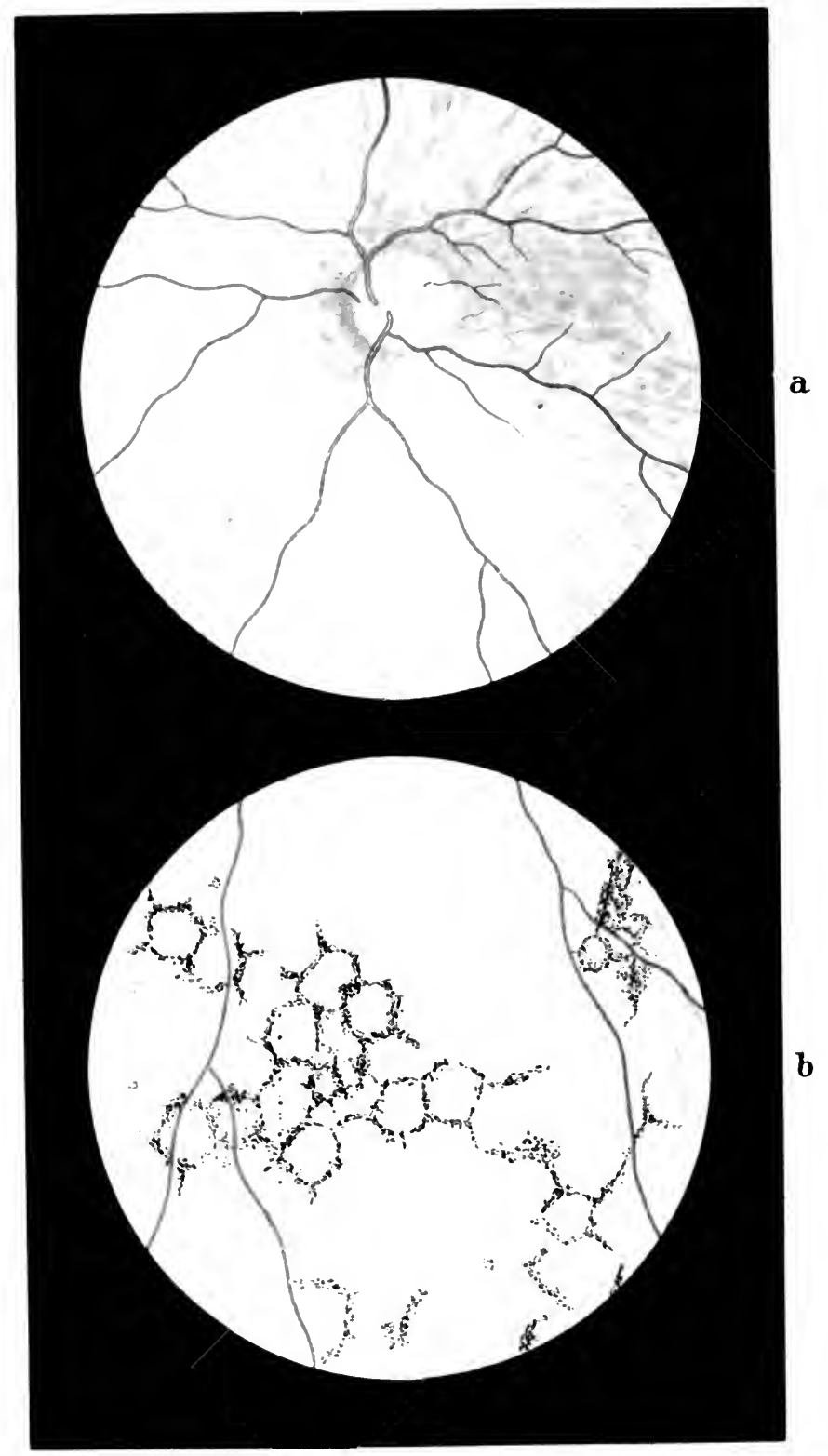

Fig. 72. 
Fig. 72, a. Hyaline Bodies (Drusen) in the Vitreous Layer of the Choroid.-The patient, a woman sixty-one years of age, had the same condition in the eye-ground of the other eye, although vision was normal on both sides. The patches are recognized as drusen of the vitreous layer by their position behind the retinal vessels, their yellowish-white, somewhat shining color, and their somewhat circular outline. They are usually found in the neighborhood of the papilla. The anatomic appearance of these structures is shown in Fig. 82, $a$.

FIG. 72, $b$. Senile Pigmentation of the Retina.-The picture was taken from a man seventy-six years old, who, like many persons of advanced age, showed pigmentation at the periphery of the retina in both eyes. The pigmented patches appear in the form of fine dots irregularly distributed or in the form of lines frequently forming more or less distinct pentagons and hexagons. İ have seen these peculiar figures in other similar cases. The visual field in this man was normal as regards the external borders, though there was some contraction of the color-field. There was no nyctalopia. Visual acuity was impaired by beginning cataract. It may, however, be quite good in spite of the retinal pigmentation, as the central portion of the retina usually escapes.

It is not uncommon to find drusen of the vitreous layer in combination with this form of retinal disease.

The pentagonal and hexagonal figures may possibly be explained by assuming that each figure contained within the dark dotted lines corresponds to a vascular or lymphatic nutritional region belonging to the retina or choroid, although this requires verification by more accurate anatomic study.

This senile pigmentation more frequently appears in the form of irregular figures and patches, as shown in the upper right-hand portion of the picture. 


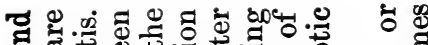

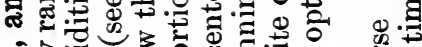

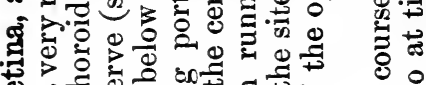

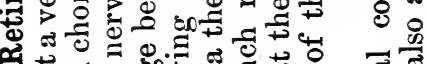

ศ

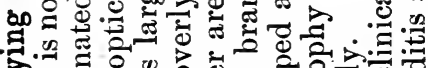

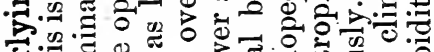

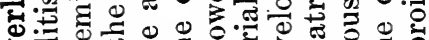

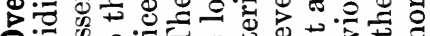

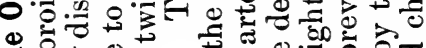

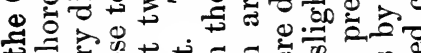

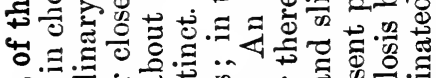

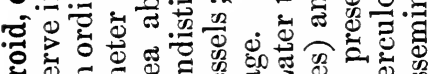

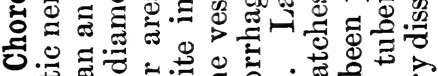

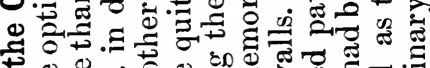

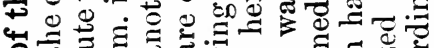

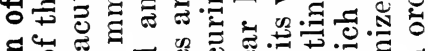
궁

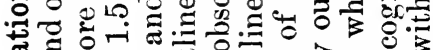
๙ हु

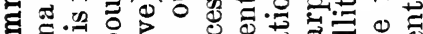
สิ. फ

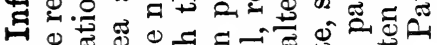

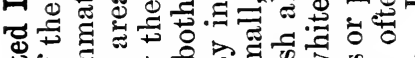

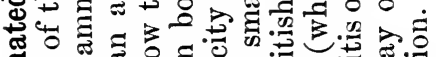

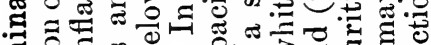

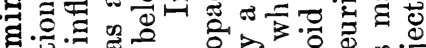

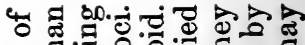

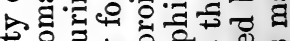

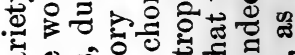

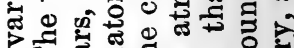
E. ⿷匚⿱

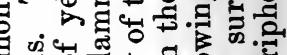

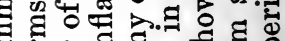

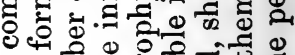
है 氠

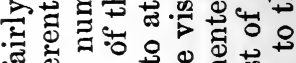

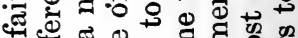
๘染

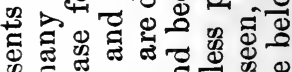

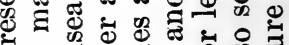

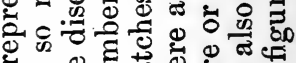

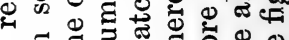

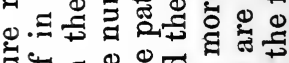

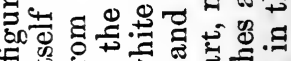
.

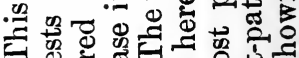

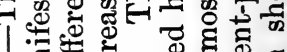

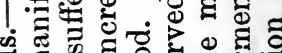
๘

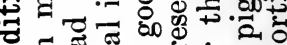
융로

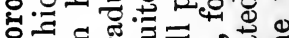

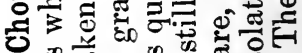
出

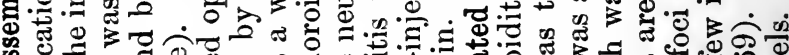

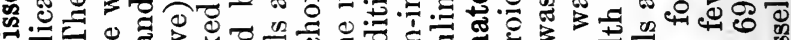

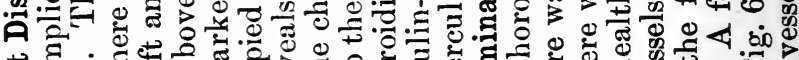

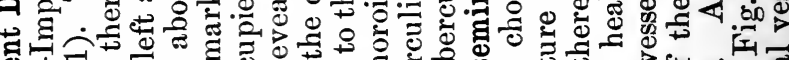

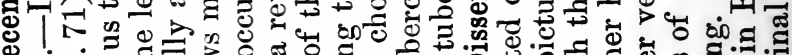

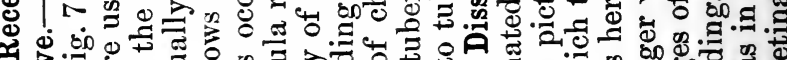
ن य

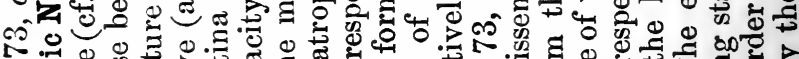

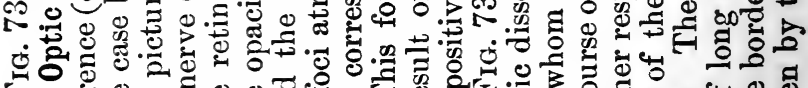

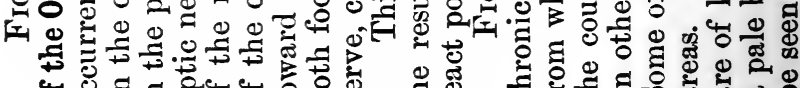

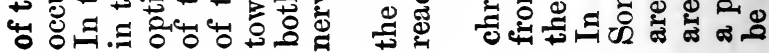




$$
0
$$





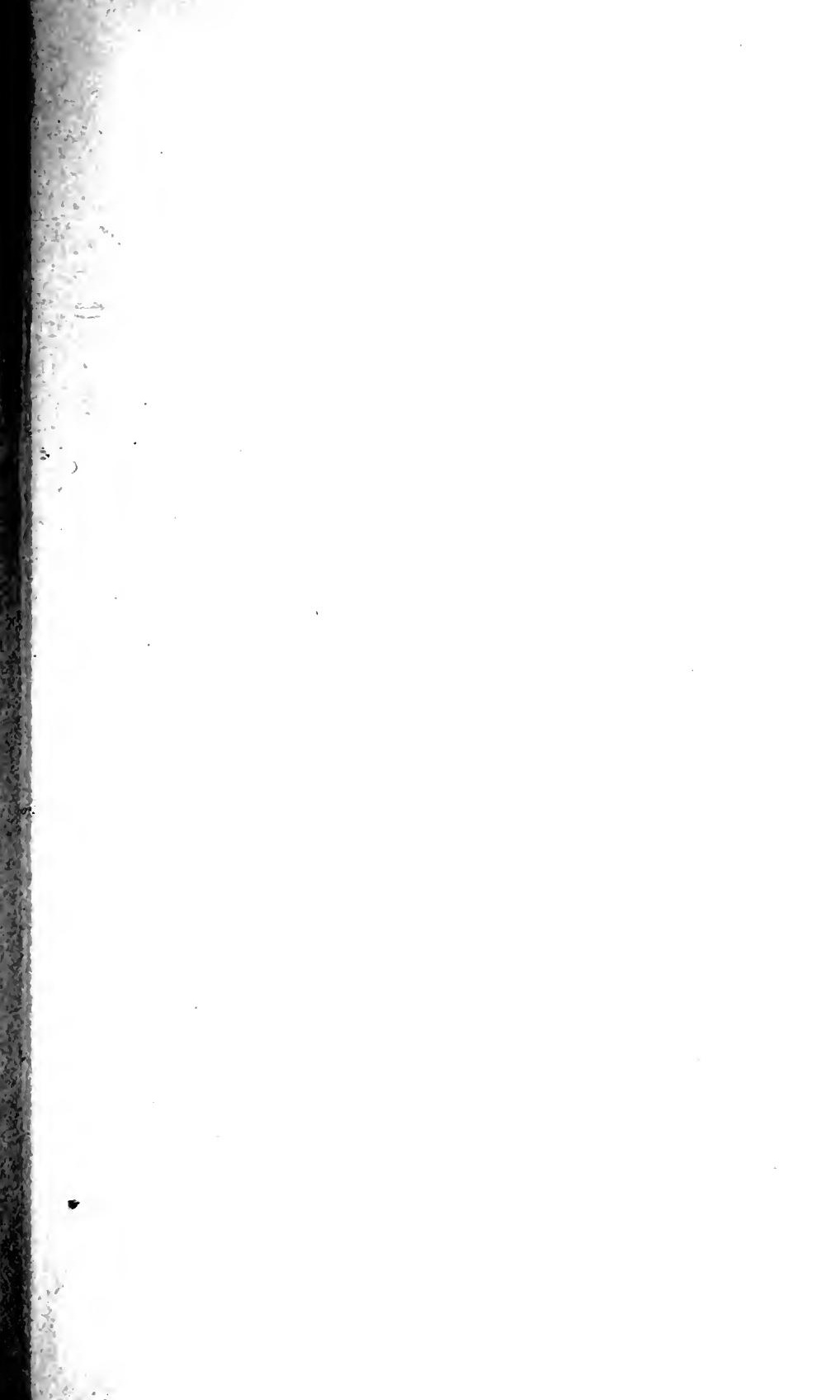




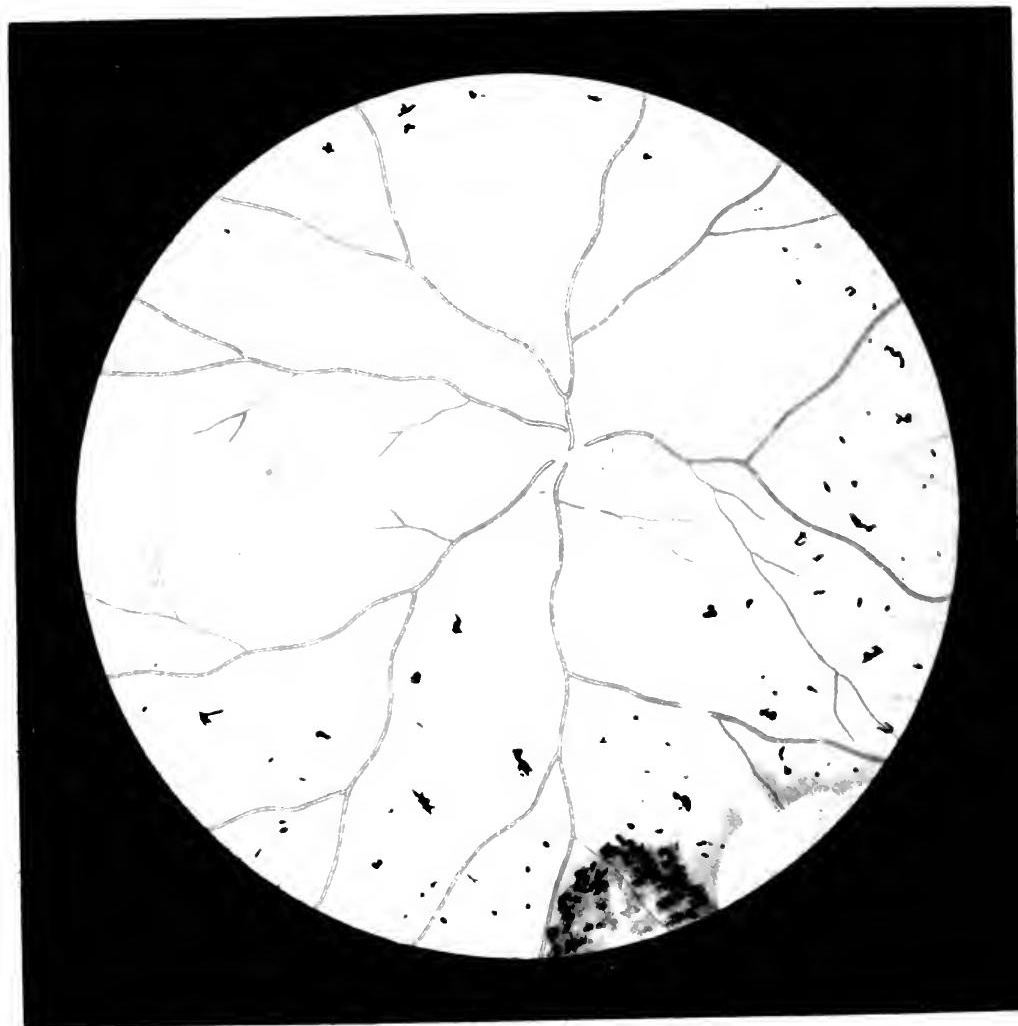

Fig. 74 . 
Fig. 74. Chronic Disseminated Choroiditis and Secondary Pigmentation of the Retina due to the Presence in the Eye of a Particle of a Metal Cap (direct method).-While the child was pounding metal caps a small piece of copper penetrated the sclera of the right eye, close to the inner corneal margin, and became surrounded by an exudate in the form of a grayish-white hemispheric mass (like the one seen in Fig. 60), projecting outward and downward in front of the equator into the anterior portion of the vitreous. In the neighborhood of the exudate a retinal detachment occurred which later disappeared. Then the exudate surrounding the foreign body became diminished in size. For a long time, however, a linear patch of exudate was seen passing through the vitreous toward the optic nerve. The exudate itself and the linear patch are seen in the lower right-hand portion of the picture, which was made a year and a half after the injury. In the meantime a number of patches, ranging in color from yellowish-red to black, appeared over the entire visible portion of the eye-ground, especially in the inner lower quadrant. Immediately after the receipt of the injury a slight optic neuritis, and later a macular disease in the form of minute dotting (shown in Fig. 53, $b$ ), made their appearance. At the time the picture was taken the optic nerve had somewhat regained its normal condition, but the macula still showed marked changes, especially the dotting at the periphery. The pigment-patches resemble those seen in pigmentary degeneration of the retina, and probably lie for the most part within that membrane.

During the twenty years following the injury the pigmentation of the retina increased considerably, especially at the periphery. Vision is still $\frac{1}{3}$.

This pigmentary degeneration is also shown in Fig. 46, a, which was taken from a similar case. 
Fig. 75. Miliary Tubercles in the Choroid in Acute Miliary Tuberculosis, an appearance not rarely seen in this disease shortly before death.-The circular patches, some of which tend to coalesce, are not sharply defined and, while they are small and still partially covered by pigmentepithelium, show gradations in color from grayish-white to yellowish-white and, finally, yellowish-red. The larger nodules occasionally project somewhat and force the retina outward, so that they impart a corresponding bend to any retinal vessel that may pass over them. Miliary tubercles are often difficult to distinguish from the lesions of disseminated choroiditis, as, for instance, the form shown in Fig. 68.

The anatomic features of this disease of the choroid are more fully shown in the transverse sections of Fig. $82, b$ and $c$. 



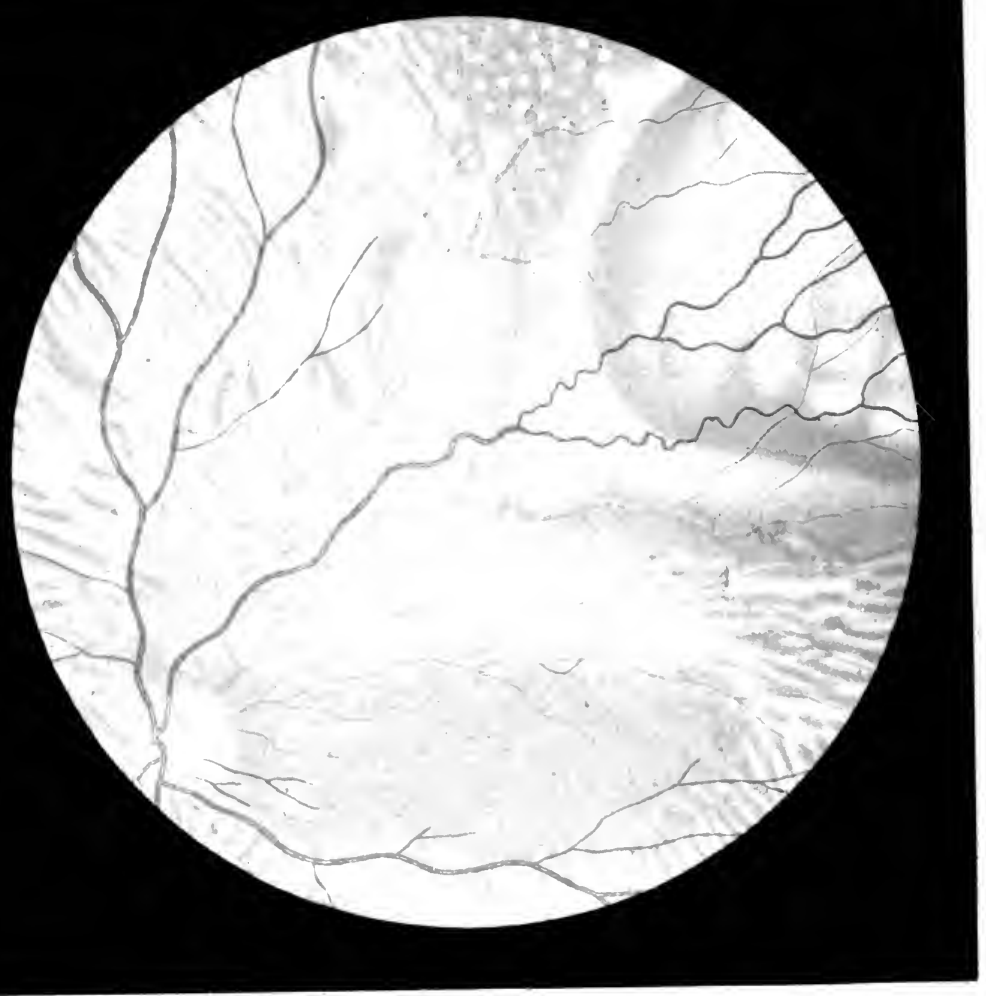

Fig. 76 . 


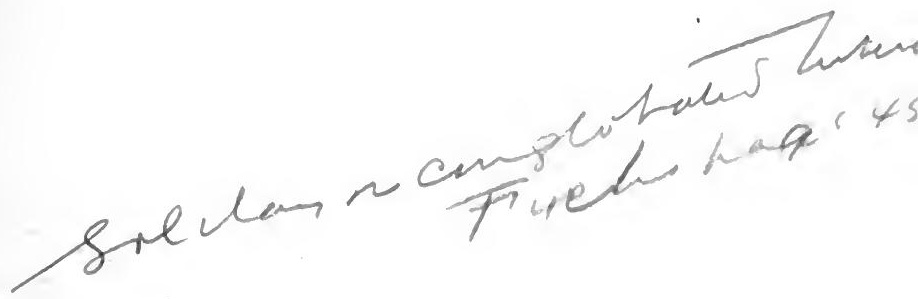

Fig. 76. Chronic Tuberculosis of the Choroid (direct method).-The tuberculous proliferation, consisting of a great number of closely aggregated tubercle-nodules-so-called conglobate tubercles-forms a slowly growing tumor which, even with the ophthalmoscope, is seen to be composed of grayish-red nodules. The tumor lies at the upper portion near the periphery; its anterior border cannot be seen with the ophthalmoscope, while its posterior border is surrounded by an irregular and ill-defined pale border of choroid which is of some diagnostic importance, as it shows inflammation and corresponding loosening of the pigment. In addition, a number of small disseminated white and yellow choroiditic spots are seen near the edge of the border, giving one the distinct impression that the tumor is setting up inflammation in its immediate neighborhood, a phenomenon which is constantly observed in tuberculous proliferations and distinguishes them from sarcoma, for instance. We also see that the optic nerve is inflamed, that is to say, reddened, and not sharply outlined, although this may be due to the fact that the boy was suffering from a complication of conglobate tubercles and miliary tuberculosis of the brain. (This case, in which my teacher [Horner] for the first time diagnosed conglobate tubercles of the choroid in man with the ophthalmoscope, I have described more in detail, both clinically and anatomically, in Gräfe's Archiv, vol. xxv.). 
FIG. 77. Sarcoma of the Choroid.-The round, bluish-gray tumor with a somewhat mottled surface, seen in the left half of the picture, projected into the vitreous from the region of the equator and obscured one-half of the optic nerve. In examining by the direct method, if the surgeon moves his head to and fro, he will see the edge of the tumor being pushed over toward the optic nerve, showing that the edge of the neoplasm does not lie upon the optic nerve, but at some distance in front of it, corresponding to the curve of the roundish nodule. The neoplasm is covered by the retina, as we see by the vessels which traverse it. The course of the vessels differs somewhat from that in the normal retiua, being slightly more tortuous. The sarcomatous nature of the tumor is recognized by the dark color of the entire mass and the somewhat fainter mottling of the surface. A simple detachment of the retina would present folds. At the lower border of the picture there is a simple retinal detachment such as frequently accompanies tumors of this kind. The rest of the eye-ground is normal. In both this and the following case we have to deal with a pigmented sarcoma.

Fig. 83 shows the anatomic relations of this important form of tumor, which must be differentiated from chronic tubercular proliferation (see remarks accompanying Fig. 76) and from glioma of the retina. The latter tumor is rarely secn with the ophthalmoscope (see Fig. 64, b). It has no pigment and its surface frequently presents hemorrhages. It occurs only in youthful individuals, while sarcoma of the choroid, on the other hand, is rarely seen before the twelfth year.

A (moderately pigmented) choroidal sarcoma may be simulated by an invagination of the globe by a tumor growing on the outside. This may occur when the tumor presses upon the side of the eyeball, and thus gradually causes the wall to bulge in the equatorial region, without penetrating into the interior of the eyeball. I have known this to happen in two cases of carcinoma in the anterior portion of the orbit, originating from the upper jaw and the cavity of the nose.

Finally, sarcoma must be differentiated from the so-called phantom tumors which probably originate in the retina and produce one or more spherical prominences in the anterior portion of the bulb. They are probably due to cysts produced by senile degeneration of the retina, for they not infrequently disappear in a short time without leaving a trace. They are usually seen by direct inspection or, after the pupil has been dilated, by lateral illumination. 
Fig. 78. Sarcoma of the Choroid.-In this case the pigmented mass is considerably larger than in the previous one. The tumor is hemispheric and the posterior border nearest the optic nerve is not seen, because it is obscured by the overhanging portion of the neoplasm. Hence the retinal vessels disappear for a time and reappear again on the surface of the tumor, where their course is irregular. The pigmentation of this tumor is a little less intense, and it therefore appears paler than the one in the preceding figure. 
FIG. 79, a. Recent Disseminated Choroiditis.-The areas of infiltration $(J J)$ seen here in transverse section, instead of from above, as in Figs. 67 and 70 , lie between, and in some cases in front of, the vessels which are also seen in transverse section, and are partially filled with blood.

FIG. $79, b$. Later stage of the disease when the retina is involved, that is to say, has already undergone connective-tissue degeneration wherever it comes in contact with a choroidal focus, and thus becomes involved in the disease. In places the retina is reduced to a mere connective-tissne membrane into which the pigment from the pigment-epithelium is beginning to penetrate. The latter is engaged in proliferation; some of the cells have lost their pigment and in some places the epithelium has entirely disappeared. Underneath, throughont the entire preparation, the vitreous layer of the choroid $(L . v$.$) is seen. In the choroid we see a$ few blood-vessels filled with blood, in transverse and longitudinal section. On the whole, however, the growth of connective tissue and scartissue is indicated by the fact that the vessels and the pigment are less prominent in certain places. The round-celled infiltration at $J$ is the result of an exacerbation in the inflammatory process. In some places the retina and choroid are evidently adherent: the open spaces between these adhesions, which were in part filled with exudate and pigment, have, for the most part, lost their contents in the preparation.

FIG. 79, $c$. In this preparation we do not see any recent inflammatory infiltrations. The retina and choroid are thinned, closely adherent, and reduced to mere cicatricial tissue. In places the pigment-epithelium has undergone marked proliferation and projects into the retina (secondary degeneration of the retina, ef. Fig. 43). In other places the pigmentepithelium is altogether wanting. In the choroid almost all the vessels have disappeared and the pigment is present only in places. Wherever the pigment and the pigment-epithelium overlying it are wanting (as, for instance, at the left extremity of the figure), the sclera shines through and thus forms the white spots characteristic of disseminated choroiditis, while the accumulations of pigment $(P)$ produce the black spots seen in the ophthalmoscopic image.

The three figures are magnified 78 times. 

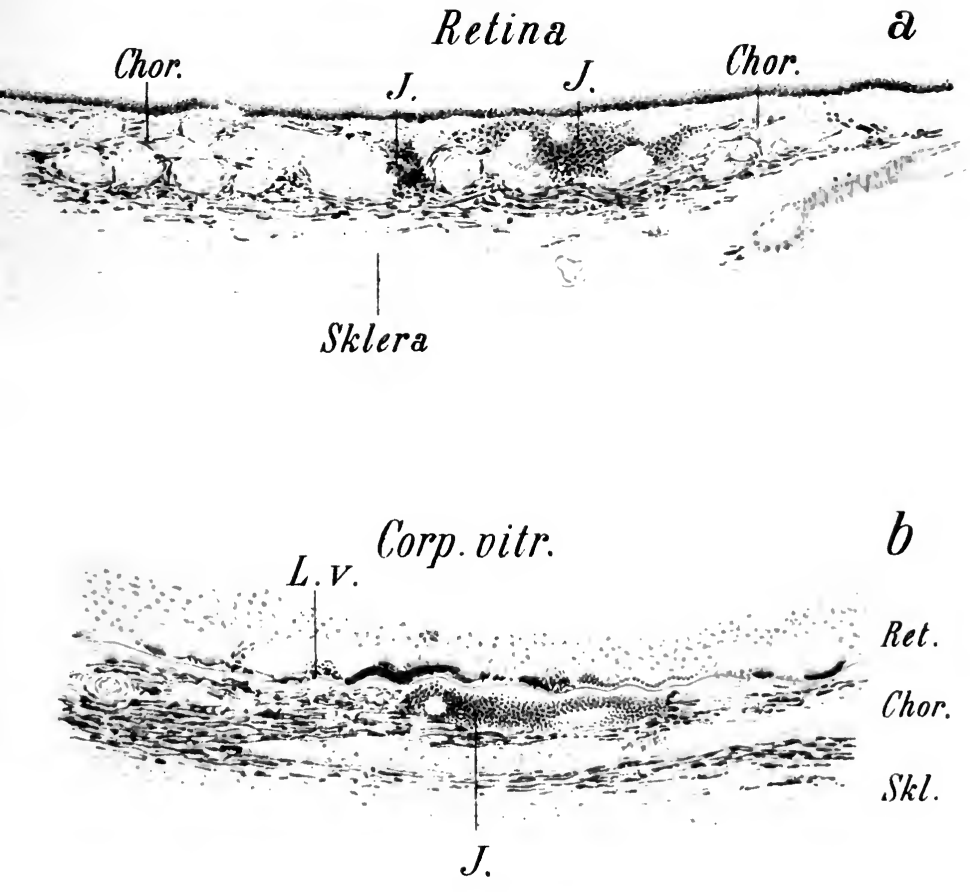

c

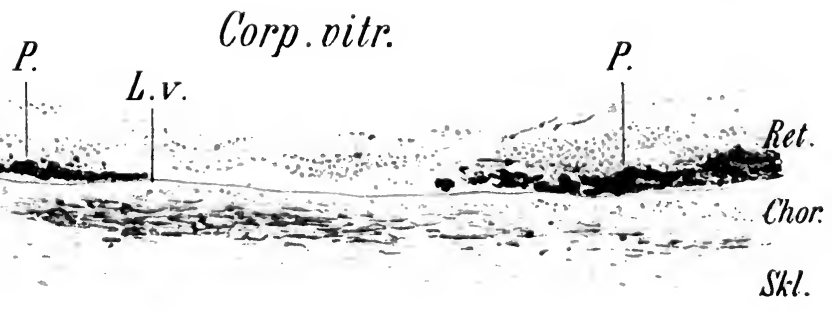

Fig. 79. 

Frg. 80. Changes in the Choroid due to Violent Contusion of the Eye; Lacerations of the Choroid.-'The eye was severely contused by the paper wadding from a blank cartridge. After the blood which had been poured out into the anterior chamber and into the vitreous disappeared, a large area to the nasal and lower side of the optic nerve and half of the nerve itself were seen to be covered by a whitish membrane, evidently consisting of connective tissue, completely obscuring the retinal vessels and the borders of the optic nerve, as shown in the figure. The outline of this membrane consists of a series of bizarre curves and in places is edged with black. To the nasal side there are five variously sized linear lacerations in the choroid, characteristically arranged in concentric curves parallel with the equator, their white color being due to the fact that the sclera shows through the tears in the choroid. They are sharply outlined and in places slightly edged with black. The retinal vessels continue their course over the lacerations without interruption. The area between the optic nerve and the macula is finely dotted. The rest of the eye-ground is normal. 
Fig. 81. Sclerosis of the Choroidal Vessels; Disseminated Choroiditis and Secondary Pigmentation of the Retina.This figure is taken from an unusually well-developed case, though we not infrequently see much less pronounced examples in which the alterations are confined to a small portion of the eye-ground. The most important features of the disease are the arteriosclerosis of the choroidal vessels and the atrophy of the retinal pigment, so that the choroid becomes very distinct and the choroidal vessels, the walls of which become white and opaque, appear almost white on a dark background, instead of presenting the usual appearance of a red vascular plexus. These changes in the bloodvessels are most marked at the anterior pole around the optic nerve and gradually decrease toward the periphery. On the outskirts of the area of greatest arteriosclerosis are a few white atrophic patches in the choroid, some of them with a pigmented border. We also see in this zone a few angular patches, consisting only of pigment, which are probably situated in the retina. The retina is not diseased and the walls of its vessels show no changes.

The experiments of Wagenmann have shown that disturbance of the nutrition of the choroid is followed also by atrophy and secondary pigmentation of the retina. 

Fig. 82. a. Hyaline Bodies (Drusen) of the Vitreous Layer (cf. Fig. 72).-The hyaline structures, stained violet with hematoxylin, are seen upon the vitreous layer surrounded for the most part by the cells of the pigment-epithelium. The choroid is normal. The retina in this case was detached and is, therefore, not seen in the figure.

Fig. 82, b. Miliary Tubercle of the Choroid (cf. Fig. 75). - As the section is near the equator and parallel to it, most of the choroidal vessels appear in transverse section. At the center of the tubercle there are a few giant-cells, The retina is not shown in the drawing. In mounting the preparation the choroid became separated from the sclera and the interval is in part filled with the layers of the suprachoroidea.

The patient died of miliary tuberculosis.

Frg. 82, c. Large Tubercular Growth in the Choroid composed of Several Nodules: $V$, transverse section of a choroidal vessel; $K$, caseous portion of the tubercle; R.R., giant-cells.

The three figures appear magnified 30 times. 
Fig. 83. Meridional Sections through Eyes with Choroidal Sarcoma, stained with hematoxylin and eosin (life-size).In $a$ there is between the retina and the tumor a layer of amorphous exudate similar to the exudate beneath the rest of the retina which is detached.

In $b$ the tumor, except for its posterior portion, is covered by the retina; while in $c$ the retina invests the entire surface of the tumor.

In none of the three cases did the tumor break through the sclera.

In $d$ is shown the microscopic structure of a pigmented sarcoma of the choroid, which is seen to be composed principally of spindle-shaped cells containing more or less pigment, though in places the pigment is entirely absent.

Magnified 112 times. 

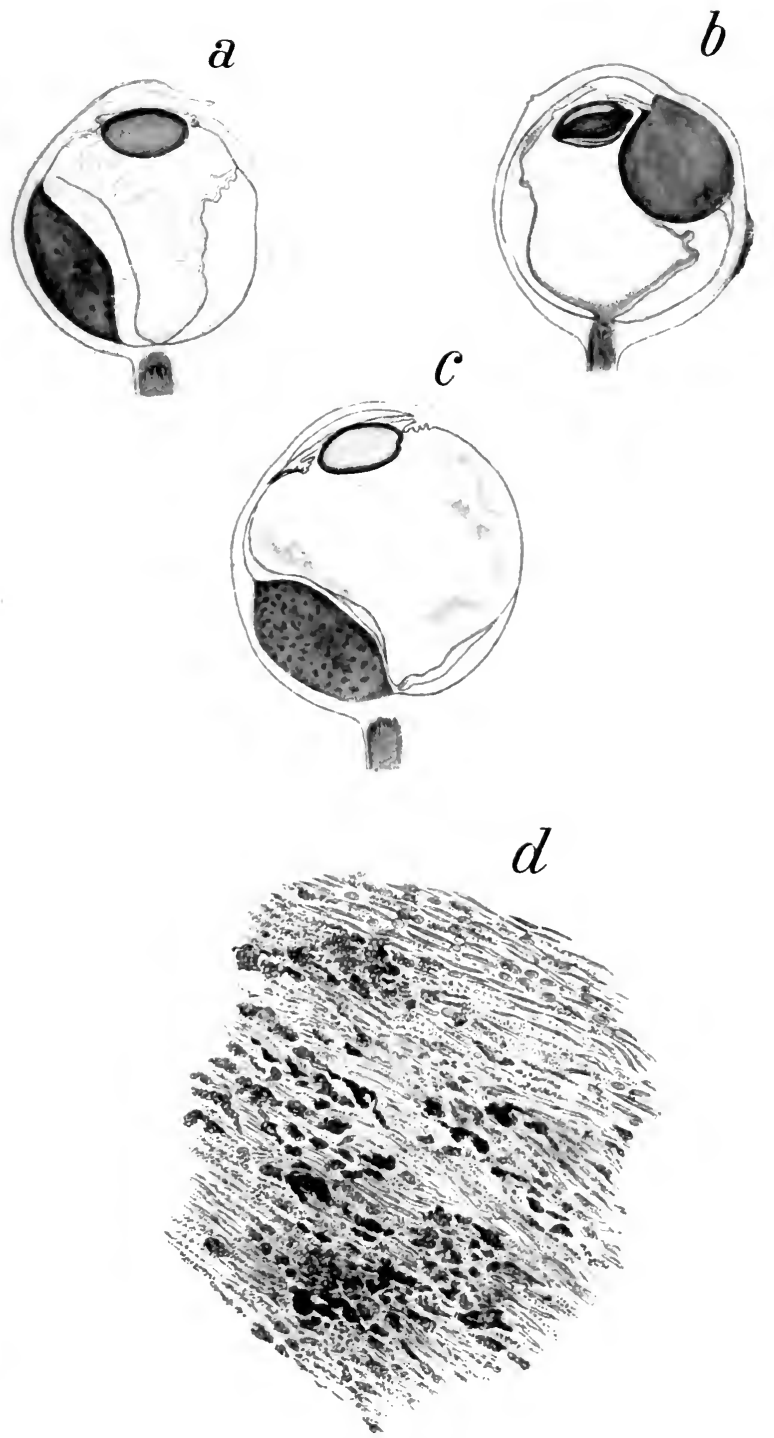

Fig. 83. 




$$
\theta
$$




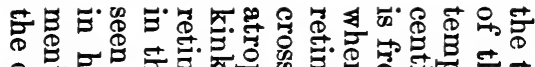

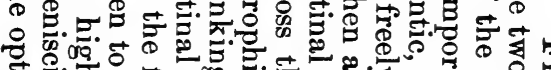

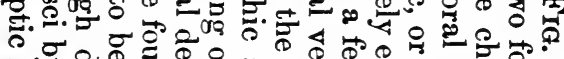

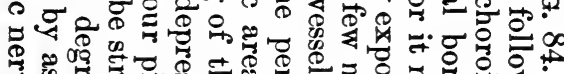

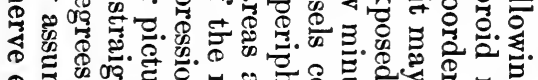
B

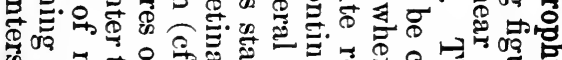

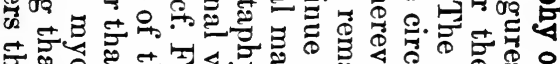
ब

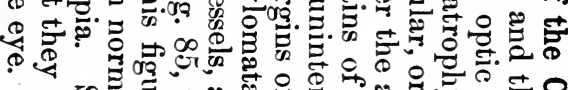

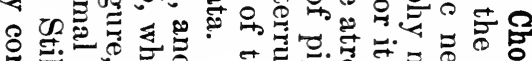

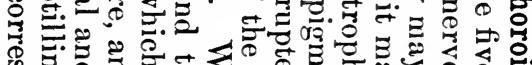

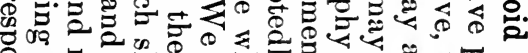

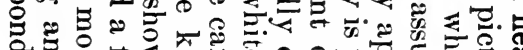

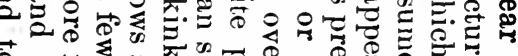

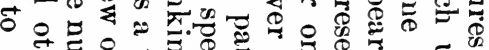

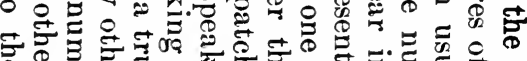
के

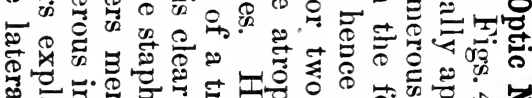

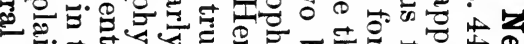

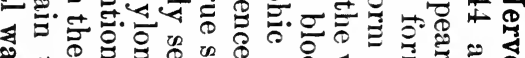

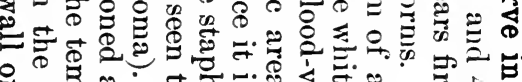

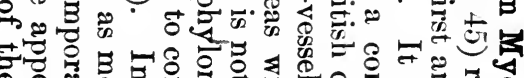

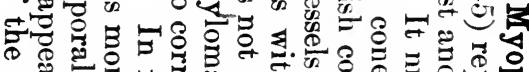

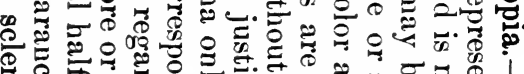

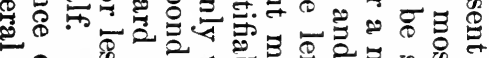

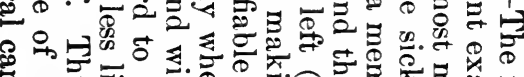

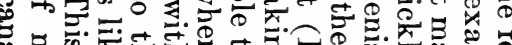

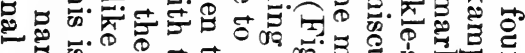

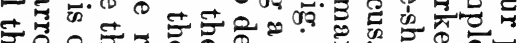

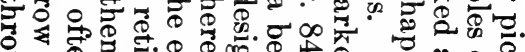

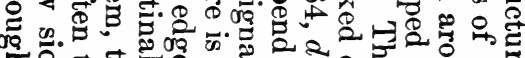

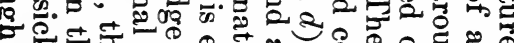

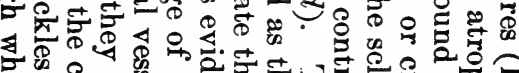
E.

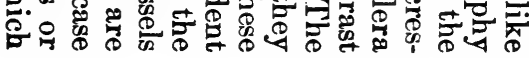


Fig. 85. True Staphyloma in a Highly Myopic Eye (first described by Weiss).-While the pale atrophic "sickles" or "menisci" at the temporal border of the papilla or the circular atrophy of the choroid around the papilla when moderately large, practically never, and when quite large very rarely, show any staphylomatous bulging, and therefore have no just claim to this obsolete designation, this is not the case in a true staphyloma, such as we have represented here, which is usually met with only in high grades of myopia (above $20 \mathrm{D}$ ) and is accordingly uncommon.

In this case we see, especially to the nasal side of the papilla, the distinct border of an excavation, and the bloodvessels as they pass over it are deflected. The edge of the excavation is recognized either by a more or less marked shadow or by a more or less distinct gray line running along the summit. The curve varies considerably in size, that is to say, it may form a greater or larger segment of a circle or of an oval. Occasionally the edge of the staphyloma is circular and surrounds the entire posterior pole, but even in such cases it is always most pronounced to the nasal side of the optic nerve. The edge of the staphyloma is best seen by parallactic dislocation, even when it is difficult to see it with the ophthalmoscope.

Fig. 85 shows, in addition, three atrophic sickles near the papilla over which the vessels pass without undergoing deflection. We also note that the course of the retinal vessels is characteristic of a high degree of myopia : the main trunks, instead of passing upward and downward, extend more to the temporal side. The posterior pole (macula and surroundings) shows a decrease and loosening of the pigmentation, which is also characteristic of a high degree of myopia. In this patient I removed the myopia of $30 \mathrm{D}$ by an operation (discission of the lens), and the eye became almost emmetropic with good vision. 


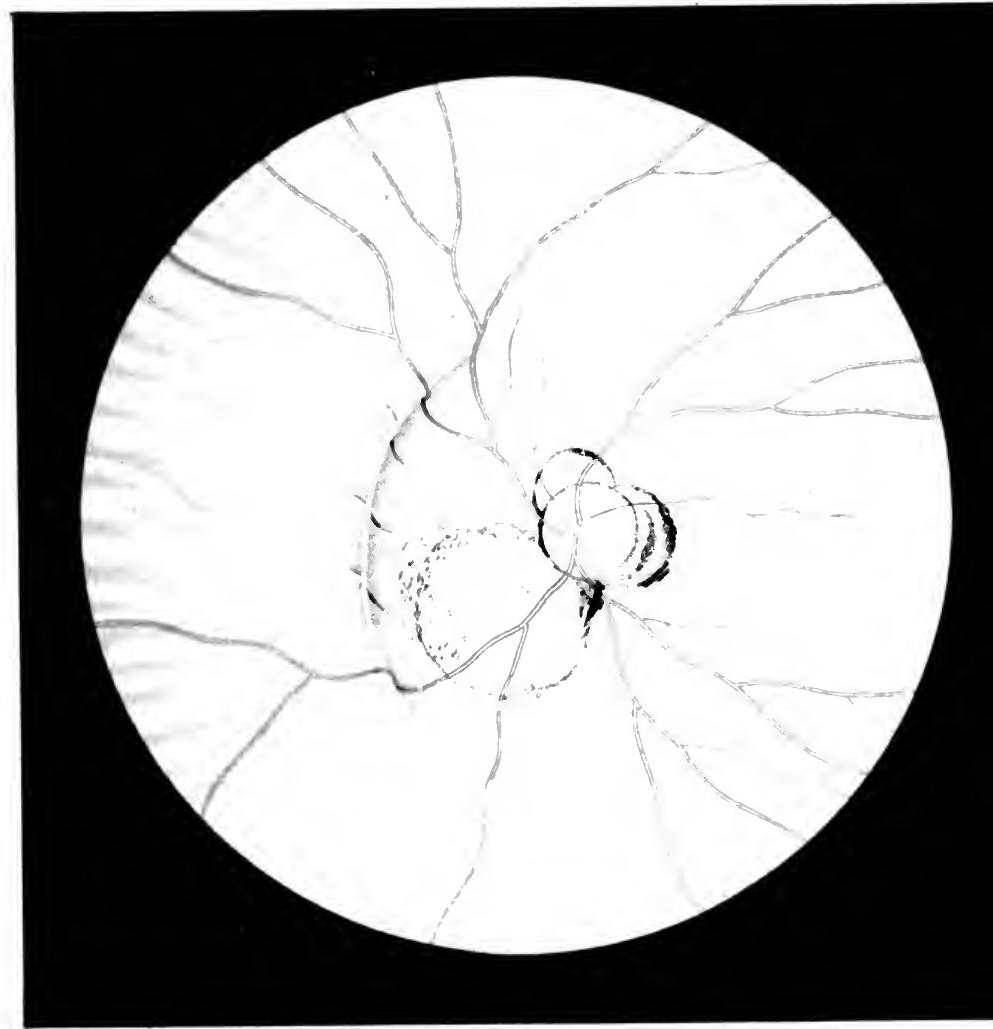

Fig. 85 . 




$$
9
$$




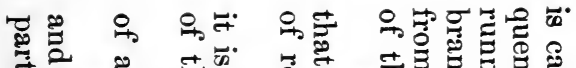

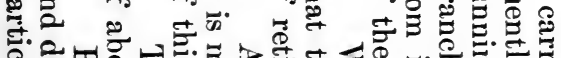

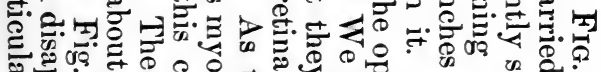

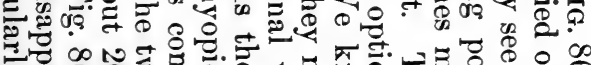

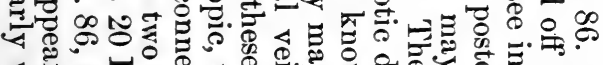
₹

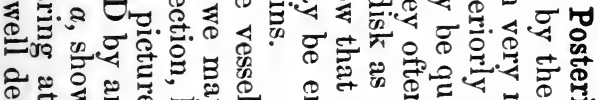

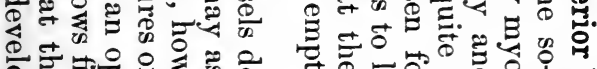

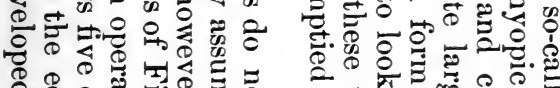
\&율

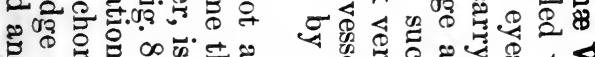
:

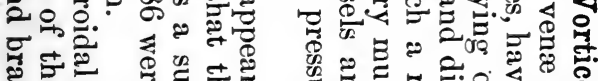

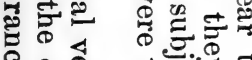
कㅇ. 당. 哈

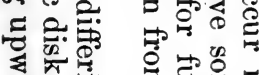

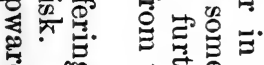

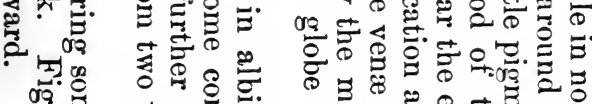
93

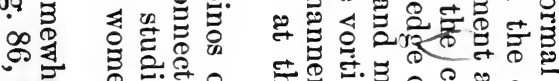

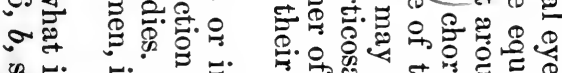

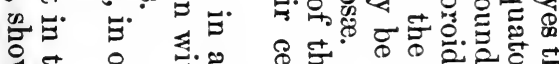

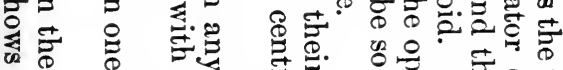

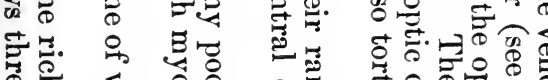

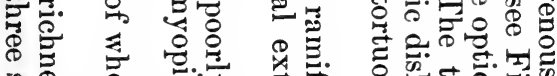

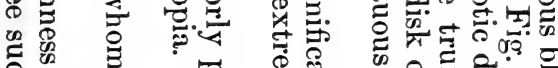

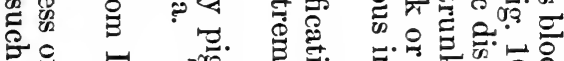
क

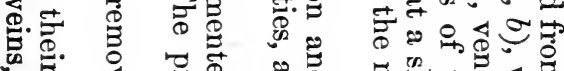

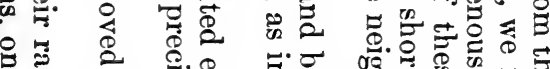

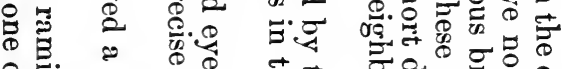

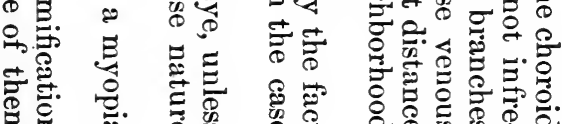

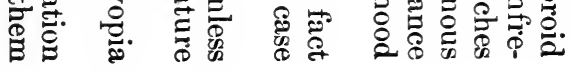


FIG. 87. Pupillometer.-Since it is quite easy in comparing two eyes to estimate a difference of a few centimeters in the width of the two pupils, it would seem to be wise to adopt a similar method of measuring the pupil in daily practice and to determine the width of the pupil by comparing it with a painted picture of a pupil. A number of black disks arranged in a perpendicular row are held near the eye and the disk corresponding to the pupil is then picked out. This method is sufficiently accurate for ordinary purposes, as I have found in my own practice.

One of the strips on the opposite page should be cut out and attached to the millimeter measure which is used for measuring the pupillary distance in ordering glasses, or it may be pasted on a strip of cardboard. The two other strips will serve to renew this simple instrument. 
Tab. 87.

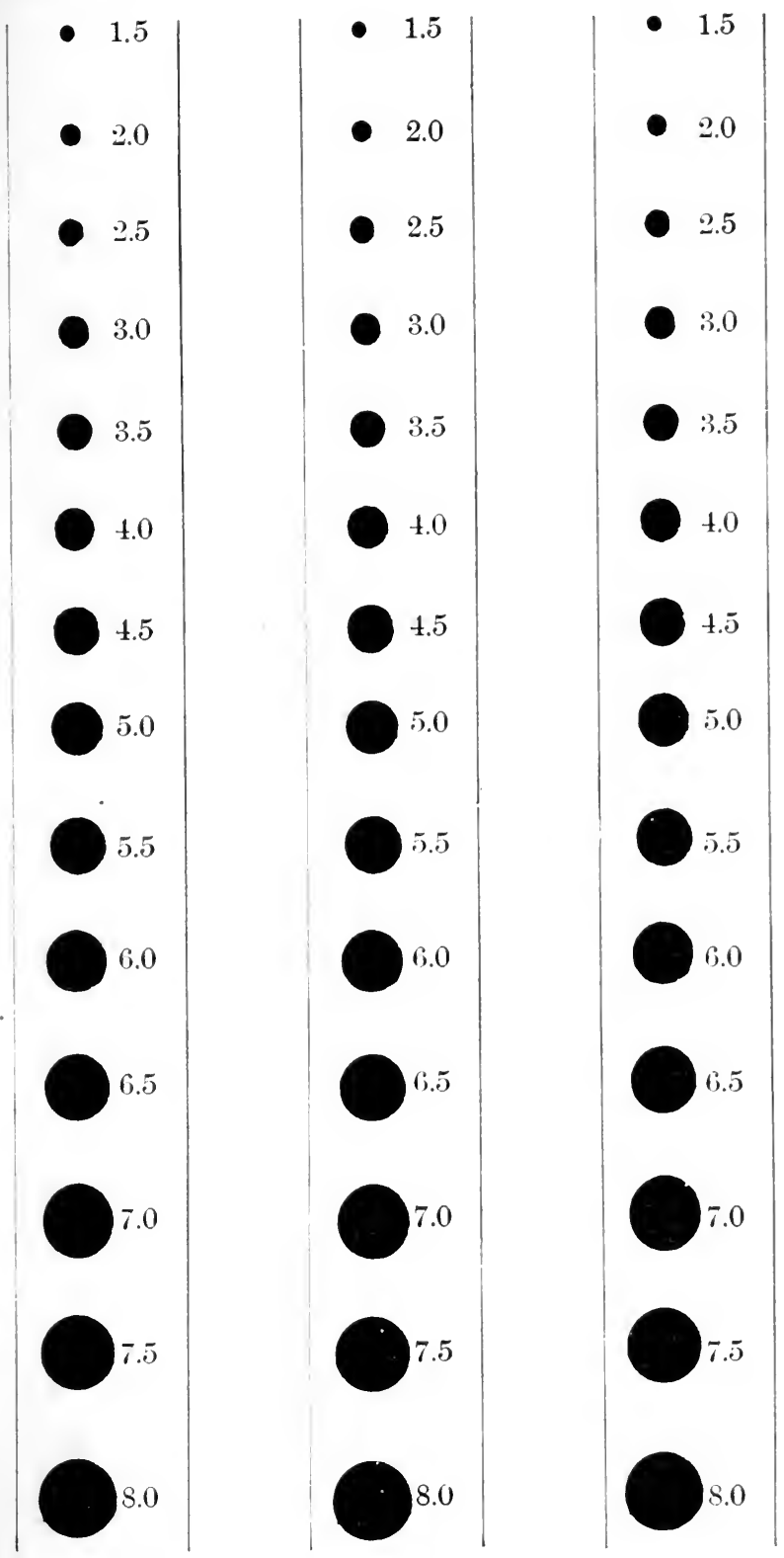





\section{INDEX.}

Accommodation in direct method, /Choked disc, Figs. 13, 13, $a, 15$ 23

Air-bubble in vitreous, Fig. 55

Albinismus, Fig. 10, $b$

Albuminuria, retina and optic nerve in, Figs. 25, 28, $a$

Albuminuric disease of eye-ground, Fig. $28, b$

Anemia, pernicious, eye-ground in, Fig. 31

retina in, Fig. $67, a$

Anterior chamber, angle of, after glaucoma, Fig. 24, $b$

Aortic insufficiency, arterial pulsation and, 86

Arteria centralis retinæ, 80

Arterial pulse, 82 aortic insufficiency and, 86

Arteries, 80

Astigmatism, 34

irregular, 37

measurement, 34

shadow-test for, 56

Atrophic menisci, Fig. 85

sickles, Fig. 85

Atrophy of choroid in myopia, Fig. 84

of optic nerve, Figs. 19, 58, I, $b$ due to intra-ocular tension, Fig. 22

gray, Fig. 20

partial, Fig. 21, $a$

total, Fig. $21, b$

of retinal vessels, Fig. 58, I, $b$

Berlin's opacity, Figs. 51, 53

Blood-vessels, 80

Brain-tumor, inflammation of optic nerve in, Fig. 13

Central artery, obstruction of, Fig. 32

vein, thrombosis of, Fig. 33, $b$

Chorioretinitis, Fig. 43

Choroid after contusions, Fig. 80 atrophy of, in myopia, Fig. 84 chronic tuberculosis of, Fig. 76 congenital defects of, Figs. 7-10 diseases of, Figs. 68-86

infiltration of, in disseminated choroiditis, Fig. 70

lacerations of, Fig. 80

miliary tubercles in, Figs. 75, $82, b$

phantom tumors of, Fig. 77

retina, and optic nerve, recent inflammation of, Fig. 73, $a$

and sclera, section through, Fig. 3

sarcoma of, Figs. 77, 78, 83

tubercular growth of, Fig. $82, c$

vitreous layer of, hyaline bodies in, Figs. $72, a, 82, a$

Choroidal pigmentation, 76

ring, 76

vessels, sclerosis of, Fig. 81

Choroiditis disseminata, Figs. 69, $73, b$

advanced case, Fig. 69

areas of infiltration in, Fig. 70

chronic, and pigmentation of retina, Figs. $43,74,81$

early stage, Fig. 71

inflammatory focus in, Fig. $67, b$

recent, Figs. $68,73,79$

postica, Figs. 44, 45

Cilioretinal vessel, 81

Coloboma choroideæ, Fig. 7

et nervi optici, Fig. 8, $a$

Color of eve-ground, 75

Colored illustrations in ophthalmoscopy, 15

Commotio retinæ, Figs. 51, 52

Congenital defects, Figs. 7-11

Contusion of eyeball, perforation after, Fig. 49 
Contusions, choroid after, Fig. 80 Corneal region, detection of opacities in, by transmitted light, 47

Cylindrical lens, 36

Diabetes, eye-ground in, Fig. 30

Direct method, determination of inequalities by, 45

enlargement of image in, 43

mirrors in, 73

of examination, 22

size of field of vision in, 38

Dislocation of lens, congenital, Fig. 11

Displacement, parallactic, 46

Disseminated choroiditis. See Choroiditis.

Donders on venous end-pulse, 84

Ectopia lentis congenita, Fig. 11

Embolism of superior temporal artery, Fig. 34

Emmetrope, examination by, 24

Emmetropic eye, direct examination, 24,27

examination in erect image, 27

Endarteritis, obliterating, of superior temporal artery, Fig. 34

Erect image, examinations in, 23

Erect-image method of examination, 22

Examination by indirect method, 40

by transmitted light, 47

in erect image, 23

of eye-ground, 13

ophthalmoscopic, 67

first step, 68

fourth step, 72

second step, 69

third step, 69

Excavation, physiologic, 77

Eyeball, contusion of, perforation of macula lutea after, Fig. 49

Eye-ground, albuminuric disease of, Fig. $28, b$

changes in, resulting from severe forceps delivery, Fig. 58, I, $a$ color, 75,76

colored illustrations of, 15

examination, 13

illumination, 20
Eye-ground in congenital syphilis, Figs. 41, 42

in diabetes, Fig. 30

in hereditary syphilis, Fig. 40

in leukemia, Fig. 65

in pernicious anemia, Fig. 32

irregularities on, determination, 45

normal, 73; Fig. 1

blonde, Fig. 4

dark, Fig. 5

observation of, 22

point of impact of foreign body on, Fig. 58, $a$

rules for observing, 32

FAR point, 26

Field of vision, size of, in direct method, 38

in indirect method, 42

Flame, size of, ophthalmoscopic field and, 39

Flux, intermittent, 83

Foreign body in vitreous, Fig. 53

detection of, by transmitted light, 50

Foveal reflex, 79

Gladcoma, angle of anterior chamber after, Fig. 24, $b$ atrophy of optic nerve from, Fig. 22

optic nerve in, Fig. 24, $c, d$

Glaucomatous excavation of optic nerve, Fig. 23

Glioma of retina, Fig. 66

HAAB's ophthalmoscope, 62

Heart-disease, arterial pulse and, 86

Helfreich on venous end-pulse, 84

Helmholtz's dises, 21

Hemorrhages into retina, Fig. 35

into vitreous, Fig. 35

Homocentric pencil, 34

Hyaline bodies in vitreous layer, Figs. 72, $a, 82, a$

Hypermetrope, examination by, 25

Hypermetropia, detection of, by transmitted light, 51

latent, 30

manifest, 30

total, 30

Hypermetropic eye, examination in erect image, 27

measurement, 29 
ILLUMiNation of eye under examination, 21

Image, enlargement of, in both methods, 43

Indirect method, advantages of, over direct method, 42

determination of inequalities by, 46

enlargement of image in, 44

examination by, 40

measurement of refraction by, 45

size of field of vision in, 42

Inflammation of optic nerve, Fig. 12

in brain-tumor, Fig. 13

in orbital tumor, Fig. 18

in syphilis, Fig. 16

intense, Fig. 17

Intermittent flux, 83

Introduction, 13

Irregular astigmatism, 37

JACKSON on shadow-test in astigmatism, 57

Jackson's ophthalmoscope, 65

Keratoconus, detection of, by Keratoscopy, 53 transmitted light, 52

LACERATION in retinal detachment, Fig. 62 of choroid, Fig. 80

Latent hypermetropia, 30

Lens, congenital dislocation of, Fig. 11

convex, in ophthalmoscopes, 62

in shadow-test, choosing of, 5.5 cylindrical, 36

detection of opacities of, by transmitted light, 48

Leukemia, eye-ground in, Fig. 65

Liebreich's ophthalmoscope, 66

Loring's ophthalmoscope, 63

Randall's modification, 65

\section{Macula, 78}

lutea, disease of, from age, Fig. 47

from blow, Fig. 48

from foreign body, Fig. 53

from myopia, Figs. 44, 45

from pressure and contusion, Fig. 54
Macula lutea, horizontal section of, Fig. 14

image in, 34

in disease from tumor, Fig. $50, a$

perforation of, Fig. 49, $a$

strippling of eye-ground in, 79 reddish-brown spot in, 76

Macular disease, Fig. 58, I, $b$ reflex, 78

Manifest hypermetropia, 30

Marginal reflex, 80

Marple's electric ophthalmoscope, 65

Medullated fibers of retina, Fig. 6

Menisci, atrophic, Fig. 85

Miliary tubercles in choroid, Figs. $75,82, b$

Mirror, concave, for shadow-test, 56

Mirrors in direct method, 39, 74

Myope, examination by, 24

Myopia, atrophy of, choroid in, Fig. 84

detection of, by transmitted light, 51

disease of macula lutea from, Figs. 44, 45

Myopic eye, measurement of, 26

true staphyloma in, Fig. 85

Nerve-fibers, medullated, in retina, Fig. 6

varicose, in retinitis albuminurica, Fig. 26, $b, c$

Neuritic atrophy, Fig. 19

Neuritis, optic, Fig. 12, $a$

and macular changes in tumor of cerebellum, Fig. 13, a

section through papilla in, Fig. 15

Neuroretinitis albuminurica, Fig. 25

specific, Fig. 16

syphilitic, Fig. 37

Normal eyc, section through angle of anterior chamber of, Fig. $24, a$

eye-ground, 74. See also Eyeground.

papilla, longitudinal section, Fig. 2

Obstruction of central artery, Fig. 32

of superior temporal artery, Fig. 34 
Opacities in corneal region, detection of, by transmitted light, 47

in refractive media, detection of, by transmitted light, 47

in vitreous, detection of, by transmitted light, 50

of lens, detection of, by transmitted light, 48

Ophthalmoscope, choice of, 58

convex lens, 62

description, 18,58

Haab's, 62

Jackson's, 65

Liebreich's, 66

Loring's, 63

Randall's modification, 64

Marple's, 65

Ophthalmoscopic examination, 67

first step, 68

fourth step, 72

second step, 69

third step, 69

field of vision, size of, 38

Optic disc, 77

nerve, 77

and retina in albuminuria,

Figs. 25, 28, $a$

atrophy of. See Atrophy.

choroid, and retina, recent in-

flammation of, Fig. 73, $a$

in orbital tumor, Fig. 18

diseases of, Figs. 12-24

glaucomatous excavation of,

Fig. 23

in glaucoma, Fig. $24, c, d$

inflammation of. Sce Inflammation.

left, viewing of, 70

malformation of, with congenital choroidal defect, Fig. 8, $a$ right, viewing of, 71

through ophthalmoscope, 76

neuritis and macular changes in tumor of cerebellum, Fig. $13, a$

Opticociliary vessel, 82

Orbital tumor, inflammation and congestion of nerve in, Fig. 18

\section{Papilla, 77}

normal, longitudinal section, Fig. 2

section through, in neuritis, Fig. 15

in papillitis, Fig. 15
Papillitis, Figs. 13, 15, 17 section through papilla in, Fig. 15

Parallactic displacement, 46

Pencil, homocentric, 35

Pernicious anemia, eye-ground in, Fig. 31

retina in, Fig. $67, a$

Phantom tumors of choroid, Fig. 77

Phenomena, pulsation, 82

Physiologic excavation, 77

Pigment, congenital absence of, Fig. 10,b

Pigmentary degeneration of retina, Figs. 38, 39

Pigmentation, choroidal, 76

Pigment-epithelium of retina, congenital defect of, Fig. $8, b$

Posterior venæ vorticosæ, Fig. 86

Pregnancy, hemorrhagic retinitis of, Fig. 63

Pulsation phenomena, 82

Pulse, arterial, 82, 87

venous, 84

Punctum remotum, 26

Pupil, cause of black appearance of, 18

Pupillometer, Fig. 87

Pupilloscopy, 53

RANDALL's modified Loring ophthalmoscope, 65

Red coloration of normal eye, 75

Reflexes on retina, 77

Refracting power, measurement of, 23

system of eye, 18

Refraction, points to be observed, 33

Schmidt-Rimpler method of measuring, 45

Refractive media, detection of, opacities in, by transmitted light, 47

surface with greater curvature in vertical than in horizontal meridian, 35

Retina, 77

and optic nerve in albuminuria, Figs. 25, 28, $a$

blood-vessels of, 80

choroid, and optic nerve, recent inflammation of, Fig. 73, a and sclera, section through, Fig. 3

glioma of, Fig. 66 
Retina, hemorrahges into, Fig. 35 in pernicious anemia, Fig. $67, a$ in retinitis albuminurica, Figs. $26,27,29$

in thrombosis of vena centralis retinæ, Fig. 50, $b, c$

inflammation of, in syphilis, Fig. 16

injury of, from iron splinter, Figs. $55, a, b, 57$

medullated nerve-fibers in, Fig. 6 opacity of, from blow, Figs. 51, 52

pigment of congenital defect of, Fig. 9

pigmentary degeneration of, Figs. $38,39,46, c$

pigmentation of, choroiditis and, Fig. 81

disseminated choroiditis and, Fig. 74

secondary, Fig. 46, $a$

in disseminated choroiditis, Fig. 43

senile, Fig. $72, b$

pigment-epithelium of, congenital defect of, Fig. 8, $b$

pulsation in, 82

reflexes on, 77

thrombosis of blood-ressels of, Fig. 33

Retinal arteries, syphilitic disease of, Fig. 36

bands and detachment, Fig. 60 from traumatism, Fig. 58, $b$

detachment, Figs. 59, 61

and bands, Fig. 60

funnel-shaped, Fig. 46, $b$

total, Fig. 46, $b$

with laceration, Fig. 62

separation, detection of, by transmitted light, 52

vessels, atrophy of, Fig. 58, I, $b$

Retinitis albuminurica, Figs. 26, 27, 29

of both eyes, Fig. 29

varicose nerve-fibers in, Fig. $26, b, c$

circinata, Fig. 64

diabetica, Fig. 30

hemorrhagic, Fig. 33, $a$ of pregnancy, hemorrhagic, Fig. 63

pigmentosa, Figs. 38, 39, 46, $c, d$ proliferans, Fig. 58, $b$

Retinoscopy, 48

Rules for observing eye-ground, 32
Sarcoma of choroid, Figs. 77, 78, 83

Schmidt-Rimbler method of measuring refraction, 45

Sclera, retina, and choroid, section through, Fig. 3

Scleral ring, 76

Sclerosis of choroidal vessels, Fig. 81

Senile macular disease, Fig. 47

Shadow-test, 48

in astigmatism, 52

Sickles, atrophic, Fig. 85

Skiascopy, 53

in astigmatism, 56

Solutio retinæ, Fig. 62

Staphyloma, true, Fig. 85

Strippling of eye-ground in macula lutea, 79

Superior temporal artery, obstruction of, Fig. 34

vein, thrombosis of, Fig. 33, a

Syphilis, congenital, eye-ground in, Figs. 41, 42

hereditary, eye-ground in, Fig. 40

inflammation of optic nerve in, Fig. 16

of retina in, Fig. 16

Syphilitic disease of retinal arteries, Figs. 36, 37

neuroretinitis, Fig. 37

Temporal artery, superior, obstruction of, Fig. 34

vein, superior, thrombosis of, Fig. 33, $a$

Thrombosis of central vein, Fig. $33, b$

of superior temporal artery, Fig. 34

vein, Fig. 33, a

of vena centralis retinæ, retina in, Fig. 50, $b, c$

Total hypermetropia, 30

Transmitted light, examination by, 47

Traumatic macular disease, Fig. 48

Tubercle, miliary, in choroid, Figs. $75,82, b$

Tubercular growth of choroid, Fig. $82, c$

Tuberculosis, acute miliary, of choroid, Fig. 75

chronic, of choroid, Fig. 76

Tumor, detection of, by transmitted light, 52 
Tumor, orbital, macula lutea in, |Vision, field of, size of, in direct Fig. 50, $a$

phantom, of choroid, Fig. 77

Türk on venous end-pulse, 86

VEINS, 80

vortex-, posterior, 82

Vena centralis retinæ, 80

thrombosis of, retina in, Fig. $50, b, c$

Venæ vorticosæ, posterior, Fig. 86

Venous pulse, 82, 83

Vision, field of, ophthalmoscopic, size of, 38

method, 38

in indirect method, 42

Vitreous, air-bubble in, Fig. 56

connective tissue in, Fig. 58, I, $b$

foreign body in, Fig. 53

detection of, by transmitted light, 50

hemorrhages into, Fig. 35

layer, hyaline bodies in, Figs. 72, $a, 82, a$

opacities in,. detection of, by transmitted light, 50

Vortex-veins, posterior, 82 
Gend

- $x$ of

$5 i 23=$

ris

,

(3) $x$

cots

Ex

$q^{2}+2=r=y$

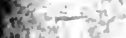

in

Q.

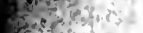

afsis

Sid sin

to

$x-3,2,1=$

Bgiti, 2

:

Eखं

es 


\section{UNIVERSITY OF CALIFORNIA LIBRARY}

\section{Los Angeles}

This book is DUE on the last date stamped below.

Form L9-Series 4939 


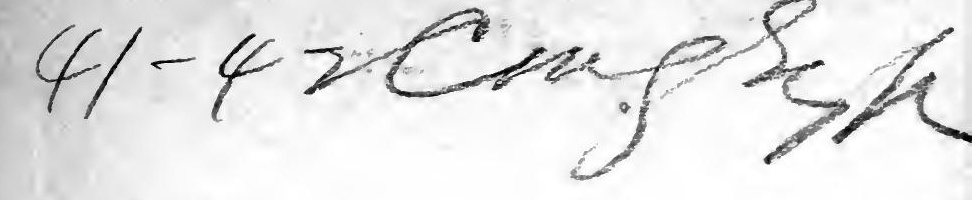


